$$
\begin{gathered}
\text { Universidade de São Paulo } \\
\text { Instituto de Física de São Carlos }
\end{gathered}
$$

Tiago Bueno de Moraes

\title{
Precessão Livre no Estado Estacionário com alternância de fase para RMN em alta e baixa resolução
}



Tiago Bueno de Moraes

\section{Precessão Livre no Estado Estacionário com alternância de fase para RMN em alta e baixa resolução}

Tese apresentada ao Programa de Pós-
Graduação em Física do Instituto de Física de
São Carlos da Universidade de São Paulo, para
obtenção do título de doutor em Ciências.

Área de Concentração: Física Aplicada

Orientador: Prof. Dr. Luiz Alberto Colnago

Versão Corrigida

(Versão original disponível na Unidade que aloja o Programa)

São Carlos 
AUTORIZO A REPRODUÇÃO E DIVULGAÇÃO TOTAL OU PARCIAL DESTE TRABALHO, POR QUALQÜER MEIO CONVENCIONAL OU ELETRÔNICO PARA FINS DE ESTUDO E PESQUISA, DESDE QUE CITADA A FONTE.

Ficha catalográfica revisada pelo Serviço de Biblioteca e Informação do IFSC, com os dados fornecidos pelo(a) autor(a)

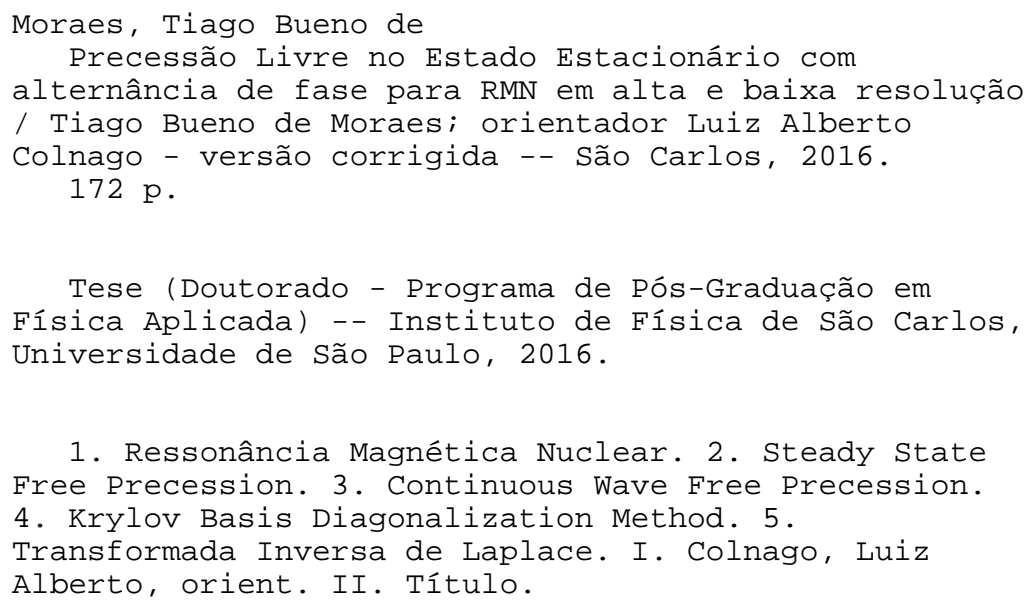


Dedico este trabalho aos meus pais Carlos e Teresinha pelo apoio incondicional em minhas aventuras científicas. 



\section{AGRADECIMENTOS}

Começo agradecendo meu guia no mundo incrível da Ressonância Magnética Nuclear, meu orientador Prof. Luiz Alberto Colnago. O convívio agradável e seu entusiamo nas dificuldades do dia-a-dia me fazem admirar sua trajetória e contribuições em sua carreira. Sem dúvida aprendi muito nesses anos.

Agradeço ao Prof. Cláudio J. Magon, Prof. Eduardo Azevêdo, Prof. Tiago Venâncio, ao Elton Montrazi e a Tatiana Moranetto pelas contribuições neste trabalho.

Ao Prof. Bernhard Bluemich e ao Ernesto Danieli, por compartilharem sua estrutura e conhecimento no mundo dos magnetos compactos na divertida cidade de Aachen.

Aos Professores do Instituto de Física de São Carlos, Alberto Tannús, Edson Vidoto, Pedro Donoso, Diogo Soares, Tito Bonagamba e Helmut Eckert, pelos conhecimentos transmitidos em aulas e conversas que influenciaram minha formação e o desenvolvimento desse trabalho.

Aos amigos da Embrapa Instrumentação - Maiara, Poliana, Luiza, Roberta, Gabi, Fabiana, Bruna, Esther, Cirlei, Isabel, Flávio, André, Djow, Rodrigo, Carlos, Diego, Paulo, Douglas, Giancarlo, Mario, Luiz - que através das múltiplas visões me mostraram o quão podemos aprender e crescer em um ambiente multi-disciplinar.

Aos amigos da República Bózons de Higgs, pela diversão e animadas discussões.

À Mariana que me aturou bem de perto nos momentos de alegrias e angústias, nessa eterna oscilação da vida na pesquisa científica.

À minha família, minha mãe e meu pai Teresinha e Carlos e irmãos Carla e José que sempre me apoiaram em tudo.

À Embrapa Instrumentação pelo apoio à realização deste projeto.

À FAPESP pelo apoio financeiro imprescindível à realização deste projeto.

Ao Instituto de Física de São Carlos - Universidade de São Paulo, que há uma década me recebeu tão bem e mostrou o caminho pelo qual se faz Ciência.

A todos que contribuíram de forma direta ou indireta para realização deste trabalho. 

Única testemunha do meu horizonte, comemorei sentado, quieto, com a boca cheia, a minha maior conquista: partir. Ainda que minha viagem durasse apenas um único e mísero dia. Parti para minha mais longa travessia, e, mesmo que ela só durasse esse único dia, eu havia escapado do maior perigo de uma viagem, da forma mais terrivel de naufrágio: não partir.

Amyr Klink 



\section{RESUMO}

MORAES, T.B. Precessão livre no estado estacionário com alternância de fase para RMN em alta e baixa resolução. 2016. 172 p. Tese de Doutorado - Instituto de Física de São Carlos, Universidade de São Paulo, São Carlos, 2016.

A aplicação de uma sequência de pulsos com tempo de repetição muito menor que os tempos de relaxação $T_{p}<<T_{2}, T_{1}$, faz com que a magnetização atinja um estado estacionário descrito por H.Y. Carr como Estado Estacionário em Precessão Livre, Steady State Free Precession (SSFP). Nessa condição, o sinal é composto pela complexa sobreposição das componentes FID e eco. Sequências tipo SSFP são utilizadas na aquisição rápida de sinais, resultando em uma boa razão sinal ruído $(\mathrm{s} / \mathrm{r}$ ) em curto intervalo de tempo, porém introduzem fortes anomalias de fase e amplitude devido a complexa interação das componentes que formam o estado estacionário. Neste trabalho, desenvolvemos sequências de pulsos tipo SSFP para RMN em alta e baixa resolução com alternância e incremento de fase. Em alta resolução desenvolvemos as sequências SSFPdx e SSFPdxdt com incremento de fase linear e quadrático respectivamente. Os resultados mostram que espectros de núcleos com baixa sensibilidade podem ser obtidos com mesma razão s/r em menor tempo experimental e as sequências desenvolvidas removem as anomalias espectrais. Em baixa resolução, os resultados mostram que a introdução de alternâncias de fase na Continuous Wave Free Precession (CWFP) possibilita a remoção da dependência da sequência com o offset de frequência e com o tempo entre pulsos. Além disso, mostramos que a sequência $C P-C W F P x-x$ com ângulo de refocalização pequeno $\left(5^{\circ}\right.$ a $\left.10^{\circ}\right)$ possibilita a estimativa rápida do tempos de relaxação longitudinal. Apresentamos também resultados dos estudos e desenvolvidos no estágio de pesquisa no exterior, onde as sequências de pulsos no estado estacionário - DECPMG e Split $180^{\circ}$ - foram estudas numericamente e implementadas nos sistemas magnéticos compactos: mini-Halbach e MOUSE-NMR. Por 
fim, são apresentados resultados com os métodos de processamento de dados Krylov Basis Diagonalization Method (KBDM) e a Transformada Inversa de Laplace aplicados na análise de sinais SSFP. Resultados mostram que KBDM é uma ferramenta útil no processamento de dados em alta e baixa resolução, tanto na obtenção de espectros como na determinação da distribuição dos tempos de relaxação.

PALAVRAS-CHAVE: Ressonância magnética nuclear. Steady state free precession. Continuous wave free precession. SSFP. CWFP. KBDM. Transformada inversa de Laplace 


\section{ABSTRACT}

MORAES, T.B. Steady state free precession with phase alternation for NMR in high and low resolution. 2016. 172 p. Tese de Doutorado - Instituto de Física de São Carlos, Universidade de São Paulo, São Carlos, 2016.

The application of a pulse sequence with repetition time much smaller than the relaxation times, $T_{p}<<T_{2}, T_{1}$, causes the magnetization to reach a steady state, described by $\mathrm{H}$. $\mathrm{Y}$. Carr as a Steady State Free Precession (SSFP). In this condition, the signal is composed of the complex overlapping of the FID and eco components. SSFP type sequences are used in fast acquisition of NMR signals, resulting in a good signal to noise ratio $(s / r)$ in a short time interval, however, they introduce phase and amplitude anomalies due to the complex interaction between the components of the steady state. In this work, we develop SSFP type pulse sequences for NMR in high and low resolution, with alternation and increment of phase. In high resolution, we develop SSFPdx and SSFPdxdt sequences, with linear and quadratic phase increment respectively. Results show that the low sensitivity nuclei spectra can be obtained with the same $s / r$ ratio in smaller experimental time, about an order of magnitude, and the developed sequences can remove the spectral anomalies. In low resolution, the results show that the introduction of a phase alternation in the Continuous Wave Free Precession (CWFP) allows the elimination of the dependence of the sequence with the offset frequency and the time between pulses. Besides, we show that the CP-CWFPx-x sequence with a small refocalization angle $\left(5^{\circ}\right.$ to $\left.10^{\circ}\right)$ allows the fast estimative of the longitudinal relaxation time in a single experiment. The results of the studies conducted during an international research internship are also presented. Steady state pulse sequences - DECPMG and Split $180^{\circ}-$ were studied and implemented in compact magnetic systems: mini-Halbach and MOUSE-NMR. 
Finally, the results of the application of the Krylov Basis Diagonalization Method (KBDM) and the Inverse Laplace Transform for the analysis of SSFP signals are presented. The results show that KBDM is a useful tool in data processing for low and high resolution, both for obtaining spectra and determining the relaxation times distribution.

KEYWORDS: Nuclear magnetic resonance. Steady state free precession. Continuous wave free precession. SSFP. CWFP. KBDM. Inverse Laplace transform 


\section{Lista de Figuras}

Figura 1.1 - Esquema da técnica de RMN pulsada com intervalos de tempos entre pulsos $T_{p}=T_{a q}+T_{r}$, onde $T_{a q}$ é o tempo de aquisição, $T_{r}$ tempo de reciclo, $\tau$ o tempo do pulso $\left(\tau<<T_{p}\right)$. Após cada pulso aplicado, um sinal transiente do retorno da magnetização ao equilíbrio é observado, denominado Free Induction Decay. . . . . . . . . . . . . . . . . . . . . . 37

Figura 2.1 - (a) Precessão do vetor magnetização ao redor do eixo da magnetização efetiva $B_{\text {ef }}$ durante a aplicação do pulso com amplitude $B_{1}$, fase $\pi / 2$ e frequência de offset $\gamma \Delta B_{0}$. (b) Precessão da componente durante a evolução livre. . . . . . . . . . . . . . . . . . . . . . . 48

Figura 2.2 - Sequência de pulsos com $T_{p}$ menor que os tempos de relaxação. A consequência de sequências de pulsos desse tipo é a estabilização de um estado estacionário da magnetização após um tempo transiente.

Figura 2.3 - Tempos imediatamente antes e após os pulsos de RF com duração $\tau$ e espaçados por $T_{p}$.

Figura 2.4 - Sinais teóricos do comportamento da magnetização em função do offset de frequência com $T_{p}=100 \mathrm{~ms}, T_{1}=5 \mathrm{~s}$ e $T_{2}=5 \mathrm{~s}$, em A) $\operatorname{com} \theta=$ $90^{\circ}$ e B) $\theta=30^{\circ}$. Na parte superior componentes real (preto), imaginária (vermelho) e módulo (azul) da magnetização; abaixo a fase dos sinal.

Figura 2.5 - Amplitude da magnetização no estado estacionário em função do ângulo dos pulsos $\theta$ e da razão $T_{1} / T_{2}$ em $A$ ) para isocromatas com ângulo de precessão $\Phi=\pi$ e em B) $\Phi=\pi / 6$. Os pontos $(\bullet)$ e a linha tracejada denotam as posição de $\theta_{o p}$ ótimo para cada razão $T_{1} / T_{2} \ldots$. . . . . . . 52 
Figura 2.6 - Após o início da aplicação de pulsos com ângulo $\theta$ a isocromata que precessiona com $\Phi=(2 n+1) \pi$ imediatamente antes $\left(M_{p}^{(a)}\right)$ e imediadamente depois $\left(M_{p}^{(d)}\right)$ da aplicação dos pulsos encontram-se no plano $y z$. Após aplicação de $n$ pulsos a magnetização antes e depois dos pulsos (componentes vermelhas) podem ser decompostas em termos da magnetização $M_{p}^{(a, d)}$ e $M_{w} \ldots \ldots \ldots \ldots$. . . . . . . . . . . 54

Figura 2.7 - Sinais simulados do perfil de excitação para três esquemas de alternância de fase em função do ângulo do pulso $\theta$ aplicado, com $T_{1}=T_{2}=1$ s e $T_{p}$ $=100$ ms. A linha (a) apresenta o perfil de excitação da sequência SSFP com fase constante para diversos valores do ângulo do pulso $\theta$. As linhas (b) e (c) apresentam os perfis para as sequências SSFP com alternância de fase $(x,-x)$ e $(x, x,-x,-x)$ respectivamente. . . . . . . . . . . . . 58

Figura 2.8 - Perfil de excitação para as sequências SSFP com incremento de fase, 0 , $\pi / 2, \pi$ e $3 \pi / 2$, denominadas SSFPdx. Vale ressaltar que a SSFP com incremento de fase $\pi$ resulta na mesma sequência com alternância $(x,-x)$ SSFPx-x . . . . . . . . . . . . . . . . . . 59

Figura 2.9 - Sinais simulados da SSFPdx com incremento linear de fase. O incremento de fase define as posições de máxima e mínima amplitude no eixo de frequência, assim, na situação com um pico na posição em ressonância, o sinal é suprimido para incremento 0 , aumenta para $\pi / 4$ e torna-se máximo $\left(M_{0} / 2\right)$ para incremento de fase $\pi$.

Figura 2.10 - Esquema de uma sequência de pulsos periódica geral, onde $\vec{R}_{A}\left(\hat{n}_{A}, \theta_{A}\right)$ é a rotação total dos pulsos iniciais aplicados, que geram a magnetização $\vec{M}\left(0^{+}\right)$e $R_{B}\left(\hat{n}_{B}, \theta\right)$ é a matriz de rotação total do intervalo periódico $T_{p}$ que resulta da composição das rotações de precessão livre e dos pulsos.

Figura 2.11 - Sinais simulados com $T_{1}=150 \mathrm{~ms}, T_{2}=50 \mathrm{~ms}, T_{2}^{*}=0,5 \mathrm{~ms}$ para os valores de tempo entre pulsos: A) $T_{p}=5 T_{1}$, B) $T_{p}=T_{2}$, C) $T_{p}=2,9 T_{2}^{*}$, D) and E) $T_{p}<T_{2}^{*}$. O offset de frequência é $8,333 \mathrm{KHz}(\mathrm{A}$ até $\mathrm{D})$ e $6,666 \mathrm{KHz}$ em E). 
Figura 2.12 - Magnitude dos sinais simulados para sequência de pulsos A) CWFP $[\pi / 2-$ $\left.T_{p}\right]_{n}$ e B) CP-CWFP $\pi-\frac{T_{p}}{2}\left[\pi / 2-T_{p}\right]_{n}$. Através dos parâmetros $M_{0}$, | $M_{s s} \mid$ e $T^{*}$ obtém-se em uma única aquisição os tempos de relaxação

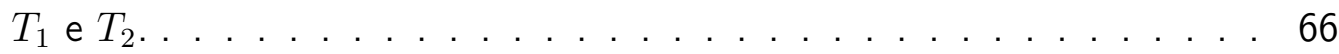

Figura 2.13 - Acima sequência de pulsos CPMG e abaixo Split $180^{\circ}$. Na Split $180^{\circ}$ dois pulsos de $90^{\circ}$ são espaçados por $\tau_{2}$. As fases associadas aos pulsos são $\phi_{0}$, $\phi_{1}$ e $\phi_{2}$, que para $\phi_{1}=\phi_{2}$ temos a Split $180^{\circ}$ Simétrica e para $\phi_{1}=-\phi_{2}$ Anti-simétrica. . . . . . . . . . . . . . . . . 6 67

Figura 2.14 - Sinais da sequência de pulsos Split $180^{\circ}$. Em A) observa-se o sinal CPMGlike (410) e o sinal que cresce até o estado estacionário (420). Em B) e C) são apresentados o perfil dos ecos formados no decaimento CPMG-like e no estado estacionário respectivamente. . . . . . . . . . . . . . . . 67

Figura 2.15 - Experimento bi-dimensional de correlação $T_{1}-T_{2}$. Em A) é apresentada a sequência de pulsos $\pi-\tau_{1}(i)-\frac{\pi}{2}-\frac{\tau}{2}-[\pi-\tau]_{n}$, onde $\tau_{1}(i)$ é uma lista de valores com $i$ experimentos na dimensão 1 . B) apresenta o perfil característico dos sinais adquiridos com tal sequência de pulsos e em C) após processamento do sinal com a Transformada Inversa de Laplace bidimensional o mapa de distribuição $T_{1}-T_{2}$ obtido. . . . . . . . . . 69

Figura 2.16 - Sequência de pulsos Driven-Equilibrium Carr-Purcell Meiboom-Gill DECPMG. 69

Figura 3.1 - Espectrômetro Avance III da Bruker de 14,1 T com frequência de ressonância de $600 \mathrm{MHz}$ para hidrogênio com sonda multinuclear de $5 \mathrm{~mm}$ de detecção inversa, localizado na Embrapa Instrumentação, São Carlos-SP.

Figura 3.2 - Espectrômetros de baixa resolução. Na esquerda acima SLK-200 e abaixo SLK-1399, ambos da SpinLock. Na direita acima Minispec mq20 da Bruker e abaixo Kea da Magritek. 
Figura 4.1 - Sinais CWFP simulados em A) sem alternância de fase e B) com alternância de $\pi$. Utilizando um incremento de fase $\varphi=\pi$ ( $\mathrm{x},-\mathrm{x}$ por exemplo) o perfil é deslocado de modo que os máximos de intensidade ficam presentes nos pontos de mínimo de intensidade da CWFP sem alternância de fase. Linhas contínuas é para $T_{p}=100 \mu \mathrm{s}$ e tracejada $60 \mu \mathrm{s}$. . . . . 79

Figura 4.2 - Sinais simulados das sequências de pulsos CWFP, CP-CWFP, CWFPx-x e CP-CWFPx-x com $T_{1}=100 \mathrm{~ms}, T_{2}=50 \mathrm{~ms}, T_{p}=300 \mu \mathrm{s}, T_{2}^{*}=20 \mu \mathrm{s} .80$

Figura 4.3 - A esquerda sinais CP-CWFP adquiridos de amostras de água dopada com cobre, em A) com concentração de 3,0 g/l, em B) com 0,47 g/l e em C) aquisição simultânea dos dois eppendorfs. A direita são apresentadas as respectivas Transformada inversa de laplace dos sinais, apresentando os tempos característicos encontrados.

Figura 4.4 - Variação do tempo característico $T^{*}$ da sequência CP-CWFPx-x em função do ângulo $\theta$ para soluções aquosas de $\mathrm{CuSO}_{4}$ e $\mathrm{MnSO}_{4}$, usando $T_{p}$ $=300 \mu \mathrm{s}$. As linhas sólidas foram calculadas pela equação 4.1.2. Os símbolos $(O)$ e $(\triangle)$ com preenchimento branco são os valores de $T_{1}$ obtidos com a sequência de pulsos IR

Figura 4.5 - Sinais CWFP- $T_{1}$ em A) simulados e em B) experimentais. Os índices i) até vi) são respectivamente para os ângulos $\theta$ de $90^{\circ}, 60^{\circ}, 45^{\circ}, 30^{\circ}, 20^{\circ}$, $10^{\circ}$ e $5^{\circ}$.

Figura 4.6 - Comparação da amplitude dos sinais CWFP- $T_{1}$ (cinza) e IR (pontos pretos), para água deionizada $T_{1}=3,9 \mathrm{~s}$. IR foi adquirido com 36 pontos em 9 minutos de experimento. CWFP- $T_{1}\left(10^{\circ}\right)$ adquirido em aquisição única em 27 segundos, com razão $s / r=17$. A figura interna apresenta um sinal FID da aquisição IR com $s / r=40$.

Figura 4.7 - Espectros ILT obtidos pelos sinais CPMG, CWFP- $T_{1}$ e IR de duas soluções $\mathrm{CuSO}_{4}$ e $\mathrm{MnSO}_{4}$ adquiridas simultaneamente em tubos concêntricos de 5 e 10 mm diâmetro. ILT dos sinais A) CPMG e G) Inversão Recuperação. Entre B) e F) ILT de sinais CP-CWFPx-x $\operatorname{com} \theta=90^{\circ}, 45^{\circ}, 30^{\circ}, 20^{\circ}$ e $10^{\circ} .88$ 
Figura 4.8 - Comparação da distribuição dos tempos de relaxação para amostras de A) carne com gordura e em B) apenas gordura. Os sinais IR foram obtidos com 36 pontos espaçados logaritmamente.

Figura 4.9 - Comparação da distribuição dos tempos de relaxação para amostras de A) Dendê e em B) Macadâmia. Os sinais IR foram obtidos com 36 e 144 pontos espaçados logaritmamente. Os sinais CWFP- $T_{1}$ foram adquiridos com $T_{p}=300 \mu$ s com 15 mil pontos na curva. Os processamento ILT foram realizados com parâmetro de regularização $\alpha=10$ e 400 pontos.

Figura 4.10 - Montagem do espectrômetro KEA com o magneto unilateral SingledSided NMR-MOUSE. . . . . . . . . . . . . . . . . . . . . . . . . . 91

Figura 4.11 - Montagem do espectrômetro KEA com o magneto mini-Halbach. . . . . . 91

Figura 4.12 - Sinais adquiridos com a Split $180^{\circ}$ para amostra de água dopada com cobre $T_{1} / T_{2}=1$. Em A) decaimento e crescimento dos ecos na Split $180^{\circ}$ no tempo. Em B) apresentamos o formato dos ecos na componente real da magnetização e em C) os ecos que crescem ao estado estacionário, usando $\tau_{1}=400 \mu \mathrm{s}, \tau_{2}=100 \mu \mathrm{s}, 512$ ecos e 512 médias no magneto NMR-MOUSE de $9 \mathrm{MHz}$.

Figura 4.13 - Simulação numérica da Split $180^{\circ}$ simétrica em função da inomogeneidade de campo $\vec{B}_{0} \hat{z}$ com largura de meia altura $F W H I=20 \mathrm{KHz}, 10 \mathrm{KHz}, 2$ $\mathrm{KHz}$ e $10 \mathrm{~Hz}$, usando $\tau_{1}=300 \mu \mathrm{s}, \tau_{2}=100 \mu \mathrm{s}, \phi_{0}=y, \phi_{1}=\phi_{2}=x$. Em A) e B) apresentamos o formato dos ecos do sinal CPMG-like e no estado estacionário respectivamente. C) apresenta o crescimento das amplitude dos ecos indo ao estado estacionário.

Figura 4.14 - Simulação numérica da Split $180^{\circ}$ anti-simétrica em função da inomogeneidade de campo $\vec{B}_{0} \hat{z}$ com largura de meia altura $F W H I=20 \mathrm{KHz}$, $10 \mathrm{KHz}, 2 \mathrm{KHz}$ e $10 \mathrm{~Hz}$, usando $\tau_{1}=300 \mu \mathrm{s}, \tau_{2}=100 \mu \mathrm{s}, \phi_{0}=y$, $\phi_{1}=-x$ e $\phi_{2}=x$. Em A) e B) apresentamos o formato dos ecos do sinal CPMG-like e no estado estacionário respectivamente. 
Figura 4.15 - Split $180^{\circ}$ simétrica para amostras de água dopada com cobre de diferentes valores de $T_{2}$ A) $3 \mathrm{~ms}$, B) $30 \mathrm{~ms}$ e C) $160 \mathrm{~ms}$. Note que a amplitude no estado estacionário é independente do valor de $T_{2}$ sendo a mesma amplitude uma vez que todos tem razão $T_{1} / T_{2}=1$.

Figura 4.16 - A linha superior apresenta sinais Split $180^{\circ}$ simétrica com $\tau_{1}=400 \mu \mathrm{s} \mathrm{e}$ A) $\tau_{2}=100$, B) $\tau_{2}=147$ e C) $\tau_{2}=200 \mu \mathrm{s}$. A linha inferior apresenta sinais de Split $180^{\circ}$ anti-simétrica com $\tau_{1}=400 \mu \mathrm{s} \mathrm{e} \mathrm{D)} \tau_{2}=100$, E) $\tau_{2}$ $=147$ e F) $\tau_{2}=200 \mu \mathrm{s}$. Experimentos foram realizados com amostra de água dopada com cobre com $T_{1}=T_{2}=30 \mathrm{~ms}, 512$ ecos e 512 médias. . 96

Figura 4.17 -Split $180^{\circ}$ anti-simétrica para diferentes razões $T_{1} / T_{2}$, para amostras de água dopada com cobre e três tipos de borracha. Em A) água dopada com cobre com $T_{1} / T_{2}=1$. B) borracha B. com $T_{1} / T_{2}=2,7, \mathrm{C}$ ) borracha $\mathrm{C}$. com $T_{1} / T_{2}=1,9$ e D) borracha P. com $T_{1} / T_{2}=6,7$. Sinais adquiridos com $\tau_{1}=400 \mu \mathrm{s}, \tau_{2}=200 \mu \mathrm{s}, 512$ ecos e 512 médias.

Figura 4.18 - Split $180^{\circ}$ anti-simétrica para dois tipos de queijo Gouda, com $\tau_{1}=400$ $\mu \mathrm{s}, \tau_{2}=200 \mu \mathrm{s}, 512$ ecos e 512 médias. A) com razão $T_{1} / T_{2}=4,3 \mathrm{e}$ B) e o segundo com $T_{1} / T_{2}=3,3$

Figura 4.19 - Simulação numérica da amplitude dos ecos no estado estacionário em função da razão $\tau_{2} / \tau_{1}$ e do offset de frequência para A) Split $180^{\circ}$ Simétrica $\phi_{1}=\phi_{2}$ e B) Split $180^{\circ}$ Anti-Simétrica $\phi_{1}=-\phi_{2}$. . . . . . . . . . . . 99

Figura 4.20 - Ecos no estado estacionário adquiridos no MOUSE-NMR no intervalo $\tau_{1}$ para as sequências A) Split $180^{\circ}$ simétrica e B) Split $180^{\circ}$ anti-simétrica. Foi utilizada água dopada com cobre $T_{1}=T_{2}=23 \mathrm{~ms}, \tau_{1}=400 \mu$ s e $\tau_{2}$

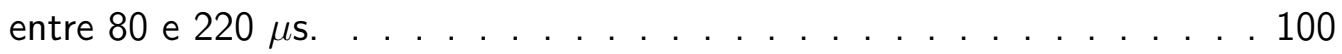

Figura 4.21 - Simulação numérica do perfil 'Butterfly' das sequências Split $180^{\circ} \mathrm{A}$ ) simétrica e B) anti-simétrica. Para $F W H M=20 \mathrm{KHz}, \tau_{1}=300 \mu$ s e $\tau_{2}$

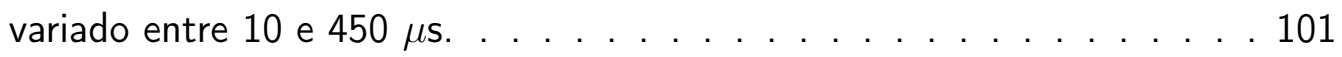


Figura 4.22 - Perfil 'Butterfly' experimental das sequências Split $180^{\circ}$ adquiridos no MOUSE-NMR para água dopada com cobre $T_{1}=T_{2}=23 \mathrm{~ms}$. Em A) Split $180^{\circ}$ simétrica e B) Split $180^{\circ}$ anti-simétrica. Com 512 ecos, 1024 médias e $\tau_{1}=400 \mu$ s e $\tau_{2}$ variado entre 40 e $400 \mu$ s em 144 passos. . . 102

Figura 4.23 - Comparação dos mapas de distribuição $T_{1}-T_{2}$ do conjunto água dopada e borracha, para as sequências de pulsos A) $T_{1}-T_{2}$ bi-dimensional com tempo total de aquisição 2,5 minutos e B) DECPMG unidimensional, com tempo de aquisição de 10 segundos.

Figura 4.24 - Comparação dos mapas de distribuição $T_{1}-T_{2}$ do conjunto queijo D. e leite, para as sequências de pulsos A) $T_{1}-T_{2}$ bi-dimensional e B) DECPMG unidimensional.

Figura 4.25 - Comparação dos mapas de distribuição $T_{1}-T_{2}$ do conjunto queijo $\mathrm{M}$. e leite, para as sequências de pulsos A) $T_{1}-T_{2}$ bi-dimensional e B) DECPMG unidimensional. 104

Figura 4.26 - Sequência de pulsos Driven-Equilibrium modificada, onde o intervalo $\tau_{2}$ passa a ser uma variável $\tau_{2}(i)$ com $i$ componentes. Repetindo esse bloco de pulsos $n$ vezes a magnetização atinge um estado estacionário dependente das razões $\tau_{1} / \tau_{2}$ e $T_{1} / T_{2} \ldots \ldots \ldots$. . . . . . . . . . . . . . . . . . . .

Figura 4.27 -Em A) sinais simulados da sequência Driven-Equilibrium modificada com a amplitude em função da lista $\tau_{2}(i) / \tau_{1}$, para $T_{1} / T_{2}=1,1,3$ e 2 . B) apresenta sinal experimental apresentando o comportamento da magnetização na passagem de um estado estacionário ao próximo, onde $i$ denota a transição da lista $\tau_{2}(i) / \tau_{1} \ldots \ldots$. . . . . . . . . . . . . . . . . . . .

Figura 4.28 - Comparação das razões $T_{1} / T_{2}$ obtidas com as sequências convencionais cpmg e inversão recuperação com a Driven-Equilibrium modificada. Em A) para polímeros pec $2 k$, pec4k, pec6k e pec8k em triplicata e em B) para óleos comestíveis Azeite de Oliva, Colza, Gergelim e Linhaça em decuplicata. 
Figura 5.1 - Sinais de ${ }^{1} \mathrm{H}\left(\mathrm{H}_{2} \mathrm{O} / \mathrm{D}_{2} \mathrm{O}\right)$ para as sequências A) Pulso simples (zg), B) Pulsos simples truncada em $30 \mathrm{~ms}$, com tempo de reciclo longo e C) SSFP com $T_{a q}=29.7, d_{1}=0.3 \mathrm{~ms}$, todos com pulso de $\theta=90^{\circ}$ e 4 médias. . . . . . . . . . . . . . . . . . . 108

Figura 5.2 - A) Apresenta a sequência de FIDs adquiridos na SSFP evidenciando o comportamento da magnetização até estabelecimento do Estado Estacionário após 2 segundos. Em B) e C) temos a ampliação das regiões do início e final, a regiões sem sinal são as posições de aplicação de pulsos de RF. Pulsos de RF tem cerca de $10 \mu$ s de duração e a figura não está em escala. . . . . . . . . . . . . . . . . . . . . . . . 109

Figura 5.3 - FIDs SSFP para quatro valores de offset de frequência: A) 2888, B) 2904,66, C) 2954,64 e D) 2938 Hz. Os sinais A) e C) em offset com mínimo de amplitude, onde a componente eco é evidente, e os sinais em B) e D) em posição de máximo de amplitude.

Figura 5.4 - A) Componentes FID e eco resultantes da deconvolução do FID-SSFP pelo Método da Diagonalização Filtrada. B) FID original e a linha residual do fitting com o dado experimental.

Figura 5.5 - Sinal SSFP-stopped adquirida da amostra de $\mathrm{H}_{2} \mathrm{O} / \mathrm{D}_{2} \mathrm{O}$ com $T_{p}=100$ ms e fase constante. Em B) uma ampliação dos três primeiros intervalos entre 0 e $0,3 \mathrm{~s}$.

Figura 5.6 - A) e B) apresenta os sinal SSFP adquiridos em offset de frequência 2887 $\mathrm{Hz}$ e $2892 \mathrm{~Hz}$ respectivamente, sendo um a situação de máxima amplitude de sinal e a outro mínima. Em C) e D) apresentamos os sinais adquiridos com a sequência SSFPx-x, onde o offset para máximo de sinal é $2892 \mathrm{~Hz}$ e mínimo em $2887 \mathrm{~Hz}$ 
Figura 5.7 - Perfil de excitação de ${ }^{1} \mathrm{H}$ para $\mathrm{H}_{2} \mathrm{O} / \mathrm{D}_{2} \mathrm{O}$ (à esquerda) e para $\mathrm{H}_{2} \mathrm{O}$ com $\mathrm{C}_{3} \mathrm{H}_{6} \mathrm{O} / \mathrm{D}_{2} \mathrm{O}$ (à direita) para sequências SSFP com diferentes valores de $\theta$ A) $90^{\circ}$, B) $60^{\circ}$, C) $30^{\circ}$ e D) $5^{\circ}$, com $T_{p}=100 \mathrm{~ms}$ e 4 médias. $O$ contorno vermelho destaca a modulação da amplitude do pico da acetona e o contorno preto da água. . . . . . . . . . . . . . . . . . 113

Figura 5.8 - Perfil de excitação $\mathrm{H}_{2} \mathrm{O} / \mathrm{D}_{2} \mathrm{O}$ para sequências SSFPdx para diferentes valores de incremento de fase $\delta \varphi$, A) 0, B) $\pi / 4$, C) $\pi / 2$, D) $3 \pi / 4$, E) $\pi$, F) $5 \pi / 4$, G) $3 \pi / 2$, H) $7 \pi / 4$.

Figura 5.9 - Múltiplas aquisições SSFPdx-n2, SSFPdx-n4 e SSFPdx-n8, utilizam-se respectivamente de 2, 4 e 8 blocos de aquisição SSFPdx, onde cada bloco possui um valor de incremento de fase $\delta \varphi$ de $0, \pi / 4, \pi / 2,3 \pi / 4$,

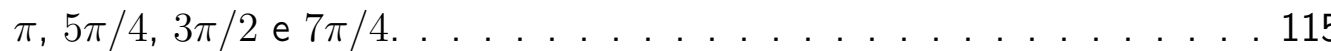

Figura 5.10 - A direita perfil de excitação $\mathrm{H}_{2} \mathrm{O} / \mathrm{D}_{2} \mathrm{O}$ para sequências A) SSFPdx-n2, B) SSFPdx-n4 e C) SSFPdx-n8. A esquerda perfil de excitação água e acetona para sequências $A^{\prime}$ ) SSFPdx-n2, B') SSFPdx-n4 e C') SSFPdx$\mathrm{n} 8$, todos adquiridos com $T_{p}=100 \mathrm{~ms}, \theta=90^{\circ}$ e 8 médias.

Figura 5.11 - Sinais simulados da SSFPdxdt com incremento não-linear de fase. A magnetização no estado SSFPdxdt apresenta modulação temporal de acordo com o fator $A$ do incremento de fase utilizado onde apresentamos desde $A=\pi / 16$ até $\pi / 2048$. Em A) vemos que para $A$ menor que $\pi / 128$ a amplitude máxima do sinal cresce conforme menor o valor de $A$. Em B) vemos que após um valor pequeno o suficiente de $A$ a amplitude máxima dos sinais fica em $M_{0} / 2$. 
Figura 5.12 - Sinais de $\mathrm{H}_{2} \mathrm{O} / \mathrm{D}_{2} \mathrm{O}$ apresentando o comportamento da magnetização quando submetida a sequência A) SSFP sem incremento de fase e na posição de mínima intensidade, B) SSFP sem incremento de fase e na posição de máxima intensidade, C) SSFPdxdt com $A=\pi / 32$, D) $A=$ $\pi / 64$, E) $\mathrm{A}=\pi / 128$ e F) $\mathrm{A}=\pi / 256$. Nas SSFPdxdt a amplitude no estado estacionário é modulada no tempo, alternando entre máximo e mínimo de intensidade ao longo do tempo, além disso a amplitude máxima do sinal é maior quanto menor valor de $\mathrm{A}$.

Figura 5.13 - Em A) temos a comparação entre a SSFP sem incremento de fase (preto) em seu máximo de intensidade no estado estacionário, junto com o sinal SSFPdxdt usando $\mathrm{A}=\pi / 256$ (vermelho). Temos que a magnitude do sinal SSFPdxdt possui caráter periódico, correspondente ao deslocamento do perfil de excitação no eixo das frequências, como apresentado em B). A seta vermelha em B) representa um pico do espectro que em cada scan apresenta uma amplitude modulada correspondente as posições 1 à 5 de A). . . . . . . . . . . . . . . . . . . . . . . 119

Figura 5.14 - Comparação entre perfil de excitação das sequências SSFPdxdt com A sendo A) $\pi / 256$, B) $\pi / 128$, C) $\pi / 64$, D) $\pi / 32$, E) $\pi / 16$ e com a SSFP sem incremento de fase em F).

Figura 5.15 - Comparação entre os espectros de ${ }^{13} C$ Sacarose adquiridos com as sequências A) padrão (zgpg30), B) SSFP, C) SSFPdx-n2, D) SSFPdx-n4 e E) SSFPdx-n8, todos com 8192 médias.

Figura 5.16 - A esquerda comparação dos espectros de ${ }^{13} C$ da sacarose adquiridos com as sequências A) padrão (zgpg30), B) SSFP sem alternância de fase e adquiridos com SSFPdxdt com incremento de fase em C) pi/256, D) $\pi / 64$ e E) $\pi / 16$, com 256 médias, $T_{p}=200$ ms. A direita intensidade do pico em 104 ppm em função do incremento de fase na SSFPdxdt. . 124 
Figura 5.17 -Espectros de ${ }^{13} C$ do etil bezeno em clorofórmio com aquisição pela sequência A) zgig, B) SSFPdxdt $\pi / 256$, C) SSFPdxdt-n8. A direita espectros adquiridos com a SSFPdxdt $\pi / 256$ com pulsos de refocalização $\left.\left.\operatorname{de} A^{\prime}\right) \theta=30^{\circ}, B^{\prime}\right) 10^{\circ}$ e $\left.C^{\prime}\right) 5^{\circ}$.

Figura 5.18 - Espectros de ${ }^{15} \mathrm{~N}$ de anilina e trietilamina para as sequências A) padrão (zg30) com 3h e 30 minutos de aquisição, B) SSFPdxdt $\pi / 256$ com 30 minutos de aquisição, C) padrão com 15 minutos aquisição e D) SSFPdxdt $\pi / 256$ com 15 minutos de aquisição. O espectro C) foi ampliado 4 vezes para melhor visualização.

Figura 5.19 - Espectros de ${ }^{15} \mathrm{~N}$ da Formamida $\left(\mathrm{CH}_{3} \mathrm{NO}\right)$ para as sequências: A) Padrão (zg), B) SSFP, C) SSFPdx-n8 e D) SSFPdxdt com $\pi / 256$ e $T_{p}=$ $100 \mathrm{~ms}$. E) e F) apresenta o sinal SSFPdxdt adquirido com $T_{p}=200 \mathrm{~ms}$, processado respectivamente com a TF e KBDM. G) e H) sinal SSFPdxdt adquirido com $T_{p}=400 \mathrm{~ms}$, processado respectivamente com a TF e KBDM.

Figura 6.1 - Componente real do FID obtido com a sequência de pulsos A) convencional (zgpg30) e B) SSFP com 100 ms de aquisição. Em C) apresentamos a componente eco retirada do sinal SSFP através do processamento com o KBDM.

Figura 6.2 - Componente absortiva do espectro de Fourier de ${ }^{13} \mathrm{C}$ da Brucina/DMSO$d_{6}$ adquirida com a sequência de pulsos convencional (zgpg30) e tempo total de experimento de 90 minutos.

Figura 6.3 - Espectros de ${ }^{13} C$ Brucina adquiridos com SSFP com tempos de aquisição de a) $100 \mathrm{~ms}$, b) $50 \mathrm{~ms}$ e c) $25 \mathrm{~ms}$. Em A) apresentamos o espectro gerado pelo processamento com a Transformada de Fourier com função Traf expandindo duas regiões do espectro. Em B) comparamos os espectros gerados pelo processamento do mesmo sinal SSFP com o método KBDM. 134

Figura 6.4 - Sinais ${ }^{13} C$ SSFP sem alternância de fase da o solvente DMSO- $d_{6}$, processados com a Transformada de Fourier e com o KBDM. 
Figura 6.5 - Regiões do espectros de ${ }^{13} C$ SSFPdx com 8 blocos de aquisição da amostra Brucina/DMSO- $d_{6}$, processados com a Transformada de Fourier e com

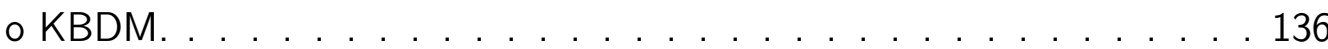

Figura 6.6 - Regiões do espectros de ${ }^{13} C$ SSFPdxdt com incremento $\pi / 256$ da amostra Brucina/DMSO- $d_{6}$ com $T_{p}=200 \mathrm{~ms}$, processados com a Transformada de Fourier e com o KBDM. . . . . . . . . . . . . . . . . 137

Figura 6.7 - Um sinal composto pela soma de decaimentos exponenciais é um problema matemático mal-posto, pois mais de uma solução é possível. A Transformada Inversa de Laplace em conjunto com métodos de regularização, como o SVD e Tikhonov, forçam uma solução de acordo com um parâmetro $\alpha$ escolhido, resultando na distribuição de tempos como em B). 138

Figura 6.8 - Esquema do método KBDM para obtenção de distribuição de tempos de relaxação. Através da introdução do ruído branco (PNA) no sinal CPMG, é realizada média sobre os cálculos do KBDM, resultando na distribuição das possíveis soluções do sistema, ou seja, num histograma da distribuição dos tempos de relaxação $T_{2} \ldots \ldots$. . . . . . . . . . . . . . . . . . 144

Figura 6.9 - Visualização da lista de parâmetros obtida na análise espectral com o KBDM do sinal CPMG da cerâmica de alumina. Em A) temos a visualização completa da faixa de frequência e em B) a região próximo de zero expandida. Uma vez que estamos parametrizando um sinal composto por uma soma de exponenciais com frequência nula, apenas as componentes selecionadas em B) já retornam um ótimo ajuste do sinal experimental. Os demais pontos são gerados no processo de fitting devido o ruído presente no sinal. O processamento KBDM foi realizado com PNA $2 \%$ e 100 médias computacionais. . . . . . . . . . . . . . . . . . 145 
Figura 6.10 - Comparação da distribuição de $T_{2}$ através do método do KBDM (esquerda) e do método da Inversão de Laplace (direita) em função do número de médias experimentais do sinal CPMG das cerâmicas de alumina, com A) 2, B) 8, C) 32 e D) 128 médias. As distribuições por KBDM foram calculadas com 100 médias computacionais e PNA de 2\%. As distribuições de Laplace foram calculadas com $\alpha=10$ e 100 pontos. . . 146

Figura 6.11 - Comparação das distribuições $T_{2}$ obtidas pelo KBDM, em função do número de médias computacionais realizada, A) 4, B) 16, C) 64 e D) 256. Todas as distribuições foram calculadas com PNA $=2 \%$ e com o a sinal experimental obtido com 32 scans da cerâmica de alumina.

Figura 6.12 - Comparação da distribuição de $T_{2}$ pelo método do KBDM (esquerda) e Inversão de Laplace (direita) calculada em função do parâmetro de regularização (PNA) no caso do FDM com 0, 2\%, 10\%, 15\% e E) 150\%. Para a Inversão de Laplace foi calculado em função do parâmetro de regularização $\left.\alpha A^{\prime}\right)$ 0,001, B') 0,1, C') 1, D') 100 e E') 10.000, todos com 200 pontos.

Figura 6.13 - A) Comparação da distribuição de $T_{2}$ pelo método do KBDM (vermelho) e Inversão de Laplace (preto) para os sinais adquiridos duas a duas, das soluções aquosas de $\mathrm{CuSO}_{4}$, de baixo pra cima $(\mathrm{S} 1+\mathrm{S} 12),(\mathrm{S} 2+\mathrm{S} 12)$ até $(\mathrm{S} 11+\mathrm{S} 12)$. As Inversões da Laplace foram realizadas com $\alpha=0,1$. Em B) é apresentada em azul os valores de $T_{2}$ de cada solução $S_{9}$ até $S_{12}$, comparados com os valores obtidos pelo KBDM (vermelho) e pela Inversão de Laplace (preto) $\operatorname{com} \alpha=0,0001$. Com $\alpha$ reduzido é possível a presença de dois picos, porém com pouca precisão.

Figura 6.14 - Distribuição de tempos $T_{2}$ pela ILT e KBDM para A) amendoim umidecido, B) Óleo lubrificante e C) Rochas porosas Sillurian, Indiana e Combfield. Em vermelho sinais gerados pelo KBDM, azul Laplace com $\alpha$ maior e preto $\alpha$ menor. 
Figura A.1 - Esquema da SSFP em alta resolução para determinação rápida dos tempos de relaxação $T_{1}$ e $T_{2}$. Em A) temos a aquisição de todo o comportamento da magnetização até a estabilização do estado estacionário. B) apresenta as Trans. de Fourier de cada período da aquisição, onde através dos tempos de decaimento dos picos e das amplitudes $M_{0}$ e $M_{s s}$ é possível se determinar os tempos de relaxação.

Figura A.2 - A) apresenta o sinal adquirido com a sequência Inversão Recuperação para o pico da água e B) para o pico da acetona. C) e D) apresentam respectivamente os sinais adquiridos com a sequência CPMG para o pico da água e da acetona no espectrômetro Bruker 600 MHz. . . . . . . . . 169

Figura A.3 - Decaimento do sinal CWFPx-x em alta resolução $(600 \mathrm{MHz})$ para amostra de $\mathrm{H}_{2} \mathrm{O}$ e acetona até estabilização no estado estacionário do pico da A) água e em B) acetona. . . . . . . . . . . . . . . . . . . . . . . . . . . 169

Figura B.4 - Código implementado na plataforma do Matlab para execução da Transformada Inversa de Laplace. . . . . . . . . . . . . . . . . . . . . . . . . 170

Figura C.5 - Sequência de pulsos SSFPdx-n4 com incremento linear de fase, com quatro blocos de aquisição.

Figura C.6 - Sequência de pulsos SSFPdxdt com incremento não linear de fase, utilizando $A=\pi / 64$. 


\section{Lista de Tabelas}

Tabela 1.1 - Tabela apresenta alguns fatores que influenciam no tempo total de aquisição em RMN. O tempo experimental é estimado de acordo com a abundância natural, sensibilidade e influenciado pelo tempo de relaxação dos núcleos.

Tabela 4.1 - Comparação dos tempos de relaxação (ms) obtidos com as sequências de pulsos IR e CWFP- $T_{1}$ para amostras com diferentes razões $T_{1} / T_{2}$. Os experimentos foram realizados em triplicata com $T_{p}=300 \mu$ s na CWFP$T_{1}$ e listas com $36 \tau$ s na aquisição IR.

Tabela 4.2 - Esquema de ciclo de fases para Split $180^{\circ}$ simétrica e anti-simétrica para aquisição do sinal steady-state ou cpmg-like. De cima pra baixo fase dos pulsos $\phi_{0}, \phi_{1}, \phi_{2}$ e receptor.

Tabela 4.3 - Tempos de relaxação das amostras com as sequências de pulsos CPMG e Inversão Recuperação.

Tabela 5.1 - Esquema de fases da SSFPdxdt para A igual a $\pi / 2, \pi / 4, \pi / 8, \pi / 16$, $\pi / 32, \pi / 64, \pi / 128$ e $\pi / 256$.

Tabela 5.2 - Parâmetros utilizados nas sequências de pulso padrão (zgpg30), SSFP, SSFPdx-n2, SSFPdx-n4 e SSFPdx-n8.

Tabela 5.3 - Tempos de relaxação $T_{1}$ e $T_{2}$ para cada pico do espectro de ${ }^{13} \mathrm{C}$ da sacarose, obtidos pelas técnicas cpmg e t1irpg.

Tabela 6.1 - Tempos de relaxação $T_{2}$ de cada solução. 148 
Tabela A.1 - Comparação tempos de relaxação obtidas com sequências CPMG, Inv. Rec. e CWFP-HR. . . . . . . . . . . . . . . . . . . . . . . . 169 


\section{Sumário}

1 Introdução 33

1.1 Ressonância Magnética Nuclear . . . . . . . . . . . . . . . . . . . . . 33

1.2 Precessão Livre no Estado Estacionário . . . . . . . . . . . . . . . . . 38

1.3 Motivação e objetivos . . . . . . . . . . . . . . . . . . . . . . . . . . 40

2 Magnetização no Estado Estacionário 45

2.1 Evolução da magnetização em campos magnéticos inomogêneos $\vec{B}_{0}(\vec{r})$

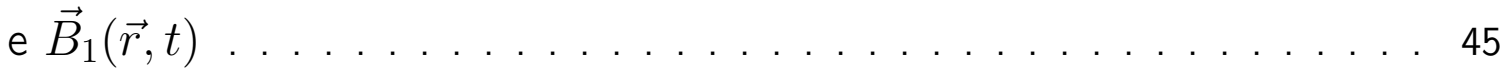

2.2 Amplitude da magnetização no Estado Estacionário . . . . . . . . . . . . 49

2.3 Transiente ao Estado Estacionário . . . . . . . . . . . . . . . . . . 53

2.4 Alternância de fase no Estado Estacionário . . . . . . . . . . . . . . . . 56

2.4.1 Perfil de Excitação . . . . . . . . . . . . . . . . . . . . . . . 57

2.5 Sinais assintóticos em estruturas periódicas . . . . . . . . . . . . 61

2.5.1 Continuous Wave Free Precession (CWFP) . . . . . . . . . 62

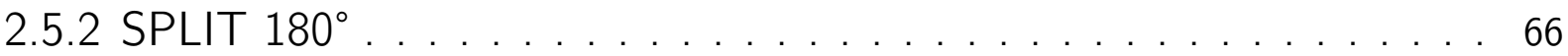

2.5.3 Driven-Equilibrium CPMG . . . . . . . . . . . . . . . 68

3 Materiais e Métodos $\quad 71$

3.1 Espectrômetros e magnetos . . . . . . . . . . . . . . . . . . . . 71 
3.1 .1 Alta resolução . . . . . . . . . . . . . . . . . . . . . . . . 71

3.1 .2 Baixa resolução . . . . . . . . . . . . . . . . . . . . . 71

3.2 Preparo de amostras . . . . . . . . . . . . . . 73

3.2 .1 Seção 5 - SSFP . . . . . . . . . . . . . . . . . . . . 73

3.2 .2 Seção 6.2 - KBDM . . . . . . . . . . . . . . . . . . . 73

3.3 Programação das sequências de pulsos e simulações numéricas . . . . 74

3.4 Processamento de dados . . . . . . . . . . . . . . . . . . . 75

4 Estado Estacionário em Baixa Resolução 77

4.1 Continuous Wave Free Precession . . . . . . . . . . . . . . 78

4.1.1 CWFP com alternância de fase . . . . . . . . . . . . . . 78

4.1.2 CWFP para análise multi-exponencial . . . . . . . . . . . . . 81

4.2 Estado Estacionário em magnetos compactos . . . . . . . . . . . 90

4.2.1 Split $180^{\circ}$ e Butterfly Pathways . . . . . . . . . . . . . . . 92

4.2 .2 DECPMG . . . . . . . . . . . . . . . . . . . . 101

4.2 .3 Driven-Equilibrium modificada . . . . . . . . . . . . . . . . 104

5 Estado Estacionário em Alta Resolução 107

5.1 Caracterização experimental da SSFP . . . . . . . . . . . . . . . 107

5.2 SSFPdx com incremento linear de fase . . . . . . . . . . . 113

5.3 SSFPdxdt com incremento não-linear de fase . . . . . . . . . . . . 116

5.4 Núcleos com baixa sensibilidade . . . . . . . . . . . . . . . . . . . 121

5.4 .1 Carbono-13 . . . . . . . . . . . . . . . . . . . . . 122

5.4 .2 Nitrogênio-15 . . . . . . . . . . . . . . . . . . . . 127 
5.5 Resolução, tempo e razão sinal/ruído . . . . . . . . . . . . . . . . . 129

6 Diagonalização Filtrada e Inversa de Laplace 131

6.1 KBDM para supressão das anomalias na SSFP . . . . . . . . . . . 132

6.2 Distribuição de tempos de relaxação . . . . . . . . . . . . . . . . 137

6.2 .1 Transformada Inversa de Laplace . . . . . . . . . . . . . . . . 139

6.2.2 Análise exponencial via KBDM . . . . . . . . . . . . . . . . . . 141

7 Conclusão 153

8 Perspectivas 155

$\begin{array}{ll}\text { Referências } & 157\end{array}$

$\begin{array}{ll}\text { Apêndices } & 167\end{array}$

Apêndice A: SSFP para determinação dos tempos de relaxação . . . . . . . 167

Apêndice B: Pacote Matlab: Transformada Inversa de Laplace . . . . . . . 170

Apêndice C: Sequências de pulsos SSFP . . . . . . . . . . . . . . . . 171 



\section{Capítulo 1}

\section{Introdução}

\subsection{Ressonância Magnética Nuclear}

Experimentos de Ressonância Magnética Nuclear (RMN) detectam a interação de spins nucleares - por exemplo, núcleos de ${ }^{1} H,{ }^{13} C,{ }^{15} N$, etc - com a radiação eletromagnética, na faixa de ondas de radio $(\mathrm{MHz})$, quando imersos em um campo magnético.

No início da década de 1920 Wolfgang Pauli propôs, similar ao spin do elétron, a existência de momento magnético nos núcleos de alguns átomos, ao tentar explicar a estrutura hiperfina em linhas espectrais. A hipótese do spin nuclear foi observada inicialmente por Rabi em feixes de partículas em altas energias ${ }^{1}$ até que em 1945 dois grupos de pesquisa independentes conseguiram observar os efeitos dos spins nucleares em amostras líquidas e sólidas - matéria condensada - dando início a base moderna da espectroscopia de Ressonância Magnética Nuclear.

O grupo de Harvard (Purcell, Torrey, Pound) ${ }^{2}$ entendia o experimento de RMN em termos das transições entre os estados quânticos, enquanto o grupo de Stanford (Bloch, Hansen, Packard) ${ }^{3,4}$ visualizava esses experimentos em termos da magnetização macroscópica dos momentos magnéticos nucleares na presença de um campo magnético externo. Posteriormente, ficou evidente que ambas as explicações eram complementares e por essa descoberta Bloch e Purcell receberam o Prêmio Nobel de Física em 1952.

Logo no início da década de 1950, vários trabalhos evidenciavam de maneira inesperada existência de múltiplas ressonâncias, tendo correta interpretação no trabalho de M.E. Packard 
et al. ao identificar os três picos de frequências distintos no ${ }^{1} \mathrm{H}$ com os grupos químicos do etanol $\left(\mathrm{CH}_{3} \mathrm{CH}_{2} \mathrm{OH}\right) \cdot{ }^{5}$ Essa observação foi então compreendida como resultado do efeito do deslocamento da frequência de ressonância causado pelas nuvens eletrônicas em cada região da molécula, efeito denominado Deslocamento Químico. Percebeu-se assim que através do deslocamento químico é possível inferir e identificar estruturas químicas de praticamente qualquer molécula orgânica ou inorgânica que apresente sinais de RMN, como por exemplo moléculas com núcleos de ${ }^{1} \mathrm{H},{ }^{13} \mathrm{C},{ }^{15} \mathrm{~N}$ e ${ }^{19} \mathrm{~F}$.

As ricas informações obtidas com a técnica de RMN fez surgir um grande interesse pela comunidade cientifica e os primeiros entusiastas enfrentavam o desafio de construir seu próprio espectrômetro. Logo as primeiras companhias iniciaram o desafio de tornar aqueles espectrômetros complexos em um produto comercial e acessível a pesquisa para as diversas áreas do conhecimento, sendo a Varian protagonista fundamental dessa transição que levou mais de uma década, resultando no primeiro grande sucesso com o espectrômetro 'A-60' introduzido em 1961. ${ }^{6}$

Sabia-se na época que um espectro de frequências da amostra analisada poderia ser obtido a partir dos experimentos de RMN, porém o grande desafio era como executar isso tecnicamente, com as limitações eletrônicas e computacionais da época.* Médias dos sinais adquiridos em tempo real foram disponíveis apenas na segunda metade da década de 60 junto os métodos numéricos da Transformada de Fourier Discreta (DFT), com os primeiros trabalhos de FTNMR desenvolvidos por R. Ernest e W. Anderson. ${ }^{7}$

Tais desenvolvimentos culminaram em uma revolução na sensibilidade da RMN, começando em 1966 com a técnica proposta de R. Ernest e W. Anderson ${ }^{11}$ de utilizar pulsos curtos de RF para excitar o sistema de spins, seguido da aquisição de seu sinal transiente e processamento com Transformada de Fourier. Apesar de ter demorado alguns anos para essa técnica ser viável em espectrômetros comerciais o método é a base das técnicas espectroscópicas de RMN de alta resolução nos dias atuais. ${ }^{\dagger}$

\footnotetext{
* A Transformada de Fourier Discreta foi desenvolvida no início da década 60 e demorava cerca de 20 minutos pra analisar um espectro. ${ }^{7}$ A Fast Fourier Transform (FFT) surgiu em $1965^{8}$ e a Transformada Inversa de Laplace numérica (CONTIN) ${ }^{9,10}$ no início da década de 80.

$\dagger$ Vale comentar o fato de que essa proposta estava tão a frente de seu tempo que o artigo foi rejeitado duas vezes pela J. Chem. Phys. e então aceito na Rev. Sci. Instr. ${ }^{12,13}$
} 
Na próxima seção introduzimos os elementos básicos de um experimento de RMN pulsado, com definições úteis para acompanhar a descrição da magnetização no estado de precessão livre no estado estacionário do capítulo 2.

\section{Conceitos básicos}

A espectroscopia de RMN é baseada na observação dos níveis de energia descritos pelo Efeito Zeeman dos spins nucleares imersos em um campo magnético. O spin nuclear é um efeito puramente quântico e uma descrição mais completa do fenômeno pode ser encontrada em diversos livros textos. ${ }^{14-18}$

A diferença de energia dos níveis degenerados para spins $1 / 2$ imersos em um campo externo $\vec{B}_{0} \hat{z}$ é dada por $\Delta E_{\alpha \beta}=\gamma \hbar B_{0}$, entre os níveis de menor $\alpha$ (spin up) e maior energia $\beta$ (spin down), onde $\hbar$ é a constante de Planck e $\gamma$ é a constante magnetogírica do núcleo. A distribuição de população de spins entre os níveis de energia $\alpha$ e $\beta$ é dada pela distribuição de Boltzmann

$$
\frac{N_{\beta}}{N_{\alpha}}=e^{-\frac{\Delta E}{k T}}=e^{-\frac{\gamma \hbar B_{0}}{k T}}
$$

onde $k$ é a constante de Boltzmann e T a temperatura.

Uma vez que a diferença de energia entre os níveis é muito pequena, a diferença de população também é pequena. Por exemplo, em um campo magnético de 1 Tesla, a razão de população para um núcleo de hidrogênio a temperatura ambiente de $300 \mathrm{~K}$ é $N_{\alpha}=$ $1,000006811 N_{\beta}$. Essa pequena diferença de população é um dos fatores da intrínseca baixa sensibilidade da espectroscopia de RMN.

Muitos fenômenos na RMN com spins $1 / 2$ não interagentes podem ser compreendidos com uma descrição clássica da magnetização macroscópica resultante. Dessa forma, em termos clássicos pode-se visualizar a RMN por núcleos com spin nuclear $\vec{I}$, sendo o momento angular $\vec{L}=\hbar \vec{I}$ e momento magnético $\vec{\mu}=\gamma \vec{L}=\gamma \hbar \vec{I}$, submetidos a um campo magnético estático externo $\vec{B}_{0} \hat{z}$ e excitados por um pulso de RF com campo oscilante $\vec{B}_{1}(t)$.

Imersos em um campo magnético estático $\vec{B}_{0} \hat{z}$, cada momento magnético nuclear $\vec{\mu}_{i}$ precessiona ao redor desse campo com frequência angular $\vec{\omega}_{0}$, que depende da constante 
magnetogírica do isótopo $\gamma$ e de $\vec{B}_{0}$

$$
\vec{\omega}_{0}=\gamma \vec{B}_{0}
$$

Cada isótopo apresenta uma constante magnetogírica diferente, por exemplo, para o hidrogênio $\left({ }^{1} H\right)$ é $42,58 \mathrm{MHz} / \mathrm{T}$ e para o carbono $\left({ }^{13} \mathrm{C}\right) 10,71 \mathrm{MHz} / \mathrm{T}$. Desse modo, cada isótopo tem uma frequência de precessão única, conhecida também como frequência de Larmor $\left(\vec{\omega}_{0}\right)$. Para a grande maioria das aplicações da RMN a frequência de ressonância $\left(\vec{\omega}_{0} / 2 \pi\right)$ situa-se na faixa das frequências de rádio $(\mathrm{MHz})$.

A magnetização macroscópica observada, $\vec{M}_{0}$, surge do pequeno excesso de núcleos com $\vec{\mu}$ na direção de $\vec{B}_{0} \hat{z}$, dada pela soma vetorial dos momentos magnéticos individuais $\left(\vec{\mu}_{i}\right)$

$$
\vec{M}_{0}=\sum_{i} \vec{\mu}_{i}
$$

No sistema de coordenadas de laboratório $(x, y, z)$, a descrição da RMN envolve movimentos tridimensionais complexos, que costumam ser simplificados com a descrição no sistema de coordenadas girantes. ${ }^{15}$ Neste sistema, o plano xy gira em torno do eixo z com uma frequência angular da ordem da frequência de Larmor $\left(\omega_{\text {ref }} \approx \omega_{0}\right)$, onde escolhemos $\omega_{\text {ref }}=-\omega_{1}$ do campo oscilante $\vec{B}_{1}$ do pulso de RF. Esses eixos são definidos por $\left(x^{\prime}, y^{\prime}, z\right)$. A diferença entre as duas frequências é denominada frequência de offset $\Omega=\omega_{\text {ref }}-\omega_{0}$. Nesse sistema de coordenadas girantes, após um pulso, a magnetização retorna ao equilíbrio descrita pelas equações de Bloch

$$
\left\{\begin{array}{l}
M_{x^{\prime} y^{\prime}}=M_{o} e^{-t / T_{2}} \\
M_{z}=M_{o}\left(1-e^{-t / T_{1}}\right)
\end{array}\right.
$$

com os tempos de relaxação spin-rede (longitudinal) $T_{1}$ e spin-spin (transversal) $T_{2}$.

Uma revolução na sensibilidade da RMN começou em 1966 com a proposta de R. Ernest e W. Anderson ${ }^{11}$ de utilizar pulsos curtos de RF para excitar o sistema de spins, seguido da aquisição de seu sinal transiente e processamento com Transformada de Fourier. A Fig. 1.1 apresenta um esquema da aquisição de sinais com a RMN pulsada. Iniciando no equilíbrio térmico, o pulso de RF com duração típica da ordem de $\tau=10 \mu \mathrm{s}$, retira a magnetização do equilíbrio em $\hat{z}$ e coloca no plano transversal $x y$, onde bobinas de detecção adquirem o 


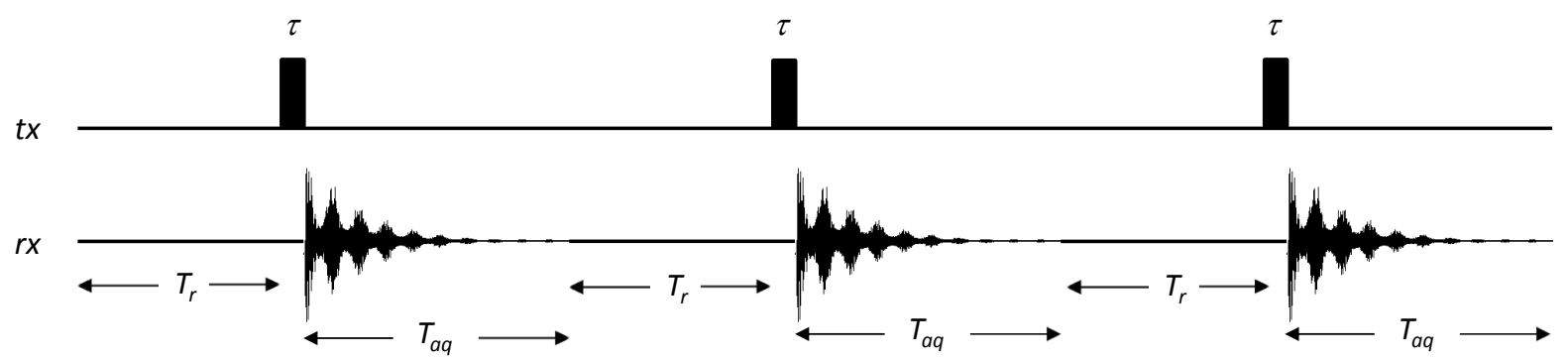

Figura 1.1- Esquema da técnica de RMN pulsada com intervalos de tempos entre pulsos $T_{p}=T_{a q}+T_{r}$, onde $T_{a q}$ é o tempo de aquisição, $T_{r}$ tempo de reciclo, $\tau$ o tempo do pulso $\left(\tau<<T_{p}\right)$. Após cada pulso aplicado, um sinal transiente do retorno da magnetização ao equilíbrio é observado, denominado Free Induction Decay.

Fonte: Elaborada pelo autor.

sinal transiente em $T_{a q}$ do retorno da magnetização ao equilíbrio, denominado Free Induction Decay (FID).

O tempo de aquisição varia entre milissegundos a poucos segundos dependendo dos tempos da relaxação da amostra. Em seguida, um tempo de reciclo $T_{r}$ é acrescentado, para que toda a magnetização retorne ao estado de equilíbrio antes do próximo pulso, sendo recomendado o tempo de repetição $T_{p}=T_{a q}+T_{r}=5 T_{1}$ para que $99,33 \%$ da magnetização tenha retornado ao equilíbrio.

A baixa intensidade dos sinais de RMN torna necessária a realização de centenas a milhares de aquisições na obtenção de uma razão s/r satisfatória. Devido a distribuição gaussiana do ruído térmico - 'ruído branco' - o aumento na razão s/r dos sinais não é linear, mas depende da raiz quadrada do número de aquisições, $s / r \propto n^{1 / 2}$. Assim, para dobrar a $s / r$ de um sinal é preciso multiplicar por quatro o número de aquisições realizadas. Esse processo de acumulação dos sinais eleva consideravelmente o tempo total de aquisição, principalmente em núcleos com baixa sensibilidade e tempos de relaxação elevados, agravados se impor $T_{p} \geq 5 T_{1}$.

A aplicação de alternâncias de fase nos pulsos e no receptor dessas sequências possibilita a seleção e cancelamento de componentes no FID ao longo das médias realizadas. Além disso, uma gama enorme de pulsos e modos de aquisição possibilitam as diversas aplicações da RMN, que são baseadas no método proposto por Ernest e Anderson de adquirir o sinal de precessão livre após os pulsos e obter o espectro pelo processamento com a Transformada de Fourier. 


\subsection{Precessão Livre no Estado Estacionário}

Um fenômeno fundamental na RMN é a formação dos Ecos de Spins, que foram pioneiramente descritos por E.L. Hahn em 1950. ${ }^{19}$ Hahn notou que ao aplicar dois pulsos idênticos espaçados por um curto intervalo de tempo, após esse mesmo intervalo um eco era formado no sinal adquirido. Em 1954, H.Y. Carr e E.M. Purcell demonstraram as vantagens de se aplicar o segundo pulso com dobro de intensidade, no caso $\frac{\pi}{2}-\tau-\pi$, estabelecendo a aplicação periódica de pulsos de $180^{\circ} .{ }^{20}$ Logo em 1958, S. Meiboom e D. Gill ${ }^{21}$ notaram a importância das fases escolhidas, estabelecendo os princípios fundamentais da sequência de pulsos CarrPurcell Meiboom-Gill (CPMG) e dos métodos spin-eco.

Os ecos de spins são gerados pelo restabelecimento da coerência de fase de um grupo de isocromatas $^{\ddagger}$ do sistema e o número de ecos produzidos $\left(N_{\text {ecos }}\right)$ é diretamente ligado ao número de pulsos aplicados ${ }^{22-24}$

$$
N e \cos =\frac{3^{N-1}-1}{2}
$$

onde $\mathrm{N}$ é o número de pulsos. Sequências com múltiplos pulsos de RF em intervalos de tempos menores que os tempos de relaxação resultam na geração de múltiplos ecos e FID, que se sobrepõem construtiva e destrutivamente, gerando complexos padrões de interferência.

O primeiro relato da observação de um estado estacionário da magnetização devido a aplicação periódica de pulsos de RF foi publicado em 1951 por R. Bradford et al. ${ }^{25}$ Em 1958, H.Y. Carr publicou um artigo completo ${ }^{26}$ relacionando o fenômeno com os ecos de Hahn ${ }^{19}$, demonstrando a formação do estado estacionário com as equações de Bloch e discutindo a utilização da Steady State Free Precession (SSFP) - em português Precessão Livre no Estado Estacionário - para melhorar a razão $\mathrm{s} / \mathrm{r}$ dos sinais.

Em 1966 R.R. Ernst e W.A. Anderson propuseram a utilização de RMN pulsada com a análise pela Transformada de Fourier para aumentar a sensibilidade de experimentos de $\mathrm{RMN}^{11}$. Nesse trabalho foi avaliado o tempo de repetição dos pulsos $\left(T_{p}\right)$, mostrando que quando $T_{p}$ é da ordem de $T_{2}$ o estado SSFP é formado, gerando anomalias de fase e amplitude

\footnotetext{
$\ddagger$ Isocromatas representam a contribuição para a magnetização macroscópica de um grupo de spins numa faixa de frequência de precessão $\Omega$.
} 
nos espectros adquiridos. Para evitar as distorções e otimizar a razão s/r, apresentam o cálculo do ângulo ótimo para o caso de $T_{p}<T_{1}$, conhecido atualmente como 'Ângulo de Ernest'.

R. Freeman e H.D.W. Hill em $1971^{27}$ propuseram o método Scrambled Steady-State (SSS) para eliminação das anomalias de fase e amplitude nos espectros SSFP. Esse método era baseado na adição de um incremento randômico no tempo de repetição entre os pulsos, que após muitas médias eliminava a componente eco do sinal e minimizava as distorções espectrais. No mesmo ano, A. Schwenk ${ }^{28}$ propôs uma técnica alternativa baseada na variação do offset de frequência, realizando apenas quatro experimentos em offset diferentes. Essa técnica também possibilitou minimizar as anomalias e foi denominada Quadriga Fourier Transform (QFT). No ano seguinte, Ernest percebeu que o método QFT poderia ser executado de maneira simplificada com blocos de aquisição com alternância de fase com incremento $0, \frac{\pi}{2}, \pi$ e $\frac{3 \pi}{2}$. A proposta de Ernest, apesar de promissora, não foi desenvolvida na época. ${ }^{29}$

Na mesma época, foi proposta a sequência Driven Equilibrium Fourier Transform (DEFT) para aumentar sensibilidade dos experimentos, que também coloca a magnetização em um estado de equilíbrio, através do ciclo de pulsos $90^{\circ}{ }_{x}-180^{\circ}{ }_{y}-90^{\circ}{ }_{x} \cdot{ }^{30}$ Outra técnica que deriva da SSFP são as sequências para excitação seletiva do tipo DANTE. ${ }^{31}$ Essas técnicas multipulsos de aquisição rápidas geraram diversas outras, com destaque na área de geração de imagens em MRI, devido a capacidade de aumentar a razão s/r em curto tempo de aquisição e forte sensibilidade ao fluxo ${ }^{32}$, como nas sequências FLASH, FISP e bSSFP. ${ }^{33}$

De crescente importância nas últimas décadas vale citar as sequências no estado estacionário em baixa resolução, utilizadas principalmente na determinação dos tempos de relaxação, como a Continuous Wave Free Precession (CWFP) ${ }^{34}$, Driven-Equilibrium CPMG $(\mathrm{DECPMG})^{35}$ e Split $180^{\circ} .{ }^{36}$

\section{SSFP em São Carlos}

No grupo de pesquisa da Embrapa Instrumentação, São Carlos-SP, os estudos com sequências rápidas de pulsos SSFP, iniciou com Rodrigo B.V. Azevedo, Luiz A. Colnago e Mario Engelsberg no desenvolvimento de técnicas para quantificação rápida de sementes oleoginosas através da amplitude do sinal SSFP 37 em espectrômetros de baixa resolução LR-RMN (Low resolution 
NMR) no final da década de 90. Nesses primeiros anos a formulação do estado Continouos Wave Free Precession (CWFP) foi apresenta com sua forte sensibilidade ao fluxo e ganho na razão $s / r$ frente aos métodos spin-ecos em LR-RMN. ${ }^{38}$

Em 2005, T. Venâncio et al. introduziram ${ }^{39}$ uma variação do método de Kronenbitter e Schwenk ${ }^{40}$ para realizar medidas simultâneas dos tempos de relaxação $T_{1}$ e $T_{2}$ pela CWFP. Para a medida dos tempos de relaxação faz-se a aquisição do sinal de RMN desde o primeiro pulso até atingir o estado estacionário de onda contínua CWFP. Com as amplitudes dos sinais inicial $\left(M_{0}\right)$ e no estado estacionário $\left(M_{s s}\right)$ e a constante de tempo $T^{*}$ entre o estado quasi estacionário e estacionário, calcula-se em um único experimento os tempos de relaxação $T_{1}$ e $T_{2}$, ver seção 2.5.1.

Logo em 2006 é apresentado o método para determinação de coeficientes de difusão térmica pela CWFP ${ }^{41,42}$, abrindo caminho para os trabalhos voltados para aplicações industriais em sistemas de fluxo on-line e in-line ${ }^{43-45}$ em diversas aplicações. ${ }^{46}$

Para amostras com $T_{1} \approx T_{2}$ a diferença de amplitude do sinal CWFP entre o estado quasi estacionário e estacionário é próxima de zero, o que dificulta a obtenção do $T^{*}$. Essa amplitude pode ser ampliada através da sequência CP-CWFP ${ }^{47}$ (Carr-Purcell - Continuous Wave Free Precession).

Métodos SSFP em alta resolução foram explorados em alguns trabalhos, principalmente para aumentar a razão $\mathrm{s} / \mathrm{r}^{48-51}$ e na supressão de sinais de solventes. ${ }^{52,53}$ Esta Tese de doutorado iniciou nesse contexto de desenvolvimento dos métodos SSFP na Embrapa Instrumentação, onde uma série de desafios em alta e baixa resolução poderiam ser analisados com a introdução de alternância e incremento de fase, similar a resultados recentes da literatura em NQR e MRI. ${ }^{54-56}$

\subsection{Motivação e objetivos}

A RMN apresenta uma intrínseca baixa sensibilidade quando comparada com outras técnicas espectroscópicas, necessitando a realização de um grande número de médias do sinal. A intensidade do sinal cresce com o número de núcleos $N_{\text {nuc. }}$ o quadrado da campo magnético 
externo $B_{0}^{2}$ e a diminuição da temperatura da amostra T, como na equação ${ }^{57}$

$$
\operatorname{Sinal}_{R M N} \propto \text { const. } \frac{N_{\text {nuc. }} B_{0}^{2}}{T}
$$

onde a constante (const.) representa os fatores advindos da eficiência da instrumentação. Uma vez que o ruído térmico cresce com a raiz quadrada e o sinal com o quadrado de $B_{0}$, o fator significativo é que a razão $s / r$ cresce com $B_{0}^{3 / 2}$. A equação 1.3.1 torna claro que para aumentar a intensidade do sinal de RMN, desenvolver a instrumentação e aumentar a intensidade do campo magnético externo $B_{0}$ são prioritários, uma vez que diminuir a temperatura e concentração da amostras são fatores limitantes em diversas situações práticas.

Magnetos permanentes e eletroímãs supercondutores evoluíram imensamente desde os primórdios da RMN, gerando sistemas magnéticos comerciais com campos magnéticos cada vez mais intensos e homogêneos. Eletroímãs supercondutores produzem campos mais estáveis e homogêneos, funcionando em baixas temperaturas com o resfriamento por líquidos criogênicos, de modo que o custo dos magnetos supercondutores representam bem mais do que $50 \%$ do custo total de um equipamento de RMN. ${ }^{\S}$

De modo geral, o tempo total de um experimento será dado pelo número de sinais acumulados a se realizar vezes o tempo de cada aquisição. O tempo de cada aquisição é estabelecido de acordo com os tempos de relaxação do composto em estudo, que variariam desde poucos milissegundos até minutos.

A Tabela 1.1 apresenta uma comparação da sensibilidade e abundancia natural para os núcleos de ${ }^{1} \mathrm{H},{ }^{13} \mathrm{C},{ }^{15} \mathrm{~N}$ e ${ }^{57} \mathrm{Fe}$. Estimando valores do tempo de relaxação e número de médias para obter uma boa razão $s / r$, temos uma estimativa do tempo total para adquirir espectros desses núcleos. A tabela evidência que quanto menor a sensibilidade e abundância natural dos núcleos, mais médias são necessárias na aquisição dos sinais e o tempo experimental total em alguns casos tornam os experimentos inviáveis.

A aquisição de sinais de ${ }^{1} H$ não é um grande problema uma vez que a receptibilidade ${ }^{\mathbb{I}}$ é

$\S$ Uma tendência dos dias atuais são os espectrômetros de bancada construídos com imãs permanentes com alto grau de homogeneidade. ${ }^{58}$ Essa nova geração de magnetos já atinge 1 Tesla de campo e permitem a realização de experimentos de alta resolução antes só realizados em magnetos supercondutores.

ฯ Medida do quão fácil é medir o sinal de um núcleo, definido pelos fatores da abundância natural e sensibilidade. 
Tabela 1.1- Tabela apresenta alguns fatores que influenciam no tempo total de aquisição em RMN. O tempo experimental é estimado de acordo com a abundância natural, sensibilidade e influenciado pelo tempo de relaxação dos núcleos.

\begin{tabular}{cccccc}
\hline Núcleo & $T_{1}$ & Abund. (\%) & sensib. & $\mathrm{n}^{0}$ médias & Tempo exp. \\
\hline${ }^{1} \mathrm{H}$ & $\sim 3 \mathrm{~s}$ & 99,98 & 1,0 & 32 & 2 min \\
${ }^{13} \mathrm{C}$ & $\sim 10 \mathrm{~s}$ & 1,11 & 0,016 & 2048 & 6 horas \\
${ }^{15} \mathrm{~N}$ & $\sim 15 \mathrm{~s}$ & 0,37 & 0,001 & 4096 & 20 horas \\
${ }^{57} \mathrm{Fe}$ & $\sim 50 \mathrm{~s}$ & 2,19 & 0,00003 & 32768 & 18 dias!
\end{tabular}

Fonte: Elaborada pelo autor.

relativamente alta quando comparada com outros núcleos e o tempo de relaxação não é tão longo. Normalmente espectros em alta resolução de ${ }^{1} H$ com boa razão s/r podem ser obtidos em questão de minutos.

Para outros núcleos com menor sensibilidade, como por exemplo o ${ }^{13} C$, a situação é um pouco mais complicada. Sua sensibilidade é 64 vezes menor que a do ${ }^{1} H$, principalmente devido sua baixa abundância natural e menor constante giromagnética. Essa baixa sensibilidade faz com que um número maior de médias seja requerido para obtermos espectros com boa razão $\mathrm{s} / \mathrm{r}$. Além disso, a relaxação típica de núcleos de ${ }^{13} \mathrm{C}$ pode ser da ordem de poucos segundos até dezenas de segundos em alguns casos.

Outro exemplo é o núcleo de ${ }^{15} N$, que estimando um tempo de relaxação da ordem de 15 segundos, ao realizar 4096 médias e aguardando pouco mais que $T_{1}$ entre cada aquisição, o tempo total para realizar essa aquisição chega a superar 20 horas. Os altos custos de manutenção de um equipamento de RMN torna inviável realizar experimentos com duração tão longa, de modo que novos métodos para aquisição rápida são altamente desejáveis.

A literatura apresenta uma gama enorme de técnicas que visam reduzir o tempo total de experimentos em RMN. Pode-se reduzir esse tempo melhorando a instrumentação utilizada - campos magnéticos intensos, melhor detector, etc - enriquecendo ou concentrando mais sua amostra, ou ainda através de diferentes técnicas modernas por transferência de polarização, tais como DNP dynamic nuclear polarization, optical pumping, parahidrogen-induced polarization. ${ }^{57}$

Apesar do sucesso em aumentar até centenas de vezes a intensidade do sinais, essas técnicas requerem a adição de substâncias químicas nas amostras ou da utilização de acessórios sofisticados, não sendo técnicas de uso geral. 
A sequência de Precessão Livre no Estado Estacionário (SSFP) é baseada na ideia simples de reduzir os tempos entre aquisições, possibilitando realizar um número enorme de médias em um curto intervalo de tempo. Essa via alternativa para aquisição rápida de sinais reduz drasticamente a resolução dos espectros e introduz uma série de anomalias de fase e amplitude, sendo parte deste trabalho propor soluções na redução dessas anomalias.

Desta forma, neste trabalho exploramos as técnicas de Precessão Livre no Estado Estacionário em alta e baixa resolução introduzindo alternância e incremento de fases, motivados principalmente pelos recentes trabalhos realizados por T. Rudakov na Ressonância Quadrupolar Nuclear ${ }^{59}$ e de B. Hargreades na geração de imagens ${ }^{55}$, utilizando SSFP com esquemas de fases na geração de perfis de excitação uniforme com ótima razão s/r. Além disso, métodos de processamento de dados como Filter Diagonalization Method (FDM) ou Krylov Basis Diagonalization Method (KBDM) e Transformada Inversa de Laplace (ILT) foram desenvolvidos e aplicados em conjunto com as técnicas SSFP.

Podemos dividir os objetivos deste trabalho em três seções principais:

- Distribuição dos tempos de relaxação CWFP: Proposta de um método para análise da distribuição dos tempos de relaxação a partir de sinais de RMN de baixa resolução utilizando as sequências rápidas tipo do CWFP com alternância de fase;

- SSFP com alternâncias e incremento de fase: Desenvolver métodos SSFP com alternância e incremento de fase em alta resolução para supressão das anomalias de fase e amplitude nos espectros de núcleos de baixa sensibilidade;

- KBDM para processamento de sinais: Desenvolvimento do KBDM (Krylov Basis Diagonalization Method) para processamento de sinal de RMN como via alternativa a Transformada de Fourier e a Transformada Inversa de Laplace. 



\section{Capítulo 2}

\section{Magnetização no Estado Estacionário}

Neste capítulo, iniciamos descrevendo a evolução de um sistema de spin $1 / 2$ não-interagente na presença de campos $\vec{B}_{0}(\vec{r})$ e $\vec{B}_{1}(\vec{r}, t)$ inomogêneos, assumindo uma distribuição espacial onde o campo magnético estático aponta na direção $\hat{z}$. Em seguida, as seções apresentam propriedades da magnetização no estado estacionário, com a dependência da amplitude, do tempo transiente e o efeito da alternância de fase, através da descrição analíticas e por integração numérica das equações de Bloch. Por fim, descrevemos as sequências de pulsos em estruturas periódicas em baixa resolução, apresentando detalhes das sequências de pulsos Continuous Wave Free Precession (CWFP), Split 180 e Driven-Equilibrium CPMG (DECPMG).

\subsection{Evolução da magnetização em campos magnéti- cos inomogêneos $\vec{B}_{0}(\vec{r})$ e $\vec{B}_{1}(\vec{r}, t)$}

Sequências de pulsos em RMN apresentam dois tipos de eventos básicos, pulsos de RF e evolução livre da magnetização. O efeito desses eventos podem ser descritos por matrizes de rotação no espaço tridimensional $3 \times 3$ no referencial de coordenadas girante. ${ }^{60-62}$ Assim a evolução do vetor magnetização $\vec{M}(t)=\left(M_{x}, M_{y}, M_{z}\right)$ para uma sequência de pulsos qualquer 
pode ser descrita em termos da multiplicação de $n$ matrizes de rotação

$$
\vec{M}(t)=\prod_{i=1}^{n} R_{\hat{\eta}_{i}}\left(\epsilon_{i}\right) \vec{M}(0)
$$

onde $\vec{M}(0)=\left(0,0, M_{0}\right)$ é a magnetização no equilíbrio térmico e as matrizes $R_{\hat{\eta}_{i}}\left(\epsilon_{i}\right)$ aplicam rotações de ângulo $\epsilon_{i}$ ao redor do eixo $\hat{\eta}_{i}$.

Considerando um sistema de spins imerso em um campo magnético constante $\vec{B}_{0} \hat{z}$ que possui magnetização resultante $\vec{M}_{0}$ na direção $\hat{z}$. A evolução da magnetização após aplicação de um pulso $\left(\theta_{\varphi}\right)$ pode ser descrita pelas equações de Bloch em termos da multiplicação das matrizes de rotação $R_{\Phi} \hat{E}(t) R_{\theta_{\varphi}}$, da forma

$$
\vec{M}(t)=R_{\Phi} \hat{E}(t) R_{\theta_{\varphi}} \vec{M}(0)+M_{0}\left(1-E_{1}(t)\right) \hat{z}
$$

onde $R_{\theta_{\varphi}}$ é a matriz do pulso de RF de ângulo $\theta$ e fase $\varphi, R_{\Phi}$ a matriz de precessão da magnetização e $\hat{E}$ a matriz de relaxação. $O$ termo $M_{0}\left(1-E_{1}(t)\right) \hat{z}$ precisa ser somado para considerar a magnetização criada em $\hat{z}$ durante a evolução no período $t$. As matrizes de rotação podem ser expressas da forma

$$
R_{\theta_{x}}=\left(\begin{array}{ccc}
1 & 0 & 0 \\
0 & \cos (\theta) & -\operatorname{sen}(\theta) \\
0 & \operatorname{sen}(\theta) & \cos (\theta)
\end{array}\right), R_{\Phi_{z}}=\left(\begin{array}{ccc}
\cos (\Phi) & -\operatorname{sen}(\Phi) & 0 \\
\operatorname{sen}(\Phi) & \cos (\Phi) & 0 \\
0 & 0 & 1
\end{array}\right)
$$

e

$$
\hat{E}(t)=\left(\begin{array}{ccc}
E_{2} & 0 & 0 \\
0 & E_{2} & 0 \\
0 & 0 & E_{1}
\end{array}\right), \vec{M}(t)=\left(\begin{array}{c}
M_{x} \\
M_{y} \\
M_{z}
\end{array}\right), \vec{M}(0)=\left(\begin{array}{c}
0 \\
0 \\
M_{0}
\end{array}\right)
$$

onde o ângulo de precessão é $\Phi=\Omega t$, com offset de frequência $\Omega=\omega_{0}-\omega_{r f}$, e os termos de relaxação $E_{1}(t)=\exp \left(-t / T_{1}\right)$ e $E_{2}(t)=\exp \left(-t / T_{2}\right)$. Note que a matriz $R_{\theta_{x}}$ é para pulsos com fase $x$, pulsos com outras fases utilizam suas respectivas matrizes de rotação. 


\section{Inomogeneidade de $\vec{B}_{1}$}

As equações apresentadas até aqui são para a situação $\Delta B_{0}=\left|\frac{\Omega}{\gamma}\right|=\left|\frac{\omega_{0}-\omega_{1}}{\gamma}\right|<<B_{1}$, ou seja, para excitação do pulso de RF praticamente uniforme ao longo do offset de frequências. Dessa forma todas as isocromatas de frequências ao longo da distribuição de $\vec{B}_{0}(\vec{r})$ sofrem pulsos com mesmo ângulo $\theta_{\varphi}$.

Em magnetos unilaterais ${ }^{60-64}$, como em sensores tipo MOUSE-NMR e magnetos para medidas well-logging da industria de petróleo, uma larga faixa de campos estático $\vec{B}_{0}$ é encontrada ao longo do objeto em estudo. Além disso, bobinas de superfície geram campos magnéticos dos pulsos de RF não homogêneos e a condição de Larmor $\omega_{r f}=\gamma B_{0}$ não é estabelecida em todo volume da amostra. Nessa situação, os campos magnéticos são altamente inomogêneos - grossly inhomogeneous fields - e temos $\Delta B_{0}>>B_{1}$. Assim, um pulso aplicado vai realizar rotação por ângulos diferentes nas regiões fora de ressonância e a magnetização resultante será composta pela soma das diversas contribuições ao longo do volume de detecção na região aproximadamente entre $B_{0}(\vec{r})=\omega_{r f} / \gamma \pm B_{1}{ }^{65}$

Para incluir esses efeitos na descrição, vamos supor a aplicação de um pulso de RF com amplitude $B_{1}$, frequência $\omega_{r f}$ e fase $\varphi$, da forma $\gamma B_{1 c}(t)=\omega_{1} \cos \left(\omega_{r f} t+\varphi\right)$. Para um dado deslocamento de offset em $\Delta B_{0}$, Fig. 2.1, a magnetização precessiona ao redor de um campo magnético efetivo, com amplitude

$$
B_{e f}=\sqrt{B_{1}^{2}+\Delta B_{0}^{2}}
$$

e ângulo $\theta$ em relação ao eixo $\hat{z}$

$$
\theta=\tan ^{-1}\left(\frac{B_{1}}{\Delta B_{0}}\right)
$$

sendo $\Delta B_{0}=B_{0}-\omega_{r f} / \gamma$. A Fig. 2.1 apresenta geometricamente esses ângulos da precessão da magnetização sobre efeito do campo magnético efetivo para pulso de RF com fase $\pi / 2$.

Assim, um pulso de duração $\tau$ aplica uma rotação efetiva, dada por

$$
\beta_{e f}=\gamma B_{e f} \tau
$$


a)

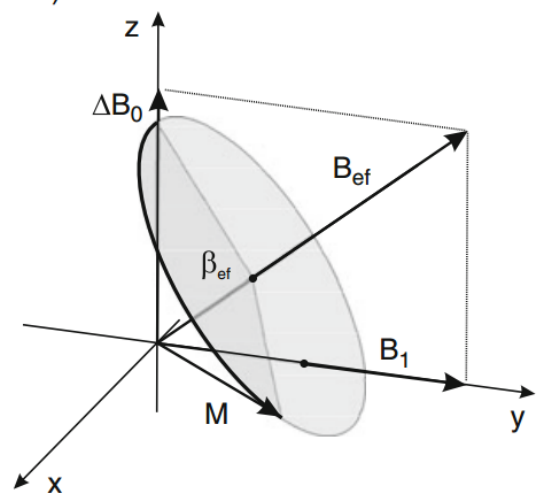

b)

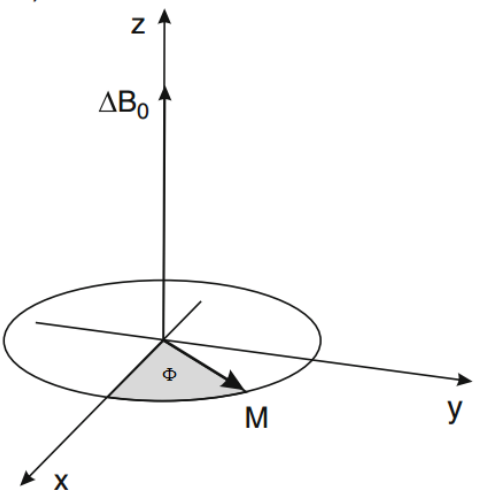

Figura 2.1- (a) Precessão do vetor magnetização ao redor do eixo da magnetização efetiva $B_{\text {ef }}$ durante a aplicação do pulso com amplitude $B_{1}$, fase $\pi / 2$ e frequência de offset $\gamma \Delta B_{0}$. (b) Precessão da componente durante a evolução livre.

Fonte: Adaptada de CASANOVA; PERLO; BLUEMICH. ${ }^{61}$

Determinado o ângulo de rotação efetivo, para determinar a rotação da magnetização em $\Delta B_{0}$ basta aplicar a matriz de rotação com ângulo $\beta_{\text {ef }}$ ao redor do campo efetivo $B_{e f}$. Por álgebra linear sabemos que essa rotação pode ser decomposta no produto das matrizes

$$
R_{B_{e f}}\left(\beta_{e f}\right)=\left[R_{g}(\theta, \varphi)\right]^{-1} R_{z}\left(\beta_{e f}\right)\left[R_{g}(\theta, \varphi)\right]
$$

onde $R_{z}\left(\beta_{e f}\right)$ é a matriz de rotação $\beta_{e f}$ no eixo $\hat{z}$ e $R_{g}(\theta, \varphi)$ matriz de rotação geral para os ângulos $\theta$ e $\varphi$

$$
R_{z}\left(\beta_{e f}\right)=\left(\begin{array}{ccc}
\cos \left(\beta_{e f}\right) & -\operatorname{sen}\left(\beta_{e f}\right) & 0 \\
\operatorname{sen}\left(\beta_{e f}\right) & \cos \left(\beta_{e f}\right) & 0 \\
0 & 0 & 1
\end{array}\right), \quad R_{g}(\theta, \varphi)=\left(\begin{array}{ccc}
\cos (\theta) \cos (\varphi) & \cos (\theta) \operatorname{sen}(\varphi) & -\operatorname{sen}(\theta) \\
-\operatorname{sen}(\varphi) & \cos (\varphi) & 0 \\
\operatorname{sen}(\theta) \cos (\varphi) & \operatorname{sen}(\theta) \operatorname{sen}(\varphi) & \cos (\theta)
\end{array}\right)
$$

Podemos agora retornar a equação 2.1.2, onde para o cálculo da evolução da magnetização basta substituir a matriz $R_{\theta_{\varphi}}$ por $R_{B_{e f}}\left(\beta_{e f}\right)$

$$
\vec{M}(t)=R_{\Phi} \hat{E}(t)\left[R_{B_{e f}}\left(\beta_{e f}\right)\right] \vec{M}(0)+M_{0}\left(1-E_{1}(t)\right) \hat{z}
$$

Através desse formalismo, a evolução da magnetização na presença de campos magnéticos inomogêneos $\vec{B}_{0}(\vec{r})$ e $\vec{B}_{1}(\vec{r}, t)$ pode ser calculada aplicando a sequência de rotações estabelecida pela sequência de pulsos e precessões livres. A integração numérica das contribuições de cada região da distribuição de campos magnéticos $\vec{B}_{0}$ e $\vec{B}_{1}$ fornecem no comportamento da 
magnetização macroscópica resultante. ${ }^{66}$

\subsection{Amplitude da magnetização no Estado Estacioná- rio}

As sequências de pulsos de precessão livre no estado estacionário, em inglês Steady State Free Precession (SSFP), são baseadas na aplicação de um trem de pulsos de mesma intensidade, ângulo $\theta_{\varphi}$ e separados por um intervalo constante de tempo $T_{p}$.

A Fig. 2.2 apresenta o esquema geral da sequência, onde $T_{p}=T_{a q}+T_{r}+\tau$, com $T_{a q}$ tempo de aquisição do sinal, $T_{r}$ tempo de reciclo e $\tau$ tempo do pulso aplicado. Utilizando o tempo entre pulsos menor que os tempos de relaxação $T_{p}<T_{1}, T_{2}$, após um tempo transiente um estado estacionário da magnetização se forma no período de precessão livre, permanecendo nesse estado enquanto for continuada a aplicação dos pulsos.

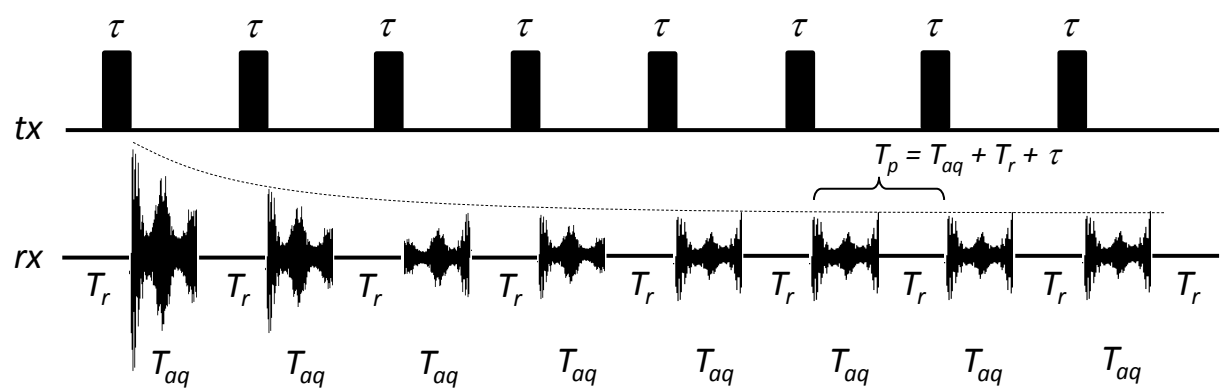

Figura 2.2- Sequência de pulsos com $T_{p}$ menor que os tempos de relaxação. A consequência de sequências de pulsos desse tipo é a estabilização de um estado estacionário da magnetização após um tempo transiente.

Fonte: Elaborada pelo autor.

A estabilização desse estado estacionário da magnetização é dependente de uma série de parâmetros experimentais, como o ângulo dos pulsos e suas fases $\left(\theta_{\varphi}\right)$, tempo entre pulsos $\left(T_{p}\right)$, offset de frequência $(\Omega)$, gradiente de campo; assim como parâmetros intrínsecos das amostras tais como os tempos de relaxação $T_{1}$ e $T_{2}$, que contribuem no tempo da formação do estado estacionário e sua amplitude.

No início da sequência de pulsos a magnetização passa por um período transiente e após $n$ pulsos atinge o estado estacionário de precessão livre. Nessa situação o vetor magnetização passa a realizar sempre o mesmo movimento em cada ciclo de pulso periódico em $T_{p}$. Em 
particular, a magnetização imediatamente antes do pulso $M^{-}$e imediatamente depois de receber o pulso $M^{+}$, estão relacionadas por (Fig. 2.3)

$$
\left\{\begin{array}{l}
\underbrace{\vec{M}\left[n\left(T_{p}+\tau\right)+\tau\right]}_{M^{+}}=\hat{R}_{\theta} \cdot \underbrace{\vec{M}\left[n\left(T_{p}+\tau\right)\right]}_{M^{-}} \\
\underbrace{\vec{M}\left[n\left(T_{p}+\tau\right)\right]}_{M^{-}}=\hat{R}_{\Phi} \hat{E} \cdot \underbrace{\vec{M}\left[n\left(T_{p}+\tau\right)+\tau\right]}_{M^{+}}+M_{0}\left(1-E_{1}\right) \hat{z}
\end{array}\right.
$$

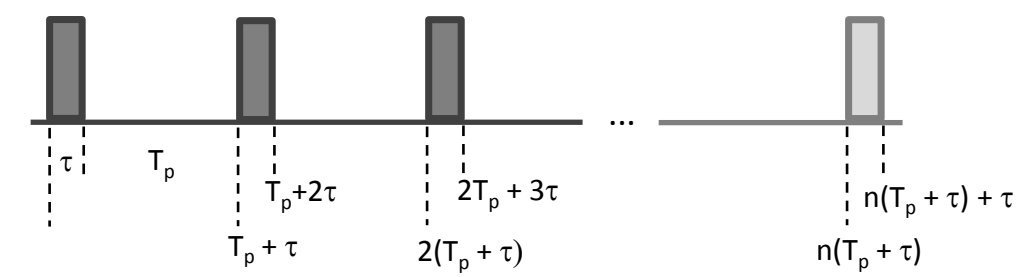

Figura 2.3- Tempos imediatamente antes e após os pulsos de RF com duração $\tau$ e espaçados por $T_{p}$. Fonte: Elaborada pelo autor.

Rearranjando as equações 2.2.1, temos

$$
M^{+}=\hat{R}_{\theta}\left(\hat{R}_{\Phi} E M^{+}\right)+\hat{R}_{\theta} M_{0}\left(1-E_{1}\right) \hat{z}
$$

Resolvendo o sistema para $\vec{M}^{+}=\left(M_{x}^{+}, M_{y}^{+}, M_{z}^{+}\right)($Cramer $)$as três componentes antes e depois do pulso são obtidas

$$
\begin{aligned}
M_{x}^{-} & =\frac{M_{0}\left(1-E_{1}\right)\left[E_{2} \operatorname{sen}(\theta) \operatorname{sen}(\Phi)\right]}{D} \\
M_{y}^{-} & =\frac{M_{0}\left(1-E_{1}\right)\left[E_{2} \operatorname{sen}(\theta) \cos (\Phi)-E_{2}^{2} \operatorname{sen}(\theta)\right]}{D} \\
M_{z}^{-} & =\frac{M_{0}\left(1-E_{1}\right)\left[1-E_{2} \cos (\Phi)-E_{2} \cos (\theta)\left(\cos (\Phi)-E_{2}\right)\right]}{D} \\
M_{x}^{+} & =M_{x}^{-} \\
M_{y}^{+} & =\frac{M_{0}\left(1-E_{1}\right)\left[\left(1-E_{2} \cos (\Phi)\right) \operatorname{sen}(\theta)\right]}{D} \\
M_{z}^{+} & =\frac{M_{0}\left(1-E_{1}\right)\left[E_{2}\left(E_{2} \cos (\Phi)\right)+\left(1-E_{2} \cos (\Phi)\right) \cos (\theta)\right]}{D}
\end{aligned}
$$


onde

$$
D=\left[\left(1-E_{1} \cos (\theta)\right)\left(1-E_{2} \cos (\Phi)\right)\right]-\left[\left(E_{1}-\cos (\theta)\right)\left(E_{2}-\cos (\Phi)\right) E_{2}\right]
$$

Essas equações foram pioneiramente derivadas por Ernst e Anderson. ${ }^{11}$ Pelas equações 2.2.6 e 2.2.7 podemos calcular o módulo do vetor magnetização no plano $x y$ logo após receber o pulso de RF

$$
\begin{aligned}
|M| & =\sqrt{\left(M_{x}^{+}\right)^{2}+\left(M_{y}^{+}\right)^{2}} \\
& =\frac{M_{0}|\operatorname{sen}(\theta)|\left(1-E_{1}\right)}{D} \sqrt{1+E_{2}^{2}-2 E_{2} \cos (\Phi)}
\end{aligned}
$$

Essas equações são válidas para qualquer valor de $T_{p}$. De fato, na SSFP temos $T_{p}<<T_{1}$ e $T_{2}$, portanto a exponencial pode ser expandida $E_{1,2}=\exp \left(-T_{p} / T_{1,2}\right) \approx 1-T_{p} / T_{1,2}$. Substituindo em 2.2.11 obtemos a seguinte expressão para o módulo da magnetização

$$
|M|=\frac{M_{0}|\operatorname{sen}(\theta)| \sqrt{2-2 \cos (\Phi)}}{(1+\cos (\theta))(1-\cos (\Phi))+(1-\cos (\theta)) 2 T_{1} / T_{2}}
$$

A Fig. 2.4 apresenta o comportamento das equação 2.2.12 em função do offset de frequência com $T_{p}=100 \mathrm{~ms}, T_{1}=5 \mathrm{~s}$ e $T_{2}=5 \mathrm{~s}$, em A) $\operatorname{com} \theta=90^{\circ}$ e B) $\theta=30^{\circ}$. Na parte superior apresentamos modulação das componentes real (preto), imaginária (vermelho) e módulo (azul) da magnetização, na parte inferior a fase dos sinais.

Note que a magnetização $|M| \rightarrow 0$ quando $\sqrt{2-2 \cos (\Phi)} \rightarrow 0$, ou seja a magnetização se anula para todo $\Phi=n 2 \pi$, onde $n$ é um número inteiro. Assim temos

$$
\begin{aligned}
\Phi=\Omega T_{p} & =n 2 \pi \\
\left(f^{\prime}-f_{0}\right) & =\frac{n}{T_{p}}
\end{aligned}
$$

de modo que iniciando pela posição do centro da janela espectral $f_{0}$ (posição do offset) as frequências $\left(f^{\prime}\right)$ múltiplas de $1 / T_{p}$ são suprimidas no espectro.

Voltando as equações 2.2 .6 e 2.2.7 podemos calcular o ângulo que a magnetização faz 

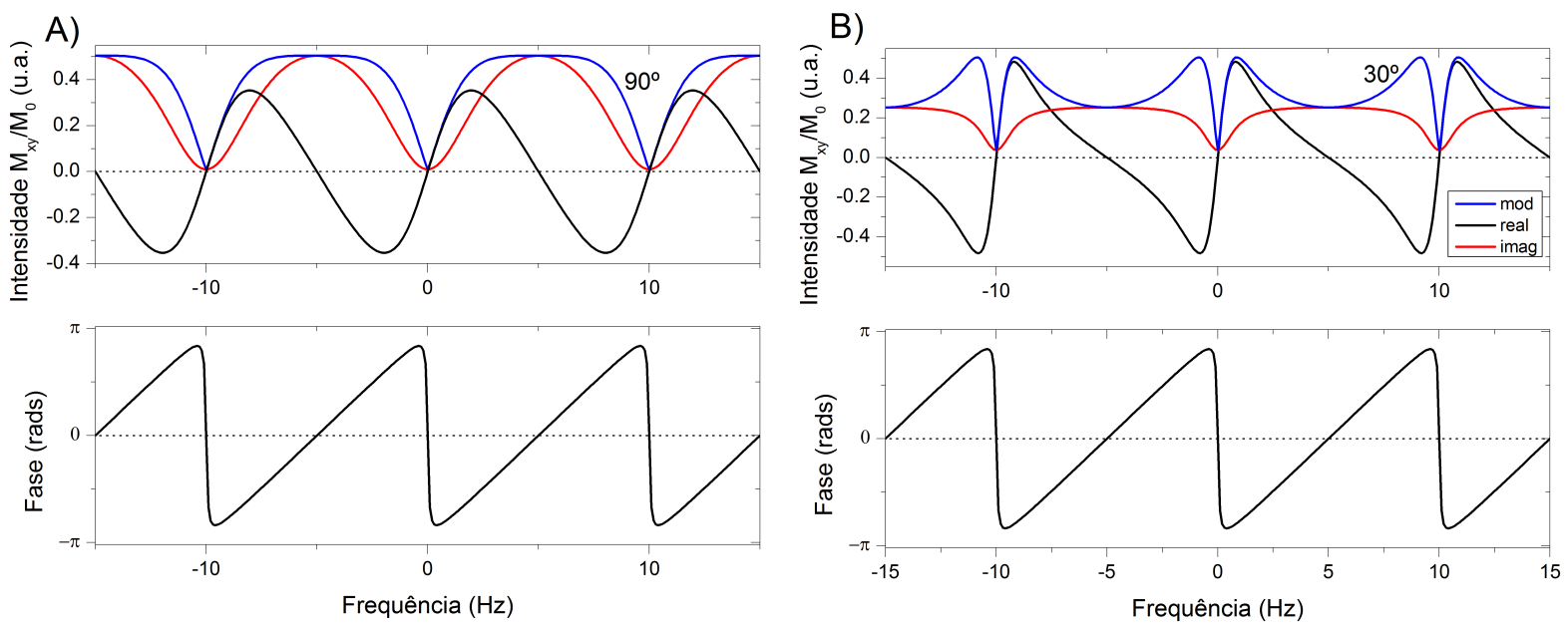

Figura 2.4- Sinais teóricos do comportamento da magnetização em função do offset de frequência com $T_{p}=$ $100 \mathrm{~ms}, T_{1}=5 \mathrm{~s} \mathrm{e} T_{2}=5 \mathrm{~s}$, em A) $\operatorname{com} \theta=90^{\circ}$ e B) $\theta=30^{\circ}$. Na parte superior componentes real (preto), imaginária (vermelho) e módulo (azul) da magnetização; abaixo a fase dos sinal.

Fonte: Elaborada pelo autor.

com o eixo $\hat{y}$ logo após a aplicação do pulso de RF

$$
\tan (\epsilon)=\frac{M_{y}^{+}}{M_{x}^{+}} \quad ; \quad \epsilon=\tan ^{-1}\left(\frac{E_{2}^{-1}-\cos (\Phi)}{\operatorname{sen}(\Phi)}\right)
$$

Pela equação 2.2.12 a amplitude é uma função do ângulo do pulso $\theta$, de $\Phi$ e da razão $T_{1} / T_{2}$, de modo que o máximo da amplitude do sinal pode ser obtido por $d|M| / d \Phi=0$, $\log 0$

$$
\cos (\theta)=\frac{T_{1} / T_{2}-\operatorname{sen}^{2}(\Phi / 2)}{T_{1} / T_{2}+\operatorname{sen}^{2}(\Phi / 2)}
$$
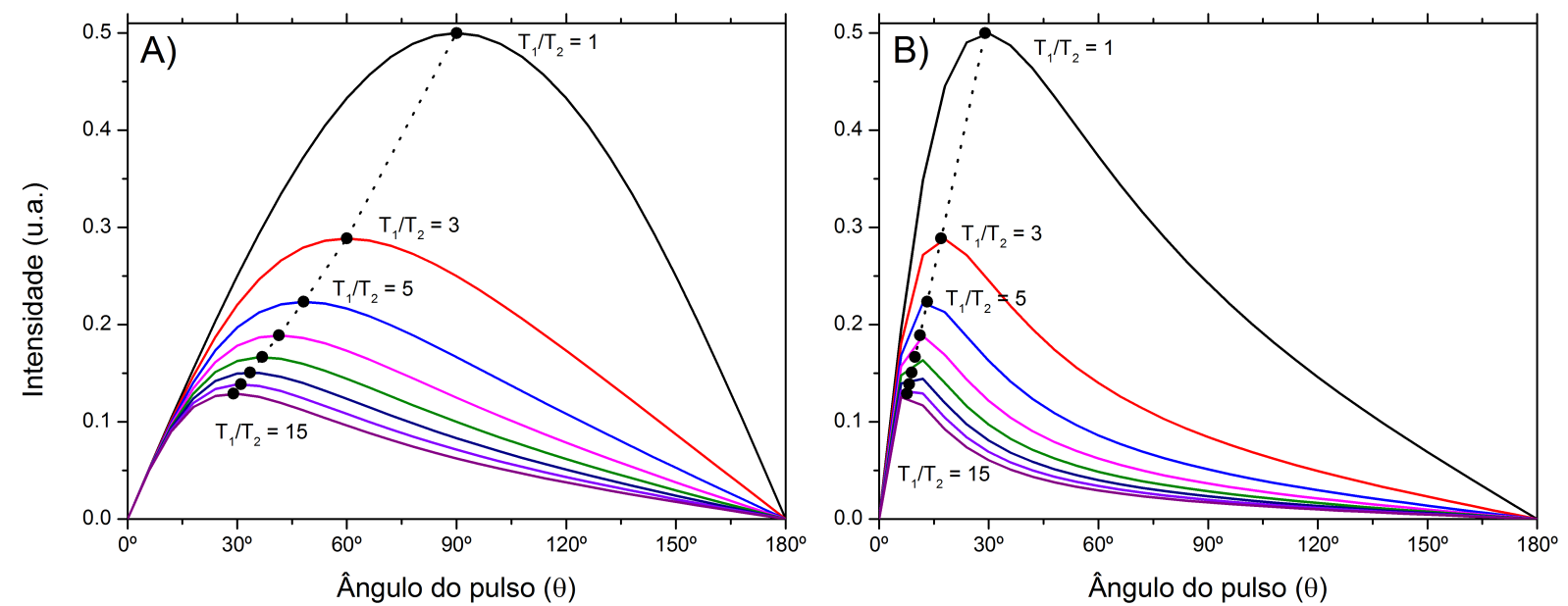

Figura 2.5- Amplitude da magnetização no estado estacionário em função do ângulo dos pulsos $\theta$ e da razão $T_{1} / T_{2}$ em A) para isocromatas com ângulo de precessão $\Phi=\pi$ e em B) $\Phi=\pi / 6$. Os pontos $(\bullet)$ e a linha tracejada denotam as posição de $\theta_{o p}$ ótimo para cada razão $T_{1} / T_{2}$.

Fonte: Elaborada pelo autor. 
A Fig. 2.5 apresenta a amplitude da magnetização no estado estacionário em função do ângulo dos pulsos $\theta$ e da razão $T_{1} / T_{2}$ em A) para isocromatas com ângulo de precessão $\Phi=\pi$ e em B) $\Phi=\pi / 6$. Os pontos $(\bullet)$ e a linha tracejada denotam as posição de $\theta_{o p}$ ótimo para razão $T_{1} / T_{2}$ entre 1 e 15 .

O sinal tem amplitude máxima para $\Phi=(2 n+1) \pi$, assim

$$
\cos \left(\theta_{o p}\right)=\frac{T_{1} / T_{2}-1}{T_{1} / T_{2}+1}
$$

Essa equação fornece o $\theta_{o p}$ ótimo para obter o máximo da amplitude de sinal da magnetização em função da razão $T_{1} / T_{2}$. Desse modo, pela equação 2.2.12 o máximo de intensidade da magnetização é

$$
|M| \leq \frac{M_{0}}{1+T_{1} / T_{2}}
$$

A amplitude dos picos ao longo de um espectro numa aquisição no estado estacionário vão apresentar amplitude máxima igual a metade da amplitude $M_{0}$ e os picos espectrais são modulados com amplitude dependente da razão $T_{1} / T_{2}$ de cada.

\subsection{Transiente ao Estado Estacionário}

A estabilização do estado estacionário da magnetização não é imediata após o início da aplicação dos pulsos de RF, como ilustra a Fig. 2.2. O objetivo dessa seção é calcular o tempo característico que a magnetização leva para estabelecer o estado estacionário.

Iremos aqui simplificar a descrição desse transiente seguindo apenas uma isocromata com frequência de offset $\Omega$, que após o pulso de RF sofre precessão livre por um tempo $T_{p}$, relaxando até a direção $-\hat{y}$. Essa condição é dada por $\Phi=\Omega T_{p}=(2 n+1) \pi$ e nos garante que no estado estacionário a magnetização imediatamente antes do pulso e imediatamente depois do pulso se encontra no plano $y z$.

Na Fig. 2.6 temos que após o início da aplicação de pulsos com ângulo $\theta$ a isocromata imediatamente antes $\left(M_{p}^{(a)}\right)$ e imediadamente depois $\left(M_{p}^{(d)}\right)$ da aplicação dos pulsos encontram-se no plano yz. No estado estacionário após aplicação de $n$ pulsos a magnetização antes e de- 
pois dos pulsos (componentes vermelhas) podem ser decompostas em termos da magnetização $M_{p}^{(a, d)}$ e $M_{w}$ que é a componente em $\hat{z}$ que não se altera.
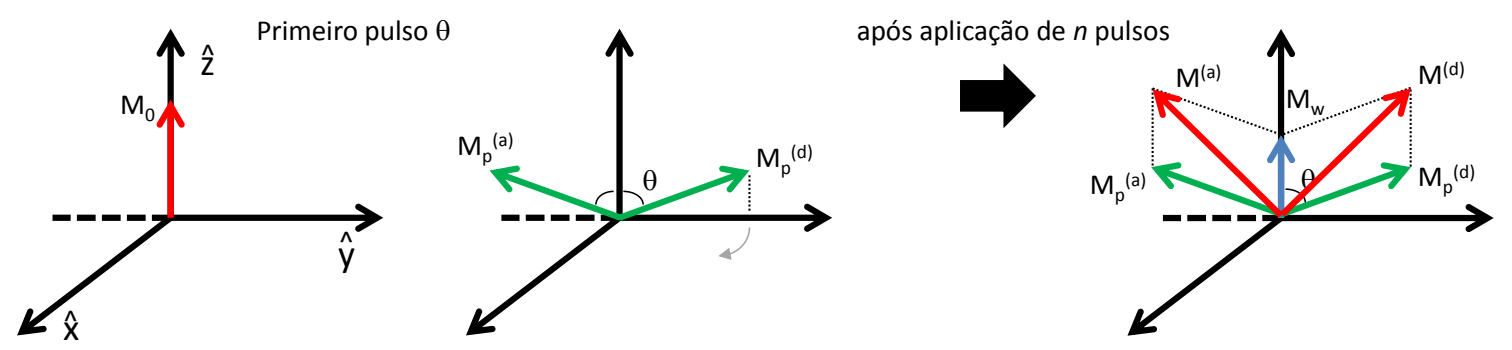

Figura 2.6- Após o início da aplicação de pulsos com ângulo $\theta$ a isocromata que precessiona com $\Phi=$ $(2 n+1) \pi$ imediatamente antes $\left(M_{p}^{(a)}\right)$ e imediadamente depois $\left(M_{p}^{(d)}\right)$ da aplicação dos pulsos encontram-se no plano $y z$. Após aplicação de $n$ pulsos a magnetização antes e depois dos pulsos (componentes vermelhas) podem ser decompostas em termos da magnetização $M_{p}^{(a, d)}$ e $M_{w}$.

Fonte: Elaborada pelo autor.

Essas componentes estão relacionadas por

$$
\left\{\begin{array}{l}
M_{z}^{a, d}=M_{w}^{a, d}+M_{p}^{a, d} \cos (\theta) \\
M_{t}^{a, d}=M_{p}^{a, d} \operatorname{sen}(\theta)
\end{array}\right.
$$

com

$$
M_{w}^{a, d}=M_{z}^{a, d}-M_{t}^{a, d} \frac{\cos (\theta)}{\operatorname{sen}(\theta)}
$$

Durante a precessão a isocromata sofre influência das relaxações, assim

$$
\left\{\begin{array}{l}
M_{z}^{d}=M_{z}^{a} \exp \left(-T_{p} / T_{1}\right)+M_{0}\left(1-E_{1}\right) \\
M_{t}^{d}=M_{t}^{a} \exp \left(-T_{p} / T_{2}\right)
\end{array}\right.
$$

Substituindo 2.3.3.1 em 2.3.2, temos $M_{w}^{d}=\left(M_{z}^{a} E_{1}+A\right)-M_{t}^{d} \cot (\theta)$, onde definindo $A=$ $M_{0}\left(1-E_{1}\right)$ e substituindo 2.3.1.1 no primeiro termo e 2.3.3.2 no segundo

$$
M_{w}^{d}=M_{w}^{a} E_{1}+M_{p}^{a} E_{1} \cos (\theta)+A-\left(M_{t}^{a} E_{2}\right) \cot (\theta)
$$

agora substituindo 2.3.1.2 no último termo

$$
M_{w}^{d}=M_{w}^{a} E_{1}+M_{0}\left(1-E_{1}\right)+M_{p}^{a}\left(E_{1}-E_{2}\right) \cos (\theta)
$$


Durante a relaxação a magnitude do vetor precessão é diminuída por $M_{p}^{a}=M_{p}^{d} E_{2}$. Desprezamos o efeito da relaxação durante o pulso de RF, no n-ésimo pulso temos

$$
M_{w(n)}^{a}=M_{p(n-1)}^{d}, \quad M_{p(n)}^{a}=M_{w(n-1)}^{d}
$$

que resulta nas relações

$$
\left\{\begin{array}{l}
M_{w(n+1)}^{a}=M_{p(n)}^{d}=M_{p(n)}^{a} E_{2} \\
M_{p(n+1)}^{a}=M_{w(n)}^{d}=M_{0}\left(1-E_{1}\right)+M_{w(n)}^{a} E_{1}+M_{p(n)}^{a}\left(E_{1}-E_{2}\right) \cos (\theta)
\end{array}\right.
$$

portanto

$$
\left\{\begin{array}{l}
M_{w(n+2)}^{a}=M_{0}\left(1-E_{1}\right) E_{2}+M_{w(n)}^{a} E_{1} E_{2}+M_{w(n+1)}^{a}\left(E_{1}-E_{2}\right) \cos (\theta) \\
M_{p(n+2)}^{a}=M_{0}\left(1-E_{1}\right)+M_{p(n)}^{a} E_{1} E_{2}+M_{p(n+1)}^{a}\left(E_{1}-E_{2}\right) \cos (\theta)
\end{array}\right.
$$

Assim, vale a equação de recursão

$$
M_{(n+2)}=M_{0}\left(1-E_{1}\right)+M_{(n+1)}\left(E_{1}-E_{2}\right) \cos (\theta)+M_{(n)} E_{1} E_{2}
$$

Inicialmente a magnetização transversal é nula e após um número $n$ de pulsos chega ao estado estacionário. Podemos simplificar com $\left(E_{1}-E_{2}\right)<<\left(E_{1} \cdot E_{2}\right)$, logo $M_{n+1} \approx M_{n}$

$$
M_{(n+2)}=M_{0}\left(1-E_{1}\right)+M_{(n)}\left\{\left(E_{1}-E_{2}\right) \cos \theta+E_{1} E_{2}\right\}
$$

onde $A=M_{0}\left(1-E_{1}\right)$ e $C=\left(E_{1}-E_{2}\right) \cos \theta+E_{1} E_{2}$. Nota-se que

$$
\begin{array}{ll}
M_{0}=0 & M_{1}=0 \\
M_{2}=A+A C & M_{3}=0 \\
M_{4}=A+A C+A C^{2} & M_{5}=0
\end{array}
$$


Temos assim as séries

$$
M_{(2 n)}=A \sum_{k=0}^{n-1} C^{k} \quad ; \quad M_{(2 n)}=\frac{A\left(1-C^{n}\right)}{1-C}
$$

que pode ser escrita como

$$
M_{(2 n)}=\left\{\frac{A}{1-C}\right\}[1-\exp (n \ln C)]
$$

Fazendo as aproximações $\exp \left(-T_{p} / T_{1,2}\right) \approx 1-T_{p} / T_{1,2}$

$$
\begin{aligned}
A=M_{0}\left(1-E_{1}\right) & \approx M_{0} \frac{T_{p}}{T_{1}} \\
1-C=1-\left(E_{1}-E_{2}\right) \cos \theta-E_{1} E_{2} & \approx T_{p}\left[\frac{1+\cos \theta}{T_{1}}+\frac{1-\cos \theta}{T_{2}}\right]
\end{aligned}
$$

Logo, temos

$$
M_{w}(t)=M_{p}(t)=\frac{M_{0} T_{1}}{\left[\frac{1+\cos \theta}{T_{1}}+\frac{1-\cos \theta}{T_{2}}\right]}\left[1-\exp \left(t / T^{*}\right)\right]
$$

onde

$$
T^{*}=\frac{2 T_{1} T_{2}}{T_{1}(1-\cos \theta)+T_{2}(1+\cos \theta)}
$$

$T^{*}$ é o tempo característico que a magnetização leva para estabelecer o estado estacionário na condição $\Phi=(2 n+1) \pi$. A relação 2.3.17 estabelece que o $T^{*}$ depende dos tempos de relaxação $T_{1}$ e $T_{2}$, além do ângulo $\theta$ do pulso. Evidentemente, alternâncias e incrementos de fase dentre outras situações não foram considerados nessas equações. Para o estudo dos demais parâmetros foram utilizadas simulações computacionais que serão apresentadas a frente.

\subsection{Alternância de fase no Estado Estacionário}

Recentemente, alguns métodos para supressão da componente eco na SSFP vem sendo propostos através da utilização de alternância e esquemas de fases nos pulsos e receptor. T. Rudakov et al. realizou uma série de estudos introduzindo diversas alternâncias de fases nas 
sequências SSFP em ressonância nuclear quadrupolar NQR - Nuclear Quadrupole Resonance - para detecção de ${ }^{14} N$ em entorpecentes e explosivos. ${ }^{54,59,67-69} \mathrm{Na}$ aquisição de imagens por RMN - Nuclear Magnetic Resonance Imaging - destacamos os trabalhos realizados por B. Hargreaves et al. que também vem introduzindo uma série de esquemas da fase com múltiplas aquisições SSFP na eliminação de artefatos nas imagens. ${ }^{55,56,70} \mathrm{Em}$ baixa resolução, alguns trabalhos relacionados são as técnicas Split $180^{\circ} 36$ e a mais recentemente introduzida PIETA (Phase Incremented Echo Train Acquisition) que consiste numa CPMG com incremento de fase utilizada para eliminar artefatos do sinal. ${ }^{71,72}$

Nesta seção, visando uma melhor compreensão do comportamento da magnetização nas sequências SSFP com diversos esquemas de fase, foram desenvolvidos códigos numéricos para simulação dos sinais. Os códigos desenvolvidos foram criados baseados nos métodos por somatória de isocromatas na integração das equações de Bloch ${ }^{55,73}$ e um artigo de ensino usando a mesmo método foi publicado na revista Química Nova. ${ }^{66}$

\subsubsection{Perfil de Excitação}

Nas referências ${ }^{23,24} \mathrm{~J}$. Henning apresenta um formalismo que permite calcular a amplitude de todos os ecos gerados em uma sequência de pulsos arbitraria. Entretanto, esse formalismo requer que a magnetização transversal seja nula antes de cada pulso. Claramente isso simplifica os cálculos, porém não permite uma investigação mais completa da formação do estado estacionário.

Uma maneira simples e direta para analisar as propriedades de diversos esquemas de fase aplicados na SSFP é através de simulações numéricas. As simulações aqui apresentadas são baseadas na somatória de isocromatas em uma distribuição de componentes inomogênea lorentziana, ver seção 3. Assim, para obter os perfis de excitação das sequências com diferentes alternâncias de fase, o procedimento que utilizamos foi simular a magnetização até o ponto onde se estabelece o estado estacionário, monitorando a amplitude da magnetização para diferentes valores de offset de frequência $\Omega=w_{r f}-w_{0}$.

A Fig. 2.7 apresenta o perfil de excitação simulado para três esquemas de fase em função do ângulo do pulso $\theta$ aplicado, com $T_{1}=T_{2}=1 \mathrm{~s}$ e $T_{p}=100 \mathrm{~ms}$. Na linha (a) da Fig. 2.7 
apresentamos o perfil de excitação da SSFP com fase constante. Esses resultados concordam muito bem com a descrição analítica da seção 2.2 e a literatura, apresentando supressão periódica em intervalos de frequência $1 / T_{p}$ e amplitude modulada pelo ângulo $\theta$ e tempos de relaxação $T_{1}$ e $T_{2}$, como na Fig. 2.4 .

(a)
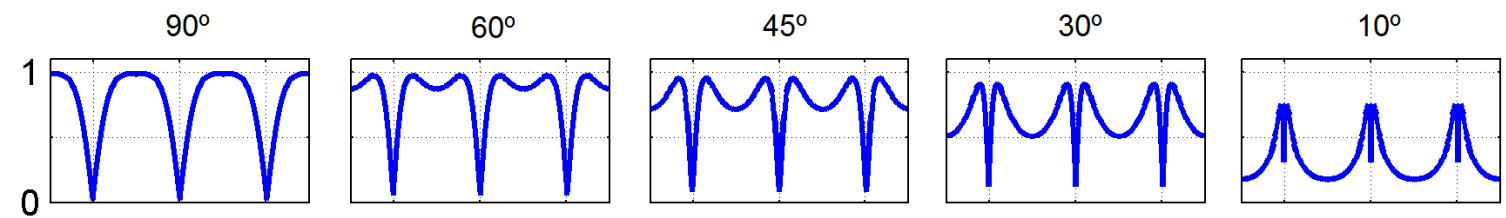

(b)
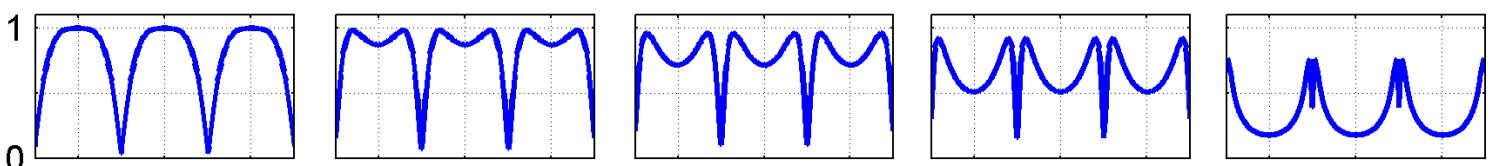

(c)
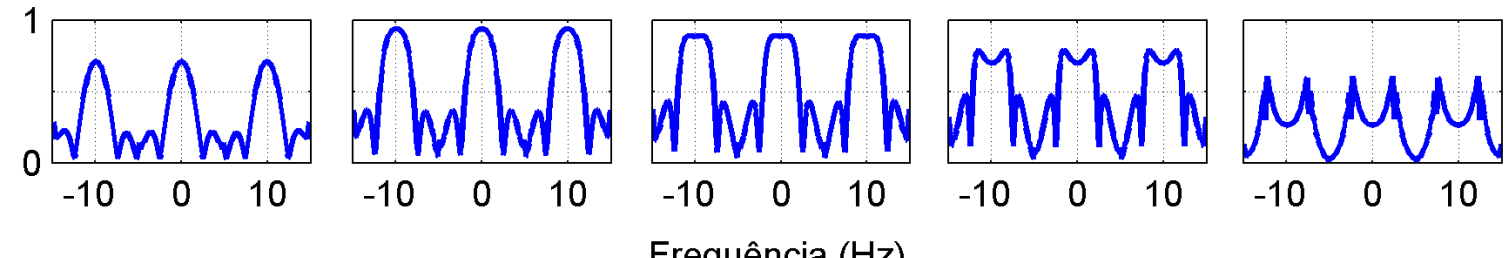

Frequência $(\mathrm{Hz})$

Figura 2.7- Sinais simulados do perfil de excitação para três esquemas de alternância de fase em função do ângulo do pulso $\theta$ aplicado, com $T_{1}=T_{2}=1 \mathrm{~s}$ e $T_{p}=100 \mathrm{~ms}$. A linha (a) apresenta o perfil de excitação da sequência SSFP com fase constante para diversos valores do ângulo do pulso $\theta$. As linhas (b) e (c) apresentam os perfis para as sequências SSFP com alternância de fase $(x,-x)$ e $(x, x,-x,-x)$ respectivamente.

Fonte: Elaborada pelo autor.

Ao introduzirmos pulsos com alternância ou incremento de fase na SSFP o perfil de excitação muda. A linha (b) da Fig. 2.7 apresenta o perfil de excitação para a SSFP com alternância de fase $(x,-x)$ - denominada aqui SSFPx-x - para diferentes valores do ângulo do pulso $\theta$. Nota-se que a supressão periódica continua com intervalos de frequência $1 / T_{p}$, entretanto, houve um deslocamento de offset nas frequências suprimidas, estando os máximos de sinal da SSFPx-x nos pontos de mínimos da SSFP.

Essas simulações numéricas das SSFP submetidas a diferentes alternâncias de fase mostramse de grande eficiência no estudo dos perfis de excitação gerados com ótima concordância experimental. Muitas não apresentam vantagens aos métodos que estamos trabalhando, como por exemplo o resultado apresentado na linha (c) da Fig. 2.7, do perfil de excitação de uma SSFP com alternância de fase $(\mathrm{x}, \mathrm{x},-\mathrm{x},-\mathrm{x})$ proposta por Rudakov ${ }^{54}$ para $N Q R$. Nota-se que mais regiões de supressão de sinais são introduzidas, possuindo duas regiões com amplitude de excitação diferentes no eixo das frequências. 


\section{SSFP com incremento linear de fase}

É interessante notar que o perfil de excitação da sequência com alternância $(x,-x)$ apresenta-se deslocados no eixo da frequência em relação ao perfil da sequência com fase constante. No $\operatorname{artigo~}^{74,75}$ foi mostrado que o deslocamento $\delta f$, em $\mathrm{Hz}$, é proporcional ao incremento de fase utilizado

$$
\delta f=\frac{\delta \varphi}{2 \pi T_{p}}
$$

onde $\delta \varphi$ é o incremento constante de fase na sequência de pulsos. Definimos assim a sequência SSFP com incremento constante de fase $\delta \varphi$, denominada aqui SSFPdx, onde o termo $d x$ representa o incremento $\delta \varphi$. A fase do $n$-enésimo pulso dessa sequência é dada por

$$
\varphi(n)=\varphi_{0}+(n-1) \delta \varphi
$$

com $n=1,2,3, \ldots, N_{f}$, onde $N_{f}$ o último pulso da sequência. A fase de um pulso ao próximo é sempre incrementado pelo valor contante $\delta \varphi$ iniciando em $\varphi_{0}$, de modo que a sequência é da forma

$$
\left[\theta_{x}-\theta_{x+\delta \varphi}-\theta_{x+2 \delta \varphi}-\ldots-\theta_{x+\left(N_{f}-1\right) \delta \varphi}\right]
$$

A Fig. 2.8 apresenta o perfil de excitação das SSFPdx utilizando os incremento de fase 0 , $\pi / 2, \pi$ e $3 \pi / 2$. Note que o perfil é semelhante a SSFP de fase constante entretanto os pontos de máximos são deslocados no domínio da frequências por $\delta f$ de acordo com o incremento utilizado.
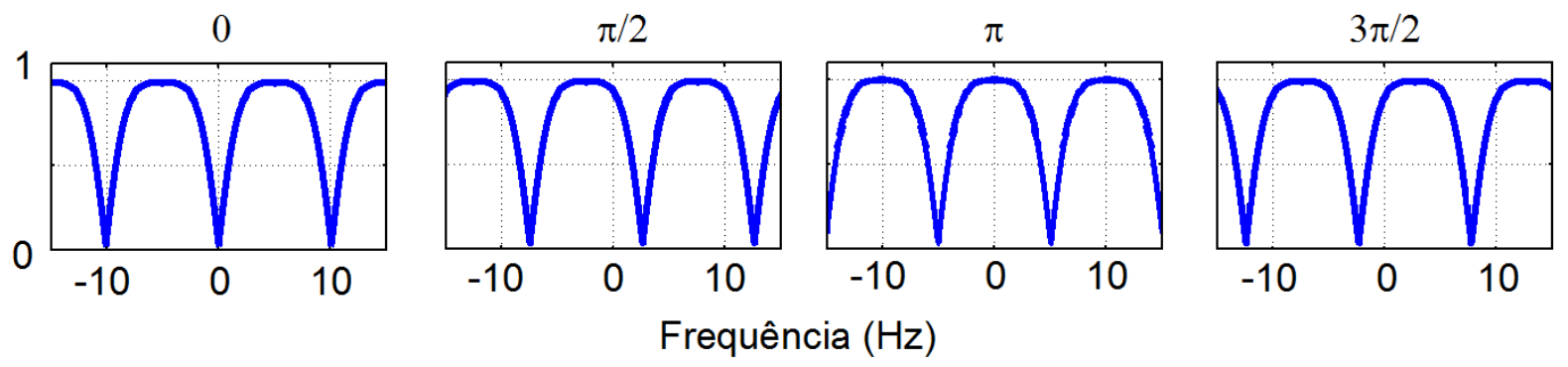

Figura 2.8- Perfil de excitação para as sequências SSFP com incremento de fase, $0, \pi / 2, \pi$ e $3 \pi / 2$, denominadas SSFPdx. Vale ressaltar que a SSFP com incremento de fase $\pi$ resulta na mesma sequência com alternância $(x,-x)$ SSFPx-x.

Fonte: Elaborada pelo autor.

A Fig. 2.9 apresenta sinais simulados no domínio do tempo quando aplicado sequências 
SSFPdx com incremento de fase $0, \pi / 4$ e $\pi$. Temos que quando o incremento é nulo, o pico presente na posição em ressonância é suprimido e assim no estado estacionário apresenta amplitude mínima. Na SSFPdx com $\pi / 4$ o perfil de excitação se desloca, logo a amplitude do sinal no estado estacionário é maior e para incremento de fase $\pi$ a amplitude do sinal torna-se máxima $\left(M_{0} / 2\right)$.

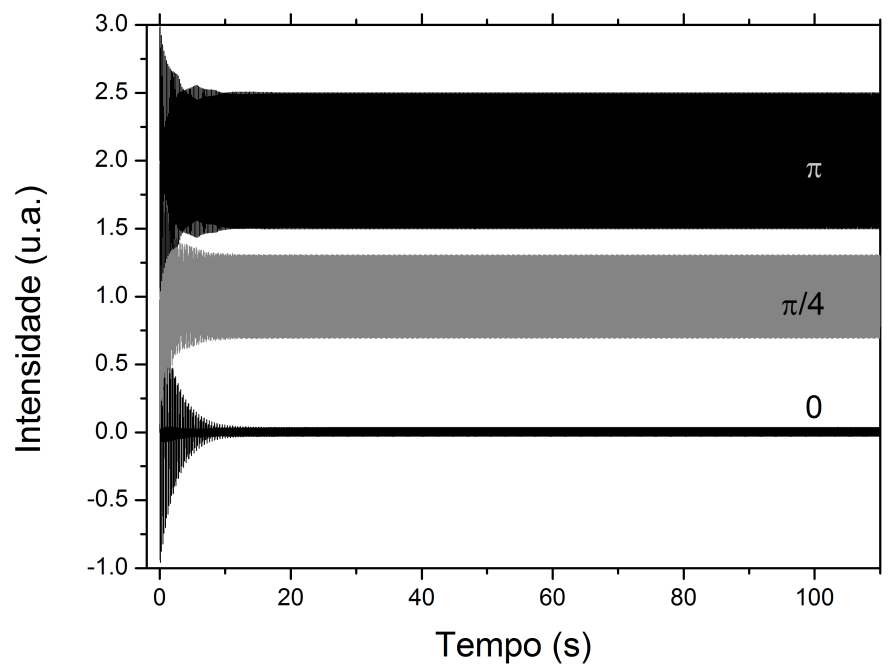

Figura 2.9- Sinais simulados da SSFPdx com incremento linear de fase. O incremento de fase define as posições de máxima e mínima amplitude no eixo de frequência, assim, na situação com um pico na posição em ressonância, o sinal é suprimido para incremento 0 , aumenta para $\pi / 4$ e torna-se máximo $\left(M_{0} / 2\right)$ para incremento de fase $\pi$.

Fonte: Elaborada pelo autor.

Diversas alternâncias de fase foram estudadas na busca de um perfil de excitação uniforme no eixo das frequências, o que eliminaria as regiões de supressão e anomalias de fase e amplitude observadas nos espectros das sequências SSFP convencional.

B. Hargreaves et al. demonstrou a eficiência na remoção de artefatos em $M R I$ utilizando a soma de múltiplas aquisições de SSFP com incremento de fase, tais como a soma dos dois experimentos de incremento 0 e $\pi$ da Fig. 2.8, ou dos quatro experimentos para obter maior precisão. ${ }^{55}$ Essa técnica foi pioneiramente proposta por Ernest ${ }^{29}$ em 1972 em contrapartida ao método QFT de deslocamento de offset proposto por A. Schwenk, porém não explorada na época.

Sequências SSFP com incremento linear de fase foram implementadas e desenvolvidas em experimentos de RMN de alta e baixa resolução e resultados e discussões são apresentados nos capítulos 4 e 5 . 


\subsection{Sinais assintóticos em estruturas periódicas}

Sequências de pulsos com estrutura periódica espaçados por tempos muito menores que os tempos de relaxação estabelecem um estado estacionário na magnetização. Nesta seção apresentamos uma forma geral para descrever sequências periódicas em campos magnéticos inomogêneos e apresentamos as principais características e semelhanças das sequências Continuous Wave Free Precession (CWFP), Split 180 e Driven-Equilibrium CPMG (DECPMG).

Devido a estrutura periódica dessas sequências é possível determinar uma matriz de rotação $\vec{R}(\hat{n}, \theta)$, com eixo $\hat{n}$ e ângulo de nutação $\theta$, que descreve a rotação total da magnetização no regime do estado estacionário durante sua evolução livre nos intervalos entre pulsos. A

Fig. 2.10 apresenta uma sequência de pulsos com estrutura periódica geral, onde $\vec{R}_{A}\left(\hat{n}_{A}, \theta_{A}\right)$ é a rotação total dos pulsos iniciais aplicados e $\vec{R}_{B}\left(\hat{n}_{B}, \theta\right)$ é o resultado de todas as rotações no intervalo periódico $T_{p}$, incluindo os tempos de precessão e pulsos.

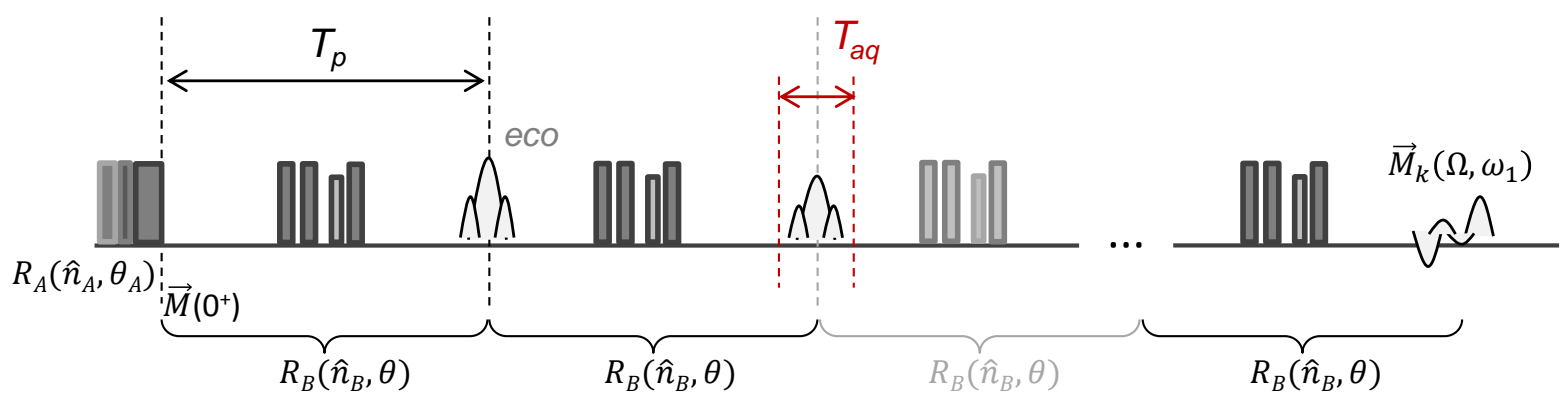

Figura 2.10- Esquema de uma sequência de pulsos periódica geral, onde $\vec{R}_{A}\left(\hat{n}_{A}, \theta_{A}\right)$ é a rotação total dos pulsos iniciais aplicados, que geram a magnetização $\vec{M}\left(0^{+}\right)$e $R_{B}\left(\hat{n}_{B}, \theta\right)$ é a matriz de rotação total do intervalo periódico $T_{p}$ que resulta da composição das rotações de precessão livre e dos pulsos.

Fonte: Elaborada pelo autor.

Aplicando $k$ períodos, temos

$$
\vec{M}_{k}=\left[R_{B}\left(\hat{n}_{B}, \theta\right)\right]^{k} R_{A}=R_{B}\left(\hat{n}_{B}, k \theta\right) R_{A}
$$

que pode ser reescrita como ${ }^{60,76}$

$$
\vec{M}_{k}=\hat{n}_{B}\left(\hat{n}_{B} R_{A}\right)+\left[R_{A}+\hat{n}_{B}\left(\hat{n}_{B} R_{A}\right) \hat{n}_{B}\right] \cos (k \theta)+\left[\hat{n}_{B} \times R_{A}\right] \operatorname{sen}(k \theta)
$$

A matriz $\vec{R}_{B}$, além dos demais parâmetros da sequência, é dependente do offset de frequên- 
cia local $\Omega$ e $\omega_{1}$ do pulso de RF. Na equação 2.5.2 temos que o primeiro termo não depende do número de ecos $k$, em contraste com o segundo e terceiro termos que oscilam com frequência $k \theta$. O sinal total é obtido com a contribuição de todas as componentes nas distribuições de frequências $\vec{M}_{k}\left(\Omega, \omega_{1}\right)$, de modo que em campos altamente inomogêneos e com a aplicação de muitos pulsos $k$, o segundo e terceiro termos da equação vão a zero. ${ }^{77}$ Nessa situação a magnetização para o $k$-ésimo eco é dada por

$$
\vec{M}_{k}=\exp \left(\frac{-k T_{p}}{T_{2, e f}}\right) \vec{R}(\hat{n}, k \theta) \vec{M}\left(0^{+}\right)
$$

onde $\vec{M}\left(0^{+}\right)$é a magnetização transversal logo após o primeiro pulso e $T_{2, \text { ef }}$ é o tempo efetivo do decaimento, que é dado por uma soma das médias das contribuições de $1 / T_{1}$ vezes o tempo que permanece ao longo da direção $\hat{z}$ e $1 / T_{2}$ vezes o tempo que permanece no plano $x y$

$$
\frac{1}{T_{2, e f}}=\frac{1}{T_{1}}\left\langle(\hat{z} \cdot \hat{n})^{2}\right\rangle+\frac{1}{T_{2}}\left(\left\langle(\hat{x} \cdot \hat{n})^{2}\right\rangle+\left\langle(\hat{y} \cdot \hat{n})^{2}\right\rangle\right)
$$

Para larga faixa de frequência de offset $\Omega, M$. Hurlimann mostrou numericamente que os coeficientes $\left\langle(\hat{z} . \hat{n})^{2}\right\rangle$ e $\left(\left\langle(\hat{x} . \hat{n})^{2}\right\rangle+\left\langle(\hat{y} . \hat{n})^{2}\right\rangle\right)$ estão na faixa entre 0,8-0,9 e 0,2-0,1 respectivamente. ${ }^{76}$

Essa descrição geral para blocos de pulsos periódicos em campos magnéticos inomogêneos, pode ser utilizada para descrever uma série de sequências de pulsos no estado estacionário, como a Split $180^{\circ 36,78}$, sequências CPMG-like com pulsos compostos ${ }^{65,77}$, CWFP e DECPMG. Nas próximas seções apresentamos as principais características dessas sequências.

\subsubsection{Continuous Wave Free Precession (CWFP)}

A aplicação periódica de pulsos iguais e espaçados sempre por $T_{p}$ gera um estado estacionário onde o sinal do eco e do FID se sobrepõem completamente, $\left|M_{x y}^{-}\right|=\left|M_{x y}^{+}\right|$. Este estado é obtido para tempos entre pulsos muito curtos, com $T_{p}<T_{2}^{*}$, onde $1 / T_{2}^{*}=1 / T_{2}+1 / T_{2, \text { inom }}$, onde $T_{2, \text { inom }}$ é a contribuição da inomogeneidade de campo. Nessas condições um estado estacionário em onda continua se forma, por isso denominado Continuous Wave Free Precession $\left(\right.$ CWFP). ${ }^{39}$ 
A Fig. 2.11 apresenta sinais simulados do comportamento da magnetização na transição para o estado CWFP, onde pulsos estão nas faixas cinzas e centrado em $t=0$, com a componente FID logo após o pulso ao lado direto de $t=0$ e a componente eco ao lado esquerdo do pulso. Nas simulações foram utilizados os valores de $T_{1}=150 \mathrm{~ms}, T_{2}=50 \mathrm{~ms}, T_{2}^{*}=0,5$ ms, $\theta=90^{\circ}$ e offset de frequência de $8,333 \mathrm{KHz}$ de (A até $\left.\mathrm{D}\right)$ e $6,666 \mathrm{KHz}$ em (E), para diferentes valores de $T_{p}$.

Na Fig. $2.11 \mathrm{~A}$ ) temos que para $T_{p}=5 T_{1}$ o tempo até o próximo pulso é longo o suficiente para a magnetização relaxar e retornar ao equilíbrio térmico, obtendo máximo de intensidade da magnetização e nenhuma componente eco no plano transversal logo antes do próximo pulso. Em B) $T_{p}=T_{2}$ a magnetização apresenta-se no regime SSFP inicial, onde a componente FID e eco são observadas entre os pulsos. A amplitude do FID é maior que do eco, porém menor que a amplitude do FID em A), pois $T_{p}<5 T_{1}$ não tendo retornado toda magnetização ao equilíbrio no momento do próximo pulso.

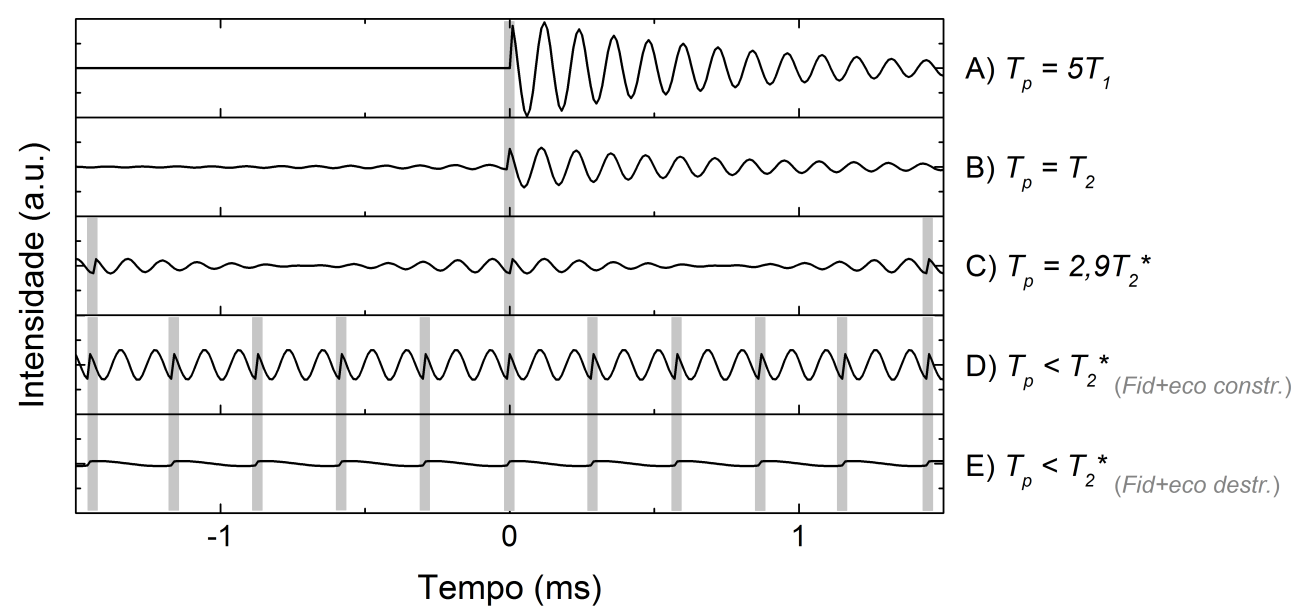

Figura 2.11- Sinais simulados com $T_{1}=150 \mathrm{~ms}, T_{2}=50 \mathrm{~ms}, T_{2}^{*}=0,5 \mathrm{~ms}$ para os valores de tempo entre pulsos: A) $T_{p}=5 T_{1}$, B) $T_{p}=T_{2}$, C) $T_{p}=2,9 T_{2}^{*}$, D) and E) $T_{p}<T_{2}^{*}$. O offset de frequência é $8,333 \mathrm{KHz}(\mathrm{A}$ até $\mathrm{D})$ e $6,666 \mathrm{KHz}$ em E).

Fonte: Elaborada pelo autor.

As Fig. 2.11 C) até E) apresentam mais de um período entre pulsos no intervalo de $-1,5$ e 1,5 ms. C) apresenta dois períodos pois $T_{p}=2,9 T_{2}^{*}$, onde vemos que o FID e eco tem amplitudes similares. Note que a componente FID nesse caso decai mais rápido que $T_{2}^{*}$ em A) e B). Isso resulta da interação parcialmente destrutiva entre o FID e eco na região central do sinal SSFP.

Os sinais das Fig. 2.11 D) e E) apresentam $T_{p}=0,3 \mathrm{~ms}<T_{2}^{*}$. Nessa condição a 
componentes FID e ecos estão totalmente sobrepostas, com amplitude fortemente dependente do offset de frequência ou do ângulo de precessão $\Phi=\Omega T_{p}$, gerando um regime especial da SSFP conhecido como Continuous Wave Free Precession.

Pela equação 2.2 .12 vemos que a amplitude do estado estacionário é modulado em função de $\Phi=\Omega T_{p}$ e na condição $\Omega T_{p}=2 n \pi$ a magnetização se anula, pois $|M| \rightarrow 0$. Isso ocorre porque a componente FID e eco estão defasadas de $180^{\circ}$, resultando numa interferência destrutiva. Para o caso do offset estabelecer $\Phi=(2 n+1) \pi$, o FID e eco encontram-se em fase e assim se observa um máximo de intensidade do sinal, dado por

$$
\left|M_{s s}\right|=\frac{M_{0}}{1+T_{1} / T_{2}}
$$

mostrando que a amplitude é no máximo metade da amplitude $M_{0}$ quando $T_{1} / T_{2}=1$.

Na Fig. 2.11 D) $\Phi=5 \pi$, offset de frequência de 8,333 KHz, gerando interferência construtiva do FID e eco e portanto amplitude máxima do sinal CWFP. Fig. 2.11 E) $\Phi=4 \pi$, offset de frequência de $6,666 \mathrm{KHz}$, com interferência destrutiva entre as componentes que resulta no mínimo de amplitude do sinal.

Assim temos que magnitude do sinal CWFP é dependente da razão $T_{1} / T_{2}$ e como $T_{p}<T_{2}^{*}$, centenas de sinais CWFP podem ser promediados em um período de tempo $T_{1}$, aumentando a razão s/r em mais de uma ordem de magnitude em experimentos FID ou Spin-Eco com mesmo tempo de duração. Detalhes para realizar medida simultânea dos tempos de relaxação com o método CWFP são apresentados a seguir.

\section{Medidas simultâneas de $T_{1}$ e $T_{2}$ pela CWFP}

O primeiro método baseado na SSFP para medida dos tempos de relaxação $T_{1}$ e $T_{2}$ foi proposto por A. Schwenk e J. Kronenbitter ${ }^{79}$ em 1977. O método baseava-se na realização de dois experimentos. No primeiro obtém-se a razão $T_{1} / T_{2}$ através da medida da amplitude do sinal SSFP em função do ângulo do pulso $\theta$. No segundo passo obtém-se a soma $\left(T_{1}+T_{2}\right)$ através do tempo $T^{*}$ que é o tempo de decaimento do sinal até atingir o estado estacionário. Através desses dois experimentos determina-se os valores de $T_{1}$ e $T_{2}$.

Em 2005 T. Venâncio et al. ${ }^{39}$ adaptaram o método propondo uma técnica para realizar 
medidas simultâneas de $T_{1}$ e $T_{2}$ através da CWFP em um único experimento. $\mathrm{O}$ método é baseado no tempo característico que a magnetização leva para estabelecer o estado estacionário $T^{*}$, calculado na seção 2.3 , fixando os parâmetros $\theta=90^{\circ}$ e $\Phi=(2 n+1) \pi$. Pela equação 2.3.17, temos que o tempo característico é dado por

$$
T^{*}=\frac{2 T_{1} T_{2}}{T_{1}+T_{2}}
$$

Rearranjando as equações 2.5 .5 e 2.5 .6 temos

$$
T_{1}=\frac{T^{*} / 2}{\left|M_{s s}\right| / M_{0}} \quad ; \quad T_{2}=\frac{T^{*} / 2}{1-\left|M_{s s}\right| / M_{0}}
$$

Deste modo, determinando $T^{*},\left|M_{s s}\right|$ e $M_{0}$ podemos obtemos $T_{1}$ e $T_{2}$ em um único experimento. A Fig. 2.12 apresenta sinais simulados da evolução da magnetização desde o primeiro pulso até a estabilização do estado estacionário. Em A) apresentamos a CWFP $\left[\frac{\pi}{2}-T_{p}\right]_{n}$ e em B) a CP-CWFP $\pi-\frac{T_{p}}{2}\left[\frac{\pi}{2}-T_{p}\right]_{n}$, supondo $T_{1}=150 \mathrm{~ms}, T_{2}=50 \mathrm{~ms}, T_{p}=$ $0,3 \mathrm{~ms}$ e $T_{2}^{*}=20 \mathrm{~ms}$.

A região em cinza escuro representa o transiente inicial de alternância de amplitude entre os pulsos pares e ímpares governado pelo decaimento com $T_{2}^{*}$. A região cinza claro se estabelece com o fim das oscilações de amplitude formando um estado quase-estacionário que decai com a constante de tempo $T^{*}$ até estabelecer o estado estacionário na região branca, onde o módulo da magnetização permanece em $\left|M_{s s}\right|$ enquanto for continuada a aplicação dos pulsos.

A sequência CP-CWFP foi proposta posteriormente ${ }^{47}$ a fim de ampliar a região dinâmica de $T^{*}$ dos experimentos CWFP quando $T_{1} \approx T_{2}$. No início da sequência de pulso se acrescenta um pulso de $\pi$ e após um tempo $T_{p} / 2$ inicia-se o bloco CWFP com pulsos de $\pi / 2$ espaçados por $T_{p}$. Nessas condições os sinais obtidos para amostras com $T_{1} \approx T_{2}$ apresentam maior amplitude na região dinâmica, melhorando a razão s/r na determinação de $T^{*}$, Fig. 2.12 B). Em situações com $T_{1}>T_{2}$ a CWFP fornece região dinâmica maior.

Na seção 4 discutiremos os recentes avanços nos métodos CWFP com a introdução de alternância de fase e o estudo da distribuição de tempos de relaxação na CWFP. 

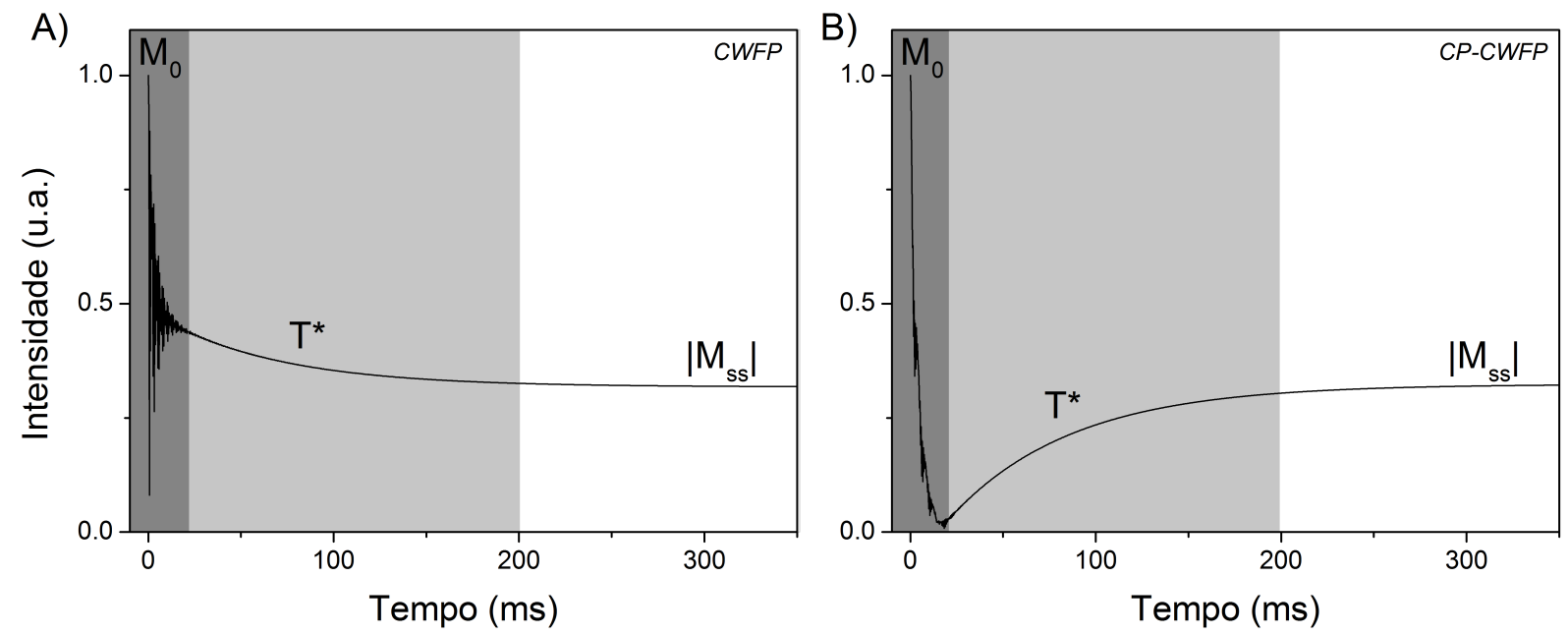

Figura 2.12- Magnitude dos sinais simulados para sequência de pulsos A) CWFP $\left[\pi / 2-T_{p}\right]_{n}$ e B) CP-CWFP $\pi-\frac{T_{p}}{2}\left[\pi / 2-T_{p}\right]_{n}$. Através dos parâmetros $M_{0},\left|M_{s s}\right|$ e $T^{*}$ obtém-se em uma única aquisição os tempos de relaxação $T_{1}$ e $T_{2}$.

Fonte: Elaborada pelo autor.

\subsubsection{SPLIT $180^{\circ}$}

A primeira descrição da sequência Split $180^{\circ}$ foi feita em 2003 na patente (US 6580272 B2), ${ }^{78}$ baseados nos desenvolvimentos iniciais de D. Freed ${ }^{80}$ com o grupo de cientistas associados a companhia Schlumberger-Doll Research. Essa técnica foi desenvolvida para extração de informações das rochas petrolíferas como porosidade, difusibilidade e volume de fluído preso em sistemas com campos magnéticos altamente inomogêneos em medidas de perfil well-logging no setor petrolífero. Posteriormente foi publicado o artigo $^{36}$ e a conferência ${ }^{81}$ que descrevem mais propriedades da sequência.

A sequência consiste na aplicação de $\frac{\pi}{2}-\frac{\tau_{1}}{2}\left[\frac{\pi}{2}-\tau_{2}-\frac{\pi}{2}-\tau_{1}\right]_{n}$, onde a aquisição do ecos ocorrem no intervalo $\tau_{1}$, muito semelhante a uma CPMG, onde o pulso de $\pi$ do ciclo foi dividido em dois pulsos de $\pi / 2$ espaçados pelo tempo $\tau_{2}$, com $\tau_{2}<\tau_{1}<<T_{1}$ e $T_{2}$, Fig. 2.13.

A Split $180^{\circ}$ gera dois sinais simultâneos. O primeiro é um decaimento denominado CPMGlike, com tempo de relaxação $T_{2, e f}$ (efetivo) e o segundo um sinal que cresce até um estado estacionário com amplitude dependente da razão $T_{1} / T_{2}$. A Fig. 2.14 apresenta os sinais característicos da sequência Split $180^{\circ}$ extraída da Patente (US 6580272 B2). ${ }^{78}$ Em A) observase o sinal CPMG-like (410) e o sinal que cresce até o estado estacionário (420). Em B) e C) são apresentados o perfil dos ecos formados no decaimento CPMG-like (initial shape) e no estado estacionário (long time shape) respectivamente. 


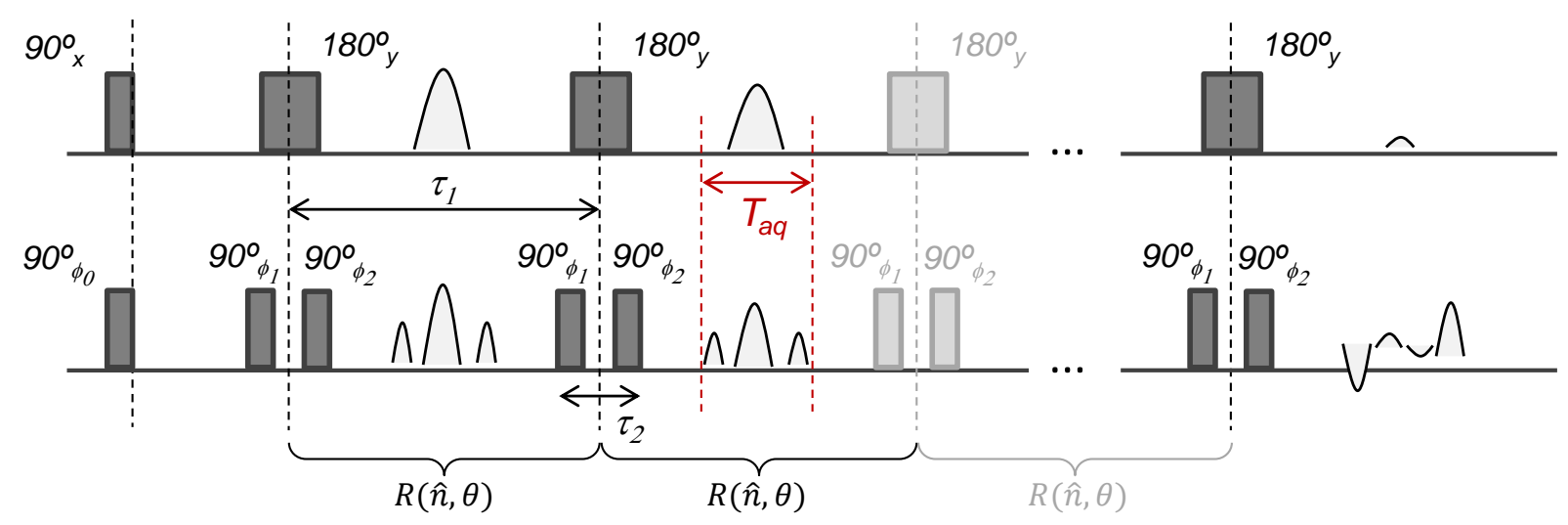

Figura 2.13- Acima sequência de pulsos CPMG e abaixo Split $180^{\circ}$. Na Split $180^{\circ}$ dois pulsos de $90^{\circ}$ são espaçados por $\tau_{2}$. As fases associadas aos pulsos são $\phi_{0}, \phi_{1}$ e $\phi_{2}$, que para $\phi_{1}=\phi_{2}$ temos a Split $180^{\circ}$ Simétrica e para $\phi_{1}=-\phi_{2}$ Anti-simétrica.

Fonte: Elaborada pelo autor.
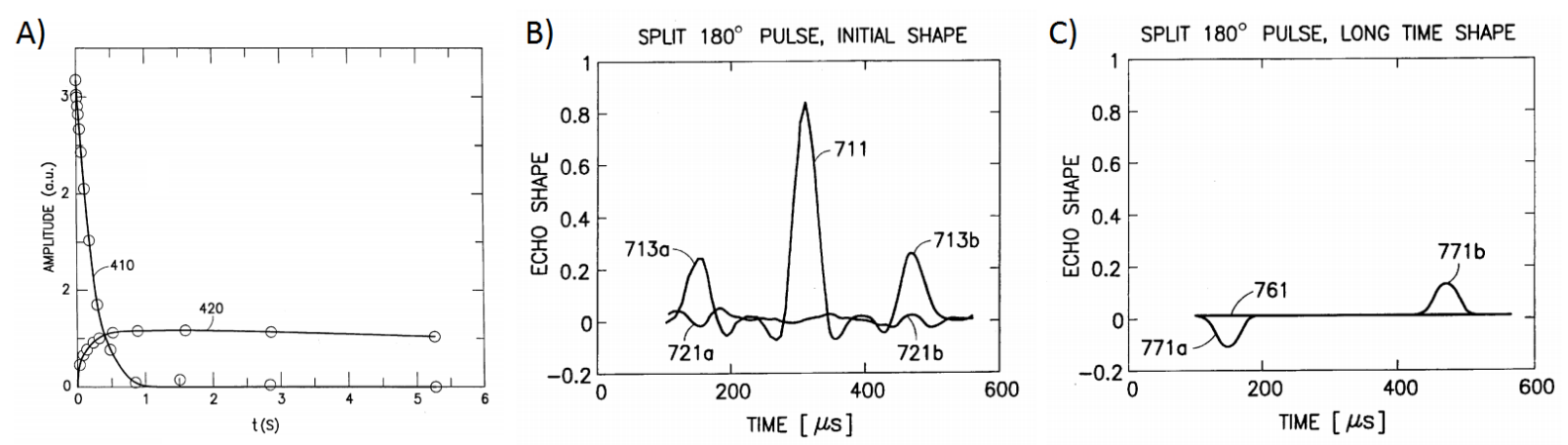

Figura 2.14- Sinais da sequência de pulsos Split $180^{\circ}$. Em A) observa-se o sinal CPMG-like (410) e o sinal que cresce até o estado estacionário (420). Em B) e C) são apresentados o perfil dos ecos formados no decaimento CPMG-like e no estado estacionário respectivamente.

Fonte: Adapatada de FREED; HURLIMANN; SCHEVEN. ${ }^{78}$

Utilizando a descrição de sequências de pulsos periódicas, na Fig. 2.13 vemos que a sequência Split $180^{\circ}$ apresenta a região periódica denotada por $R(\hat{n}, \theta)$, que pode ser decomposta em três matriz de precessão $P_{0}, P_{1}$ e $P_{2}$, com intervalos de precessão livre $\Delta t_{0}, \Delta t_{1}$ e $\Delta t_{2}$ e duas matrizes de pulsos $R_{1}$ e $R_{2}$.

No limite de aplicação rápida $\tau_{1}<<T_{1}, T_{2}$, temos

$$
M(t)=M_{t}(t)+M_{s s}(t)
$$

onde $M_{t}(t)$ é a magnetização transiente do sinal CPMG-like e $M_{s s}(t)$ a magnetização que vai ao estado estacionário. A determinação dessas componentes podem ser exploradas através de matrizes de rotação similar ao descrito na seção 2.2 ou através da descrição por caminhos de coerência. 
Na referência ${ }^{78}$ é mostrado que

$$
M_{t}(t)=\exp \left(-\frac{A t}{T_{1}}-\frac{B t}{T_{2}}\right)
$$

e

$$
M_{s s}=\frac{M_{0}}{A+B\left(T_{1} / T_{2}\right)}(\hat{n} \cdot \hat{\rho}) \hat{n}
$$

onde $\hat{n}$ é o eixo de rotação, $\vec{\rho}=\sum_{0}^{k}\left(\prod P_{i} R_{i}\right) a_{j} \hat{z}$ e $a_{j}=\Delta t_{j} / \Delta t$, com

$$
A=\sum_{j=0}^{k} a_{j}\left[\left(\hat{x} \prod_{i=1}^{j}\left(P_{i} R_{i}\right) P_{0} \hat{n}\right)^{2}+\left(\hat{y} \prod_{i=1}^{j}\left(P_{i} R_{i}\right) P_{0} \hat{n}\right)^{2}\right]
$$

e

$$
B=\sum_{j=0}^{k} a_{j}\left(\hat{z} \prod_{i=1}^{j}\left(P_{i} R_{i}\right) P_{0} \hat{n}\right)^{2}
$$

sendo $P_{i}$ e $R_{i}$ as matrizes de precessão e de rotação dos pulsos, de acordo com a seção 2.1.

Nas referências ${ }^{36,78,81}$ mais detalhes sobre os efeitos das fases escolhidas para os pulsos $\phi_{0}, \phi_{1}$ e $\phi_{2}$ são descritos, além de resultados ainda não publicados por M. Hürlimann et al. na geração dos perfis Butterfly. ${ }^{81} \mathrm{Na}$ seção 4.2 apresentamos nossa implementação dessa sequência de pulsos em um magneto unilateral - Single Sided NMR-MOUSE - apresentando aplicações práticas e estudos dos padrões Butterfly.

\subsubsection{Driven-Equilibrium CPMG}

A sequência de pulsos Driven-Equilibrium Carr-Purcell Meiboom-Gill (DECPMG) tem por objetivo determinar de forma rápida a média das razões dos tempos de relaxação $T_{1} / T_{2}$ em função de $T_{2}$, gerando um mapa de correlação $T_{1} / T_{2}-T_{2}$ ou o mapa $T_{1}-T_{2}$. Isso é feito através de dois experimentos de uma dimensão, sendo o primeiro uma CPMG convencional e o segundo o sinal adquirido com a sequência DECPMG.

A técnica padrão para obtenção dos mapas de correlação $T_{1}-T_{2}$ é através do experimentos bi-dimensional $T_{1}-T_{2}$, introduzidos por Y.Q. Song et al. em $2002^{82-84}$, onde a sequência $\pi-\tau_{1(i)}-\frac{\pi}{2}-\frac{\tau}{2}-[\pi-\tau]_{n}$ é aplicada para uma lista com vários valores para $\tau_{1}(i)$. Através de uma transformada de Laplace bi-dimensional (2D), se obtêm o mapa da distribuição $T_{1}-T_{2}$, 
como explicado no esquema da Fig. 2.15.

A)

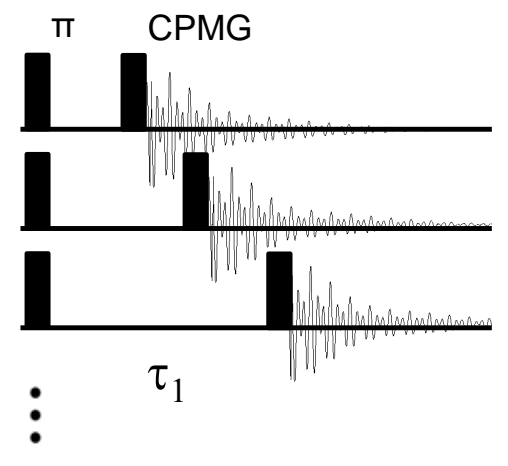

B)

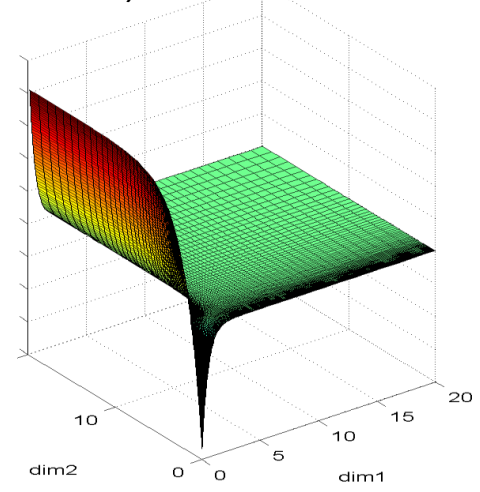

C)

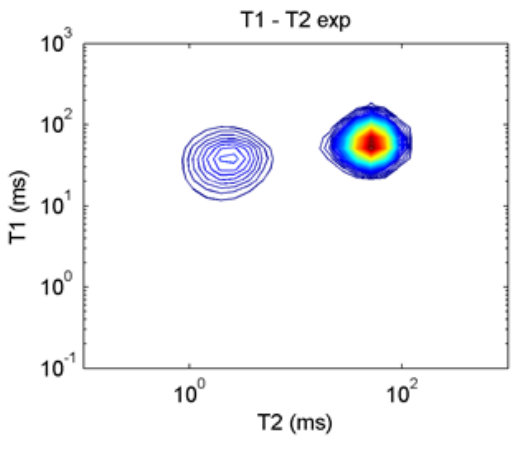

Figura 2.15- Experimento bi-dimensional de correlação $T_{1}-T_{2}$. Em A) é apresentada a sequência de pulsos $\pi-\tau_{1}(i)-\frac{\pi}{2}-\frac{\tau}{2}-[\pi-\tau]_{n}$, onde $\tau_{1}(i)$ é uma lista de valores com $i$ experimentos na dimensão

1. B) apresenta o perfil característico dos sinais adquiridos com tal sequência de pulsos e em C) após processamento do sinal com a Transformada Inversa de Laplace bi-dimensional o mapa de distribuição $T_{1}-T_{2}$ obtido.

Fonte: Elaborada pelo autor.

A sequência de pulsos DECPMG foi inicialmente apresentada em 2002, na patente (US 6597171 B2 $)^{85}$ e posteriormente no artigo. ${ }^{35} \mathrm{~A}$ ideia é aplicar inicialmente um bloco de pulsos Driven Equilibrium (DE) formado por um pulso inicial de $90^{\circ}$, seguido por um pulso de $180^{\circ}$ que refocaliza a magnetização no centro do intervalo $\tau_{2}$, seguido por outro pulso de $180^{\circ}$ que refocaliza novamente a magnetização e sofre um pulso de $90^{\circ}$ exatamente na posição da formação do segundo eco, ver Fig 2.16.

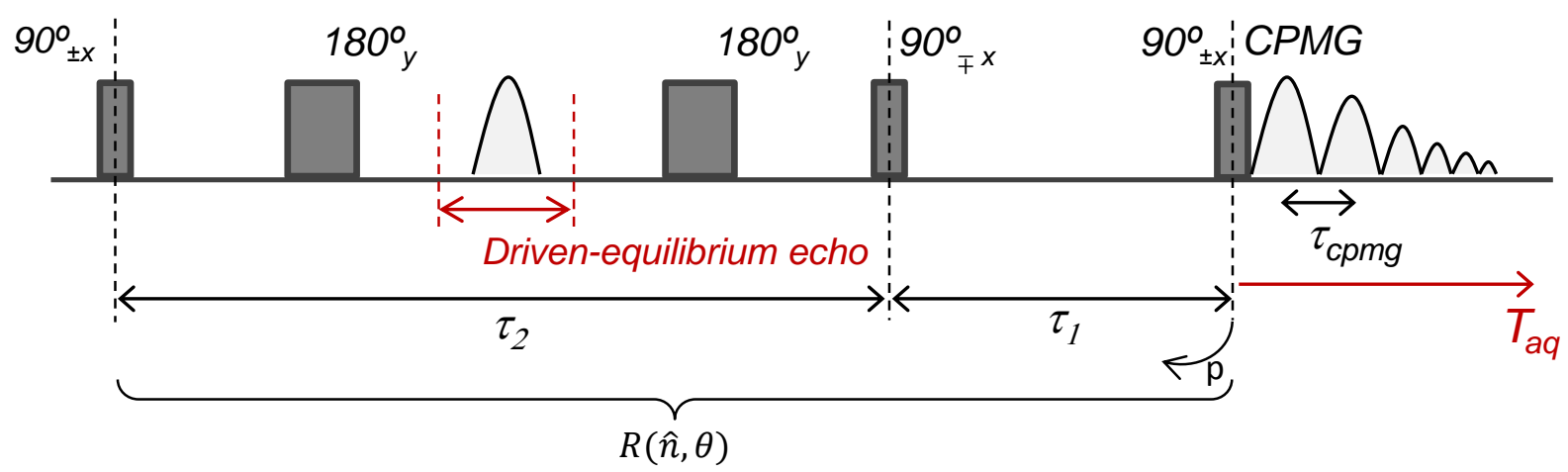

Figura 2.16- Sequência de pulsos Driven-Equilibrium Carr-Purcell Meiboom-Gill DECPMG.

Fonte: Elaborada pelo autor.

Após o último pulso de $90^{\circ}$ do bloco DE, um intervalo de tempo $\tau_{1}$ é esperado e esse bloco é repetido $p$ vezes. Após um tempo transiente a magnetização entra num estado estacionário, 
onde a amplitude é dada por

$$
M_{d e}=M_{0} \frac{1}{1+\frac{\tau_{2} T_{1}}{\tau_{1} T_{2}}}
$$

Atingido esse estado estacionário, imediatamente a sequência de pulsos realiza uma aquisição CPMG-like gerando um sinal que decai a partir da amplitude definida pelo bloco DE e com constante de tempo $T_{2, e f}$, com

$$
M_{c}\left(q 2 t_{c p m g}\right)=M_{0} \exp \left\{\frac{-q 2 t_{c p m g}}{T_{2, e f}}\right\}
$$

Uma vez realizado ambos os experimentos CPMG e DECPMG, o processamento dos sinais é executado através da Transformada Inversa de Laplace unidimensional como descrito na referência ${ }^{35}$, baseado na relação entre a amplitude dos sinais

$$
\frac{T_{1}}{T_{2}}=\frac{\tau_{1}}{\tau_{2}}\left(\frac{M_{c}}{M_{d e}}-1\right)
$$

Além da DECPMG, novas modificações desse sequência foram recentemente introduzidas na literatura por F. Deng et al. ${ }^{86}$ e estendidas para determinação de viscosidade em fluídos. ${ }^{87}$

Na seção 4.2 apresentamos resultados obtidos com a implementação da DECPMG no magneto compacto mini-halbach, comparando mapas $T_{1}-T_{2}$ obtidos pela técnica com as sequência convencional. 


\section{Capítulo 3}

\section{Materiais e Métodos}

\subsection{Espectrômetros e magnetos}

\subsubsection{Alta resolução}

Os espectros de ${ }^{1} \mathrm{H},{ }^{13} \mathrm{C}$ e ${ }^{15} \mathrm{~N}$ de alta resolução apresentados nessa tese foram adquiridos no espectrômetro Avance III Bruker 14,1 Tesla, com frequência de ressonância do hidrogênio em $600 \mathrm{MHz}$, localizado na Embrapa Instrumentação, São Carlos-SP. Na metade inicial do desenvolvimento desse projeto foi também utilizado o espectrômetro Inova da Varian 9,4 T (400 MHz). A Fig. 3.1 apresenta uma foto do espectrômetro da Bruker nas instalações do laboratório da Embrapa Instrumentação.

\subsubsection{Baixa resolução}

Ao longo do desenvolvimento do projeto foi possível trabalhar em diversos espectrômetros de bancada de RMN de baixa resolução. Na Embrapa Instrumentação foram utilizados os equipamentos da Spinlock Magnetic Resonance Solution (Cordoba, Argentina) SLK-100, SLK200 e SLK-1399, com campo magnético de 0,25 T, 0,26 T e 0,23 T, com frequências de ressonância do hidrogênio próximas de $10 \mathrm{MHz}$ e respectivamente abertura de bore de $30 \mathrm{~mm}$ para a série SLK-100 e 200 e 12 cm para o SLK-1399. Também foi utilizado um espectrômetro Minispec mq20 (Bruker Analytic GmbH, Rheinstetten, Alemanha), com tubos de $10 \mathrm{~mm}$ e controle de temperatura. 


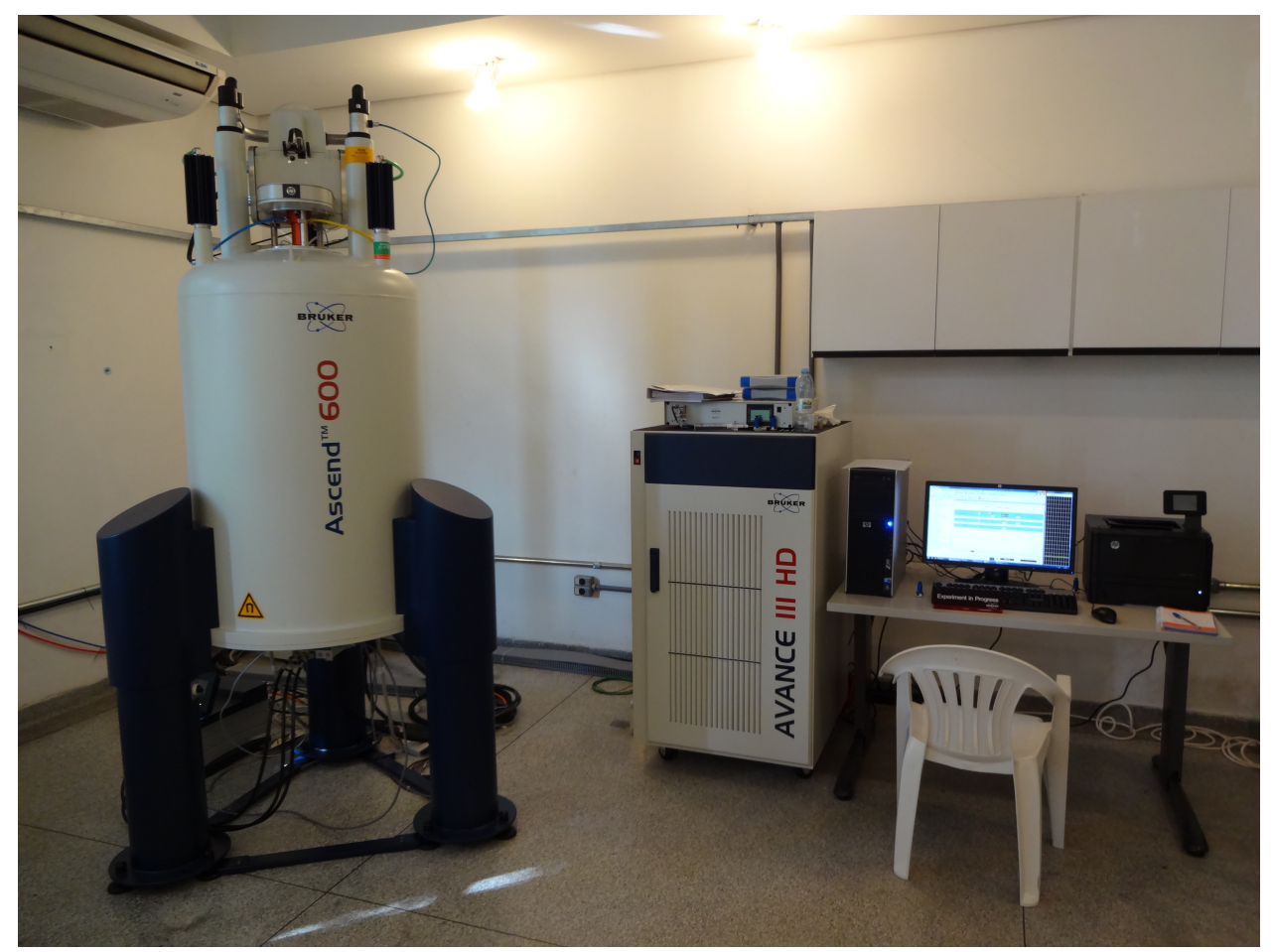

Figura 3.1- Espectrômetro Avance III da Bruker de 14,1 T com frequência de ressonância de $600 \mathrm{MHz}$ para hidrogênio com sonda multinuclear de $5 \mathrm{~mm}$ de detecção inversa, localizado na Embrapa Instrumentação, São Carlos-SP.

Fonte: Elaborada pelo autor.
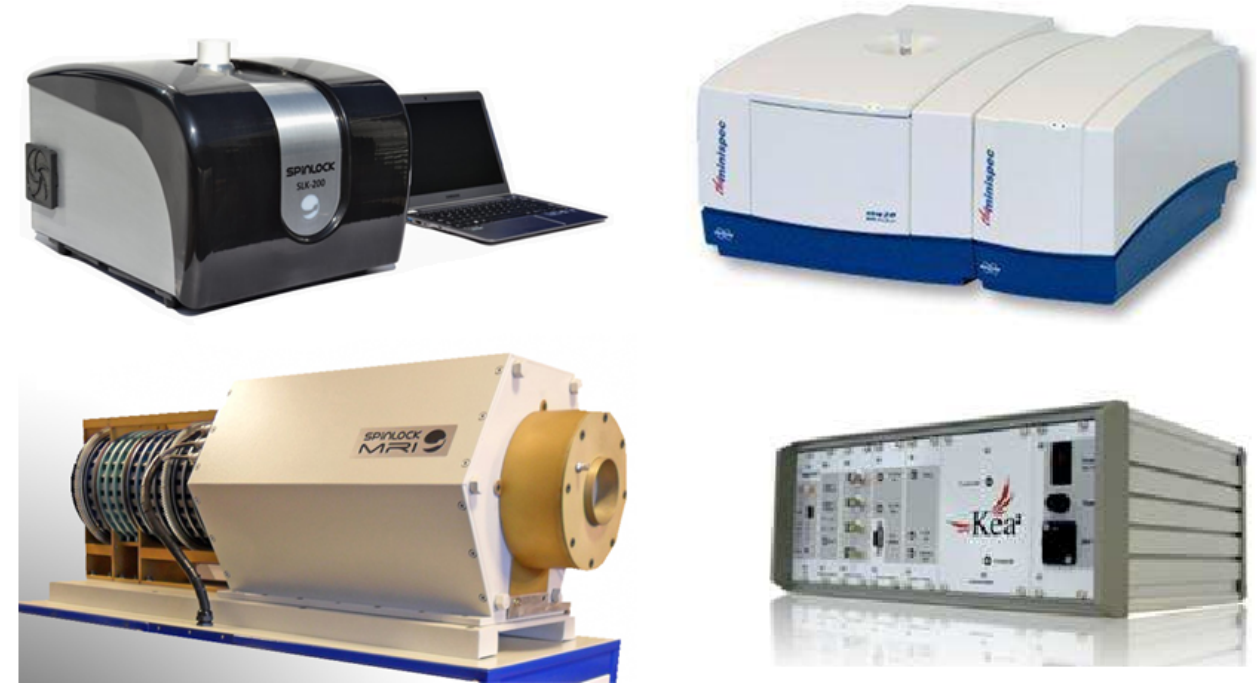

Figura 3.2- Espectrômetros de baixa resolução. Na esquerda acima SLK-200 e abaixo SLK-1399, ambos da SpinLock. Na direita acima Minispec mq20 da Bruker e abaixo Kea da Magritek.

Fonte: Elaborada pelo autor.

Na etapa desenvolvida durante o estágio de pesquisa no exterior (Aachen, Alemanha), foram utilizados espectrômetros KEA da Magritek (Wellington, Nova Zelândia) com sistemas magnéticos mini-halbach e MOUSE (Mobile Universal Surface Explorer) construídos pela ACT GmbH (Aachen, Alemanha), como descrito na seção 4.2. 


\subsection{Preparo de amostras}

\subsubsection{Seção 5 - SSFP}

Os espectros de alta resolução apresentados na seção 5 foram adquiridos no espectrômetro da Bruker em tubos de $5 \mathrm{~mm}$ a $25^{\circ} \mathrm{C}$. Para os experimentos de ${ }^{1} \mathrm{H}$ preparamos as soluções:

- $20 \mu \mathrm{l} \mathrm{H}_{2} \mathrm{O}$ em $500 \mu \mathrm{l} \mathrm{D}_{2} \mathrm{O}$;

- $20 \mu \mathrm{l} \mathrm{H}_{2} \mathrm{O}$ e $20 \mu \mathrm{l}$ de acetona $\left(\mathrm{C}_{3} \mathrm{H}_{6} O\right)$ em $500 \mu \mathrm{l} \mathrm{D}_{2} O$;

Para os espectros de ${ }^{13} C$, as soluções:

- 3,0 mg de sacarose $\left(\mathrm{C}_{12} \mathrm{H}_{22} \mathrm{O}_{11}\right)$ dissolvida em $500 \mu \mathrm{l} \mathrm{D}_{2} \mathrm{O}$;

- $105,0 \mathrm{mg}$ de sacarose dissolvida em $500 \mu \mathrm{l} \mathrm{D}_{2} \mathrm{O}$;

- 0,18 mg de Brucina $\left(\mathrm{C}_{23} \mathrm{H}_{6} \mathrm{~N}_{2} \mathrm{O}_{4}\right)$ dissolvida em $500 \mu \mathrm{l}$ dimetilsulfóxido DMSO - $d_{6}$; E para os espectros de ${ }^{15} N$ :

- Formamida $\left(\mathrm{CH}_{3} \mathrm{NO}\right)$ em dimetilsulfóxido $\mathrm{DMSO}-d_{6}$;

- $200 \mu \mathrm{l}$ de anilina $\left(C_{6} H_{7} N\right)$ e $200 \mu \mathrm{l}$ de trietilamina $\left(C_{6} H_{15} N\right)$ em $200 \mu \mathrm{l}$ acetronitrila deuterada $\left(C D_{3} C N\right)$;

O deslocamento químico é expresso em $\delta$, ppm - partes por milhão, relativo ao tretametilsilano (TMS).

\subsubsection{Seção $6.2-K B D M$}

Os experimentos apresentados na seção 6.2 foram adquiridos com a sequência de pulsos CarlPurcell-Melbourne-Gill (CPMG). ${ }^{15,17}$ Foram utilizadas três intensidades de campo magnético, 0,047, 0,26 e 0,47 Tesla, correspondendo a frequência de ressonância do hidrogênio de 2,11 e $20 \mathrm{MHz}$. O imã permanente de 0,26 T é controlado pelo espectrômetro SLK-200 pertencente a Embrapa Instrumentação, São Carlos. Os magnetos de 0,047 e 0,47 T foram controlados pelo TECMAG LapNMR console localizados no Instituto de Física de São Carlos, USP. 
Amostras padrões - phanthons - foram criadas com soluções de água destilada dopadas com $\mathrm{CuSO}_{4} .5 \mathrm{H}_{2} \mathrm{O}$ em diferentes concentrações de modo a estabelecer padrões numa faixa de tempos de relaxação longitudinal $T_{2}$ entre 0,01 e 3 segundos. A amostra S1 possui concentração $12 \mathrm{mg} / \mathrm{ml}, \mathrm{S} 26 \mathrm{mg} / \mathrm{ml}, \mathrm{S} 33 \mathrm{mg} / \mathrm{ml}$, diminuindo sempre próximo a metade da concentração anterior até a amostra S11, com $0,012 \mathrm{mg} / \mathrm{ml}$. O phanthon S12 é composto por água destilada pura. Cada solução foi colocada em tubos eppendorf com volume de 1,5 ml. Os phanthons (S1-S12) foram medidos no SLK-200 em $11 \mathrm{MHz}$ na frequência do hidrogênio. O número de ecos foi otimizado para cada amostra e utilizado $\tau$ entre 100 e $500 \mu s$, com aquisição usando alternância de fase cyclops.

Utilizou-se também uma cerâmica de alumina porosa, sintetizada e analisada por E.T. Montrazi do IFSC-USP, de acordo com o descrito na referência. ${ }^{88}$ Essa cerâmica foi caracterizada por porosimetria de intrusão de mercúrio (MIP) e microtomografia de raio-X $(\mu \mathrm{CT})$, e é utilizada no estudo de meios porosos por RMN em baixa resolução. Neste trabalho, essa cerâmica foi saturada com solução de $\mathrm{NaCl}$ e utilizada como um meio poroso padrão bem conhecido para comparação dos métodos de Inversa de Laplace e KBDM. No final da seção 6.2, são apresentados resultados obtidos com grãos, óleo lubrificante e as rochas porosas do setor petrolífero Sillurian, Indiana e Combfield. A cerâmica sintética foi medida em $20 \mathrm{MHz}$, com tempo ao eco de $200 \mu s$ com 2, 8, 32 e 128 médias. As rochas porosas Sillurian, Indiana e Combfield foram medidas em $2 \mathrm{MHz}$ com tempo ao eco de $300 \mu \mathrm{s}$ e 32 médias.

Para comparação com o método proposto KBDM da seção 6.2, os sinais de CPMG foram invertidos com a Transformada Inversa de Laplace utilizando o método NNLS-ILT (non-negative least squares inverse Laplace transform) usando regularização de Tikhonov e SVD. ${ }^{89}$

\subsection{Programação das sequências de pulsos e simula- ções numéricas}

As sequências de pulsos foram implementadas nas respectivas linguagens de programação dos softwares de controle dos espectrômetros acima descritos. Isso englobou os softwares TopSpin 3.2, Condor-ID e Prospa 3.2. As sequências de pulsos implementadas incluem as SSFP e 
CWFP com alternância e incremento de fase, a Split $180^{\circ}$ e DECPMG, que podem ser obtidas diretamente com o autor.

As simulações numéricas desenvolvidas para as sequências de pulsos foram implementadas em um pacote de funções no Matlab (MATrix LABoratory) com o método de somatória de isocromatas em uma distribuição lorentziana de componentes. O método é baseado no descrito nas referências ${ }^{55,73}$ e publicamos um artigo de ensino na revista Química Nova ${ }^{66}$, onde é fornecido os códigos e instruções básicas.

\subsection{Processamento de dados}

Os espectros em alta resolução foram processados nos respectivos softwares dos espectrômetros, utilizando funções de apodização Traf e Transformada de Fourier. Os espectros KBDM foram processados na implementação em linguagem $C$ no ambiente Code Builder do software OriginPro-8.5 (OriginLab Corporation) desenvolvido por C.J. Magon. ${ }^{90}$

Os sinais de baixa resolução foram processados para obtenção de tempos característicos dos decaimentos CPMG, IR e CWFP, onde os principais softwares utilizados foram o OriginPro-8.5 e pacotes de fitting exponencial no Matlab.

Para a Transformada Inversa de Laplace foram desenvolvido funções em Matlab utilizando o método Non-negative Least Squares Inverse Laplace transform (NNLS-ILT) com regularização de Tikhonov e decomposição em valores singulares (SVD), de acordo com as referências. ${ }^{89,91}$ Esses métodos são descritos no capítulo 6 e os códigos desenvolvidos são apresentados nos apêndices 8. Versões atualizadas dos códigos podem ser obtidas diretamente com o autor.*

\footnotetext{
* Os códigos das sequências de pulsos e pacotes de processamento de inversão de laplace podem ser obtidos diretamente com o autor: tiagobuemoraes@gmail.com
} 



\section{Capítulo 4}

\section{Estado Estacionário em Baixa Resolução}

A determinação da distribuição dos tempos de relaxação $T_{1}$ e $T_{2}$ fornece informações importantes sobre as propriedades físico-química dos materiais, tais como, ambiente molecular, mobilidade e viscosidade.

A importância da relaxometria de RMN vem crescendo nos últimos anos com aplicações em diversas áreas, como no setor industrial para análises de rotina em materiais porosos, polímeros, petróleo e produtos alimentícios. Por se tratar de uma técnica não destrutiva e de baixo custo, o potencial de aplicações é muito grande e novos métodos rápidos e robustos tornam-se cada vez mais desejáveis.

Recentemente, vários métodos baseados em sequências de pulsos no estado estacionário foram propostos para realização de medidas rápidas dos tempos de relaxação ou da razão $T_{1} / T_{2}$. Essas sequências de pulsos apresentam blocos de pulsos periódicos, separados por tempos entre pulsos muito menores que os tempos de relaxação $T_{p}<<T_{1}, T_{2}$, como nos métodos CWFP ${ }^{39}$, DECPMG ${ }^{35}$ e Split $180^{\circ} .{ }^{36}$ Nas seções seguintes apresentamos estudos relacionados com essas sequências de pulsos, sendo que parte deste trabalho foi desenvolvido em um intercâmbio BEPE-FAPESP (Proc. 2014/16952-3) no instituto ITMC na RWTH Aachen University (Rheinisch-Westfälische Technische Hochschule Aachen), na Alemanha, sob orientação do Prof. Dr. Bernhard Bluemich e do Dr. Ernesto Danieli. 


\subsection{Continuous Wave Free Precession}

Dentre os recentes avanços da técnica de precessão livre em onda contínua (CWFP) uma contribuição significativa foi realizada com a introdução de esquemas de alternância de fases nas sequências, que possibilitaram a eliminação da dependência com o deslocamento da frequência de offset e do valor do tempo entre pulsos $T_{p}$, descrito no artigo. ${ }^{34}$

Um segundo desafio explorado nesses últimos anos foi a análise da distribuição dos tempos de relaxação nas sequências de pulsos CWFP. Apesar do método CWFP ser aplicado na determinação rápida e simultânea dos tempos de relaxação $T_{1}$ e $T_{2}$, essa técnica é mais apropriada na análise de amostras com decaimento mono-exponenciais. Desenvolver uma técnica CWFP para determinação rápida e simultânea das distribuição dos tempos de relaxação $T_{1}$ e $T_{2}$ multiexponenciais não parece ser trivial, porém essa busca resultou no desenvolvimento da sequência de pulsos 'CWFP- $T_{1}$ ', para determinação rápida dos tempos de relaxação longitudinal, como descrito a seguir. ${ }^{92}$

\subsubsection{CWFP com alternância de fase}

Uma característica das sequências CWFP e CP-CWFP é a necessidade do deslocamento da frequência de offset para um valor $2 \pi f=(2 n+1) \pi$, com $n$ inteiro, como descrito na seção 2.5.1. Nessas condições as equações 2.5 .6 e 2.5.7 são válidas e o método consegue determinar de maneira precisa e simultânea os tempos de relaxação para amostras monoexponencial.

Uma série de simulações e experimentos foram realizadas introduzindo diversos esquemas de alternância de fase nas sequências CWFP e CP-CWFP. Esses estudos mostraram que a introdução de um incremento de fase constante $\Delta \varphi$ nos pulsos e receptor, resulta no deslocamento da frequência de offset por $\Omega^{\prime}=\Omega+\frac{\Delta \varphi}{T_{p}}$. Os máximos de intensidade são obtidos com $\Phi=(2 n+1) \pi$, assim os máximos na CWFP com incremento de fase são obtidos quando

$$
\Omega T_{p}+\Delta \varphi=(2 n+1) \pi
$$

A equação 4.1.1 mostra que os pontos de máximo do perfil de excitação são deslocados no 
eixo da frequência de acordo com o incremento de fase introduzido. Desse modo, ao utilizar um incremento de fase $\pi$, alternância $(x,-x)$ por exemplo, obtemos um máximo de intensidade da posição central da janela, ou seja em $f_{0}=0$ offset de frequência nulo, tornando assim a CWFP uma técnica on-resonance. Além de colocar o máximo de amplitude em ressonância, essa implementação promove para o método uma independência dos tempos entre pulsos $T_{p}$, como descrito na Fig. 4.1.

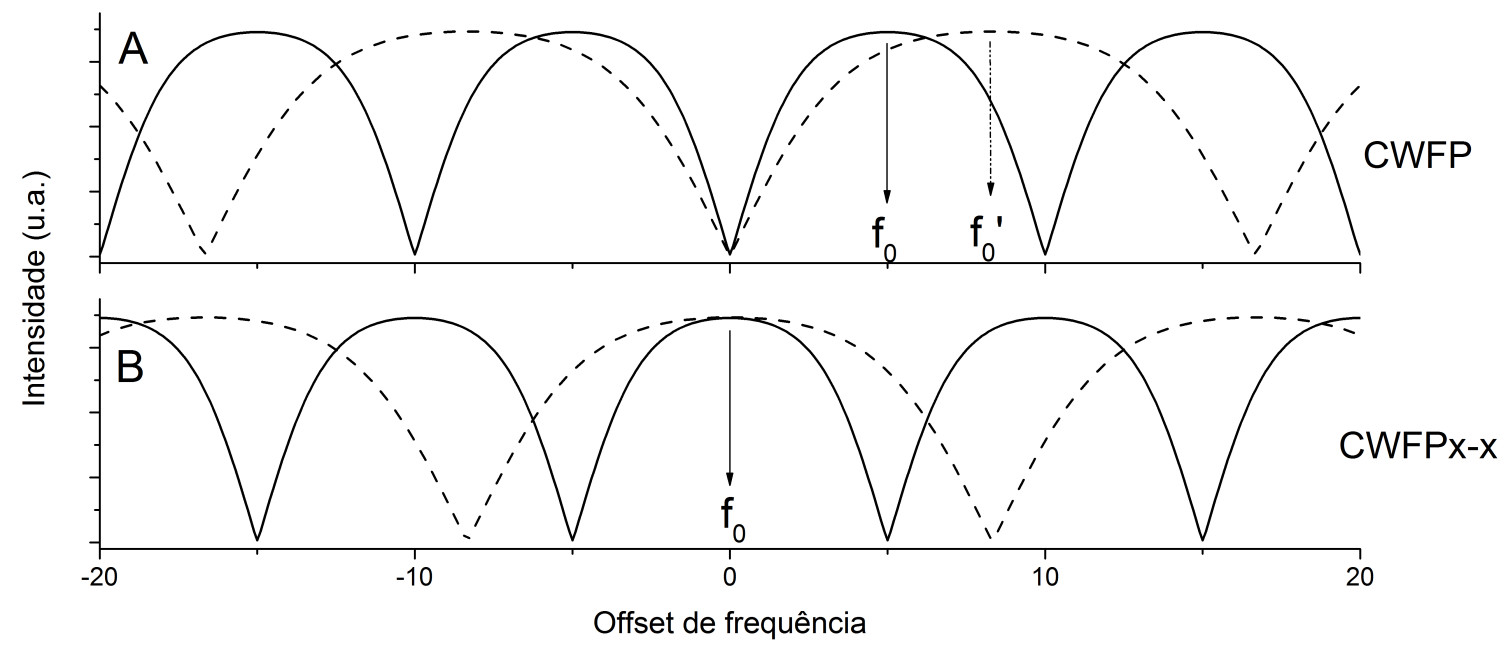

Figura 4.1- Sinais CWFP simulados em A) sem alternância de fase e B) com alternância de $\pi$. Utilizando um incremento de fase $\varphi=\pi$ ( $\mathrm{x},-\mathrm{x}$ por exemplo) o perfil é deslocado de modo que os máximos de intensidade ficam presentes nos pontos de mínimo de intensidade da CWFP sem alternância de fase. Linhas contínuas é para $T_{p}=100 \mu \mathrm{s}$ e tracejada $60 \mu \mathrm{s}$.

Fonte: Elaborada pelo autor.

Para a CWFP sem alternância de fase e com $T_{p}=100 \mu$ s, linha contínua na Fig. 4.1 A), o primeiro máximo de amplitude do perfil é observado em $f_{0}=5 \mathrm{~Hz}$. Se alterarmos o valor do tempo entre pulsos para $T_{p}=60 \mu \mathrm{s}$, linha tracejada, os máximos do perfil se deslocam e a nova posição do primeiro máximo fica em $f_{0}^{\prime}=8,3 \mathrm{~Hz}$.

Na Fig. 4.1 B) destacamos o comportamento da CWFP com alternância de fase $\pi$. Nesse caso, a posição de máximo de amplitude se encontra no centro da janela espectral e alterando o valor de $T_{p}$ entre $100 \mu$ s (linha contínua) ou $60 \mu$ s (linha tracejada), em ambas as situações o máximo de amplitude está em $f_{0}=0 \mathrm{~Hz}$, não havendo necessidade de deslocamento de offset.

A sequência CP-CWFP apresenta comportamento do perfil de excitação similar ao descrito acima com a introdução de alternância $(x,-x)$ ou $(y,-y)$. A vantagem da CP-CWFP se estabelece no pulso CP que maximiza a amplitude da região dinâmica das sequências, melhorando a 
relação sinal ruído na determinação do $T^{*}$.

A Fig. 4.2 apresenta uma comparação resumida de sinais simulados para as sequências CWFP, CP-CWFP, CWFPx-x, CP-CWFPx-x nas situações em ressonância e fora de ressonância. Foram considerados nas simulações $T_{1}=100 \mathrm{~ms}, T_{2}=50 \mathrm{~ms}, T_{p}=300 \mu \mathrm{s}, T_{2}^{*}=20$ $\mu \mathrm{S}$.
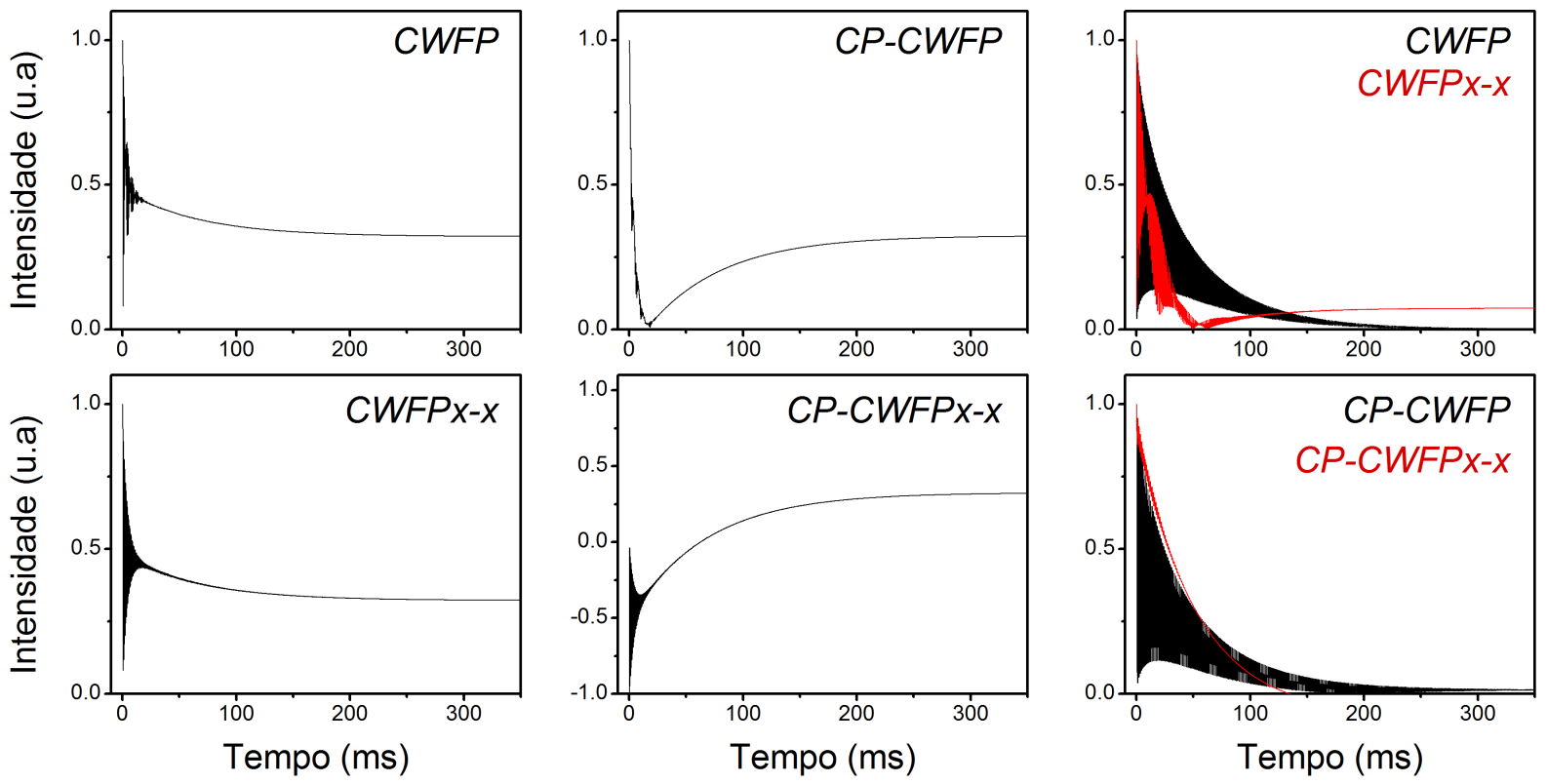

Figura 4.2- Sinais simulados das sequências de pulsos CWFP, CP-CWFP, CWFPx-x e CP-CWFPx-x com $T_{1}$ $=100 \mathrm{~ms}, T_{2}=50 \mathrm{~ms}, T_{p}=300 \mu \mathrm{s}, T_{2}^{*}=20 \mu \mathrm{s}$.

Fonte: Elaborada pelo autor.

Na primeira coluna à esquerda vemos que a CWFP e CWFPx-x apresentam sinais muito semelhantes obtendo os mesmos resultados de tempos de relaxação, ambas na condição $\Phi=$ $(2 n+1) \pi$. Na segunda coluna da Fig. 4.2, a cima temos o sinal CP-CWFP que é característico por apresentar maior região dinâmica que a CWFP quando $T_{1} \approx T_{2}$, crescendo ao estado estacionário desde amplitude zero até $\left|M_{s s}\right|$. Na segunda coluna abaixo, o sinal CP-CWFPx-x evidência seu ganho de intensidade na região dinâmica, obtendo a melhor razão $s / r$ entre as sequências do tipo CWFP, onde a magnetização inicia sua transição ao estado estacionário desde amplitudes negativas até positivas.

Na terceira coluna da Fig. 4.2 são apresentados os sinais das sequências fora da condição $\Phi=(2 n+1) \pi$. Para a CWFP e CP-CWFP foram colocados o offset de frequência em ressonância, resultando nos sinais de cor preta na figura. As sequências com alternância de fase CWFPx-x e CP-CWFPx-x foram adquiridas com o offset de frequência em $\Phi=2 \pi$, em verme- 
Iho. Esses resultados mostram que nessas condições a componente FID e eco se sobrepõem destrutivamente não formando o estado estacionário em onda contínua da magnetização e decaindo a zero com uma constante de tempo diferente de $T^{*}$.

Nas referências ${ }^{34}$ e na dissertação ${ }^{93}$ foram publicados os detalhes das técnicas CWFP com alternância de fase, apresentando comparações com os tempos de relaxação obtidos por CPMG, IR e CWFP convencional em uma série de análises experimentais e simulações.

\subsubsection{CWFP para análise multi-exponencial}

O método CWFP se propõe a determinar de maneira rápida e simultânea os tempos de relaxação $T_{1}$ e $T_{2}$, entretanto essa técnica é mais apropriada na análise de amostras com componentes mono-exponenciais. A extensão do método para análise de sinais bi-tri ou multiexponenciais tornaria o método muito útil na determinação rápida das distribuição de tempos de relaxação em amostras complexas.

Na tese de doutorado do A.A. Souza ${ }^{94}$, foram realizados alguns estudos comparando o tempo $T^{*}$ obtido na CWFP e CP-CWFP em sistemas multi-exponenciais com a média geométrica dos tempos de relaxação em experimentos bi-dimensionais $T_{1}-T_{2}$. A proposta de estimar a média geométrica dos tempos de relaxação com o tempo $T^{*}$ resultante da CWFP se mostrou promissora em aplicações no setor petrolífero, porém uma demonstração mais completa das hipóteses ficaram por ser apresentadas.

A Fig. 4.3 esquematiza a complexidade da determinação da distribuição dos tempos de relaxação pelas sequências do tipo CWFP. Os sinais apresentados foram adquiridos com a CP-CWFP de dois eppendorfs de $1,5 \mathrm{ml}$ com água dopada com cobre, um com concentração de $3,0 \mathrm{~g} / \mathrm{l}$ e outro com $0,47 \mathrm{~g} / \mathrm{l}$ resultando em valores de tempo de relaxação $T_{2}=63 \mathrm{~ms}$ e $T_{2}=1,14 \mathrm{~s}$ respectivamente. As curvas CP-CWFP para a amostra 1 é apresentada em Fig. 4.3 A) e para a amostra 2 em Fig. 4.3 B). A curva da Fig. 4.3 C) foi obtida através da aquisição do sinal quando os dois eppendorfs foram introduzidos e adquiridos simultaneamente no magneto.

As respectivas transformadas inversa de laplace dos sinais são apresentadas nas Fig. 4.3 $A^{\prime}$ ) até $C^{\prime}$ ). Esses resultados, dentre outros omitidos aqui, mostram que quando submetidos 
a experimentos com mais de uma componente exponencial, o comportamento da CP-CWFP e da CWFP é apresentar a distribuição de todos os tempos $T^{*}$ do conjunto.
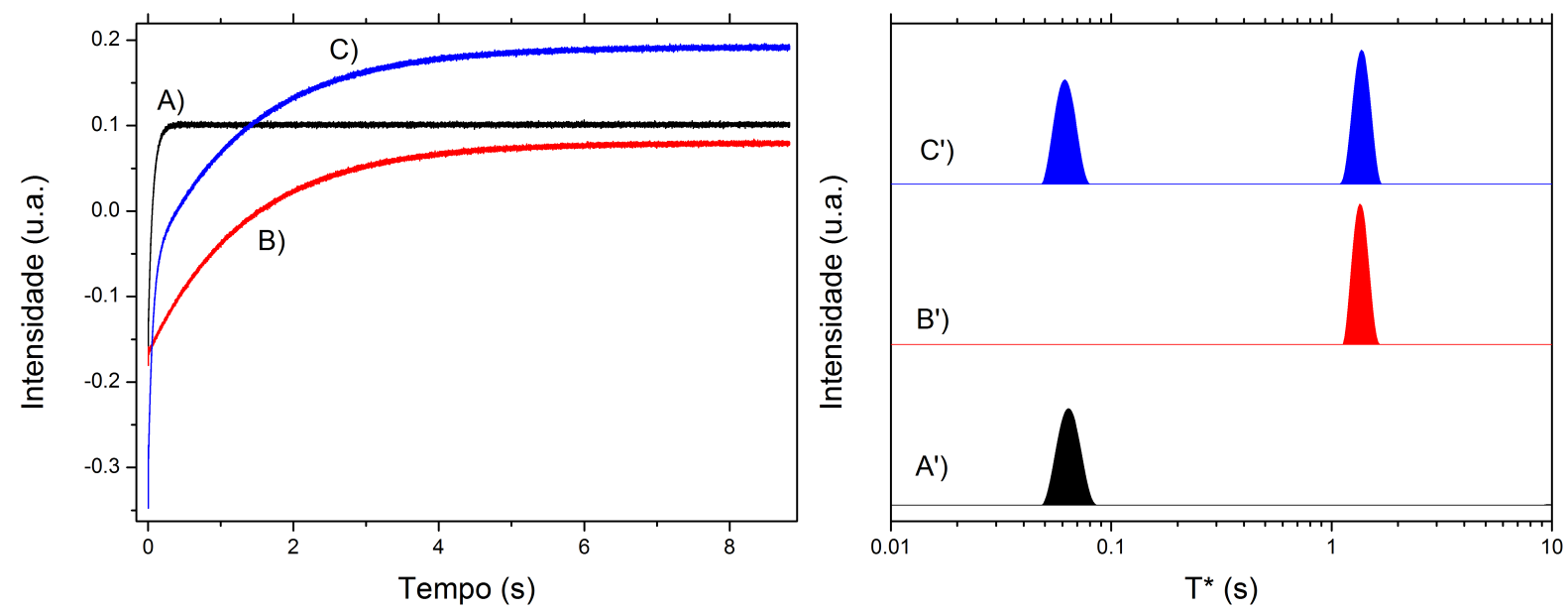

Figura 4.3- $\mathrm{A}$ esquerda sinais CP-CWFP adquiridos de amostras de água dopada com cobre, em $\mathrm{A}$ ) com concentração de $3,0 \mathrm{~g} / \mathrm{l}$, em B) com $0,47 \mathrm{~g} / \mathrm{l}$ e em C) aquisição simultânea dos dois eppendorfs. A direita são apresentadas as respectivas Transformada inversa de laplace dos sinais, apresentando os tempos característicos encontrados.

Fonte: Elaborada pelo autor.

O problema dessa análise se estabelece na posterior determinação dos tempos de relaxação $T_{1}$ e $T_{2}$ a partir do fitting bi-exponencial da curva Fig. $4.3 \mathrm{C}$ ) ou pela distribuição Fig. $4.3 \mathrm{C}^{\prime}$ ). Sabemos que a determinação no caso mono-exponencial é realizada com as equações $T_{1}=$ $\frac{T^{*} / 2}{\left|M_{s s}\right| / M_{0}}$ e $T_{2}=\frac{T^{*} / 2}{1-\left|M_{s s}\right| / M_{0}}$, entretanto $\left|M_{s s}\right|$ na curva Fig. $\left.4.3 \mathrm{C}\right)$ é $\left|M_{s s}\right|=\left|M_{s s}\right|_{1}+\left|M_{s s}\right|_{2}$, ou seja, a soma da contribuição das duas componentes. Além do clássico problema mal-posto da inversão de soma de exponenciais, nessa situação, a soma das amplitudes $\left|M_{s s}\right|$ também admite múltiplas soluções, evidenciando um obstáculo no desenvolvimento de uma versão da CWFP para determinação rápida das distribuições de tempos de relaxação $T_{1}$ e $T_{2}$ em amostras multi-exponenciais.

Apesar da dificuldade encontrada na tentativa de determinação simultânea da distribuição dos tempos de relaxação, esses estudos nos levou ao desenvolvimento de uma ferramenta útil na determinação rápida da distribuição dos tempos de relaxação $T_{1}$, com a técnica $C W F P-T_{1} \cdot{ }^{92}$ 


\section{CWFP- $T_{1}$}

Na seção 2.3 mostramos que o tempo característico dos sinais CWFP é dependente do ângulo do pulso $\theta$ utilizado, conforme a equação

$$
T^{*}=\frac{2 T_{1} T_{2}}{T_{1}(1-\cos \theta)+T_{2}(1+\cos \theta)}
$$

Na CWFP são utilizados pulsos de $\theta=90^{\circ}$, de modo que $T^{*}$ e $\left|M_{s s}\right|$ são dependentes da razão $T_{1} / T_{2}$ e da magnitude $M_{0}$, onde $T^{*}=2 T_{1} T_{2} /\left(T_{1}+T_{2}\right)$ e $\left|M_{s s}\right|=M_{0} T_{2} /\left(T_{1}+T_{2}\right)$. Note que o tempo característico $T^{*}$ na CWFP se estabelece na faixa entre $2 T_{2}$ e $T_{1}$, respectivamente para as condições limites em $T_{1}>T_{2}$ e $T_{1}=T_{2}$.

A equação 4.1.2 também mostra que $T^{*}$ pode ir de $T_{2}$ até $T_{1}$, independente do valor da razão $T_{1} / T_{2}$, quando o ângulo dos pulsos variam de $180^{\circ}$ até ângulos próximos de zero. A Fig. 4.4 apresenta resultados experimentais (símbolos sólidos) e calculados pela equação 4.1.2 (linha sólida) de $T^{*}$ para solução aquosa de $1,0.10^{-2}$ mol. $L^{-1} \mathrm{CuSO}_{4}$ com símbolo $(O)$ e 2, $5 \cdot 10^{-4} \mathrm{~mol} \cdot \mathrm{L}^{-1} \mathrm{MnSO}_{4}(\triangle)$ em função do ângulo do pulso.

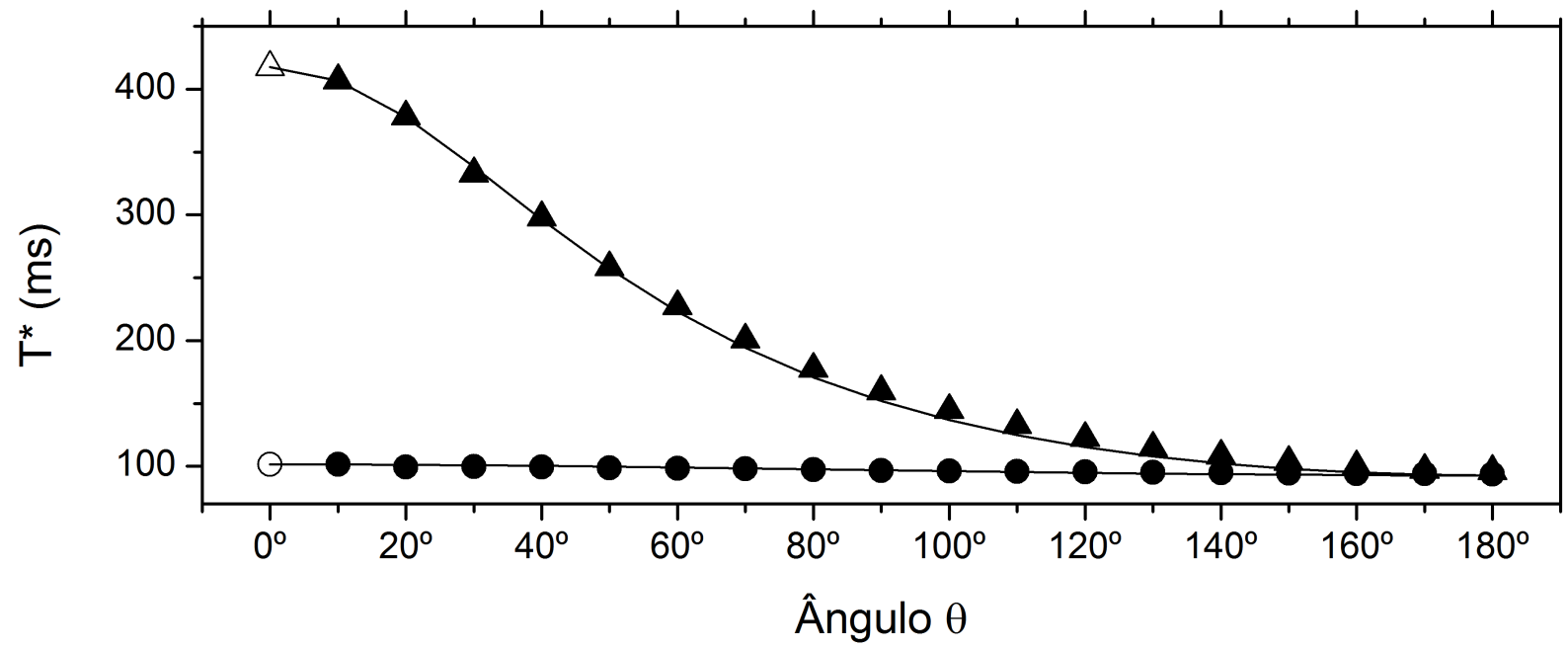

Figura 4.4- Variação do tempo característico $T^{*}$ da sequência CP-CWFPx-x em função do ângulo $\theta$ para soluções aquosas de $\mathrm{CuSO}_{4}$ e $\mathrm{MnSO}_{4}$, usando $T_{p}=300 \mu$ s. As linhas sólidas foram calculadas pela equação 4.1.2. Os símbolos $(O)$ e $(\triangle)$ com preenchimento branco são os valores de $T_{1}$ obtidos com a sequência de pulsos IR.

Fonte: Elaborada pelo autor.

A Fig. 4.4 mostra que conforme se reduz o ângulo $\theta$ das sequências tipo CWFP, no caso foi utilizada a CP-CWFPx-x porém em todas esse efeito é observado, o tempo $T^{*}$ vai de $T_{2}$ em $180^{\circ}$ até muito próximo de $T_{1}$ para ângulos pequenos. Os símbolos $(O)$ e $(\triangle)$ com 
preenchimento branco são os valores de $T_{1}$ obtidos com a sequência de pulsos IR. A ideia fundamental do método CWFP- $T_{1}$ é otimizar essa estimativa de $T_{1}$ através da medida de $T^{*}$ com as sequências CWFP, realizando uma estimativa rápida do tempo de relaxação longitudinal em apenas uma aquisição.

Como vimos na seção $4.1 .1^{34}$, simulações e experimentos mostraram que a CP-CWFPx-x é a sequência que apresenta a maior região dinâmica obtendo melhor razão $s / r$ entre as técnicas CWFP. Assim a sequência aqui denominada CWFP- $T_{1}$ é da forma $180^{\circ}-T_{p} / 2-\left[\theta_{ \pm \varphi}-T_{p}-\right]_{n}$, onde os ângulos $\theta$ do bloco de pulsos é pequeno, tipicamente $10^{\circ}$ ou $5^{\circ}$.

$\mathrm{O}$ método proposto tem algumas similaridades com os métodos de Look e Locker ${ }^{95}$ e Kaptein et al. ${ }^{96}$ para medidas rápidas de $T_{1}$ em alta resolução. O método de Kaptein é baseado na sequência de pulsos $180^{\circ}-T_{p} / 2-\left[\theta-T_{p}-\right]_{n}$, onde $\theta$ é o ângulo dos pulsos, tipicamente $30^{\circ}$. Pulsos homospoil eram utilizados após cada aquisição para destruir a magnetização transversal e reduzir a formação de ecos. Eles mostraram que o método obtém estimativa de $T_{1}$ mais rápido que os métodos convencionais por Inversão Recuperação ou Saturação Recuperação. ${ }^{97}$ Os tempos entre pulsos do método são da ordem de segundos e correções numéricas do tempo estimado são necessárias, através das equações $1 / T_{1}^{*}=1 / T_{1}+1 / T_{\theta}$, onde $T_{\theta}=-\theta / \ln (\cos \theta) .{ }^{96}$

O método CWFP- $T_{1}$ usa uma sequências de pulsos semelhante, com um pulso inicial de $180^{\circ}$, seguido de um trem CWFP com alternância de fase $x$ e -x e pulsos com ângulos pequenos, tipicamente $10^{\circ}$ ou $5^{\circ}$, sem utilização de pulsos homospoil, uma vez que o sinal é adquirido na condição de onda contínua (continuous wave free precession) com a imposição de FID e eco construtivos com $\Phi=(2 n+1) \pi$ e resultante do tempo muito curto entre pulsos, $T_{p}$ na ordem de $300 \mu \mathrm{s}$.

A Fig. 4.5 A) e B) apresentam sinais simulados e experimentais da sequência CWFP- $T_{1}$ em função do pulso $\theta$ aplicado. Em A) sinais simulados considerando $T_{1}=100 \mathrm{~ms}, T_{2}=$ $50 \mathrm{~ms}, T_{2}^{*}=900 \mu \mathrm{s}, T_{p}=300 \mu \mathrm{s}$ e em B) sinais experimentais da solução de $\mathrm{MnSO}_{4}$, onde os índices de i) até vi) são respectivamente para os ângulos de $90^{\circ}, 60^{\circ}, 45^{\circ}, 30^{\circ}, 20^{\circ}$, $10^{\circ}$ e $5^{\circ}$. Esses resultados mostram que quando reduzimos o ângulo dos pulsos da sequência a amplitude da região dinâmica diminui consideravelmente, indo de $0,5 M_{0} \mathrm{em} \theta=90^{\circ}$ até 
aproximadamente $0,05 M_{0}$ em $\theta=5^{\circ}$ reduzindo por um fator 10 .
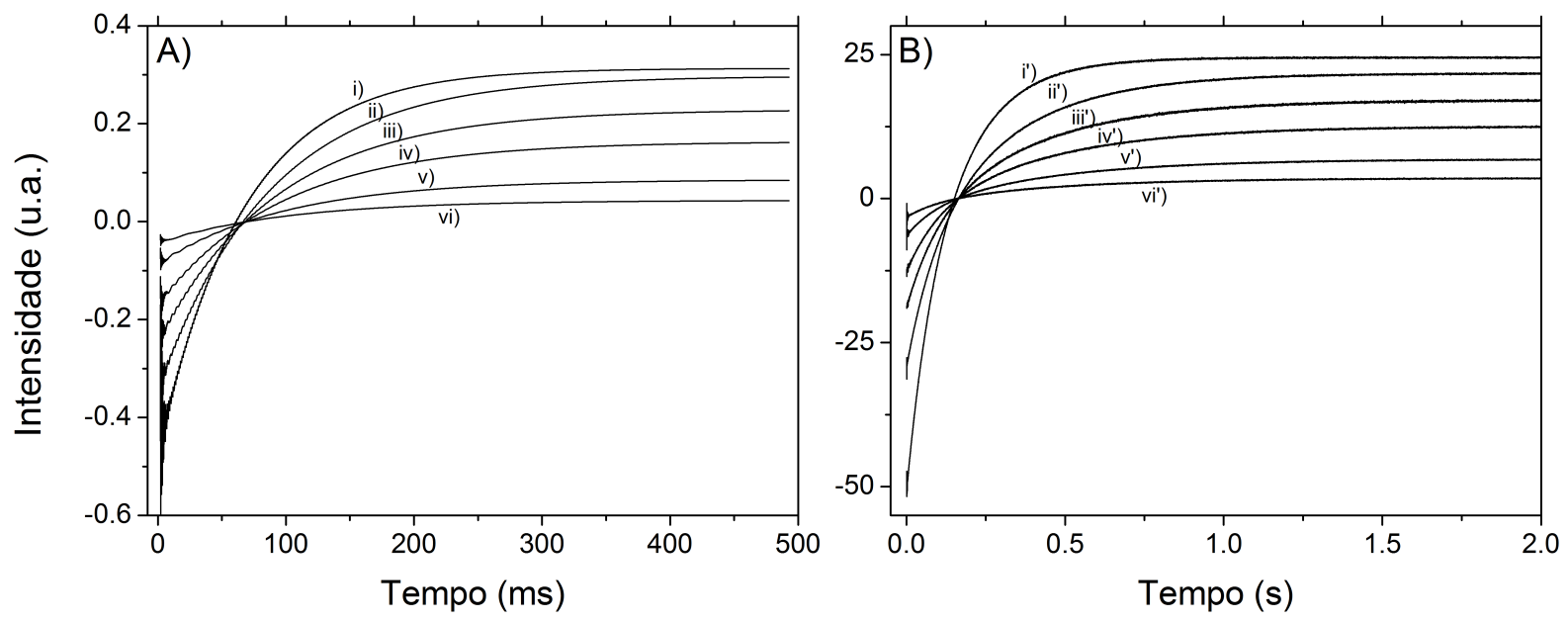

Figura 4.5- Sinais CWFP- $T_{1}$ em A) simulados e em B) experimentais. Os índices i) até vi) são respectivamente para os ângulos $\theta$ de $90^{\circ}, 60^{\circ}, 45^{\circ}, 30^{\circ}, 20^{\circ}, 10^{\circ}$ e $5^{\circ}$.

Fonte: Elaborada pelo autor.

A Fig. 4.6 apresenta uma comparação dos sinais CWFP- $T_{1}$ (cinza) com a IR (pontos pretos), para água deionizada $T_{1}=3,9 \mathrm{~s}$, à $40^{\circ} \mathrm{C}$. $\mathrm{O}$ sinal IR foi adquirido com 36 pontos entre $0,1 \mathrm{~ms}$ e 27 s, espaçados logaritmicamente, com uma aquisição cada, durante tempo total de 9 minutos. O sinal CWFP- $T_{1}$ foi adquirido em aquisição única e contínua (curva cinza), $\theta=10^{\circ}$, durante tempo total de 27 segundos, apresentando razão s/r de 17, que é aproximadamente metade da obtida nos FIDs da aquisição IR (s/r=40), figura interna apresentada.

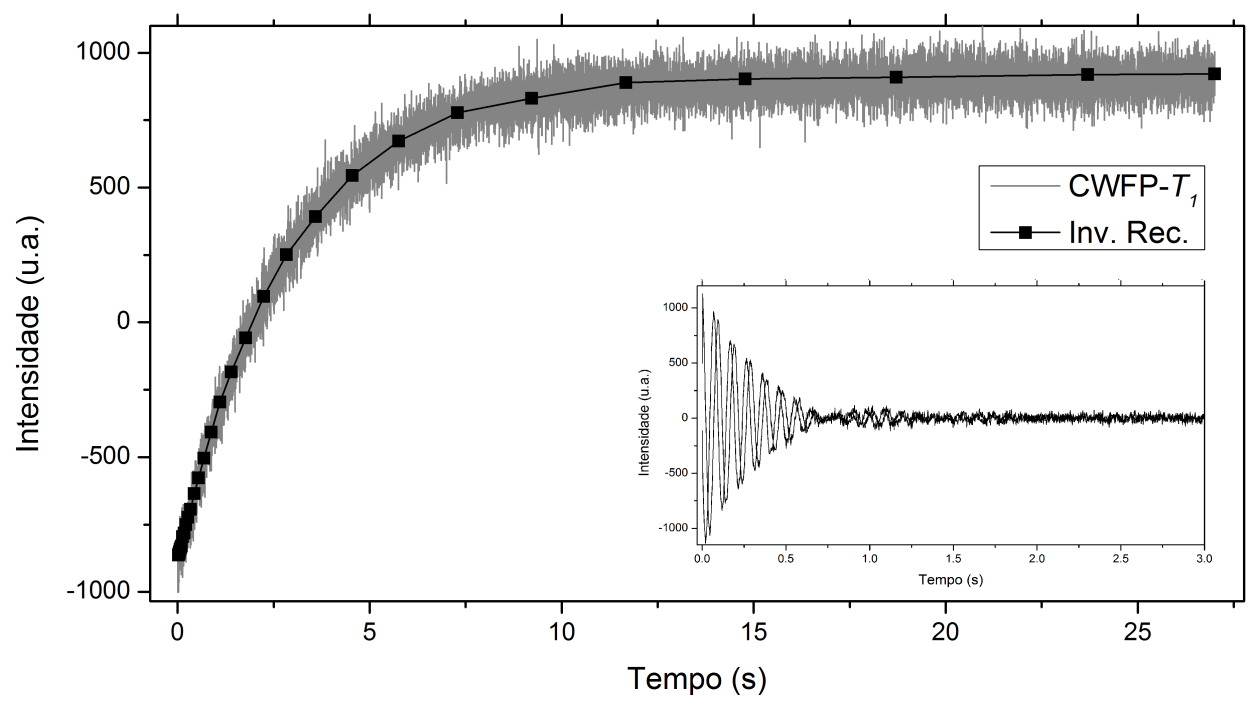

Figura 4.6- Comparação da amplitude dos sinais CWFP- $T_{1}$ (cinza) e IR (pontos pretos), para água deionizada $T_{1}=3,9 \mathrm{~s}$. IR foi adquirido com 36 pontos em 9 minutos de experimento. CWFP- $T_{1}\left(10^{\circ}\right)$ adquirido em aquisição única em 27 segundos, com razão $s / r=17$. A figura interna apresenta um sinal FID da aquisição IR com s/r = 40 .

Fonte: Elaborada pelo autor. 
Para determinação precisa na estimativa de $T_{1}$ de amostras com $T_{1}>>T_{2}$, é necessário utilizar ângulos baixos $\left(<5^{\circ}\right)$, o que consequentemente diminui a razão $s / r$ dos sinais. Apesar disso, o método CWFP- $T_{1}$ mesmo nessas condições resulta na estimativa mais rápida dos tempos de relaxação que a aquisição de um sinal IR com 36 pontos.

Uma segunda característica a ser considerada na CWFP- $T_{1}$ é a ausência da necessidade de se aguardar $5 T_{1}$ entre a realização das médias, pois no final da aquisição CWFP- $T_{1}$ 99,6\% da magnetização já retornou ao eixo z. Assim, a vantagem da CWFP- $T_{1}$ se estabelece no tempo total de experimento e na faixa de tempos de relaxação em estudo. Nos experimentos IR da Tabela 4.1, realizamos 8 médias em uma lista com 36 valores de $\tau$, aguardando $5 T_{1}$ entre cada aquisição. Realizando os experimentos de IR entre 5 minutos e 1 hora de acordo os os tempos de cada amostra. Para a CWFP- $T_{1}$, realizamos 16 médias adquiridas em aquisição única, usando $T_{p}=300 \mu \mathrm{s}$, com tempo total do experimento entre 5 e 20 minutos. A diferença no tempo experimental total entre as sequências IR e CWFP- $T_{1}$ crescem conforme se aumenta o número de pontos na curva IR ou maiores os tempos de relaxação $T_{1}$.

Tabela 4.1- Comparação dos tempos de relaxação (ms) obtidos com as sequências de pulsos IR e CWFP- $T_{1}$ para amostras com diferentes razões $T_{1} / T_{2}$. Os experimentos foram realizados em triplicata com $T_{p}=300 \mu \mathrm{s}$ na CWFP- $T_{1}$ e listas com $36 \tau$ s na aquisição IR.

\begin{tabular}{cccccc}
\hline Espectr. & Amostras & $T_{1}$ IR & CWFP- $_{1}\left(5^{\circ}\right)$ & CWFP- $_{1}\left(10^{\circ}\right)$ & $T_{1} / T_{2}$ \\
\hline \multirow{4}{*}{ Minispec } & Água deionizada & $2818 \pm 36$ & $2827 \pm 18$ & $2812 \pm 9$ & 1,1 \\
& Sol. CuSO & $103,0 \pm 0,3$ & $103 \pm 0,1$ & $102,0 \pm 0,1$ & 1,1 \\
& Sol. MnSO $_{4}$ & $401,0 \pm 0,4$ & $400 \pm 1$ & $389,0 \pm 0,7$ & 4,3 \\
& Sol. Mn(II)-EDTA & $486,0 \pm 1$ & $490 \pm 1$ & $480,0 \pm 0.4$ & 3,5 \\
& Sol. [CuEDTA. $\left.H_{3}\right]^{2-}$ & $605 \pm 4$ & $604 \pm 0.5$ & $573 \pm 0.3$ & 10 \\
\hline \multirow{5}{*}{ SLK1399 } & Água deionizada & $2943 \pm 88$ & $2881 \pm 8$ & $2990 \pm 11$ & 1,3 \\
& Sol. CuSO & $74,0 \pm 0,3$ & $73,0 \pm 0,1$ & $72,0 \pm 0,1$ & 1,2 \\
& Sol. MnSO $_{4}$ & $200 \pm 2$ & $195,0 \pm 0,5$ & $189,0 \pm 0,4$ & 2,7 \\
& Sol. Mn(II)-EDTA & $542 \pm 5$ & $522 \pm 1$ & $502,0 \pm 0.2$ & 1,9 \\
& Sol. [CuEDTA.N H $\left.]_{3}\right]^{2-}$ & $344 \pm 1$ & $340 \pm 1$ & $301 \pm 1$ & 10 \\
\hline
\end{tabular}

Fonte: Elaborada pelo autor.

A Tabela 4.1 apresenta a comparação dos tempos de relaxação $T_{1}$ obtidos pelas sequências de pulsos Inversão Recuperação e CWFP- $T_{1}$ com ângulos de $5^{\circ}$ e $10^{\circ}$, para amostras com diferentes valores de $T_{1}$ entre 0,1 e 3 s e razão $T_{1} / T_{2}$ entre 1 e 10 , adquiridas em dois espectrômetros. Um dos espectrômetros, Minispec mq20, é um modelo de bancada convencional com bore de 10 mm e tempo morto de $12 \mu \mathrm{s}$. O segundo espectrômetro, SLK 1399, tem bore 
de $12 \mathrm{~cm}$ com volume da bobina de $1 \mathrm{~L}$ e tempo morto de $50 \mu \mathrm{s}$.

Os resultados obtidos com as sequências CWFP- $T_{1}$ apresentam-se muito similares aos obtidos por IR. Esses resultados confirmam a robustez do método CWFP- $T_{1}$ na estimativa rápida dos tempos de relaxação longitudinal em amostras mono-exponenciais. A seguir iniciamos a exploração de sistemas mais complexos com distribuição de tempos de relaxação.

\section{CWFP- $T_{1}$ multi-componentes}

A Fig. 4.7 apresenta a Transformada Inversa de Laplace* para os sinais adquiridos com a CPMG, IR e CWFP- $T_{1}$ para uma amostra composta de dois tubos coaxiais (5 e $10 \mathrm{~mm}$ de diâmetro) contendo solução aquosa de $\mathrm{CuSO}_{4}$ (tubo externo) e $\mathrm{MnSO}_{4}$ (tubo interno). Os valores de $T_{2}$ são $93 \mathrm{~ms}$ em ambas soluções e $T_{1}=100 \mathrm{~ms}$ e $418 \mathrm{~ms}$ para o cobre e manganês respectivamente.

A Fig. 4.7 A) apresenta um pico único em 93 ms obtido pela ILT do sinal CPMG do conjunto. A Fig. 4.7 B) apresenta dois picos parcialmente separados obtidos pela ILT do sinal CP-CWFPx-x utilizando pulsos de $90^{\circ}$. O pico em $93 \mathrm{~ms}$ é associado a solução de cobre de razão $T_{1} / T_{2} \approx 1$ e o maior pico em aproximadamente 200 ms associado a solução de manganês com razão $T_{1} / T_{2} \approx 4,2$. Entre Fig. $4.7 \mathrm{C}$ ) e F) é apresentada a separação dos picos na ILT conforme mais reduzido o valor de $\theta$.

Esse deslocamento dos tempos $T^{*}$ com a redução dos ângulos $\theta$ aplicados representa um resultado similar ao da Fig. 4.4, onde cada componente de $T_{2}$ desloca até seu respectivo valor $T_{1}$. Os valores de $T^{*}$ são sempre menores que seus correspondentes valores de $T_{1}$ medidos por IR. Desse modo, os valores de $T^{*}$ calculados pela ILT com a CWFP- $T_{1}$ usando ângulos de $10^{\circ}$ ou $5^{\circ}$ apresentam valores muito próximos de $T_{1}$ das solução de $C u S O_{4}$ e $\mathrm{MnSO}_{4}$ puras.

As Fig. 4.8 e 4.9 apresentam a comparação da distribuição dos tempos de relaxação para as amostras de carne com gordura, gordura, pasta de dendê e macadâmia. Os sinais foram obtidos com as sequências CPMG, IR com 36 e 144 pontos e CWFP- $T_{1}$ com $5^{\circ}$ e $T_{p}=300$ $\mu \mathrm{s}$.

Na Fig. $4.8 \mathrm{~A}$ ) vemos que ao processar o sinal IR e CWFP- $T_{1}$ com mesmo valor no

\footnotetext{
* Calculada com o código apresentado no apêndice 8.
} 


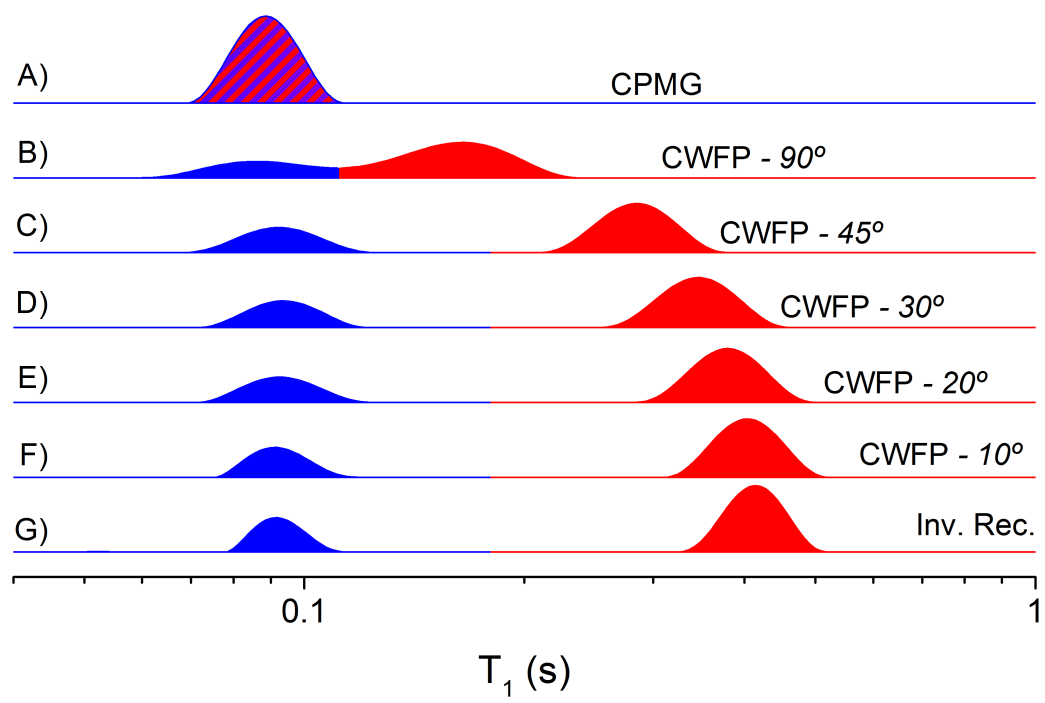

Figura 4.7- Espectros ILT obtidos pelos sinais CPMG, CWFP- $T_{1}$ e IR de duas soluções $\mathrm{CuSO}_{4}$ e $\mathrm{MnSO}_{4}$ adquiridas simultaneamente em tubos concêntricos de 5 e $10 \mathrm{~mm}$ diâmetro. ILT dos sinais A) CPMG e G) Inversão Recuperação. Entre B) e F) ILT de sinais CP-CWFPx-x com $\theta=90^{\circ}, 45^{\circ}$, $30^{\circ}, 20^{\circ}$ e $10^{\circ}$.

Fonte: Elaborada pelo autor.

parâmetro de regularização da ILT $(\alpha=10)$, a sequência CWFP- $T_{1}$ resulta em maior resolução, onde o pico em $0,1 \mathrm{~s}$ da IR $(\alpha=10)$ se divide em dois. Essa hipótese é confirmada quando reprocessamos o sinal IR $\operatorname{com} \alpha=0,1$ e obtemos três picos, similar a distribuição da CWFP- $T_{1}$. Em B) é apresentada a comparação da distribuição dos tempos de relaxação para gordura.
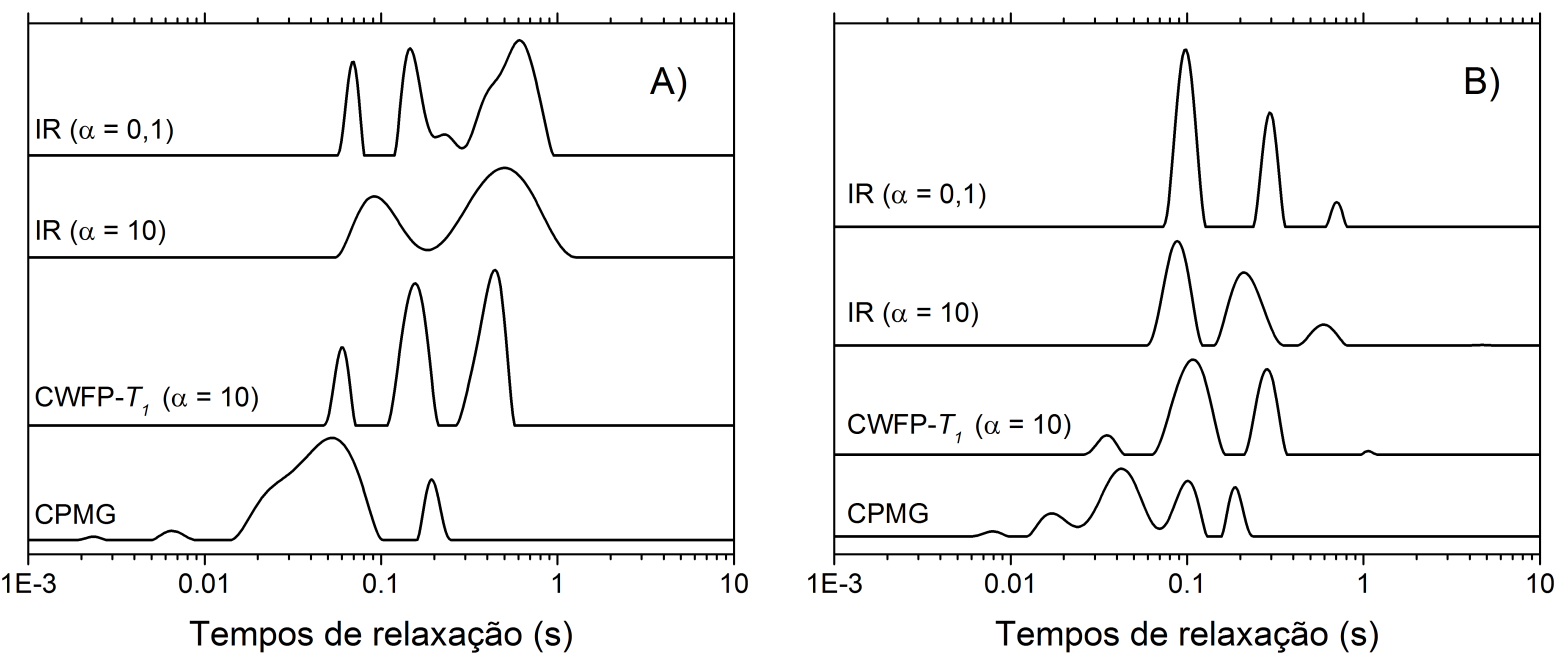

Figura 4.8- Comparação da distribuição dos tempos de relaxação para amostras de A) carne com gordura e em B) apenas gordura. Os sinais IR foram obtidos com 36 pontos espaçados logaritmamente.

Fonte: Elaborada pelo autor.

A Fig. 4.9 apresenta a comparação dos tempos de relaxação para amostras de A) pasta de Dendê e B) Macadâmia. Nesses casos foram realizadas as aquisições da curva IR com 36 pontos e 144 pontos e todas as curvas processadas $\operatorname{com} \alpha=10$. Nota-se que no Dendê A) a 
CWFP- $T_{1}$ apresenta quatro picos bem definidos na região entre 50 e 500 ms. Nos sinais ILT das curvas IR com 36 e 144 pontos, ambos os casos resultaram em uma distribuição larga de picos na mesma região, porém não definidos. A mesma análise pode ser feita no resultado da macadâmia em B) nas regiões entre 40 e 400 ms.

Esses resultados reforçam a hipótese de maior resolução obtida na sequência CWFP- $T_{1}$ frente as aquisições IR. Associamos essa observação ao fato de que os sinais CWFP- $T_{1}$ apresentam centenas de pontos a mais em seu sinal. Nos casos apresentados acima, os sinais IR possuem 36 ou 144 pontos, frente a entre 5 mil e 15 mil pontos nas curvas CWFP- $T_{1}$.
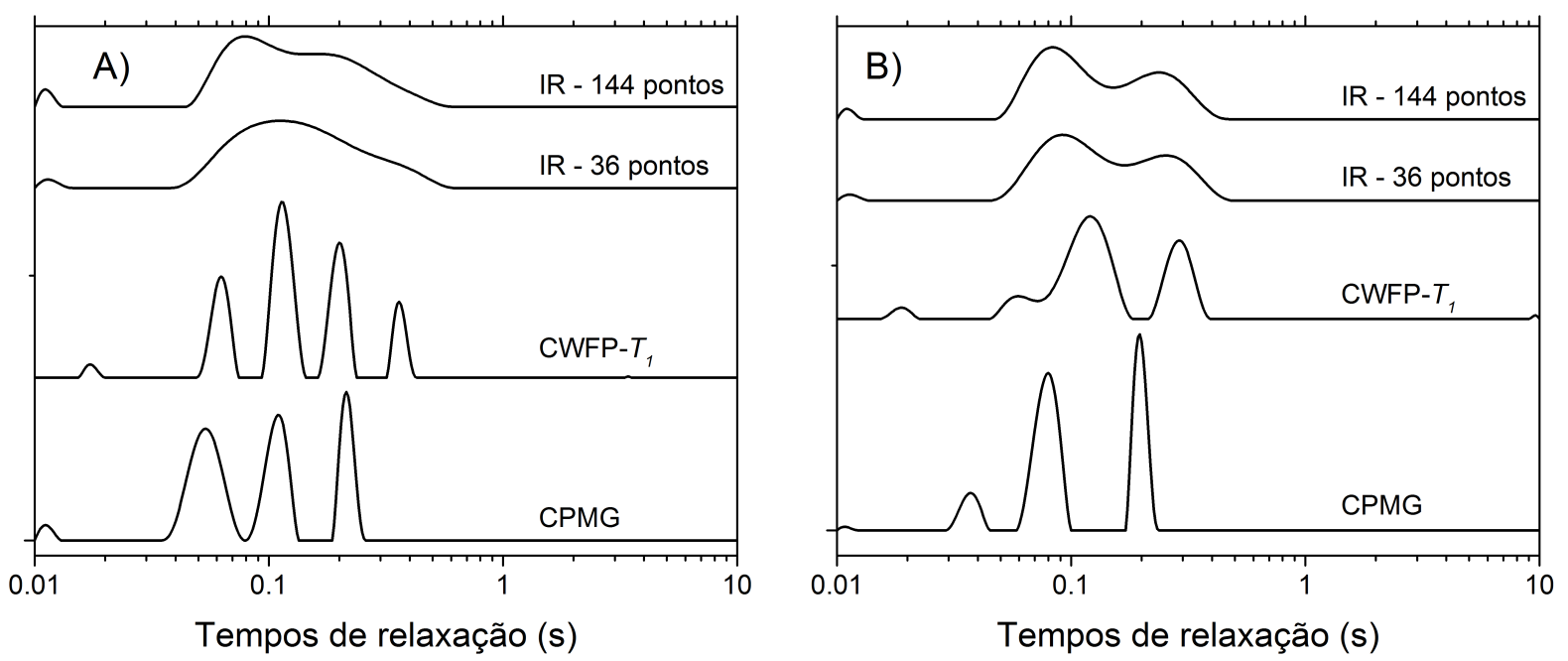

Figura 4.9- Comparação da distribuição dos tempos de relaxação para amostras de A) Dendê e em B) Macadâmia. Os sinais IR foram obtidos com 36 e 144 pontos espaçados logaritmamente. Os sinais CWFP- $T_{1}$ foram adquiridos com $T_{p}=300 \mu \mathrm{s} \mathrm{com} 15$ mil pontos na curva. Os processamento ILT foram realizados com parâmetro de regularização $\alpha=10$ e 400 pontos.

Fonte: Elaborada pelo autor.

Muitos estudos em RMN de baixa resolução utilizam a sequência CPMG devido sua robustez e menor tempo experimental total frente a IR, porém como fica evidenciado nos resultados das carnes com gordura Fig. 4.8 A) e apenas gordura em B), análises pela relaxação $T_{1}$ pode apresenta significativas informações no estudo do sistemas com forte dependência com $T_{1} \cdot{ }^{98}$

Com esses resultados concluímos que a sequência CWFP- $T_{1}$ é uma via simples, rápida e confiável para determinação da distribuição de tempos de relaxação $T_{1}$. Essa sequência pode ser muito útil na análise de processos e produtos em escala industrial, principalmente em amostras com forte dependência com $T_{1}$, onde métodos rápidos e quantitativos são altamente desejáveis. 


\subsection{Estado Estacionário em magnetos compactos}

O projeto de pesquisa no exterior consistiu em investigar e desenvolver métodos rápidos de relaxometria baseados em sequências de pulsos no estado estacionário semelhantes a CWFP. As sequências de pulsos Driven-Equilibrium CPMG (DECPMG) e Split $180^{\circ}$ foram estudas e implementadas nos sistemas magnéticos compactos: mini-Halbach e MOUSE-NMR. As sequências de pulsos Driven-Equilibrium CPMG (DECPMG) e Split $180^{\circ}$ foram estudas e implementadas nos sistemas magnéticos compactos: mini-Halbach e MOUSE-NMR.

\section{Magnetos compactos}

As técnicas espectroscópicas de RMN são baseadas na utilização de equipamentos de custo elevado, principalmente devido a utilização de líquidos criogênicos em magnetos supercondutores, necessários para obtenção de campos magnéticos altos e de alta homogeneidade.

O desenvolvimento de magnetos homogêneos baseados as ímãs permanentes vem evoluindo rapidamente nos últimos anos, devido a melhor qualidade das peças de ímãs produzidos e novas técnicas de construção e shimming de campo. ${ }^{99,100}$ Esses sistemas compactos tem custo final substancialmente menor, tornando tais sensores uma ferramenta atraente para o setor industrial, com enorme potencial para controle de qualidade, processos, produtos e monitoramento em tempo real de reações químicas. ${ }^{58,101}$

Nesse contexto, o desenvolvimento de métodos rápidos para relaxometria e espectroscopia nesses novos sistemas compactos é de grande relevância e pode ser alcançado com as técnicas de estado estacionário. No projeto foram utilizados dois magnetos, sendo um magneto unilateral (Single Sided NMR-MOUSE) 62,102,103 que consiste de um magneto com campo magnético externo com alto grau de inomogeneidade de campo, e o segundo um magneto no formato Halbach $^{58}$, denominado aqui mini-Halbach por apresentar dimensões de apenas $15 \mathrm{~cm}$ por 8 $\mathrm{cm}$, desenvolvido para tubos de $5 \mathrm{~mm}$ e com campo magnético homogêneo. ${ }^{99}$

A Fig. 4.10 apresenta as instalações do laboratório com o espectrômetro KEA junto com o Singled-Sided NMR-MOUSE com campo magnético de 0,2 T, correspondendo a uma frequência para hidrogênio de $9 \mathrm{MHz}$, com gradiente de campo de $20 \mathrm{~T} / \mathrm{m}$. A Fig. 4.11 apresenta a montagem com o magneto mini-Halbach com campo $0,7 \mathrm{~T}$, frequência do hidrogênio de 
$26 \mathrm{MHz}$, com boa homogeneidade de campo e resolução sub-ppm. Bobinas de shimming são utilizadas para melhorar a homogeneidade de campo e as amostra são colocadas em tubos de $5 \mathrm{~mm}$.

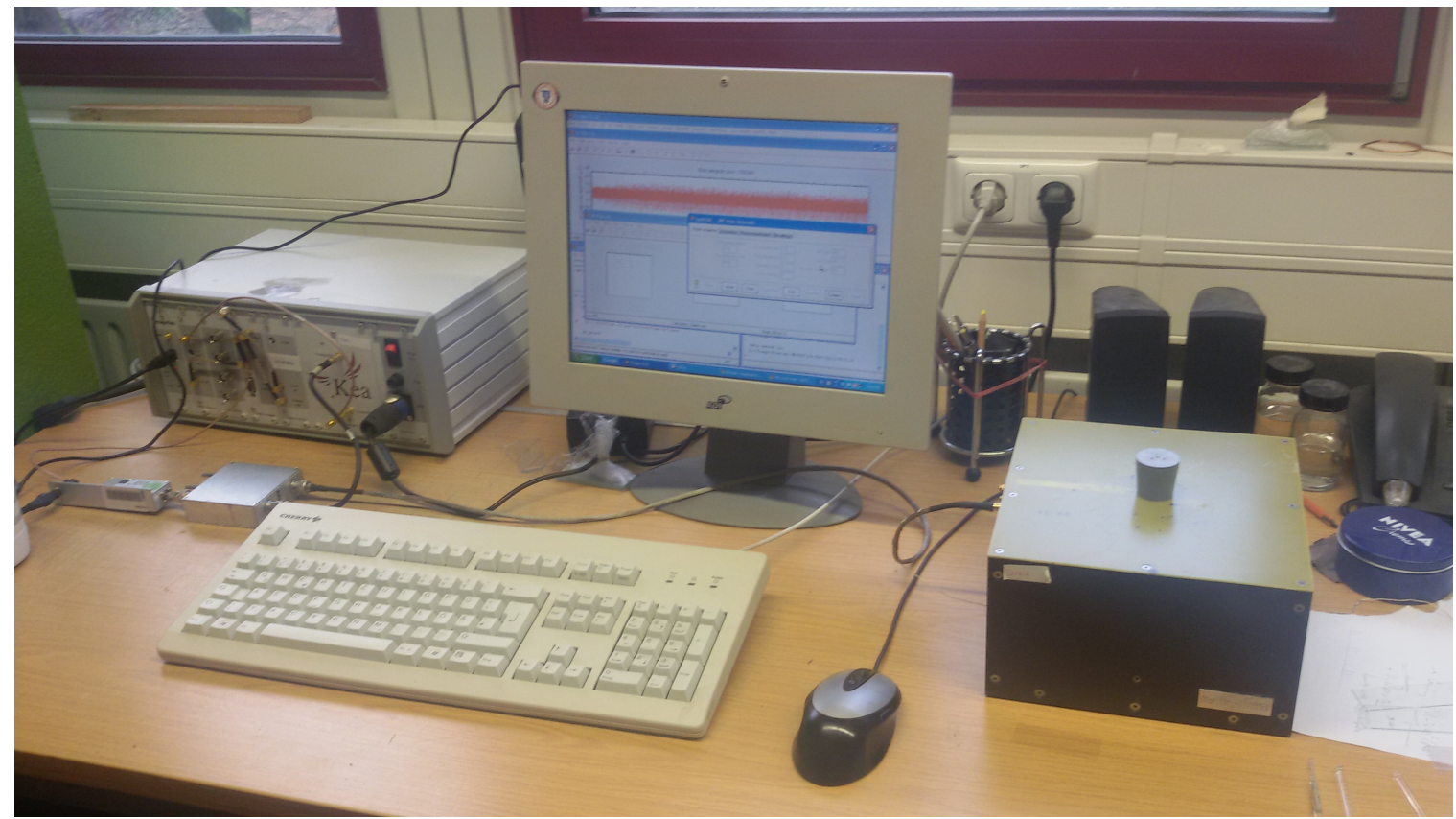

Figura 4.10- Montagem do espectrômetro KEA com o magneto unilateral Singled-Sided NMR-MOUSE. Fonte: Elaborada pelo autor.

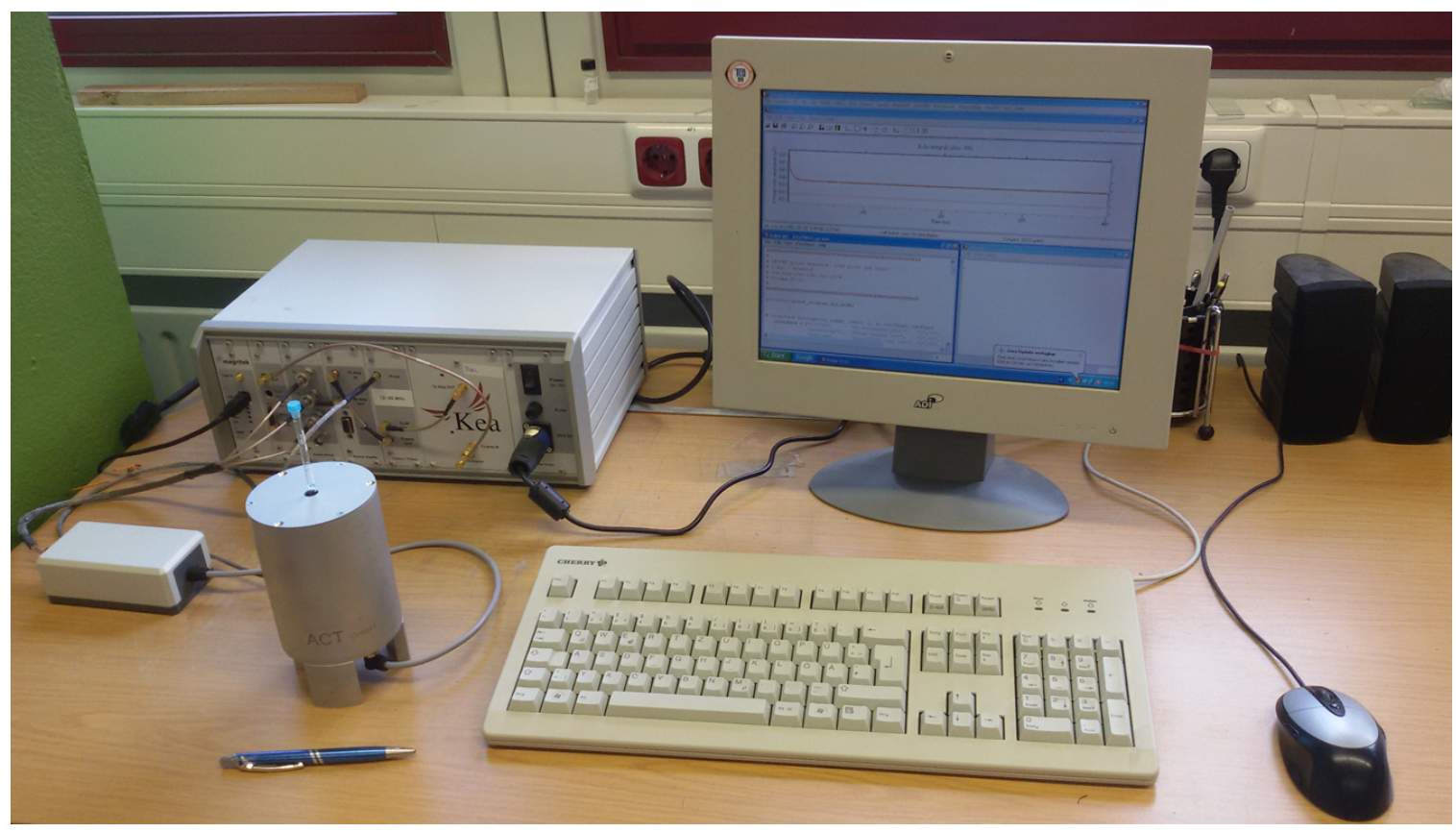

Figura 4.11- Montagem do espectrômetro KEA com o magneto mini-Halbach. Fonte: Elaborada pelo autor. 
DECPMG para aplicações práticas nos sistemas magnéticos acima descritos. Ambas as sequências foram recentemente propostas pela equipe de $M$. Hurlimman et al. da empresa Schlumberger-Doll Research no desenvolvimento de novos métodos de RMN para o setor petrolífero. ${ }^{35,36}$ As sequências são baseadas em técnicas no estado estacionário da magnetização, com características similares a CWFP, sendo que a Split $180^{\circ}$ foi desenvolvida para campos magnéticos com alto grau de inomogeneidade e a DECPMG para campos homogêneos.

As sequências de pulsos foram implementadas no software Prospa v 3.2 no espectrômetro KEA, que controla ambos os magnetos, como apresentado nas Fig. 4.10 e Fig. 4.11. Os resultados obtidos são apresentados nas próximas seções.

\subsubsection{Split $180^{\circ}$ e Butterfly Pathways}

A implementação da sequência de pulsos Split $180^{\circ}$ foi realizada seguindo de perto as descrições das referências e patentes ${ }^{36,78,80,81}$ dos cientistas associados a companhia Schlumberger-Doll Research. O método foi proposto para relaxometria e atuação em campos magnéticos com alto grau de inomogeneidade.

Na Fig. 2.13 vimos que a sequência Split $180^{\circ}$ é muito semelhante a uma CPMG, sendo que os pulsos de $180^{\circ}$ foram divididos em dois pulsos de $90^{\circ}$ e espaçados por um tempo $\tau_{2}$. A aquisição dos ecos ocorrem apenas nos intervalos $\tau_{1}$ e diferentes esquemas de fases são utilizados nos pulsos $\phi_{0}, \phi_{1}$ e $\phi_{2}$. Para $\phi_{1}=\phi_{2}$ a Split $180^{\circ}$ é denominada Simétrica e quando $\phi_{1}=-\phi_{2}$ temos a Split $180^{\circ}$ Anti-simétrica. A Tabela 4.2 apresenta o esquema de fase dos pulsos e receptor da Split simétrica e anti-simétrica, na aquisição dos sinais CPMG-like e Steady-State.

Tabela 4.2- Esquema de ciclo de fases para Split $180^{\circ}$ simétrica e anti-simétrica para aquisição do sinal steady-state ou cpmg-like. De cima pra baixo fase dos pulsos $\phi_{0}, \phi_{1}, \phi_{2}$ e receptor.

\begin{tabular}{cccc}
\hline \multicolumn{2}{c}{ Simétrica } & \multicolumn{2}{c}{ Anti-simétrica } \\
steady-state & cpmg-like & steady-state & cpmg-like \\
\hline $0, \pi$ & 0,0 & $\frac{\pi}{2}, \frac{-\pi}{2}$ & $\frac{\pi}{2}, \frac{3 \pi}{2}$ \\
$0, \pi$ & $\pi, 0$ & 0,0 & 0,0 \\
0,0 & $0, \pi$ & 0,0 & 0,0 \\
0,0 & 0,0 & 0,0 & $0, \pi$ \\
\hline
\end{tabular}

Fonte: Elaborada pelo autor.

As sequências Split criam dois sinais simultâneos. Um decai similar a uma CPMG que 
fornece informação de $T_{2, e f}$ (efetivo) chamado sinal CPMG-like, e outro que cresce até um estado estacionário onde a amplitude é função da razão dos tempos de relaxação $T_{1} / T_{2}$, descrito na equação 2.5.10.

A Fig. 4.12 apresenta sinais Split $180^{\circ}$ adquiridos de uma amostra água dopada com cobre $T_{1}=T_{2}=18 \mathrm{~ms}$ adquiridos com $\tau_{1}=400 \mu \mathrm{s}, \tau_{2}=100 \mu \mathrm{s}, \phi_{0}=y \phi_{1}=\phi_{2}=x, 512$ ecos e 512 médias no magneto NMR-MOUSE de $9 \mathrm{MHz}$. Em A) apresentamos a amplitude das componentes real e imaginária adquiridas em função do tempo. A componente real (linha preta) apresenta decaimento CPMG-like da magnetização com constante de tempo $T_{2, e f}$ e a parte imaginária (linha vermelha) a amplitude dos ecos que crescem até o estado estacionário com amplitude que depende da razão $T_{1} / T_{2}$.

Na Fig. 4.12 B) e C) apresentamos o formato completo dos ecos obtidos na janela de aquisição com $300 \mu$ s dentro do intervalo $\tau_{1}$. B) mostra que os ecos do decaimento CPMGlike apresentam estrutura com três ecos principais, gerados devido as múltiplas refocalizações da magnetização. A posição desses ecos na janela de aquisição é controlada com os tempos $\tau_{1}$ e $\tau_{2}$ além de mudanças causada pelas fases $\phi_{0}, \phi_{1}$ e $\phi_{2}$ que serão discutidas a frente. Em C) apresentamos o formato dos ecos no estado estacionário, que apresentam duas componentes predominantes e simétricas ao centro da janela de aquisição, com amplitudes máximas na componente imaginária.
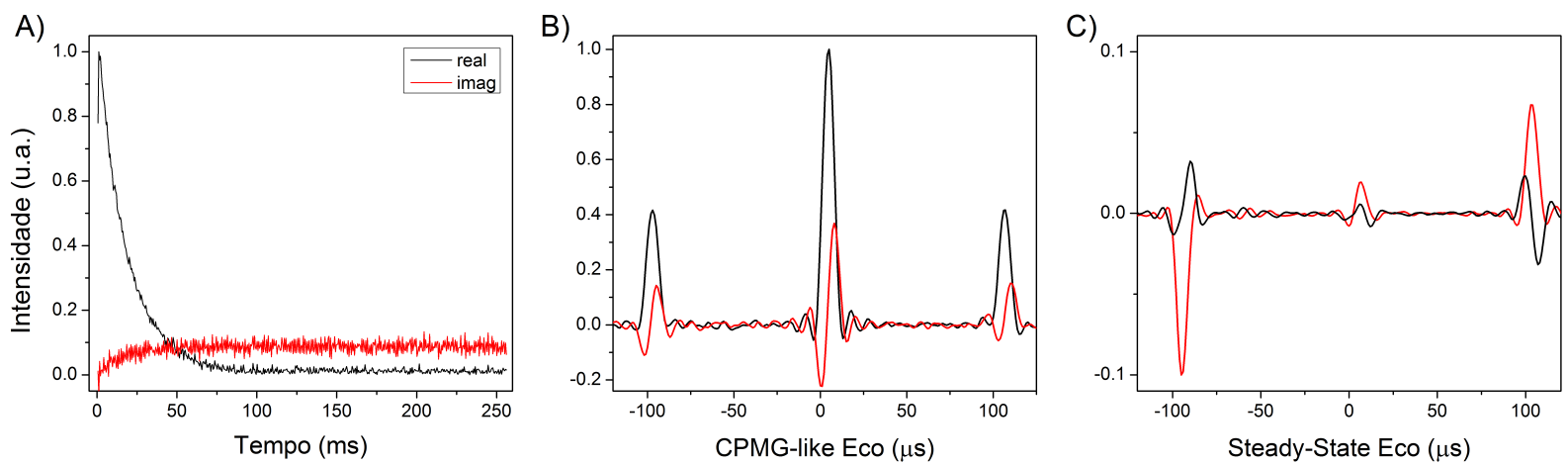

Figura 4.12- Sinais adquiridos com a Split $180^{\circ}$ para amostra de água dopada com cobre $T_{1} / T_{2}=1$. Em A) decaimento e crescimento dos ecos na Split $180^{\circ}$ no tempo. Em B) apresentamos o formato dos ecos na componente real da magnetização e em C) os ecos que crescem ao estado estacionário, usando $\tau_{1}=400 \mu \mathrm{s}, \tau_{2}=100 \mu \mathrm{s}, 512$ ecos e 512 médias no magneto NMR-MOUSE de $9 \mathrm{MHz}$.

Fonte: Elaborada pelo autor.

Para estudarmos as diversas possibilidades da sequência Split $180^{\circ}$ realizamos uma série de simulações numéricas com a descrição teórica da seção 2.5.2. A Fig. 4.13 apresenta simulações 
da sequência Split $180^{\circ}$ submetida a diferente valores de campo magnético inomogêneo $\overrightarrow{B_{0}} \hat{z}$, definidos com picos de largura de meia altura de $F W H I=20 \mathrm{KHz}, 10 \mathrm{KHz}, 2 \mathrm{KHz}$ e 10 Hz. Resultados mostram que conforme maior a inomogeneidade de campo magnético mais estreitos são os ecos formados (preto) e maior a amplitude dos ecos formados na componente CPMG-like, Fig. 4.13 A), e no sinal no estado estacionário Fig. 4.13 B).
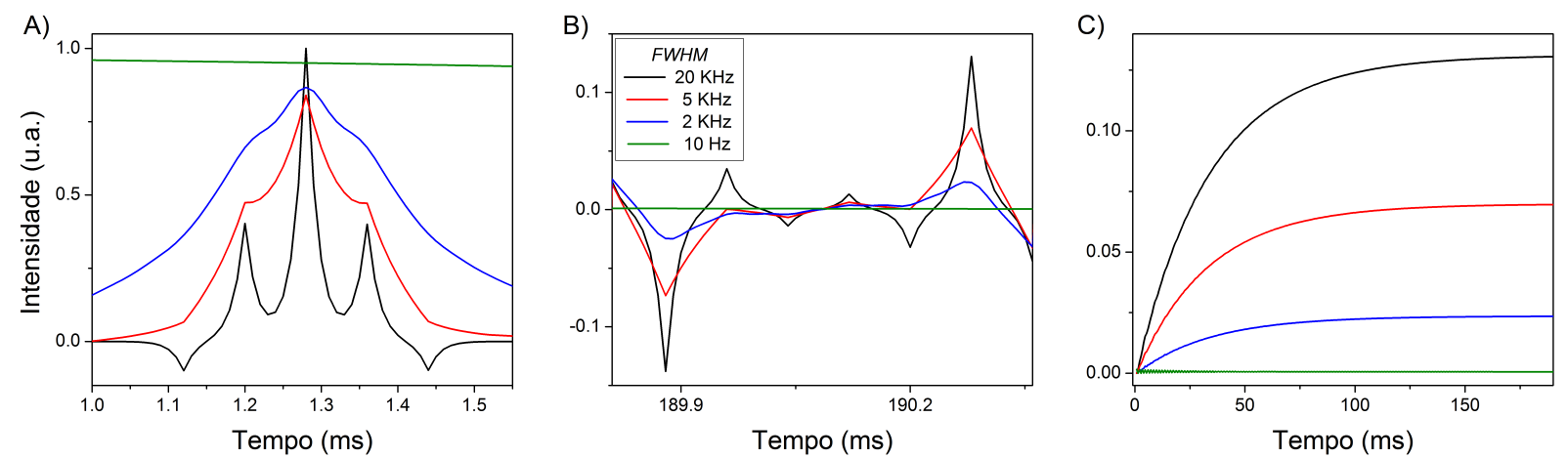

Figura 4.13- Simulação numérica da Split $180^{\circ}$ simétrica em função da inomogeneidade de campo $\vec{B}_{0} \hat{z}$ com largura de meia altura $F W H I=20 \mathrm{KHz}, 10 \mathrm{KHz}, 2 \mathrm{KHz}$ e $10 \mathrm{~Hz}$, usando $\tau_{1}=300 \mu \mathrm{s}, \tau_{2}=100$ $\mu \mathrm{s}, \phi_{0}=y, \phi_{1}=\phi_{2}=x$. Em A) e B) apresentamos o formato dos ecos do sinal CPMG-like e no estado estacionário respectivamente. C) apresenta o crescimento das amplitude dos ecos indo ao estado estacionário.

Fonte: Elaborada pelo autor.

A Fig. 4.13 C) compara a amplitude os ecos indo ao estado estacionário em função da inomogeneidade de campo $\vec{B}_{0} \hat{z}$. Note que em campos magnéticos mais homogêneos a sequência Split $180^{\circ}$ resulta em ecos mais largos, de modo que para adquiri-los seria necessário aumentar os tempos entre pulsos $\tau_{1}$ e $\tau_{2}$, até limites onde $\tau_{1,2}$ não seriam muito menores que os tempos de relaxação e portanto não formaria o estado estacionário. Desse modo a Split $180^{\circ} \mathrm{em}$ campos magnéticos homogêneos se comportam como uma CPMG apresentando apenas o sinal que decai a zero e amplitude nula no estado estacionário (verde).

As fases $\phi_{0}, \phi_{1}$ e $\phi_{2}$ dos pulsos da Split $180^{\circ}$ controlam se os ecos gerados são positivos ou negativos. A Fig. 4.13 foi simulada usando $\phi_{0}=y, \phi_{1}=\phi_{2}=x$, e denominamos essa sequência de Split $180^{\circ}$ Simétrica. Para a situação onde $\phi_{0}=x, \phi_{1}=-x$ e $\phi_{2}=x$ os ecos da Split $180^{\circ}$ se formam de acordo com a Fig. 4.14, e a sequência é denominada Split $180^{\circ}$ Anti-simétrica.

A Fig. 4.14 apresenta simulações numéricas da Split $180^{\circ}$ anti-simétrica em função da inomogeneidade de campo $\vec{B}_{0} \hat{z}$ com largura de meia altura $F W H I=20 \mathrm{KHz}, 10 \mathrm{KHz}, 2 \mathrm{KHz}$ 
e $10 \mathrm{~Hz}$, usando $\tau_{1}=300 \mu \mathrm{s}, \tau_{2}=100 \mu \mathrm{s}$. Na Fig. $4.14 \mathrm{~A}$ ) e B) apresentamos o formato dos ecos do sinal CPMG-like e no estado estacionário, que quando comparados com os ecos Split $180^{\circ}$ simétrica (Fig. 4.13) vemos que os ecos gerados apresentam a mesma posição na escala da janela de aquisição, porém com amplitudes de sinal diferentes.
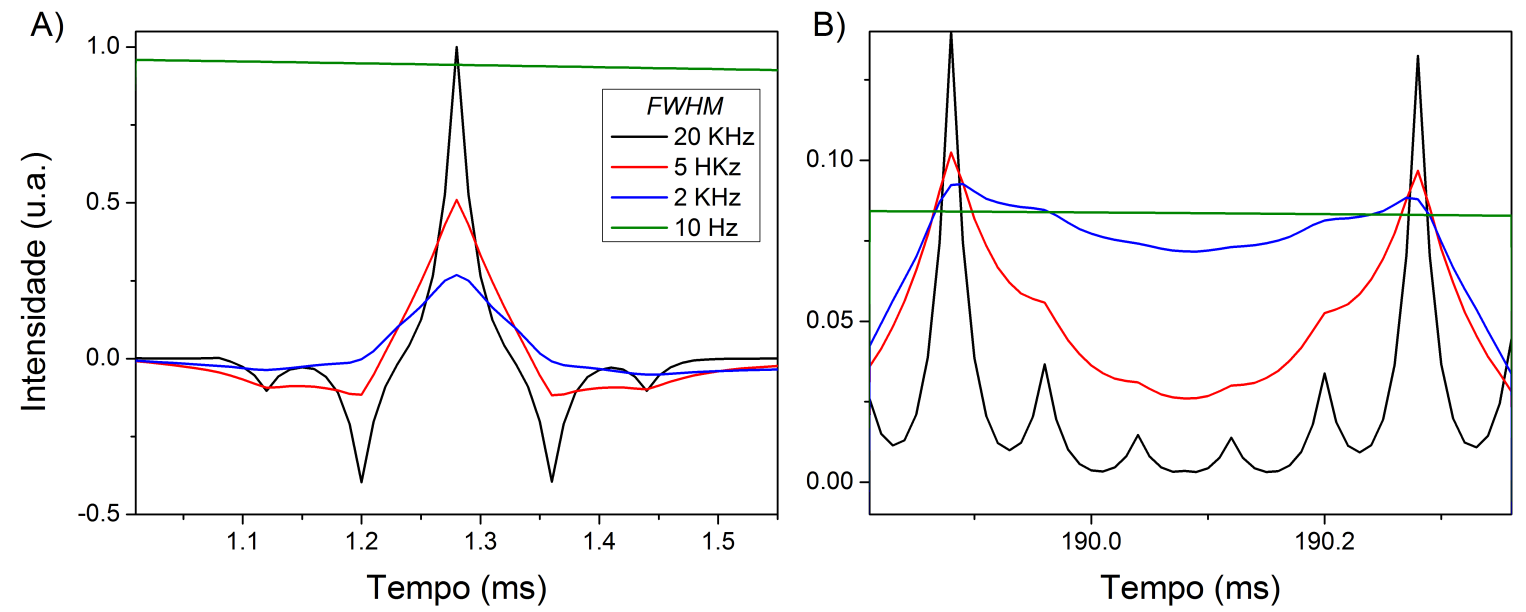

Figura 4.14- Simulação numérica da Split $180^{\circ}$ anti-simétrica em função da inomogeneidade de campo $\overrightarrow{B_{0}} \hat{z}$ com largura de meia altura $F W H I=20 \mathrm{KHz}, 10 \mathrm{KHz}, 2 \mathrm{KHz}$ e $10 \mathrm{~Hz}$, usando $\tau_{1}=300 \mu \mathrm{s}, \tau_{2}$ $=100 \mu \mathrm{s}, \phi_{0}=y, \phi_{1}=-x$ e $\phi_{2}=x$. Em A) e B) apresentamos o formato dos ecos do sinal CPMG-like e no estado estacionário respectivamente.

Fonte: Elaborada pelo autor.

Uma propriedade peculiar das sequências Split $180^{\circ}$ é que a amplitude do estado estacionário é independente dos valores específicos de $T_{1}$ ou $T_{2}$, sendo apenas função da razão $T_{1} / T_{2}$. Na Fig. 4.15 apresentamos sinais experimentais adquiridos com a Split simétrica para três amostras de água dopada com diferentes concentrações de cobre, resultando em tempos de relaxação $T_{2}$ igual a A) $3 \mathrm{~ms}$, B) $30 \mathrm{~ms}$ e C) $160 \mathrm{~ms}$. O decaimento da componente real nos três gráficos apresentam o decaimento do $T_{2, \text { ef }}$ de cada, entretanto a amplitude do estado estacionário de todos são iguais, uma vez que todos tem razão $T_{1} / T_{2}=1$.

Dessa forma a Fig. 4.15 evidência que a Split $180^{\circ}$ é uma poderosa ferramenta para se obter a informação de $T_{2}$ e $T_{1} / T_{2}$ em um experimento rápido e simples em campos magnéticos inomogêneos.

\section{Split $180^{\circ}$ anti-simétrica}

A amplitude do estado estacionário pode ser otimizada através de ajustes dos parâmetros $\tau_{1}$ e $\tau_{2}$. A Fig. 4.16 apresenta sinais adquiridos com a sequência simétrica e anti-simétrica fixando 

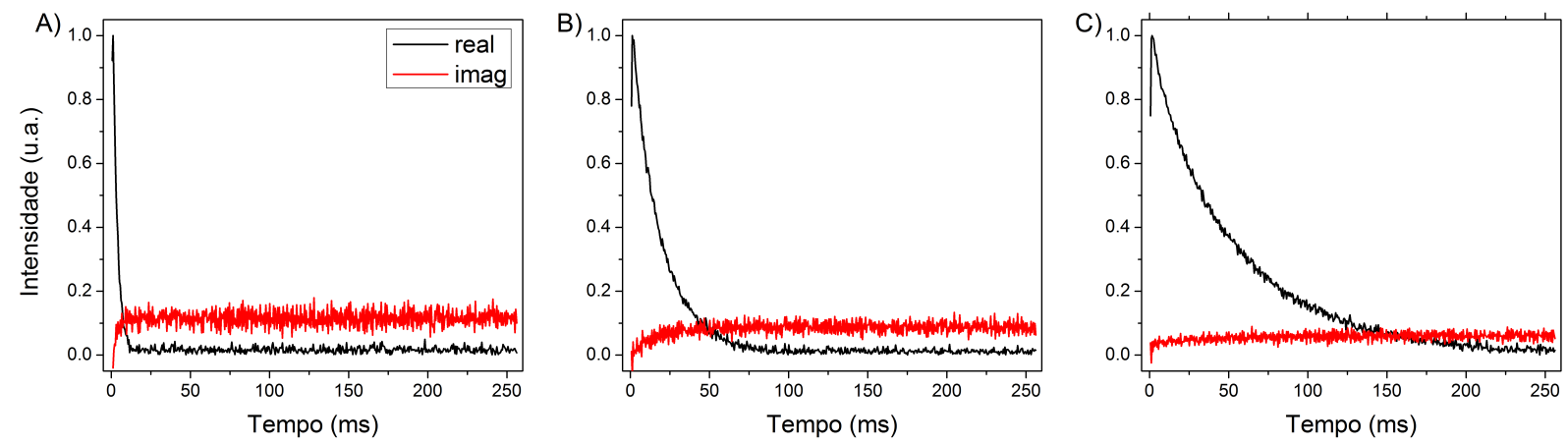

Figura 4.15- Split $180^{\circ}$ simétrica para amostras de água dopada com cobre de diferentes valores de $T_{2}$ A) 3 $\mathrm{ms}$, B) $30 \mathrm{~ms}$ e C) $160 \mathrm{~ms}$. Note que a amplitude no estado estacionário é independente do valor de $T_{2}$ sendo a mesma amplitude uma vez que todos tem razão $T_{1} / T_{2}=1$.

Fonte: Elaborada pelo autor.

$\tau_{1}=400 \mu \mathrm{s}$ para os valores de $\tau_{2}=100,147$ e $200 \mu \mathrm{s}$. A primeira linha apresenta os sinais Split $180^{\circ}$ simétrica $\left(\phi_{1}=\phi_{2}=x\right)$ onde vemos que quando $\tau_{1}=2 \tau_{2}$, em C), obtemos um mínimo de intensidade na amplitude do estado estacionário. Isso ocorre devido a sobreposição destrutiva dos ecos apresentados na simulação da Fig. 4.13. O efeito inverso é observado na segunda linha, onde apresentamos os sinais Split $180^{\circ}$ anti-simétrica $\left(\phi_{1}=x, \phi_{2}=-x\right)$ onde agora os ecos da simulação Fig. 4.13 estão todos com mesma fase e se sobrepõem construtivamente, obtendo um máximo de sinal em $\tau_{1}=2 \tau_{2}, \mathrm{~F}$ ).
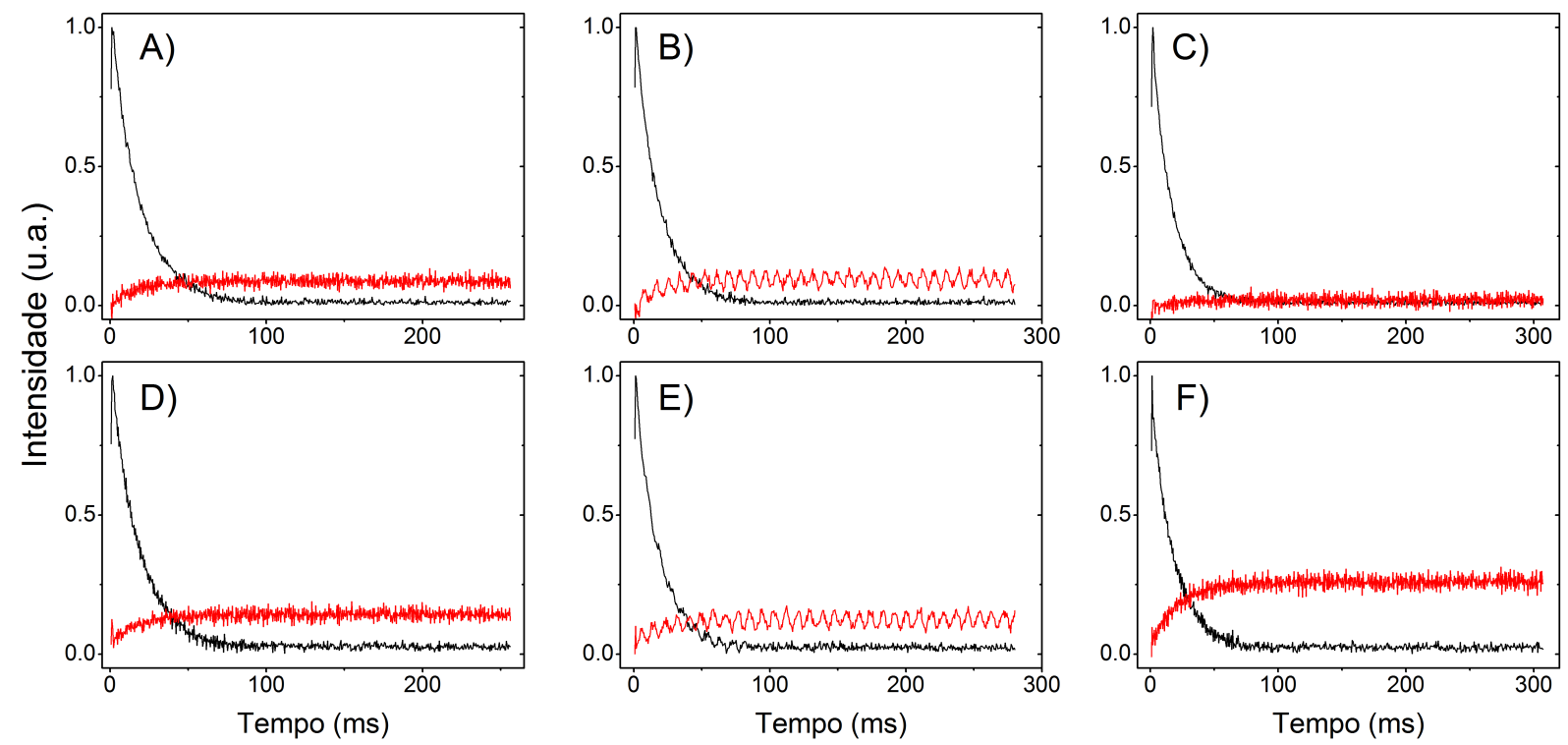

Figura 4.16- A linha superior apresenta sinais Split $180^{\circ}$ simétrica com $\tau_{1}=400 \mu \mathrm{s}$ e A) $\left.\tau_{2}=100, \mathrm{~B}\right) \tau_{2}$ $=147 \mathrm{e} \mathrm{C}) \tau_{2}=200 \mu \mathrm{s}$. A linha inferior apresenta sinais de Split $180^{\circ}$ anti-simétrica com $\tau_{1}$ $=400 \mu \mathrm{s} \mathrm{e} \mathrm{D)} \tau_{2}=100$, E) $\tau_{2}=147$ e F) $\tau_{2}=200 \mu \mathrm{s}$. Experimentos foram realizados com amostra de água dopada com cobre com $T_{1}=T_{2}=30 \mathrm{~ms}$, 512 ecos e 512 médias.

Fonte: Elaborada pelo autor.

Realizamos uma séria de simulações e experimentos e verificamos que a condição da Split 
$180^{\circ}$ anti-simétrica com $\tau_{1}=2 \tau_{2}$ se obtém a melhor amplitude dos sinais no estado estacionário, sendo a Split $180^{\circ}$ com melhor razão s/r e portanto nossa escolhida nas aplicações práticas. Mais detalhes sobre a formação e sobreposição construtiva e destrutivas dos ecos nas Split $180^{\circ}$ Simétrica e Anti-simétrica será apresentada na seção seguinte.

Exploramos agora a dependência do sinal Split com a razão dos tempos de relaxação $T_{1} / T_{2}$ em diferentes materiais. A Fig. 4.17 apresenta os sinais Split $180^{\circ}$ anti-simétrica com $\tau_{1}=$ $400 \mu$ s e $\tau_{2}=200 \mu$ s para amostras de água dopada com cobre e três tipos de borracha. Na Fig. 4.17 A) temos a razão $T_{1} / T_{2}=1$ para água dopada. B) sinais adquiridos da Borracha B. (branca) com $T_{1} / T_{2}=2,7$, C) Borracha C. (cinza) com $T_{1} / T_{2}=1,9$ e D) Borracha P. (preta) com $T_{1} / T_{2}=6,7$. Os tempos de relaxação dos materiais são apresentados na Tabela 4.3.

Tabela 4.3- Tempos de relaxação das amostras com as sequências de pulsos CPMG e Inversão Recuperação.

\begin{tabular}{ccccccc}
\hline Tempos de Rel. & Água dop. & Borracha B. & Borracha C. & Borracha P. & Queijo 1 & Queijo 2 \\
\hline$T_{1}(\mathrm{~ms})$ & 30,0 & 44,0 & 16,0 & 20,0 & 95,0 & 100,0 \\
$T_{2}(\mathrm{~ms})$ & 30,0 & 23,0 & 6,0 & 3,0 & $10,0 \mid 54,0$ & $16,0 \mid 51,0$ \\
$T_{1} / T_{2}$ & 1,0 & 1,9 & 2,7 & 6,7 & $9,5 \mid 1,8$ & $6,3 \mid 2,0$ \\
\hline
\end{tabular}

Fonte: Elaborada pelo autor.
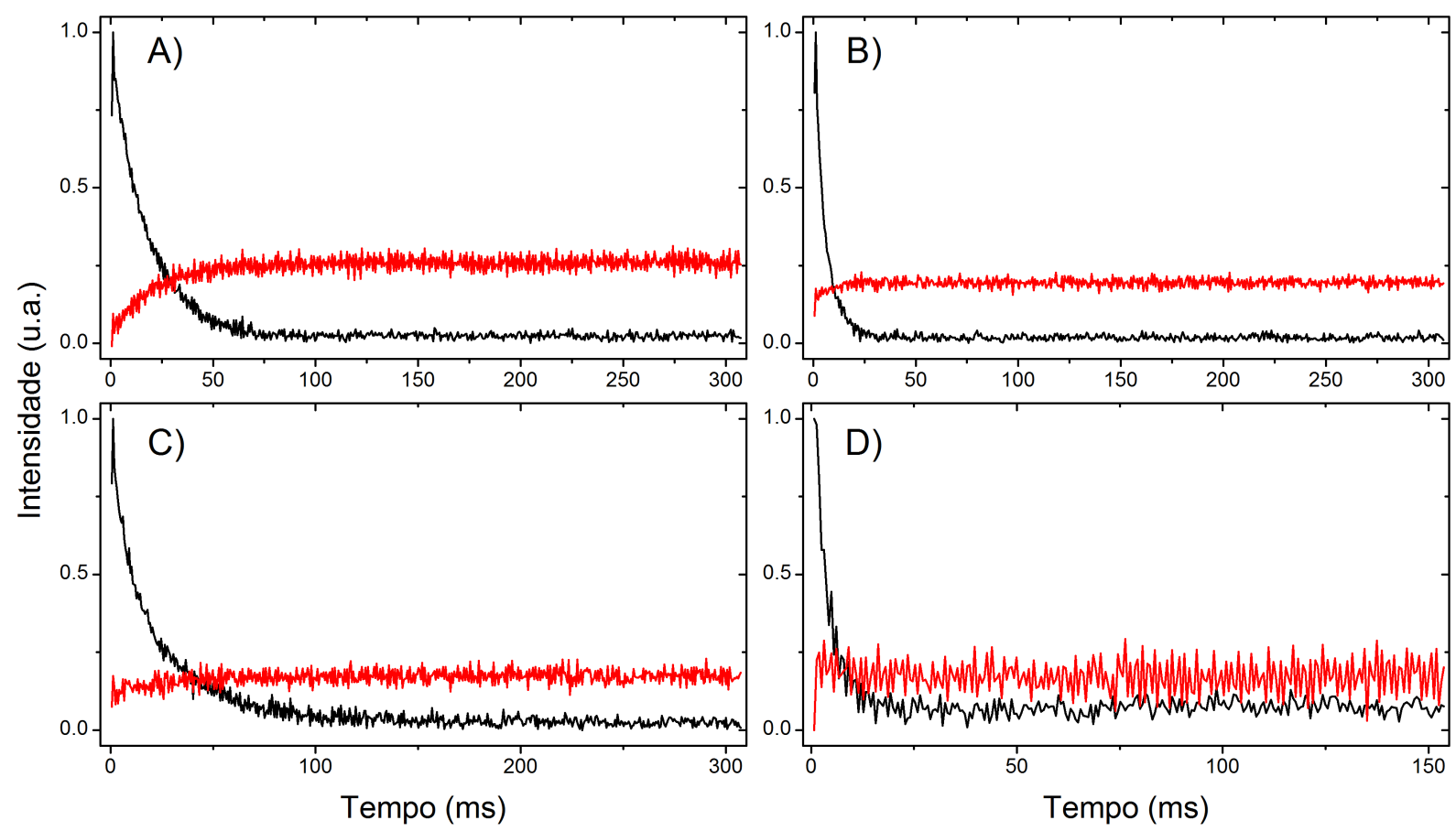

Figura 4.17- Split $180^{\circ}$ anti-simétrica para diferentes razões $T_{1} / T_{2}$, para amostras de água dopada com cobre e três tipos de borracha. Em A) água dopada com cobre com $T_{1} / T_{2}=1$. B) borracha B. com $T_{1} / T_{2}=2,7$, C) borracha C. com $T_{1} / T_{2}=1,9$ e D) borracha P. com $T_{1} / T_{2}=6,7$. Sinais adquiridos com $\tau_{1}=400 \mu \mathrm{s}, \tau_{2}=200 \mu \mathrm{s}, 512$ ecos e 512 médias.

Fonte: Elaborada pelo autor. 
A Fig. 4.18 apresenta sinais Split $180^{\circ}$ adquiridos de dois tipos de queijo Gouda de marcas diferentes. Pela aquisição CPMG determinamos que ambos os queijos apresentam duas componentes exponenciais na relaxação transversal, com A) Queijo 1 com $T_{2}=10 \mathrm{~ms}$ e $54 \mathrm{~ms}$ e B) Queijo 2 com $T_{2}=16 \mathrm{~ms}$ e $51 \mathrm{~ms}$. Os valores de $T_{1}$ são respectivamente $95 \mathrm{~ms}$ e $100 \mathrm{~ms}$. Um decaimento é observado logo no início dos sinais imaginário em ambos os queijos, isso é relacionado com a presença das componentes curtas de $T_{2}$ que em ambos os casos possuí o dobro de amplitude da componente mais longa. Pouca diferença nas amplitudes no estado estacionário é observada.
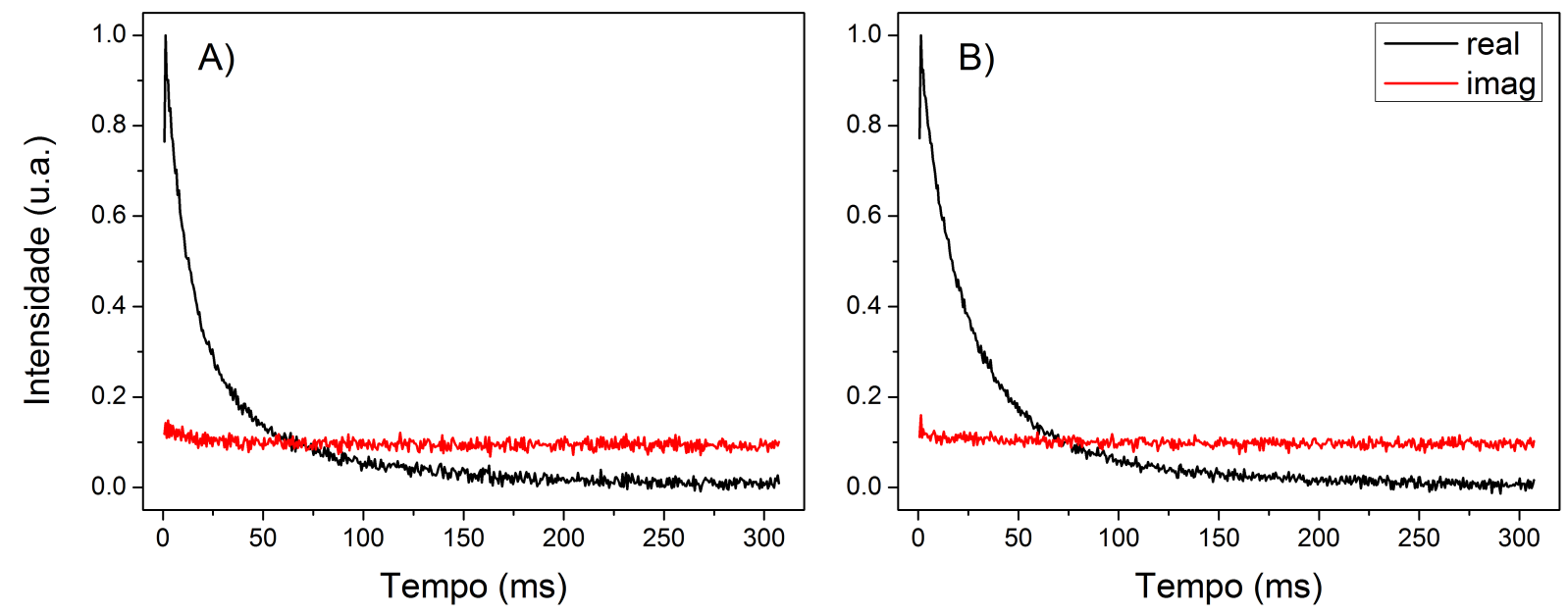

Figura 4.18- Split $180^{\circ}$ anti-simétrica para dois tipos de queijo Gouda, com $\tau_{1}=400 \mu \mathrm{s}, \tau_{2}=200 \mu \mathrm{s}, 512$ ecos e 512 médias. A) com razão $T_{1} / T_{2}=4,3$ e B) e o segundo com $T_{1} / T_{2}=3,3$.

Fonte: Elaborada pelo autor.

\section{Butterfly Pathways}

A fim de explorar as possibilidades e buscar otimizações das sequências Split $180^{\circ}$, realizamos uma série de simulações numéricas para estudar o efeito dos diversos parâmetros da sequência no sinal no estado estacionário, tais como os tempos $\tau_{1}, \tau_{2}$, as fases $\phi_{0}, \phi_{1}$ e $\phi_{2}$ e o offset de frequência.

A Fig. 4.19 apresenta sinais simulados da amplitude do estado estacionário em função do offset de frequência e da razão $\tau_{1} / \tau_{2}$, para a Split $180^{\circ}$ simétrica e anti-simétrica. Como pode ser observado, os máximos e mínimos de amplitude estão distribuídos quase que de maneira opostas em ambas as sequências, principalmente devido a translação do perfil de excitação em sequências com alternância de fase, similar a discussão da seção 2.4.1. As regiões de máximo 
e mínimo possuem complexa dependência com os tempos $\tau_{1}, \tau_{2}$ e o offset de frequência.
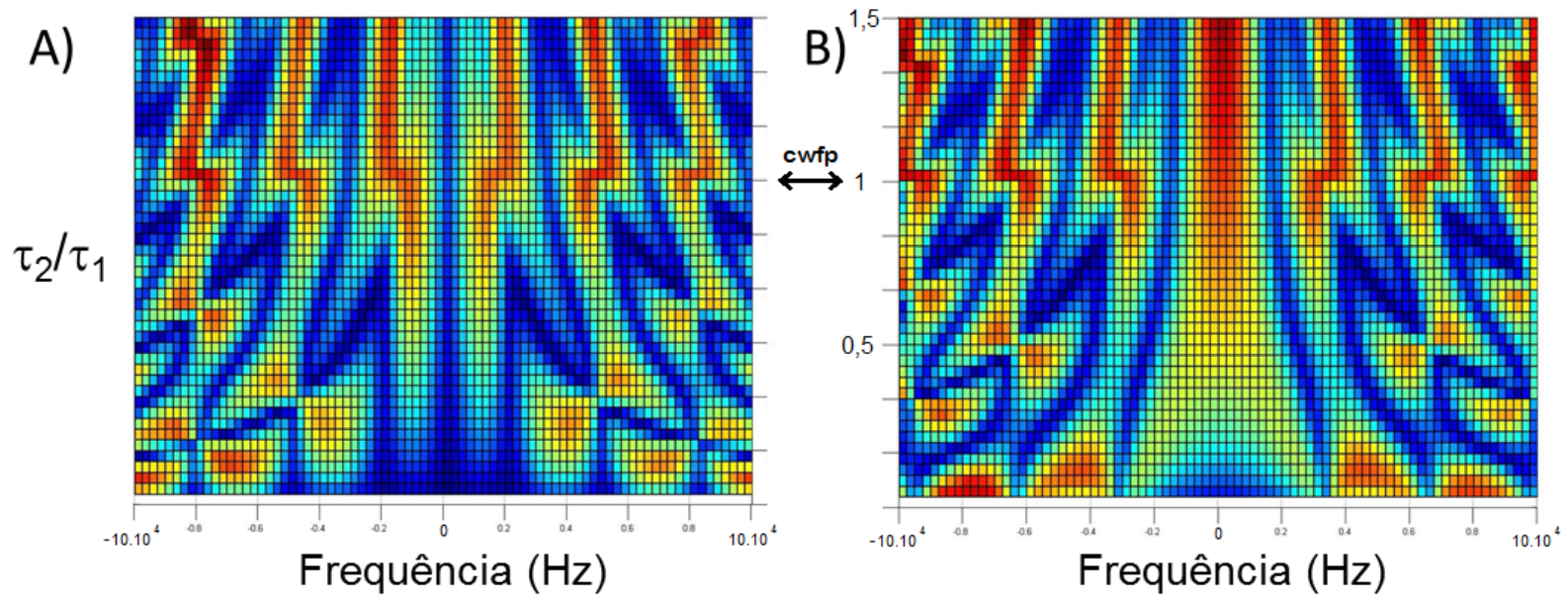

Figura 4.19- Simulação numérica da amplitude dos ecos no estado estacionário em função da razão $\tau_{2} / \tau_{1}$ e do offset de frequência para A) Split $180^{\circ}$ Simétrica $\phi_{1}=\phi_{2}$ e B) Split $180^{\circ}$ Anti-Simétrica $\phi_{1}=-\phi_{2}$.

Fonte: Elaborada pelo autor.

Uma característica interessante da Split $180^{\circ}$ é que na condição $\tau_{1}=\tau_{2}$, ela é equivalente a sequência CWFP, em destaque na Fig. 4.19 com seta, apresentando mesmo perfil de excitação descrito em Fig. 4.1. Assim, a sequência CWFP pode ser vista como um caso específico da geral Split $180^{\circ}$, porém com procedimentos distintos de análises uma vez que uma utiliza o tempo de decaimento ao estado estacionário e a outra foca na amplitude dos ecos.

Um segundo aspecto a ser explorado na otimização das sequências Split $180^{\circ}$ é analisar a formação dos ecos dentro da janela de aquisição durante o período $\tau_{1}$ da sequência. Devido a separação $\tau_{2}$ entre os pulsos de $90^{\circ}$ e a aplicação periódica da sequência, uma série de ecos são formados na janela de aquisição, sendo suas posição definidas pelos tempos $\tau_{1}$ e $\tau_{2}$ e o sinal das amplitudes pelas fases $\phi_{0}, \phi_{1}$ e $\phi_{2}$.

A Fig. 4.20 apresenta ecos adquiridos no MOUSE-NMR para amostra de água dopada com cobre $T_{1}=T_{2}=23 \mathrm{~ms}$, fixando $\tau_{1}=400 \mu \mathrm{s}$ para oito valores de $\tau_{2}$ igualmente espaçados entre 80 e $220 \mu \mathrm{s}$. Apresentamos os ecos adquiridos com a A) Split $180^{\circ}$ simétrica e em B) Split $180^{\circ}$ anti-simétrica. No primeiro caso, vemos a presença de duas componentes ecos de maior amplitude, sendo uma com amplitude negativa e a outra positiva. Para a Split $180^{\circ}$ anti-simétrica ambos os ecos apresentam mesma fase. Alguns ecos de ordem superior e menor amplitude podem ser observados com fases opostas em A) e mesma fases em B) como sugerido nas simulações, Fig. 4.13 e 4.14. 

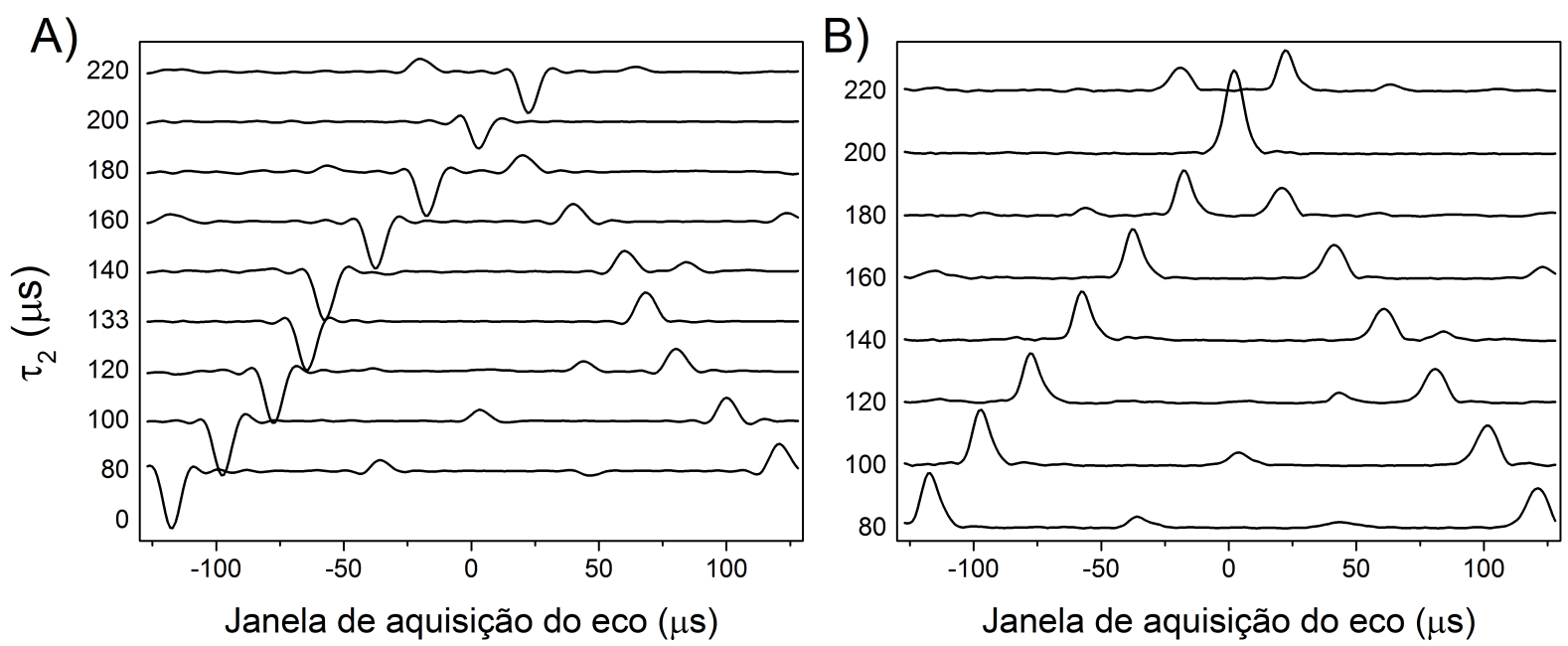

Figura 4.20- Ecos no estado estacionário adquiridos no MOUSE-NMR no intervalo $\tau_{1}$ para as sequências A) Split $180^{\circ}$ simétrica e B) Split $180^{\circ}$ anti-simétrica. Foi utilizada água dopada com cobre $T_{1}=T_{2}=23 \mathrm{~ms}, \tau_{1}=400 \mu \mathrm{s}$ e $\tau_{2}$ entre 80 e $220 \mu \mathrm{s}$.

Fonte: Elaborada pelo autor.

É interessante notar que conforme $\tau_{2}$ cresce em ambos os casos os ecos se aproximam, até a situação em $\tau_{2}=200 \mu \mathrm{s}$, onde os ecos se encontram. Esse ponto é a condição $\tau_{1}=2 \tau_{2} \mathrm{e}$ no caso Fig. 4.20 A) os ecos interferem destrutivamente e em B) construtivamente.

Na Fig. 4.21 apresentamos simulações numéricas do experimento realizado em Fig. 4.20, apresentando a amplitude dos sinais em escala de cor. Azul é positivo e vermelho negativo. Na Fig. $4.21 \mathrm{~A}$ ) para a Split $180^{\circ}$ simétrica e em B) para Split $180^{\circ}$ anti-simétrica.

D. Freed et al. associaram esses padrões dos ecos na Split $180^{\circ}$ com o perfil Hofstadter Butterfly descrito nas referências ${ }^{80,81}$, denominando assim no caso da Split $180^{\circ}$ de 'Butterfly Pathways'. Esse padrão fractal é gerado nas complexas sobreposições dos múltiplos ecos formados nas sequências de multi-pulsos tipo Split $180^{\circ} .{ }^{36}$

Para construir o perfil da Fig. 4.21 experimentalmente com boa resolução, realizamos uma série de experimentos utilizando água dopada com cobre, com $\tau_{1}=400 \mu$ s e $\tau_{2}$ variado entre 40 e $400 \mu \mathrm{s}$ em 144 passos, onde cada linha de valor $\tau_{2}$ foi adquirida com 512 ecos e 1024 médias no caso simétrico e anti-simétrico. O resultado experimental é apresentado na Fig. 4.22 e concorda muito bem com o simulado. 

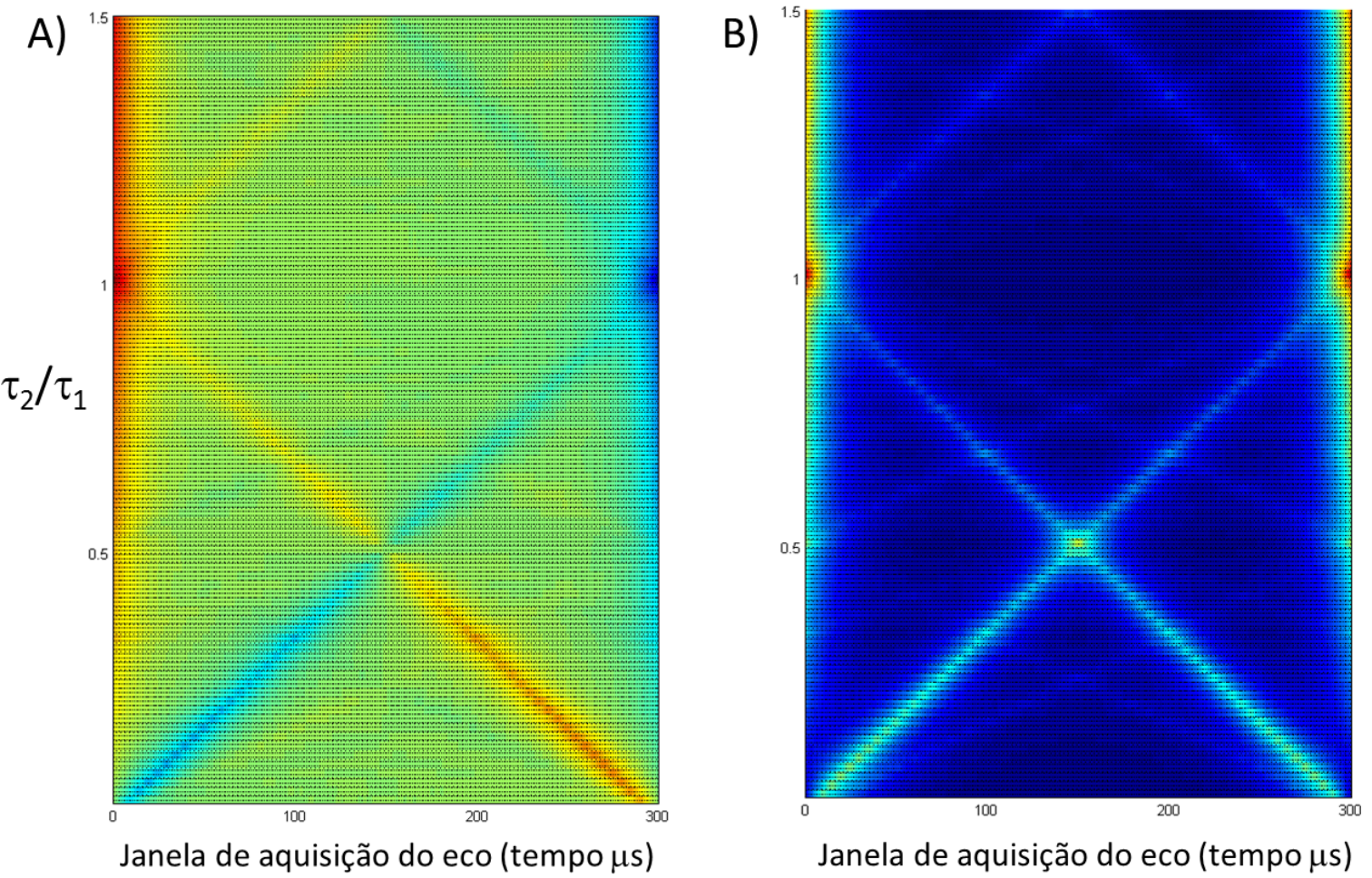

Figura 4.21- Simulação numérica do perfil 'Butterfly' das sequências Split $180^{\circ}$ A) simétrica e B) antisimétrica. Para $F W H M=20 \mathrm{KHz}, \tau_{1}=300 \mu \mathrm{s}$ e $\tau_{2}$ variado entre 10 e $450 \mu \mathrm{s}$.

Fonte: Elaborada pelo autor.

\subsubsection{DECPMG}

Recentemente M. Hürlimann et al. propuseram um método rápido para determinação de mapas de correlação de $T_{1}-T_{2}$, chamado Driven-Equilibrium CPMG (DECPMG). ${ }^{35} \mathrm{~A}$ descrição teórica da técnica foi introduzida na seção 2.5 .3 e aqui apresentaremos resultados obtidos em sua implementação no espectrômetro KEA com sinais adquiridos no magneto mini-Halbach. Os resultados do método DECPMG são comparados com o método convencional para determinação dos mapas de correlação $T_{1}-T_{2}$ através do experimento bi-dimensional introduzido por Song. ${ }^{83}$, descrito na Fig. 2.15.

As Fig. 4.23, 4.24 e 4.25 apresentam a comparação entre mapas de correlação $T_{1}-T_{2}$ obtidos com o experimento unidimensional DECPMG e com o método bi-dimensional, para as amostras de borracha em água, queijo D. (duro) misturado com leite e queijo M. (mole) com leite. Todos os mapa bi-dimensionais adquiridos com a sequência de pulsos convencional $T_{1}-T_{2}$ foram adquiridos com lista de $\tau$ com 32 experimentos, usando $\tau_{\text {cpmg }}=400 \mu \mathrm{s}, 2048$ ecos e 4 médias totalizando 2,5 minutos de tempos experimental. Esses sinais bi-dimensionais 


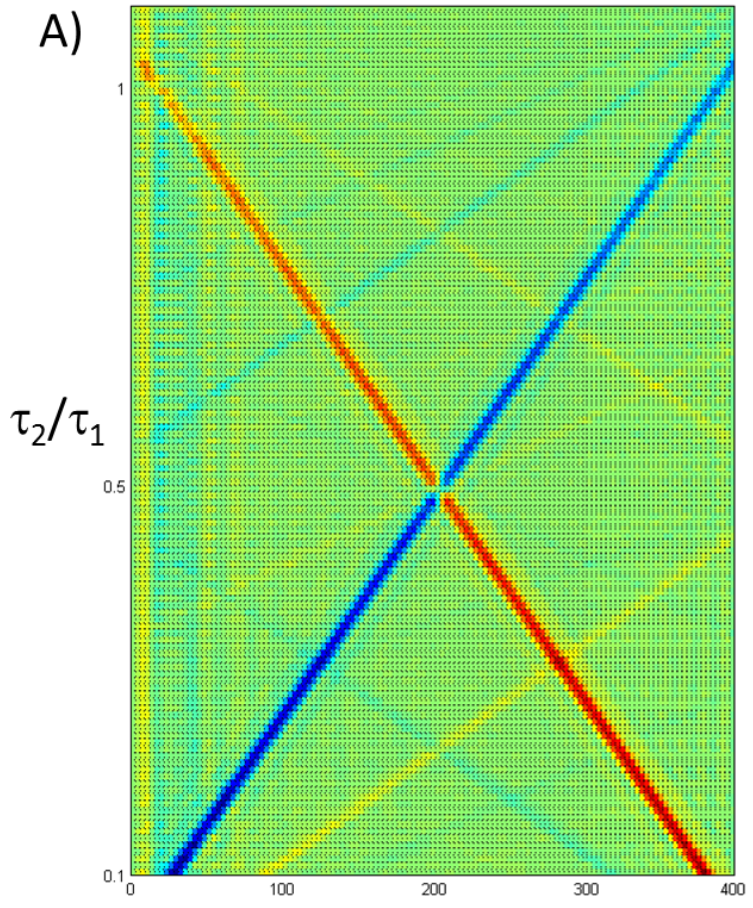

Janela de aquisição do eco (tempo $\mu$ s)
B)

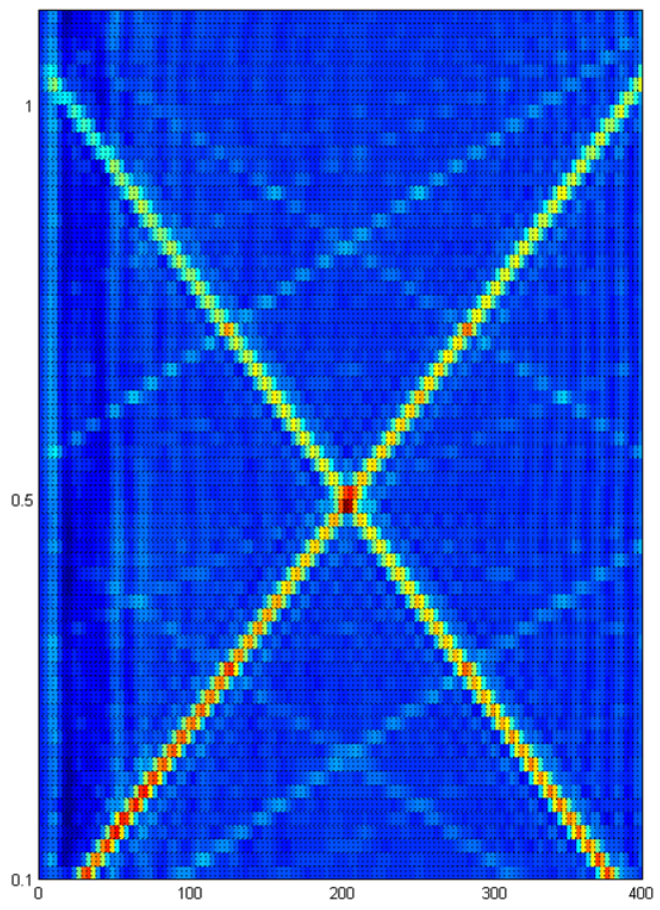

Janela de aquisição do eco (tempo $\mu$ s)

Figura 4.22- Perfil 'Butterfly' experimental das sequências Split $180^{\circ}$ adquiridos no MOUSE-NMR para água dopada com cobre $T_{1}=T_{2}=23 \mathrm{~ms}$. Em A) Split $180^{\circ}$ simétrica e B) Split $180^{\circ}$ anti-simétrica. Com 512 ecos, 1024 médias e $\tau_{1}=400 \mu \mathrm{s}$ e $\tau_{2}$ variado entre 40 e $400 \mu \mathrm{s}$ em 144 passos.

Fonte: Elaborada pelo autor.

foram processados com a Transformada Inversa de Laplace 2D.

Para os experimentos DECPMG foram utilizados $\tau_{\text {decpmg }}=400 \mu \mathrm{s}, \tau_{1}=400 \mu \mathrm{s}, \tau_{2}=300$ $\mu \mathrm{s}, 1000$ ecos em DE, 2048 ecos no bloco cpmg-like e 4 médias, com tempo experimental total de 10 segundos. O processamento dos sinais unidimensionais na obtenção dos mapas abaixo foi realizado como descrito na referência. ${ }^{35}$

O primeiro resultado que apresentamos é a situação mais simples onde temos duas componentes com tempos de relaxação muito distintos, como é o caso de um pedaço de borracha imerso em água dopada com cobre. Na Fig. 4.23 A) e B) comparamos os resultado obtidos com o experimento A) $T_{1}-T_{2}$ convencional e B) adquirido com a DECPMG. O experimento $T_{1}-T_{2}$ convencional teve duração total de 2,5 minutos e o experimento DECPMG 10 segundos.

Apesar da DECPMG não definir uma região simétrica no mapa de distribuição, sua estimativa se apresenta muito satisfatória. Note que na Fig. 4.23 B) o pico com tempo mais curto apresenta ponto de máxima intensidade no exato centro da distribuição de tempos mais curto no mapa convencional em $A$ ). 

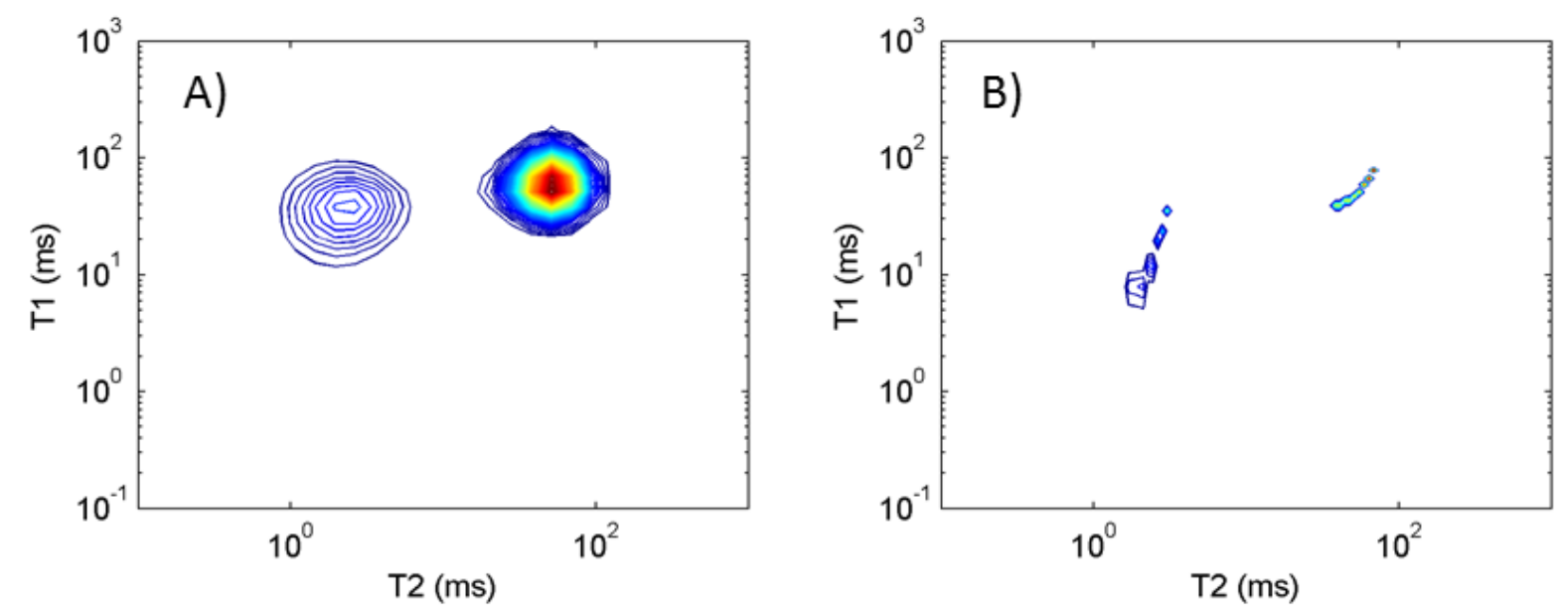

Figura 4.23- Comparação dos mapas de distribuição $T_{1}-T_{2}$ do conjunto água dopada e borracha, para as sequências de pulsos A) $T_{1}-T_{2}$ bi-dimensional com tempo total de aquisição 2,5 minutos e B) DECPMG unidimensional, com tempo de aquisição de 10 segundos.

Fonte: Elaborada pelo autor.

Na Fig. 4.24 apresentamos a comparação dos resultados de cada sequência de pulso para o conjunto queijo D. e leite. Temos que os resultados concordam novamente onde o tempos de $T_{1}$ próximo de 100 ms são associados ao leite e acima de 200 ms ao queijo.
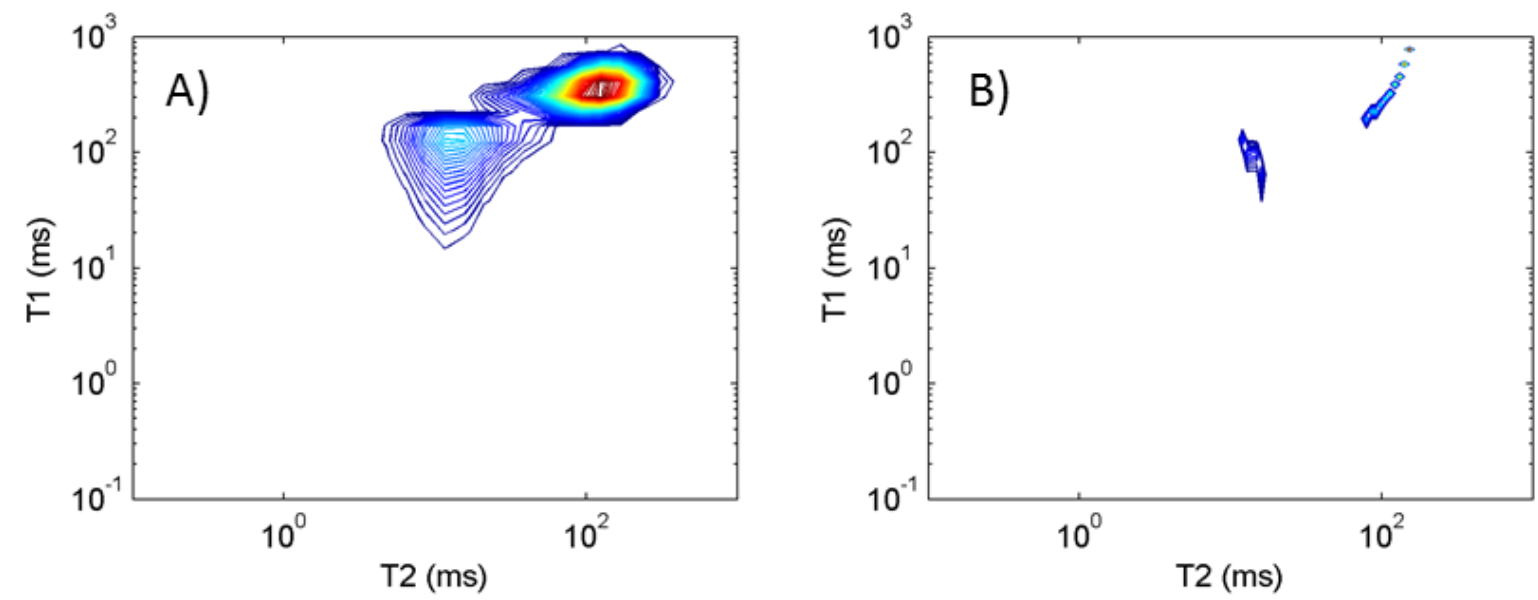

Figura 4.24- Comparação dos mapas de distribuição $T_{1}-T_{2}$ do conjunto queijo $D$. e leite, para as sequências de pulsos A) $T_{1}-T_{2}$ bi-dimensional e B) DECPMG unidimensional.

Fonte: Elaborada pelo autor.

Na Fig. 4.25 outro tipo de queijo mais mole que o primeiro é misturado com leite. Temos que as componentes do queijo M. e do leite estão praticamente sobrepostas, apresentando uma grande distribuição de tempos na faixa de $T_{1}$ entre 100 e 500 ms e para $T_{2}$ entre 20 e 200 ms. O resultado obtido com a DECPMG concorda muito bem com o experimento convencional.

O método DECPMG potencialmente extrai mapas $T_{1}-T_{2}$ em apenas duas aquisições, 

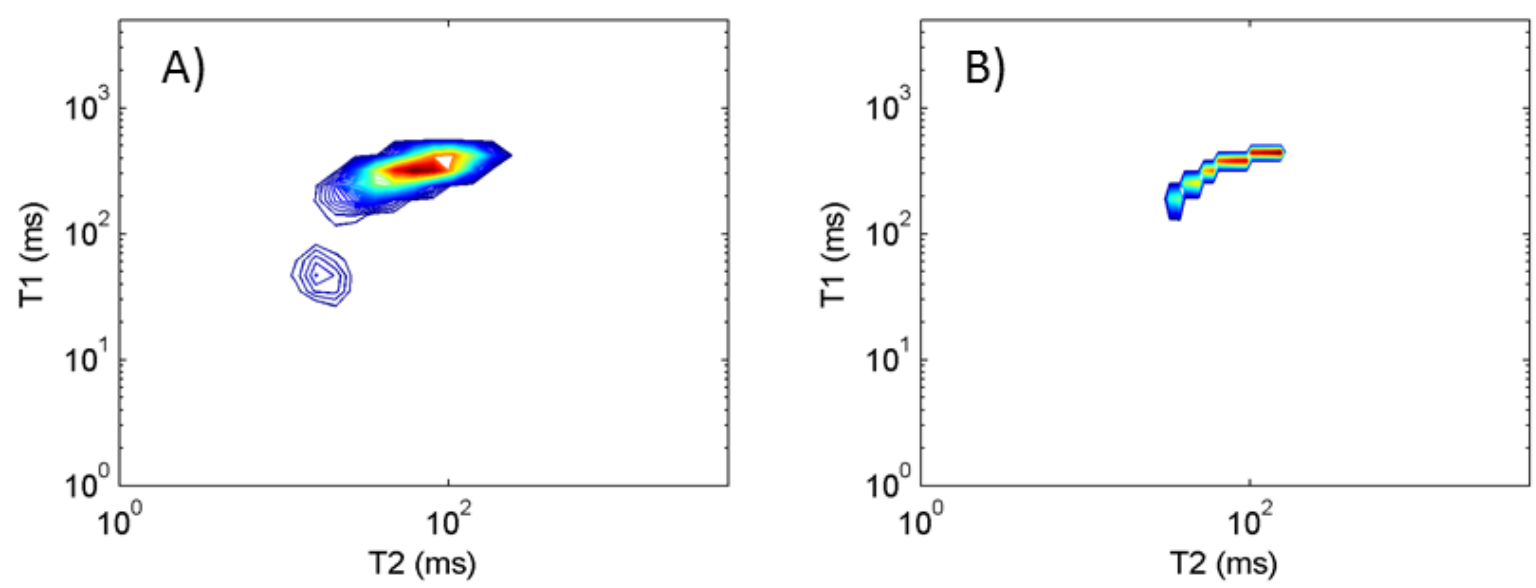

Figura 4.25- Comparação dos mapas de distribuição $T_{1}-T_{2}$ do conjunto queijo $\mathrm{M}$. e leite, para as sequências de pulsos A) $T_{1}-T_{2}$ bi-dimensional e B) DECPMG unidimensional.

Fonte: Elaborada pelo autor.

com uma aquisição DECPMG e uma CPMG. É importante notar que o método DECPMG é diferente de outros driven equilibrium métodos ${ }^{35}$, pois não apresenta a limitação de amostras heterogêneas, com larga distribuição de tempos de relaxação e é sensível a uma faixa de tempos de relaxação longa, permitindo a extração das informações $T_{1}$ e $T_{2}$ em apenas duas aquisições. Note também que o processamento dos sinais DECPMG na contrução dos mapas $T_{1}-T_{2}$ não envolvem a transformada inversa de laplace $2 \mathrm{D}$, mas apenas a unidimensional.

A rapidez e robustez da DECPMG para determinação rápida de mapas bi-dimensionais $T_{1}-T_{2}$ apresenta-a com uma forte candidata na análise de rotinas em aplicações industriais.

\subsubsection{Driven-Equilibrium modificada}

Nesta seção apresentamos uma variação da sequência Driven-Equilibrium CPMG que trabaIhamos durante o intercâmbio na RWTH Aachen University. A modificação é baseada na transformação do intervalo $\tau_{2}$ em uma variável para assim possibilitar a determinação da razão $T_{1} / T_{2}$ em uma única aquisição rápida.

Implementamos a sequência DECPMG onde $\tau_{2}$ passa a ser uma variável $\tau_{2}(i)$ com $i$ componentes de uma lista. A Fig. 4.26 apresenta um esquema da sequência proposta, com pulso inicial de $90^{\circ}$ seguido de dois pulsos de refocalização de $180^{\circ}$ e um segundo pulso de $90^{\circ}$ no ponto central da formação do segundo eco. Em um regime rápido de pulsos, com 
$\tau_{1}, \tau_{2}<<T_{1}, T_{2}$, esse procedimento atinge um estado estacionário na magnetização com amplitude dependente das razões $\tau_{1} / \tau_{2}$ e $T_{1} / T_{2}$.

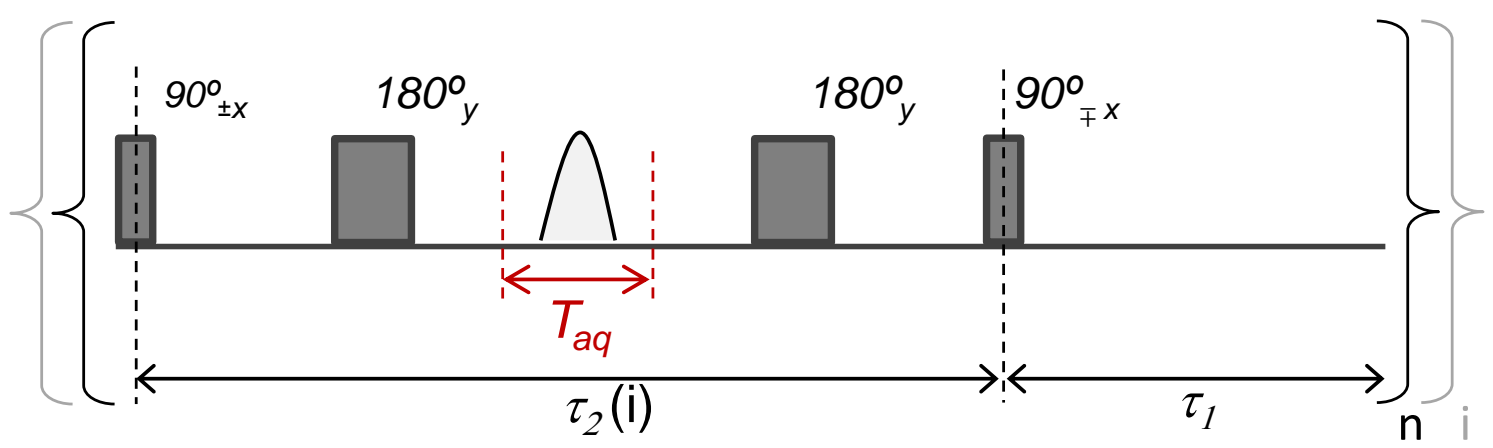

Figura 4.26- Sequência de pulsos Driven-Equilibrium modificada, onde o intervalo $\tau_{2}$ passa a ser uma variável $\tau_{2}(i)$ com $i$ componentes. Repetindo esse bloco de pulsos $n$ vezes a magnetização atinge um estado estacionário dependente das razões $\tau_{1} / \tau_{2}$ e $T_{1} / T_{2}$.

Fonte: Elaborada pelo autor.

Pela equação 2.5 .13 , a modificação é descrita com

$$
M_{d e}=M_{0} \frac{1}{1+\frac{\tau_{2}(i) T_{1}}{\tau_{1} T_{2}}}
$$

A proposta da sequência é que a cada $n$ repetições do bloco de pulsos da Fig. 4.26, a lista de $\tau_{2}(i)$ passa para seu próximo valor, ocorrendo assim uma mudança na amplitude do estado estacionário, que se monitorado resulta em decaimentos similares aos sinais simulados apresentados na Fig. $4.27 \mathrm{~A}$ ) para $T_{1} / T_{2}=1,1,3$ e 2 .

Na Fig. 4.27 B) apresentamos sinais experimentais adquiridos de uma amostra de água dopada com cobre, apresentando toda a transição da magnitude dos ecos de um estado estacionário ao próximo, em função do parâmetros $\tau_{2}(i) / \tau_{1}$. Através de fitting numérico das curvas obtidas similares a Fig. 4.27 B), os valores da razão dos tempos de relaxação são obtidos $T_{1} / T_{2}$.

Na Fig. 4.28 apresentamos resultados experimentais obtidos da Driven-Equilibrium modificada comparados com os valores medidos pelas sequências convencionais cpmg e inversão recuperação. Em A) para quatro tipos de polímeros pec $2 k$, pec4k, pec6k e pec8k, com diferentes razões $T_{1} / T_{2}$, medidos em triplicata à $24^{\circ} \mathrm{C}$ com $\tau_{1}=400 \mu \mathrm{s}$ e $\tau_{2}=100$ até $1900 \mu \mathrm{s}$. Em B) comparação das razões $T_{1} / T_{2}$ dos óleos comestíveis Azeite de Oliva, Colza (Rapessel), Gergelim (Sesame) e linhaça (Kunella Lein), medidos em decuplicata (10 vezes) à $24^{\circ} \mathrm{C}$ com 

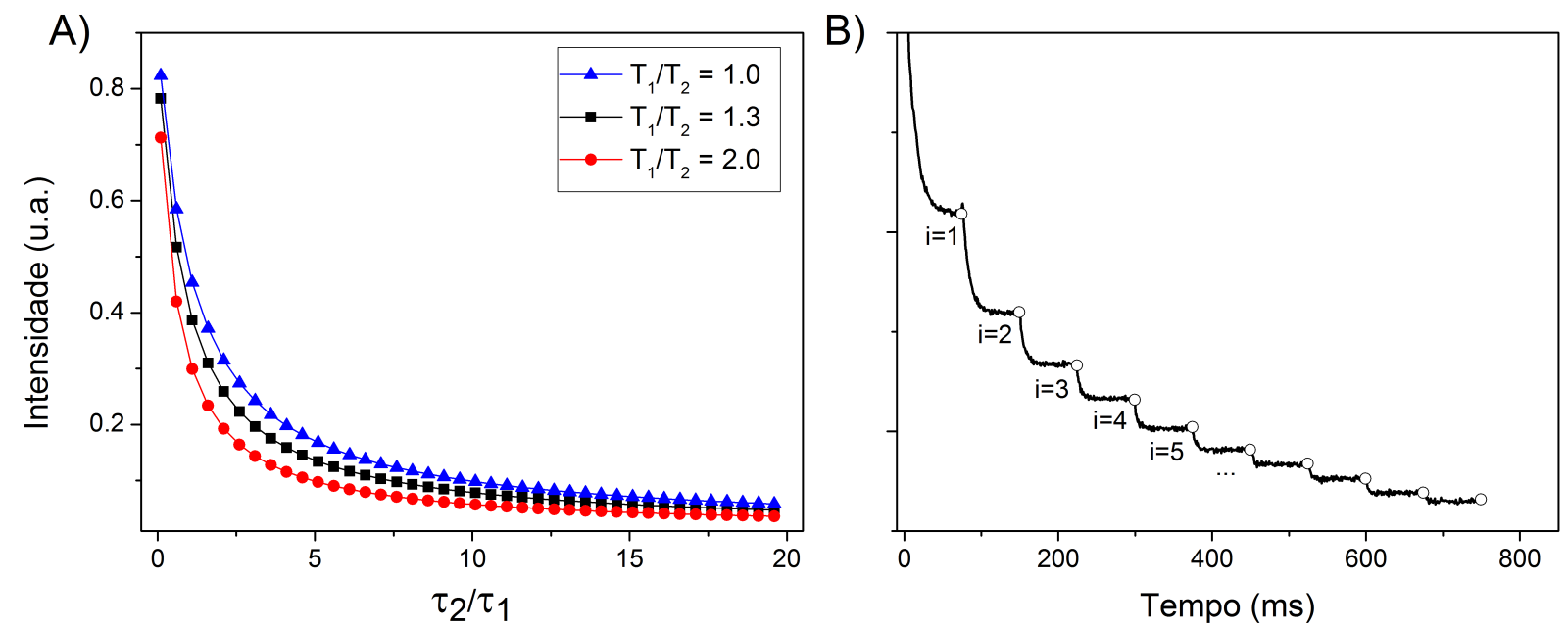

Figura 4.27- Em A) sinais simulados da sequência Driven-Equilibrium modificada com a amplitude em função da lista $\tau_{2}(i) / \tau_{1}$, para $T_{1} / T_{2}=1,1,3$ e 2 . B) apresenta sinal experimental apresentando o comportamento da magnetização na passagem de um estado estacionário ao próximo, onde $i$ denota a transição da lista $\tau_{2}(i) / \tau_{1}$.

$\tau_{1}=500 \mu \mathrm{s}$ e $\tau_{2}=100$ até $3200 \mu \mathrm{s}$.
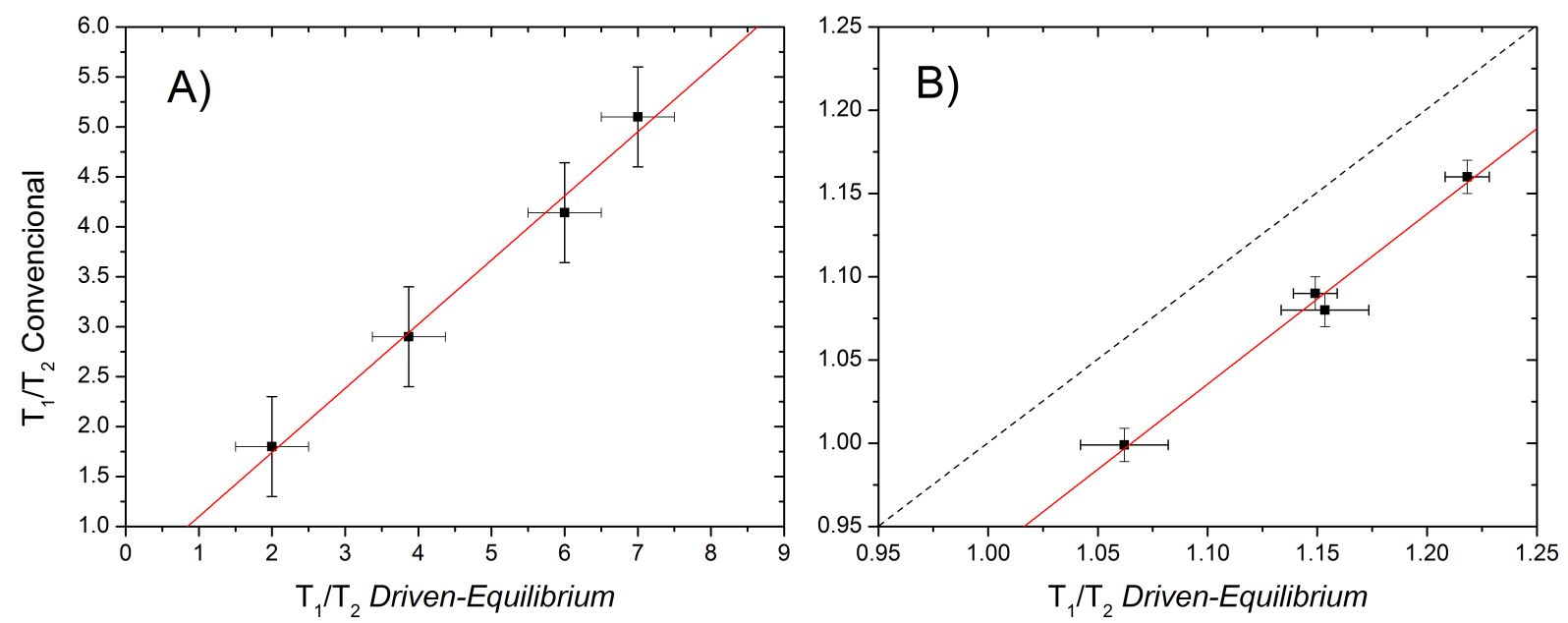

Figura 4.28- Comparação das razões $T_{1} / T_{2}$ obtidas com as sequências convencionais cpmg e inversão recuperação com a Driven-Equilibrium modificada. Em A) para polímeros pec $2 k$, pec $4 k$, pec6k e pec8k em triplicata e em B) para óleos comestíveis Azeite de Oliva, Colza, Gergelim e Linhaça em decuplicata.

Fonte: Elaborada pelo autor.

Esses resultados mostram uma boa correlação entre os valores de $T_{1} / T_{2}$ são obtidos pela a sequência proposta, porém uma leve tendência a valores mais elevados de $T_{1} / T_{2}$ é observado. Esse pequeno desvio é associado com a necessidade de se aplicar longos tempos entre pulsos em determinadas situações, de modo que efeitos de difusão torna-se relevantes, gerando $T_{2}$ efetivo mais curto e portanto razão $T_{1} / T_{2}$ elevada. Resultados mostram que o método proposto pode ser interessante na análise rápida em rotina para aplicações industriais e caracterização. 


\section{Capítulo 5}

\section{Estado Estacionário em Alta Resolução}

Neste capítulo apresentamos os resultados e discussões dos métodos SSFP em alta resolução propostos. Vantagens e desvantagens dos novos métodos SSFPdx e SSFPdxdt são comparados com as técnicas convencionais de aquisição.

\subsection{Caracterização experimental da SSFP}

Iniciamos os resultados e discussões da SSFP em alto campo apresentando uma caracterização experimental dessas sequências. Para isso foram utilizadas duas amostras simples, uma que apresenta apenas uma única componente de frequência $\left(\mathrm{H}_{2} \mathrm{O} / \mathrm{D}_{2} \mathrm{O}\right)$ e outra com duas componentes: água e acetona $\left(\mathrm{H}_{2} \mathrm{O}+\mathrm{C}_{3} \mathrm{H}_{6} \mathrm{O} / \mathrm{D}_{2} \mathrm{O}\right)$, possibilitando melhor visualização do comportamento das fases das componentes FID e Eco. Experimentos foram realizados no espectrômetro AVANCE III Bruker, $600 \mathrm{MHz}$ para o hidrogênio.

A Fig. 5.1 apresenta a comparação de sinais de ${ }^{1} \mathrm{H}$ adquiridos da $\mathrm{H}_{2} \mathrm{O} / \mathrm{D}_{2} \mathrm{O}$ em três condições experimentais. Em A) FID adquirido com a sequência de um único pulso de $90^{\circ}(z g)$ com longo tempo de aquisição $T_{a q}=1 \mathrm{~s}$ e de reciclo $d_{1}=1 \mathrm{~s}^{*}$; em B) FID adquirido com mesma sequência de pulsos, entretanto com tempo de aquisição de apenas $30 \mathrm{~ms}$ e tempo de reciclo $d_{1}=1 \mathrm{~s}$; e em C) o FID SSFP com $T_{a q}=29.7 \mathrm{~ms}$ e $d_{1}=0.3 \mathrm{~ms}$, totalizando

\footnotetext{
* Nos softwares da Bruker tempo de reciclo é denominado $d_{1}$
} 
$T_{p}=T_{a q}+d_{1}<<T_{1}$ e $T_{2}$ nessa condição.

No sinal SSFP, Fig. 5.1 C), pode-se observar a baixa amplitude típica do sinal saturado e a presença da componente eco no final do tempo de aquisição. A aquisição do sinal SSFP foi realizada após a aplicação de uma série de pulsos de RF, garantindo assim a aquisição do sinal no estado estacionário.

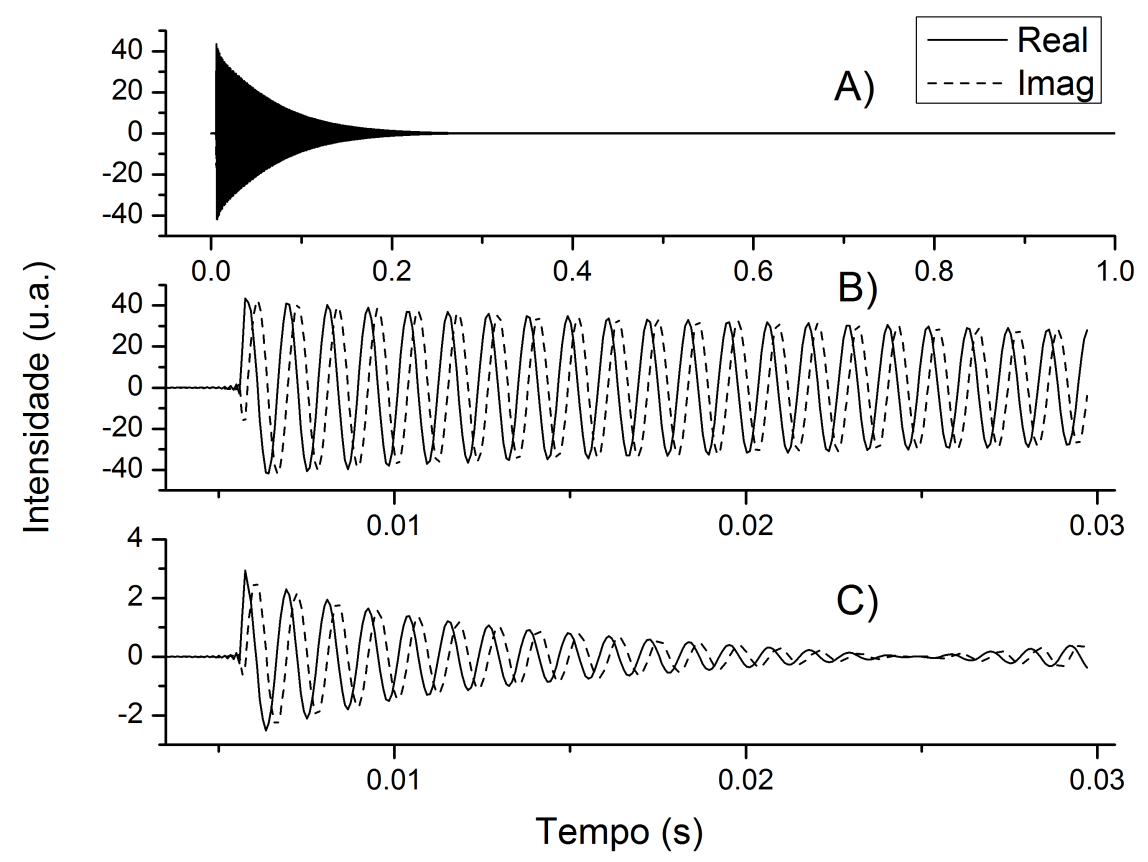

Figura 5.1- Sinais de ${ }^{1} \mathrm{H}\left(\mathrm{H}_{2} \mathrm{O} / \mathrm{D}_{2} \mathrm{O}\right)$ para as sequências A) Pulso simples (zg), B) Pulsos simples truncada em $30 \mathrm{~ms}$, com tempo de reciclo longo e C) SSFP com $T_{a q}=29.7, d_{1}=0.3 \mathrm{~ms}$, todos com pulso de $\theta=90^{\circ}$ e 4 médias.

Fonte: Elaborada pelo autor.

A Fig. 5.2 A) apresenta todo o comportamento da magnetização desde o início da aplicação dos pulsos até a estabilização do estado estacionário. Após um tempo transiente - no intervalo de 0 a 2 s, Fig. 5.2 A) - o sinal entra no estado estacionário mantendo amplitude constante enquanto for continuada a aplicação dos pulsos. Na Fig. 5.2 B) e C) temos a ampliação das regiões no início e final, as regiões sem sinal são as posições de aplicação de pulsos de RF, de aproximadamente $10 \mu s$, não estando em escala real na visualização.

As Fig. 5.1 e 5.2 evidenciam que na SSFP devido a ausência de tempo de reciclo e curto tempo de aquisição, a magnetização não tem tempo de retornar a posição de equilíbrio, forçando-a estabelecer um estado estacionário, que satura o sinal e forma a composição composição FID + Eco. Como discutido na descrição teórica do capítulo 2, o sinal SSFP tem forte dependência com o deslocamento do offset de frequência. A Fig. 5.3 apresenta o sinal no 


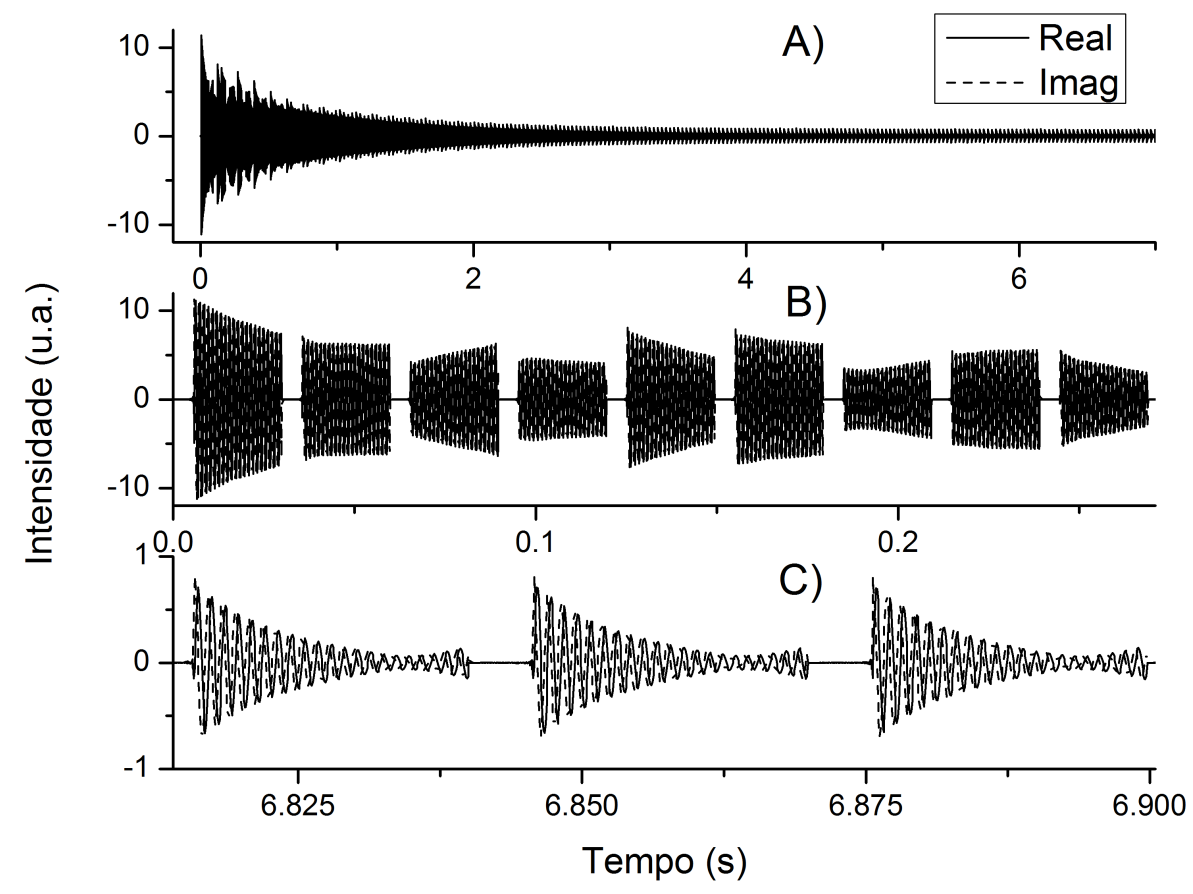

Figura 5.2- A) Apresenta a sequência de FIDs adquiridos na SSFP evidenciando o comportamento da magnetização até estabelecimento do Estado Estacionário após 2 segundos. Em B) e C) temos a ampliação das regiões do início e final, a regiões sem sinal são as posições de aplicação de pulsos de RF. Pulsos de RF tem cerca de $10 \mu$ s de duração e a figura não está em escala.

Fonte: Elaborada pelo autor.

estado estacionário em quatro posições de offset diferentes, em pontos de mínimo e máximo de amplitude. Esses pontos de máximo e mínimo estão de acordo com a supressão periódica de $1 / T_{p}$, onde $T_{p}=30 \mathrm{~ms}$.

A dependência da amplitude da SSFP com a frequência de offset é devido a interação construtiva ou destrutiva das componentes FID e eco em cada situação. A componente eco na SSFP não vem de contribuição única, mas é formada devido a composição de múltiplos ecos formados pela aplicação contínua dos pulsos, como descrito na seção 2.2 .

Para analisarmos as contribuições desse múltiplos ecos na SSFP, realizamos a separação das componentes exponenciais decrescentes $\left(e^{-i \gamma \Omega t}\right)$ e crescente $\left(e^{+i \gamma \Omega t}\right)$ através do processamento pelo Método da Diagonalização Filtrada (seção 6.1), descrito no artigo. ${ }^{51}$ A Fig. 5.4 A) apresenta as componentes decrescente e crescente do sinal SSFP separadas e em B) vemos o sinal original e o resíduo praticamente nulo da diferença entre o mesmo e as componentes encontradas.

Realizamos essa decomposição dos sinais SSFP para várias aquisições diferentes, e tipicamente os resultados apresentam duas componentes FID (decrescente) e duas componentes eco 

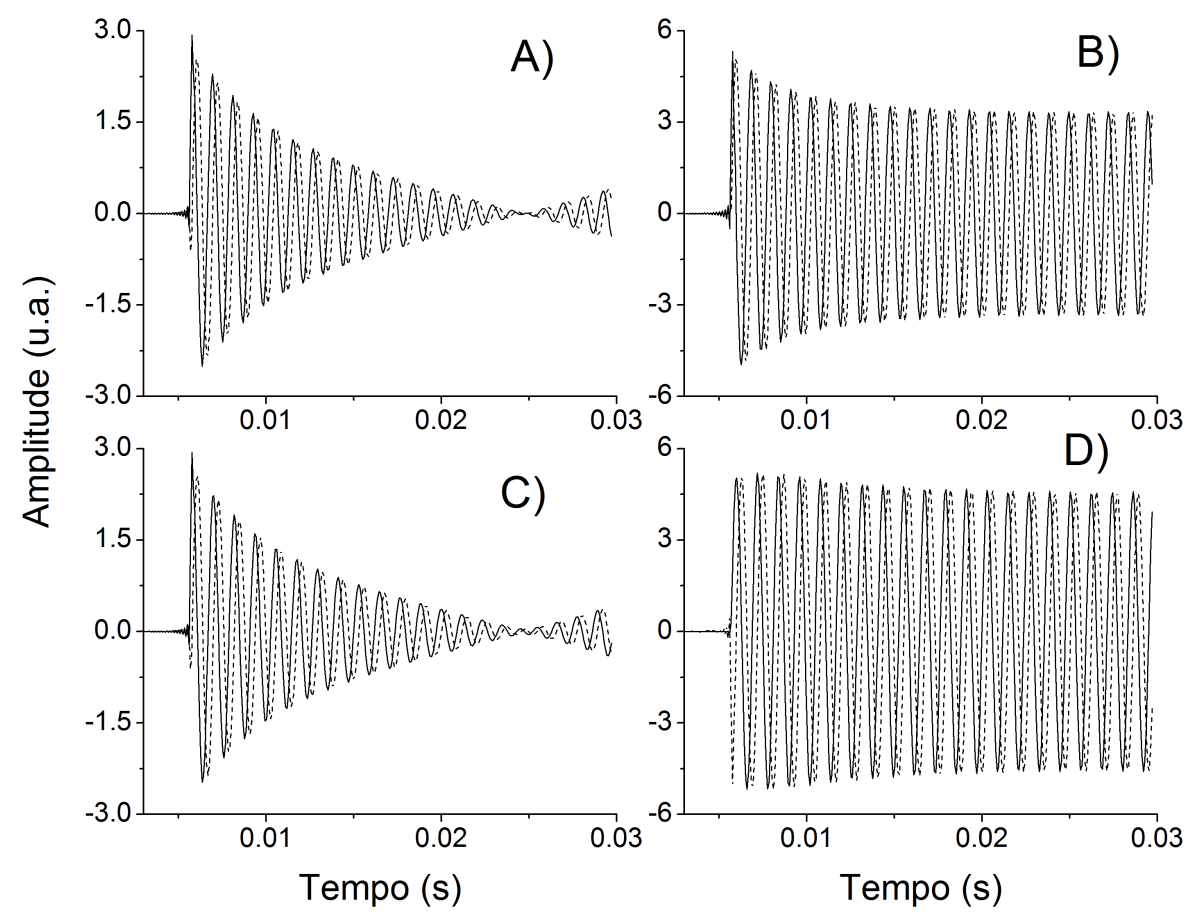

Figura 5.3- FIDs SSFP para quatro valores de offset de frequência: A) 2888 , B) 2904,66 , C) 2954,64 e D) $2938 \mathrm{~Hz}$. Os sinais A) e C) em offset com mínimo de amplitude, onde a componente eco é evidente, e os sinais em B) e D) em posição de máximo de amplitude.

Fonte: Elaborada pelo autor.

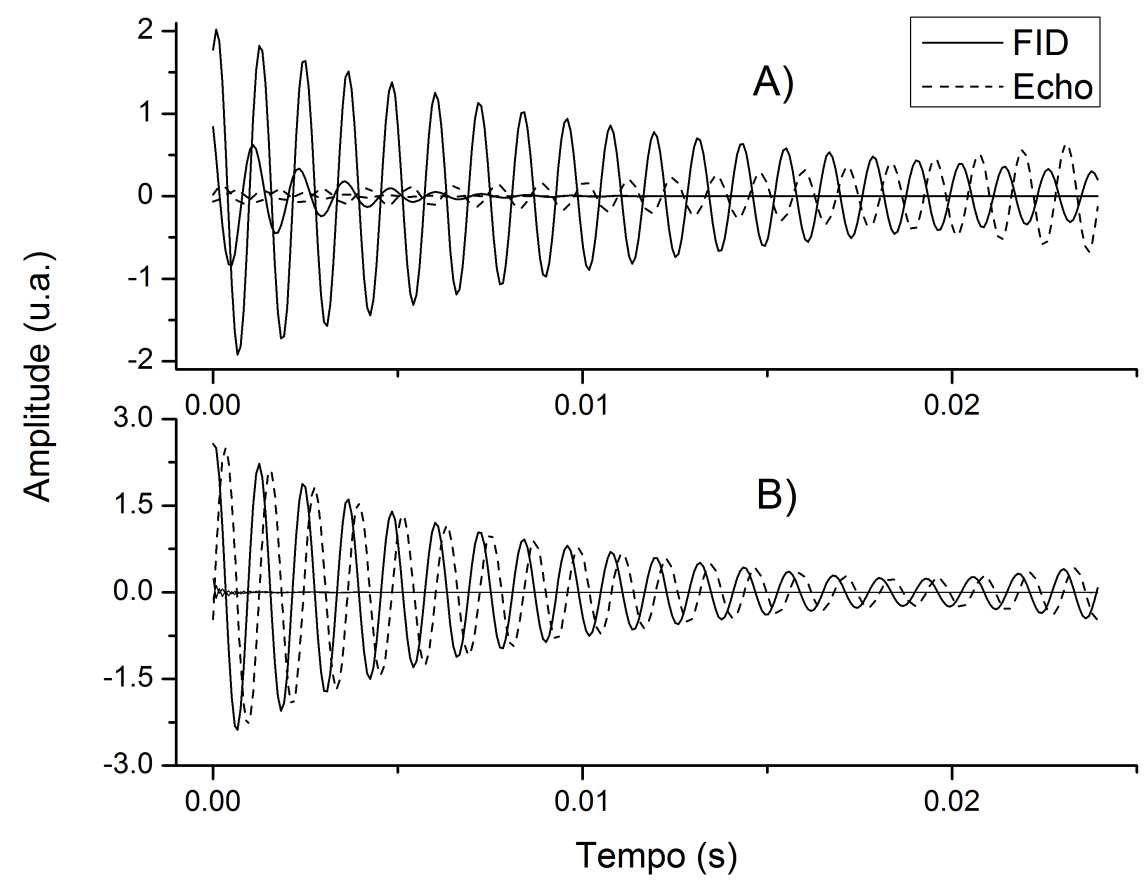

Figura 5.4- A) Componentes FID e eco resultantes da deconvolução do FID-SSFP pelo Método da Diagonalização Filtrada. B) FID original e a linha residual do fitting com o dado experimental.

Fonte: Elaborada pelo autor.

(crescente), sendo um dos ecos de amplitude quase nula. Essas componentes apresentam-se todas em mesma frequência e diferente fases e amplitudes. Essa observação das componentes 
ecos podem ser visualizadas também através da inspeção do sinal SSFP-stopped. ${ }^{53,76}$

O experimento SSFP-stopped é realizado aplicando uma sequência SSFP, porém realizando aquisição do sinal após estabelecer o estado estacionário na magnetização e adquirir a evolução livre da magnetização após cessar a aplicação de pulsos.

A Fig. 5.5 apresenta um sinal SSFP-stopped. Essa aquisição foi realizada após uma SSFP com $T_{p}=100 \mathrm{~ms}$ e pulsos de fase constante. Nota-se que no primeiro período, entre 0 e 0,1 s, o sinal adquirido é idêntico ao acumulado numa sequência SSFP, entretanto, desse ponto em diante, não existem mais os pulsos que mantinham a magnetização no estado estacionário, de modo que a magnetização sofre os processos de relaxação, ainda realizando a refocalização dos diversos ecos advindos dos pulsos previamente aplicados.

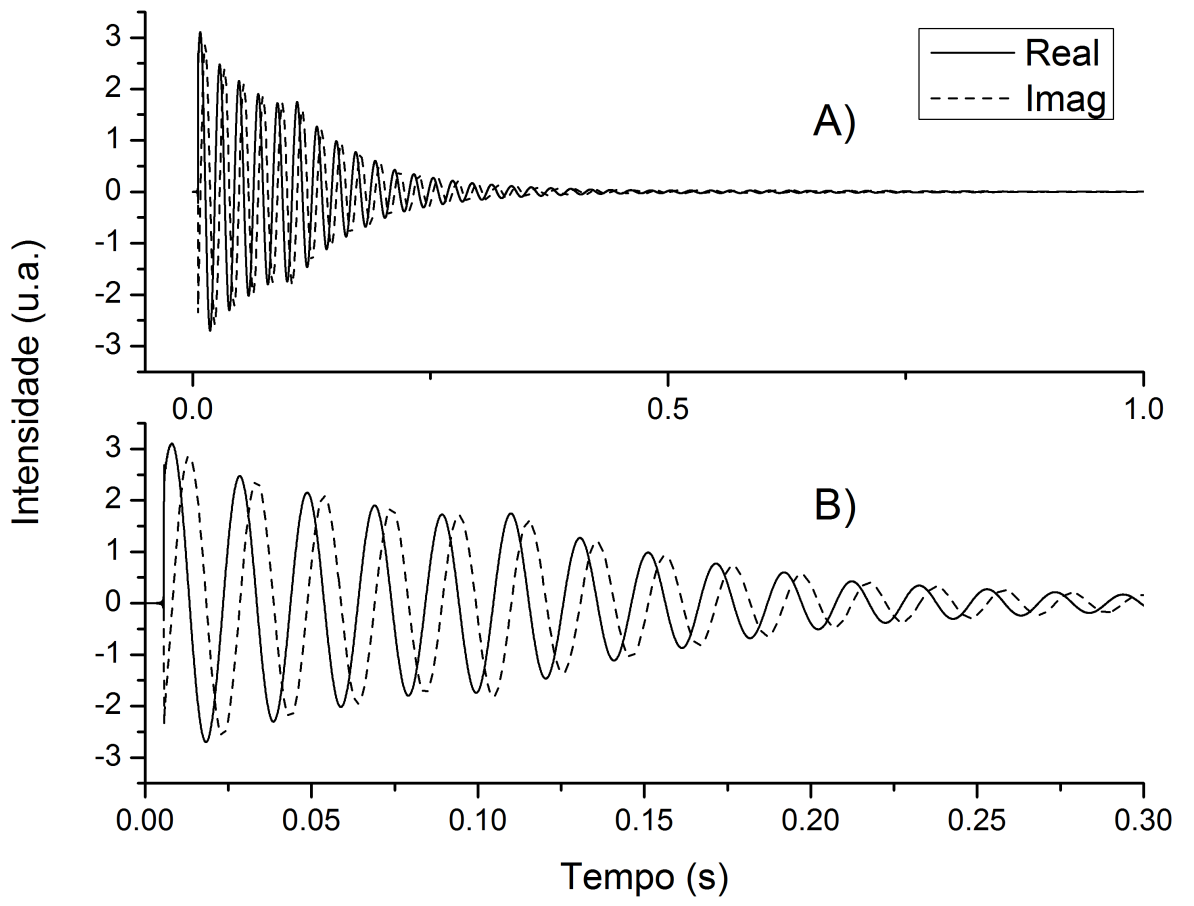

Figura 5.5- Sinal SSFP-stopped adquirida da amostra de $\mathrm{H}_{2} \mathrm{O} / \mathrm{D}_{2} \mathrm{O}$ com $T_{p}=100 \mathrm{~ms}$ e fase constante. Em B) uma ampliação dos três primeiros intervalos entre 0 e 0,3 s.

Fonte: Elaborada pelo autor.

Na descrição teórica do capítulo 2 também discutimos o efeito de controlar a fase dos ecos gerados através das fases dos pulsos na SSFP. A Fig. 5.6 apresenta a comparação dos sinais adquiridos com a sequência SSFP e SSFP com alternância $x$ e -x nos pulsos e detectores. Nota-se que a componente eco observada na SSFP apresenta-se $180^{\circ}$ defasada em relação ao eco da SSFPx-x, tanto na situação de mínimo quanto de máximo de intensidade. Além disso, os offset de frequência que resultam nos máximo de amplitude da SSFP apresenta mínimo de 
intensidade quando adquiridos com a SSFPx-x, como previsto.

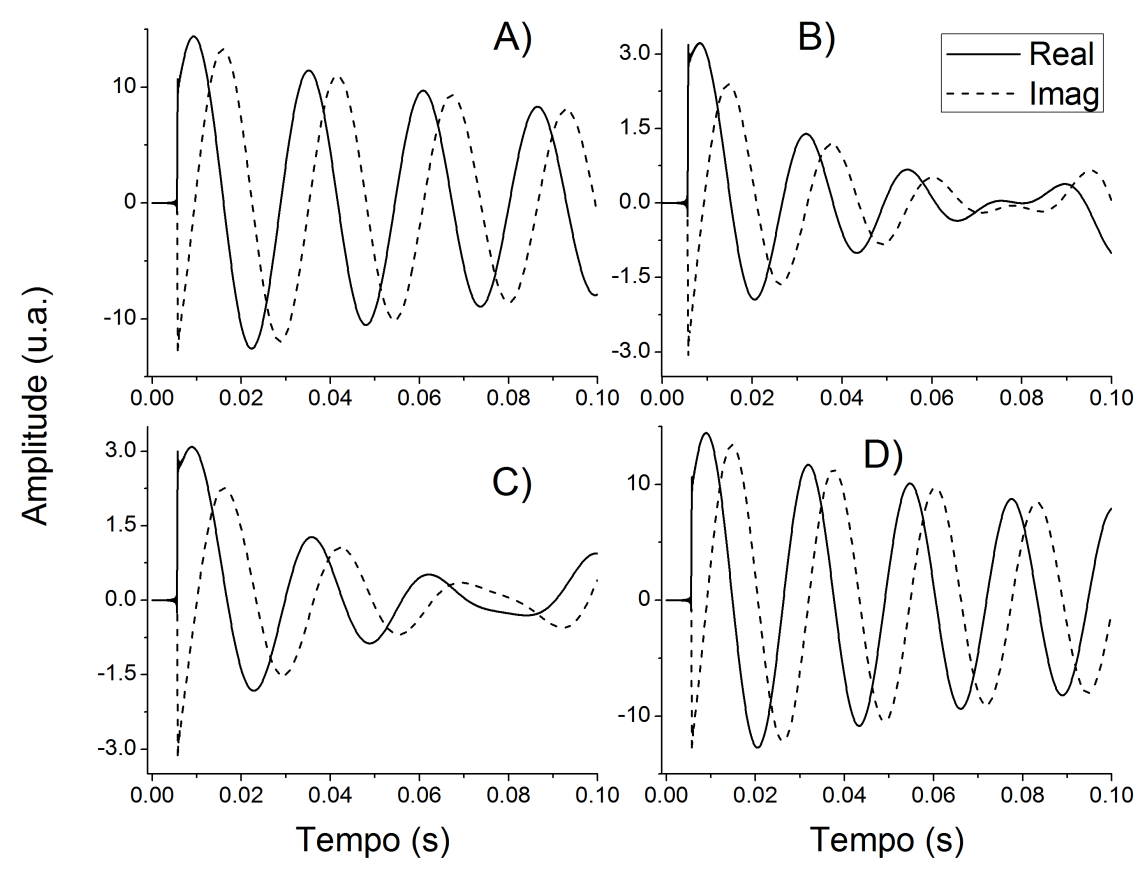

Figura 5.6- A) e B) apresenta os sinal SSFP adquiridos em offset de frequência $2887 \mathrm{~Hz}$ e $2892 \mathrm{~Hz}$ respectivamente, sendo um a situação de máxima amplitude de sinal e a outro mínima. Em C) e D) apresentamos os sinais adquiridos com a sequência SSFPx-x, onde o offset para máximo de sinal é $2892 \mathrm{~Hz}$ e mínimo em $2887 \mathrm{~Hz}$.

Fonte: Elaborada pelo autor.

A dependência dos sinais SSFP com a frequência de offset é melhor visualizada através da construção dos gráficos de perfil de excitação. Esses perfis de excitação foram adquiridos através dos comandos popt no software TopSpin, Bruker. Para o perfil da água foi adquirido 200 espectros, variando a frequência de offset entre 3 e $28 \mathrm{~Hz}$ em passos de 0,125 Hz. No caso do composto de água com acetona foram adquiridos 120 espectros com frequência de offset variando de 0 até $15 \mathrm{~Hz}$ em passos de $0,125 \mathrm{~Hz}$.

A Fig. 5.7 apresenta o perfil de excitação de ${ }^{1} H$ das sequências SSFP em função do ângulo $\theta$ do pulso aplicado para as amostras de $\mathrm{H}_{2} \mathrm{O} / \mathrm{D}_{2} \mathrm{O}$ (à esquerda) e para $\mathrm{H}_{2} \mathrm{O}$ com $\mathrm{C}_{3} \mathrm{H}_{6} \mathrm{O} / \mathrm{D}_{2} \mathrm{O}$ (à direita) para sequências SSFP com diferentes valores de $\theta$ A) $90^{\circ}$, B) $60^{\circ}$, C) $30^{\circ}$ e D) $5^{\circ}$, com $T_{p}=100$ ms. Para melhor destaque das duas componentes do segundo sistema contornamos a modulação da amplitude do pico da água (preto) e da acetona (vermelho) no gráfico à direita.

Esses resultados mostram que as regiões de supressão de sinal são observadas em intervalos periódicos de $1 / T_{p}$ em $\mathrm{Hz}$ e a modulação da amplitude dependente dos tempos de relaxação 

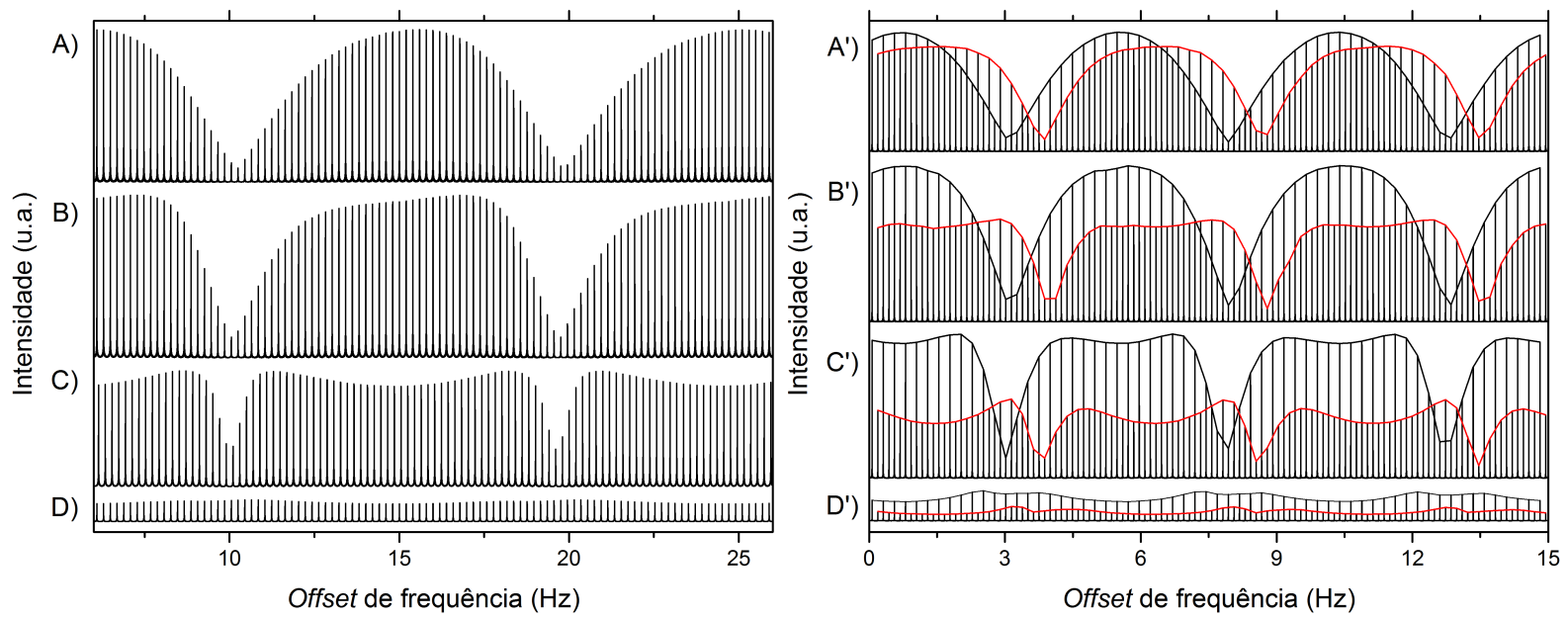

Figura 5.7- Perfil de excitação de ${ }^{1} \mathrm{H}$ para $\mathrm{H}_{2} \mathrm{O} / \mathrm{D}_{2} \mathrm{O}$ (à esquerda) e para $\mathrm{H}_{2} \mathrm{O}$ com $\mathrm{C}_{3} \mathrm{H}_{6} \mathrm{O} / \mathrm{D}_{2} \mathrm{O}$ (à direita) para sequências SSFP com diferentes valores de $\theta$ A) $90^{\circ}$, B) $60^{\circ}$, C) $30^{\circ}$ e D) $5^{\circ}$, com $T_{p}=100$ ms e 4 médias. O contorno vermelho destaca a modulação da amplitude do pico da acetona e o contorno preto da água.

Fonte: Elaborada pelo autor.

$T_{1}$ e $T_{2}$, além do ângulo $\theta$, de acordo com as simulações apresentadas no capítulo 2. Essas modulações de amplitude ao longo do eixo da frequência são indesejáveis em espectros de alta resolução pois informações espectral é perdida nas regiões de supressão.

Uma vez apresentada as principais características dos sinais SSFP, nas próximas seções apresentamos os métodos desenvolvidos para obter uma SSFP com perfil de excitação uniforme, através das SSFP com incremento constante de fase, denominada SSFPdx e da SSFP com incremento não-linear de fase, denominada SSFPdxdt.

\subsection{SSFPdx com incremento linear de fase}

Na proposta inicial desse projeto de pesquisa a exploração das novas sequências de pulsos SSFP com a introdução de alternância de fases para eliminação de anomalias de fase e amplitude nos espectros baseavam-se nos recentes trabalhos que vinham apresentando bons resultados nesse sentido, como T. Rudakov et al. ${ }^{59}$ na Ressonância Quadrupolar Magnética (NQR) e B. Hargreaves et al. ${ }^{55}$ na geração de imagens por Ressonância Magnética (MRI).

Nesta seção apresentamos os avanços obtidos com a SSFP com incremento linear de fase. Na SSFPdx a fase do $n$-enésimo pulso é dada por

$$
\varphi(n)=\varphi_{0}+(n-1) \delta \varphi
$$


onde $n=1,2,3, \ldots, N_{f}$, com $N_{f}$ o último pulso da sequência.

O efeito de introduzir incrementos de fase na SSFP é apresentado na Fig. 5.8 onde em A) temos a SSFP convencional (incremento de fase nulo), os demais com incrementos B) $\pi / 4$, C) $\pi / 2$, D) $3 \pi / 4$, E) $\pi$ (alternância x, -x), F) $5 \pi / 4$, G) $3 \pi / 2$, H) $7 \pi / 4$, adquiridos com $T_{p}=$ $100 \mathrm{~ms}, \theta=90^{\circ}$ e 8 médias.

A) 1

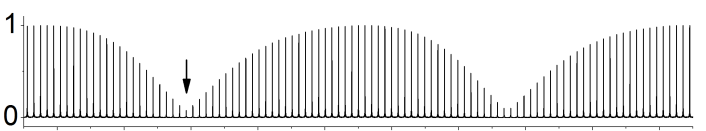

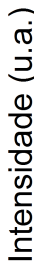

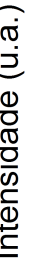

D)

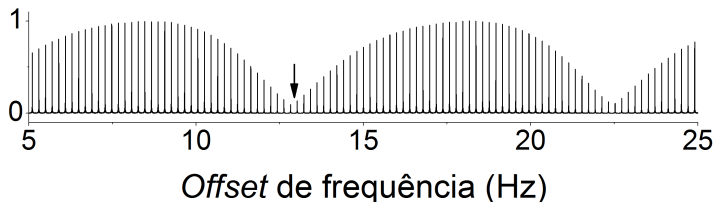

E) 1

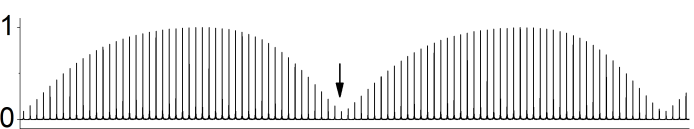

F) 1

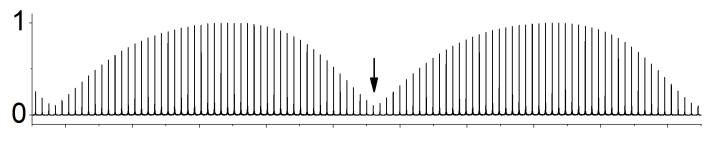

G) 1

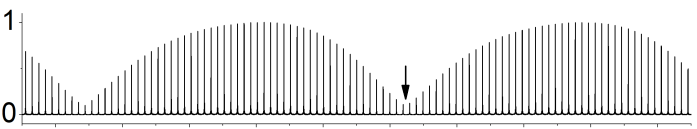

H)

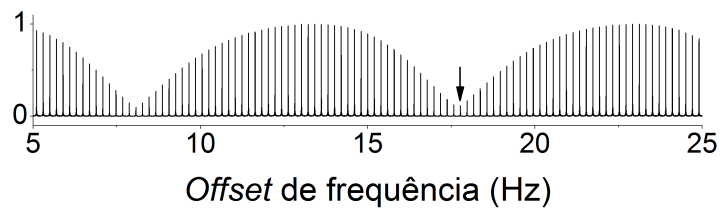

Figura 5.8- Perfil de excitação $\mathrm{H}_{2} \mathrm{O} / \mathrm{D}_{2} \mathrm{O}$ para sequências SSFPdx para diferentes valores de incremento de fase $\delta \varphi$, A) 0, B) $\pi / 4$, C) $\pi / 2$, D) $3 \pi / 4$, E) $\pi$, F) $5 \pi / 4$, G) $3 \pi / 2$, H) $7 \pi / 4$.

Fonte: Elaborada pelo autor.

As setas indicam o deslocamento das regiões de supressão espectral ao longo do eixo da frequência proporcional ao incremento de fase utilizado $\delta \varphi$ como descrito na seção 2.4.1.

De modo a eliminar as regiões de supressão, o método de múltiplas aquisições SSFPdx se baseia na soma de dois ou mais experimentos com diferentes incrementos de fase. A Fig. 5.9 esquematiza os perfis de excitação somados nos métodos baseados na combinação de aquisições SSFPdx, com 2, 4 e 8 blocos de incremento de fase.

Tais sequências - denominadas SSFPdx-n2, SSFPdx-n4 e SSFPdx-n8 - com múltiplas aquisições SSFPdx foram implementadas no software TopSpin da Bruker, para realizar as aquisições em sequência única. Escolhido o número de blocos (2, 4 ou 8 ) e definido o número de dummy scans $\left(L_{0}\right)$ e scans $\left(L_{1}\right)$ de cada bloco, a sequência executa as etapas:

1 - Aplica $L_{0}$ dummy scans até estabilização do estado estacionário;

2 - Aplica $L_{1}$ scans do sinal nesse primeiro estado estacionário;

3 - Imediatamente inicia próximo esquema de fase com $L_{0}$ dummy scans até estabilização; 

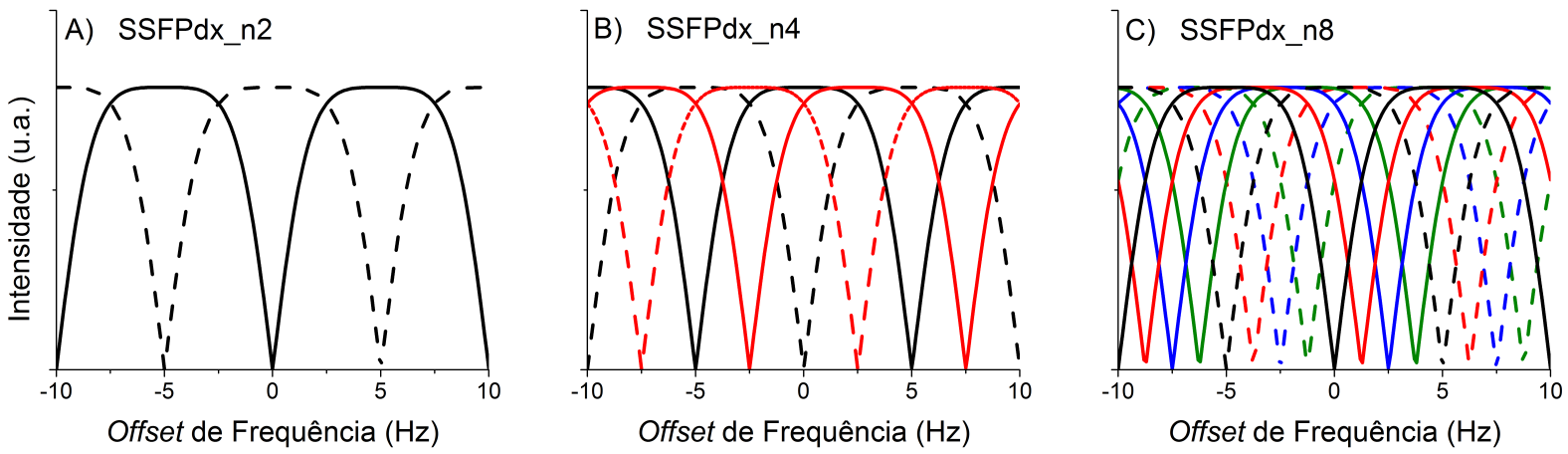

Figura 5.9- Múltiplas aquisições SSFPdx-n2, SSFPdx-n4 e SSFPdx-n8, utilizam-se respectivamente de 2, 4 e 8 blocos de aquisição SSFPdx, onde cada bloco possui um valor de incremento de fase $\delta \varphi$ de $0, \pi / 4, \pi / 2,3 \pi / 4, \pi, 5 \pi / 4,3 \pi / 2$ e $7 \pi / 4$.

Fonte: Elaborada pelo autor.

4 - Soma $L_{1}$ scans do sinal nesse segundo estado estacionário aos da etapa 2;

5 - Havendo mais blocos o procedimento se repete;

A sequência com 2 blocos, SSFPdx-n2, apresenta o primeiro bloco pulsos com incremento de fase 0 e o segundo com incremento $\pi$, correspondendo a soma dos perfis A) e E) da Fig. 5.8. A sequência com 4 blocos, SSFPdx-n4, apresenta blocos com incremento $0, \pi / 2, \pi$ e $3 \pi / 2$, correspondendo a soma dos perfis A), C), E) e H) da Fig. 5.8. E para a sequência com 8 blocos, SSFPdx-n8, incrementos de $0, \pi / 8, \pi / 4,3 \pi / 8, \pi, 5 \pi / 8,3 \pi / 4$ e $7 \pi / 8$, correspondendo a soma de todos os perfis da Fig. 5.8.

Vale ressaltar que a implementação de incrementos de fase $\delta \varphi$ não era uma tarefa trivial no antigo espectrômetro da Varian INOVA onde iniciamos o desenvolvimento do projeto, pois o sistema apresenta a pré-definição de apenas as fases $(x, y,-x,-y)$. Em espectrômetros mais modernos, como no AVANCE III da Bruker incrementos de fase foram facilmente introduzidos.

A Fig. 5.10 apresenta os perfis de excitação totais para essas sequências com 2, 4 e 8 blocos de aquisição para a amostra de água e para a mistura de água e acetona. Como é observado essas sequências removem as regiões de supressão, diminuindo as anomalias de fase e amplitude observadas nos espectros, sendo mais eficiente conforme maior número de blocos de fases que são utilizados.

A desvantagem de inserir muitos blocos de fases é a necessidade de esperar a estabilização de cada estado estacionário para realizar o próximo bloco de aquisição. Este tempo de estabilização é da ordem do maior $T_{1}$ da amostra, de modo que quando aumentamos demasiado 

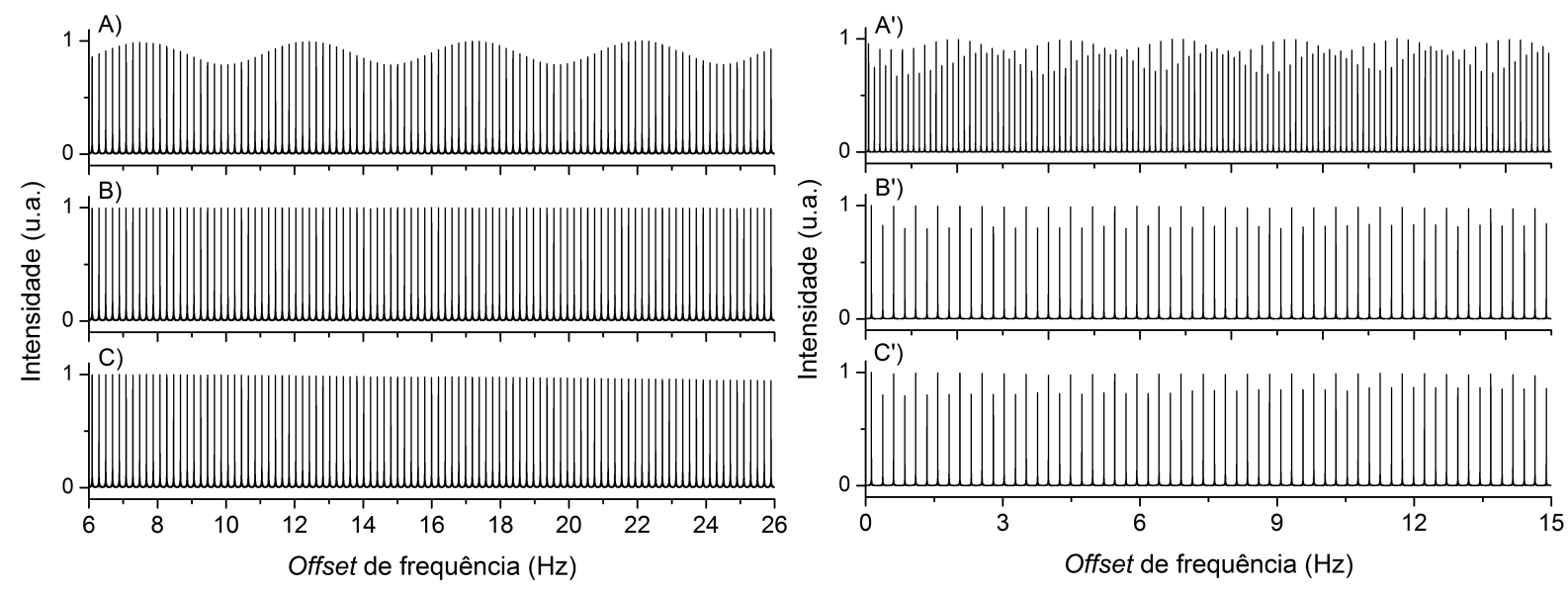

Figura 5.10- A direita perfil de excitação $\mathrm{H}_{2} \mathrm{O} / \mathrm{D}_{2} \mathrm{O}$ para sequências A) SSFPdx-n2, B) SSFPdx-n4 e C) SSFPdx-n8. A esquerda perfil de excitação água e acetona para sequências $A^{\prime}$ ) SSFPdx-n2, B') SSFPdx-n4 e C') SSFPdx-n8, todos adquiridos com $T_{p}=100 \mathrm{~ms}, \theta=90^{\circ}$ e 8 médias.

Fonte: Elaborada pelo autor.

o número de blocos de SSFPdx, elevamos o tempo total de aquisição do sinal.

Na próxima seção apresentamos a SSFPdxdt com incremento não-linear de fase, onde os blocos de dummy scans e múltiplas aquisições são desnecessários, simplificando e acelerando os experimentos.

\subsection{SSFPdxdt com incremento não-linear de fase}

Nas sequências SSFPdx com múltiplos blocos de aquisição apresentadas na seção anterior é sempre necessário adquirir múltiplos blocos SSFPdx e aplicar dummy scans na transição dos estados estacionários, o que eleva o tempo experimental total.

Na busca de uma sequência SSFP sem blocos de transição e que gere um perfil de excitação uniforme, verificamos que qualquer alternância de fase que utilize incremento de fase constante, sempre vai gerar regiões de máximo e mínimo de intensidade. Dessa forma, nossa proposta nesta seção é gerar uma sequência SSFP onde o perfil de excitação no estado estacionário seja deslocado vagarosamente ao longo do eixo de frequência.

Essa ideia foi realizada através de uma sequência SSFP com incremento não-linear de fase, aqui denominada SSFPdxdt. O termo $d x d t$ é estabelecido devido a analogia da técnica com o deslocamento do perfil de excitação ao longo do eixo da frequência. A fase do $n$-enésimo 
pulso é dada por

$$
\varphi(n)=\varphi_{0}+A \cdot(n-1)^{2}
$$

com $n=1,2,3, \ldots, N_{f}$, onde $N_{f}$ o último pulso da sequência. $A$ é uma constante que define um análogo a aceleração e $\varphi_{0}$ pode ser considerada nula no início. As sequências de pulsos SSFPdxdt são da forma

$$
\left[\theta_{x}-\theta_{x+1 A}-\theta_{x+4 A}-\theta_{x+9 A}-\theta_{x+16 A}-\theta_{x+25 A}-\ldots-\theta_{x+\left(N_{f}-1\right)^{2} A}\right]
$$

Uma série de simulações numéricas foram realizadas para examinar as propriedades da magnetização submetida a esse esquema de fase. A Fig. 5.11 apresenta simulações da magnetização para $A=\pi / 16, \pi / 32, \pi / 64, \pi / 128, \pi / 256, \pi / 512, \pi, 1024$ e $\pi / 2048$. Fig. 5.11 A) mostra que para $A$ menor que $\pi / 128$ a amplitude máxima do sinal cresce conforme menor o valor de $A$, e em B) após um valor pequeno o suficiente de $A$, no caso $\pi / 256$, a amplitude máxima dos sinais fica sempre em $M_{0} / 2$. Nas simulações foram utilizados $T_{1}=T_{2}=3 \mathrm{~s}, T_{2}^{*}$ $=160 \mathrm{~ms}, \theta=90^{\circ}$ e $T_{p}=100 \mathrm{~ms}$.
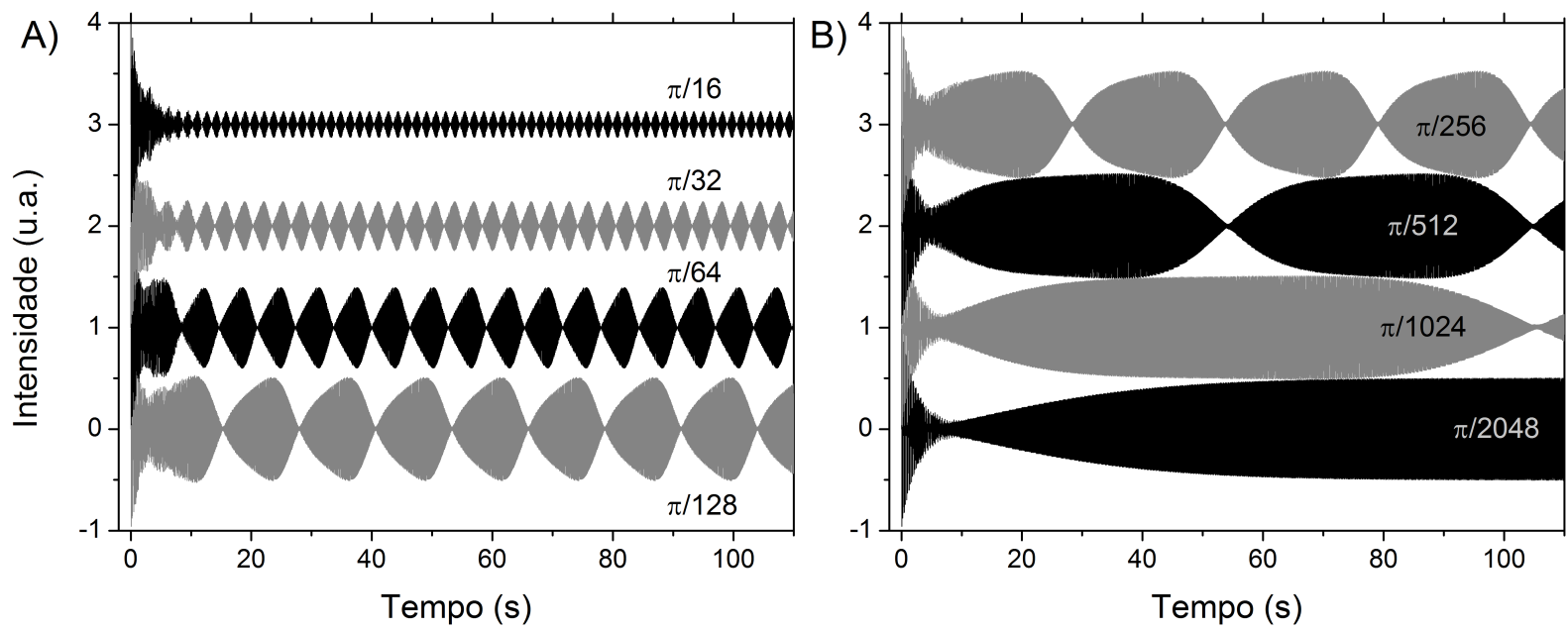

Figura 5.11- Sinais simulados da SSFPdxdt com incremento não-linear de fase. A magnetização no estado SSFPdxdt apresenta modulação temporal de acordo com o fator $A$ do incremento de fase utilizado onde apresentamos desde $A=\pi / 16$ até $\pi / 2048$. Em A) vemos que para $A$ menor que $\pi / 128$ a amplitude máxima do sinal cresce conforme menor o valor de $A$. Em B) vemos que após um valor pequeno o suficiente de $A$ a amplitude máxima dos sinais fica em $M_{0} / 2$.

Fonte: Elaborada pelo autor.

A modulação da amplitude da magnetização no tempo muda de acordo com o fator $A$ estabelecido. Temos que um período de oscilação da amplitude se completa a cada $\operatorname{div}(A)$ 
pulsos, onde $\operatorname{div}(A)$ é o divisor do fator $A$. Na escala de tempo o período é dado por

$$
T_{p e r}=T_{p} \cdot \operatorname{div}(A)
$$

Quando o fator $A$ é muito grande, a transição do perfil de excitação é muito rápida, de modo que a magnetização não tem tempo suficiente para se formar no estado estacionário, sofrendo supressão na amplitude. Esse é o motivo da amplitude máxima da Fig. 5.11 ser reduzida para valores de $A$ entre $\pi / 16$ e $\pi / 128$.

Na implementação prática, realizamos experimentos com os valores de $\mathrm{A}: \pi / 2 \pi / 4, \pi / 8$, $\pi / 16, \pi / 32, \pi / 64, \pi / 128$ e $\pi / 256$ no limite da memória disponível no computador, como discutiremos a seguir. O comportamento magnetização no eixo do tempo observado nas simulações da Fig. 5.11 concordam muito bem com o observado nos experimentos, Fig. 5.12.
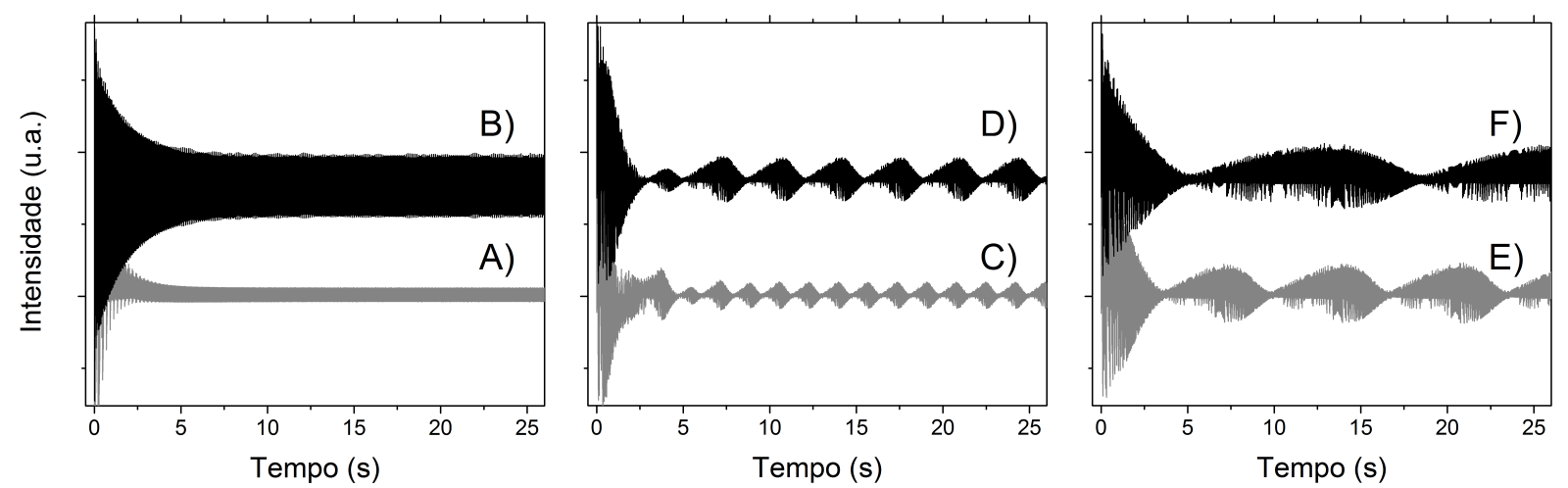

Figura 5.12- Sinais de $\mathrm{H}_{2} \mathrm{O} / \mathrm{D}_{2} \mathrm{O}$ apresentando o comportamento da magnetização quando submetida a sequência A) SSFP sem incremento de fase e na posição de mínima intensidade, B) SSFP sem incremento de fase e na posição de máxima intensidade, C) SSFPdxdt com $A=\pi / 32$, D) $A$ $=\pi / 64$, E) $\mathrm{A}=\pi / 128$ e F) $\mathrm{A}=\pi / 256$. Nas SSFPdxdt a amplitude no estado estacionário é modulada no tempo, alternando entre máximo e mínimo de intensidade ao longo do tempo, além disso a amplitude máxima do sinal é maior quanto menor valor de $\mathrm{A}$.

Fonte: Elaborada pelo autor.

Na Fig. 5.12 A) apresentamos a SSFP sem incremento de fase, na situação onde o pico da água está sofrendo supressão de sinal, estabelecendo um mínimo de intensidade no estado estacionário. Em B) temos a visualização de uma SSFP sem incremento de fase situando o pico da água na posição de máxima intensidade, como vemos após o tempo transiente, entre 0 e 5 segundos, a magnetização estabelece uma amplitude e nela permanece enquanto for continuada a aplicação dos pulsos de RF.

Os sinais da Fig. 5.12 C) até F) apresentam respectivamente a magnetização para as 
sequências SSFPdxdt com $\mathrm{A}=\pi / 32, \pi / 64, \pi / 128$ e $\pi / 256$. A diferença do método SSFPdxdt é que agora a amplitude da magnetização, mesmo no estado estacionário é modulada no tempo, apresentando uma transição periódica entre amplitude mínima e máxima de intensidade. Podemos notar também que quanto menor o valor de $\mathrm{A}(\pi / 256)$ máxima é a amplitude observada no estado estacionário.

A modulação do sinal no estado estacionário ao longo do tempo, sugere que o perfil de excitação das SSFPdxdt no eixo das frequências está em movimento, deslocando-se com velocidade dependente do valor $\mathrm{A}$ estabelecido. O significado disso é que a cada scan de aquisição o perfil de excitação é deslocado por um pequeno valor nos eixos da frequência, como explicado na Fig. 5.13. Um período dessa modulação está representado na figura pelas linhas tracejadas entre os tempos 13 e 27 segundos.
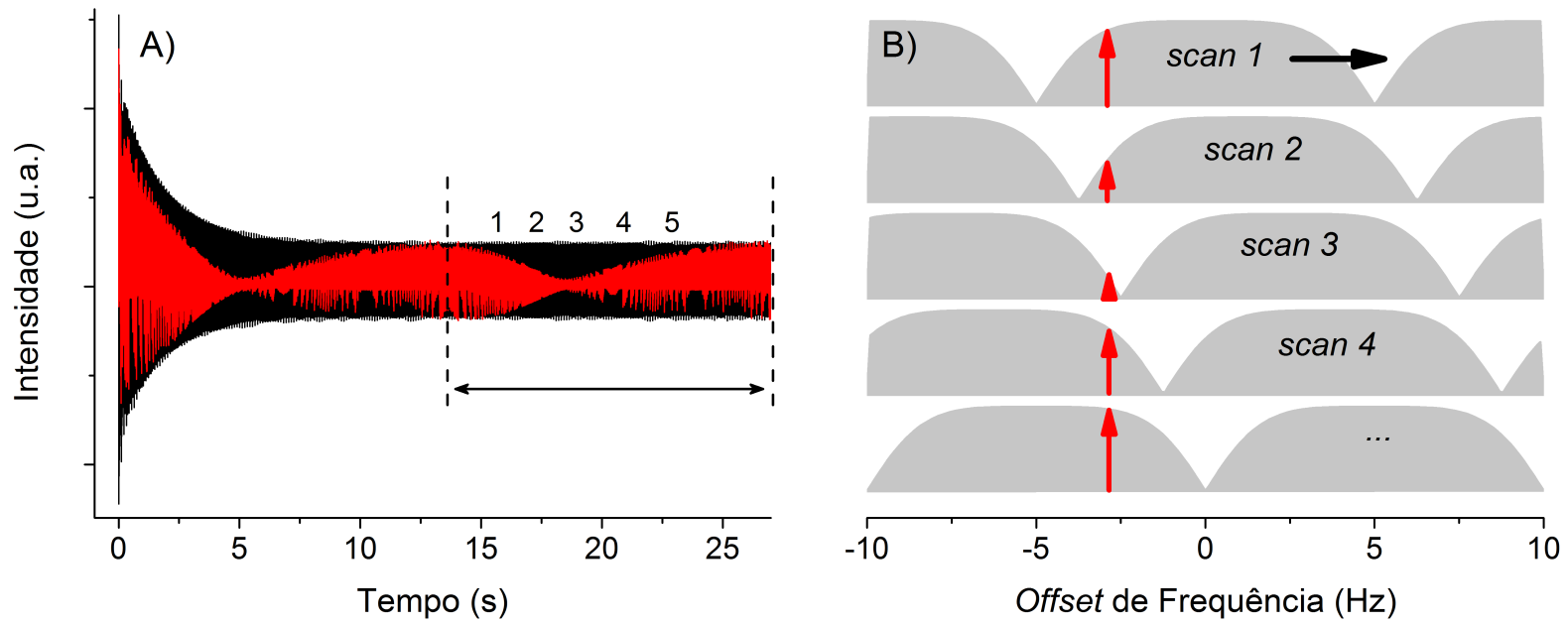

Figura 5.13- Em A) temos a comparação entre a SSFP sem incremento de fase (preto) em seu máximo de intensidade no estado estacionário, junto com o sinal SSFPdxdt usando $A=\pi / 256$ (vermeIho). Temos que a magnitude do sinal SSFPdxdt possui caráter periódico, correspondente ao deslocamento do perfil de excitação no eixo das frequências, como apresentado em B). A seta vermelha em B) representa um pico do espectro que em cada scan apresenta uma amplitude modulada correspondente as posições 1 à 5 de $A$ ).

Fonte: Elaborada pelo autor.

Para obter perfis de excitação uniforme nesse novo esquema de fase é necessário adquirir ao menos um período completo da oscilação da amplitude no eixo dos tempos. No espectrômetro AVANCE III da Bruker, implementamos esse incremento quadrático nas fases dos pulsos construindo listas de fases que completem um período, podendo repeti-las quantas 
vezes desejarmos. Por exemplo, para $A=\pi / 16$, temos as fases

$$
[0 ; 1 ; 4 ; 9 ; 16 ; 25 ; 36 ; 49 ; 64 ; 81 ; 100 ; 121 ; 144 ; 169 ; 196 ; 225]\left(\frac{\pi}{16}\right) .
$$

as fases do tipo $49 \pi / 16$ podem ser reduzidas para seu ângulo equivalente $4 \pi / 16$, e calculando a sequência acima para muitos pulsos, vemos que para $A=\pi / 16$ os termos abaixo se repetem indefinidamente

$$
[0 ; 1 ; 4 ; 9 ; 16 ; 25 ; 4 ; 17 ; 0 ; 17 ; 4 ; 25 ; 16 ; 9 ; 4 ; 1]
$$

sempre com número de elementos igual ao termo do denominador de $A$. Esse ciclo de repetição encontrado representa o ciclo completo do perfil de excitação no eixo da frequências, retornando o ponto de mínimo de amplitude para a posição inicial.

Dessa forma, a implementação da sequência de pulsos SSFPdxdt envolveu os parâmetros $L_{0}$ para aplicar dummy scans no transiente inicial da magnetização, e após esse transiente estabelece o estado estacionário com perfil de excitação modulado no tempo. O número de scans realizados na sequência é definido por $L_{1}$, onde ambos devem ser um múltiplo do divisor do fator $\mathrm{A}, L_{0,1}=n \operatorname{div}(A)$. Garantindo essas condição para $L_{0}$ e $L_{1}$ um perfil de excitação uniforme será obtido. Para nosso menor fator utilizado $A=\pi / 256$ o número de scans mínimo é 256 .

A Tabela 5.1 apresenta a lista de fase utilizada utilizada para $A$ igual a $\pi / 2, \pi / 4, \pi / 8$, $\pi / 16, \pi / 32, \pi / 64, \pi / 128$ e $\pi / 256$. A lista de fases para $\pi / 512$ ou menores não é apresentada, pois ao implementarmos no espectrômetro AVANCE III não houve memória computacional para executar tais sequências. De qualquer modo a sequência com $A=\pi / 256$ apresenta amplitude máxima igual a máxima da SSFP convencional, que representa o limite de amplitude, como sugerem as simulações apresentadas na Fig. 5.11.

A Fig. 5.14 compara o perfil de excitação da amostra de água com acetona, para as sequências SSFPdxdt com A igual a A) $\pi / 256$, B) $\pi / 128$, C) $\pi / 64$, D) $\pi / 32$, E) $\pi / 16$ e com a SSFP sem incremento de fase em F). Todos espectros adquiridos com 256 médias e $T_{p}=200 \mathrm{~ms}$. Resultados mostram os perfis com a SSFPdxdt são uniformes e a amplitude dos mesmos é maior quanto menor o fator A, como mostra a Fig 5.12. 
Tabela 5.1- Esquema de fases da SSFPdxdt para A igual a $\pi / 2, \pi / 4, \pi / 8, \pi / 16, \pi / 32, \pi / 64, \pi / 128$ e $\pi / 256$.

\begin{tabular}{|c|c|}
\hline Incr. & Fases \\
\hline$\pi / 2$ & 01 \\
\hline$\pi / 4$ & 0141 \\
\hline$\pi / 8$ & 01490941 \\
\hline$\pi / 16$ & 0149162541701742516941 \\
\hline$\pi / 32$ & 0149162536490173657164143303344116573617049362516941 \\
\hline$\pi / 64$ & $\begin{array}{l}01491625364964811001211641689703368105165710017641133689 \\
1673465065473168936113641710057161056833097684116121100 \\
816449362516941\end{array}$ \\
\hline$\pi / 128$ & 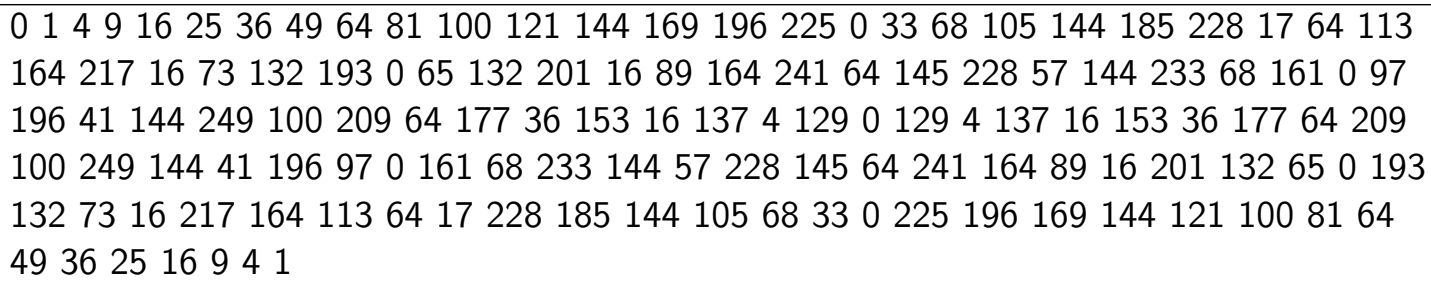 \\
\hline$\pi / 256$ & 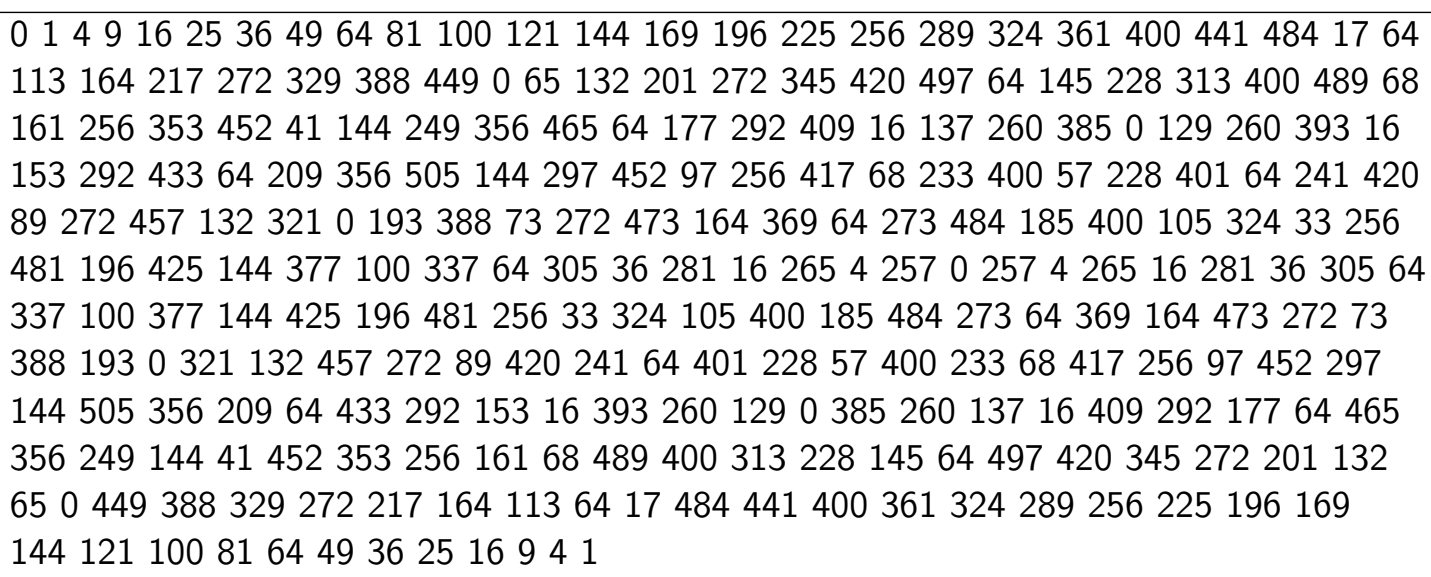 \\
\hline
\end{tabular}

Fonte: Elaborada pelo autor.

Como esperado a amplitude da SSFPdxdt com $\mathrm{A}=\pi / 256$ é menor e representa a média do sinal da SSFP sem incremento de fase em F). Apesar de perder amplitude, a geração de um perfil de excitação uniforme elimina anomalias de fase e amplide dos espectros, como apresentaremos na seção seguinte.

Resultados com A inferior a $\pi / 256$ não foram obtidos até a finalização deste trabalho devido a limitação de memória computacional, porém estratégias de programação para superar essa limitação estão em desenvolvimento.

\subsection{Núcleos com baixa sensibilidade}

A principal vantagem das sequências SSFP na aquisição de espectros em alta resolução é o grande ganho no tempo total da realização dos experimentos. Desse modo a técnica se torna 


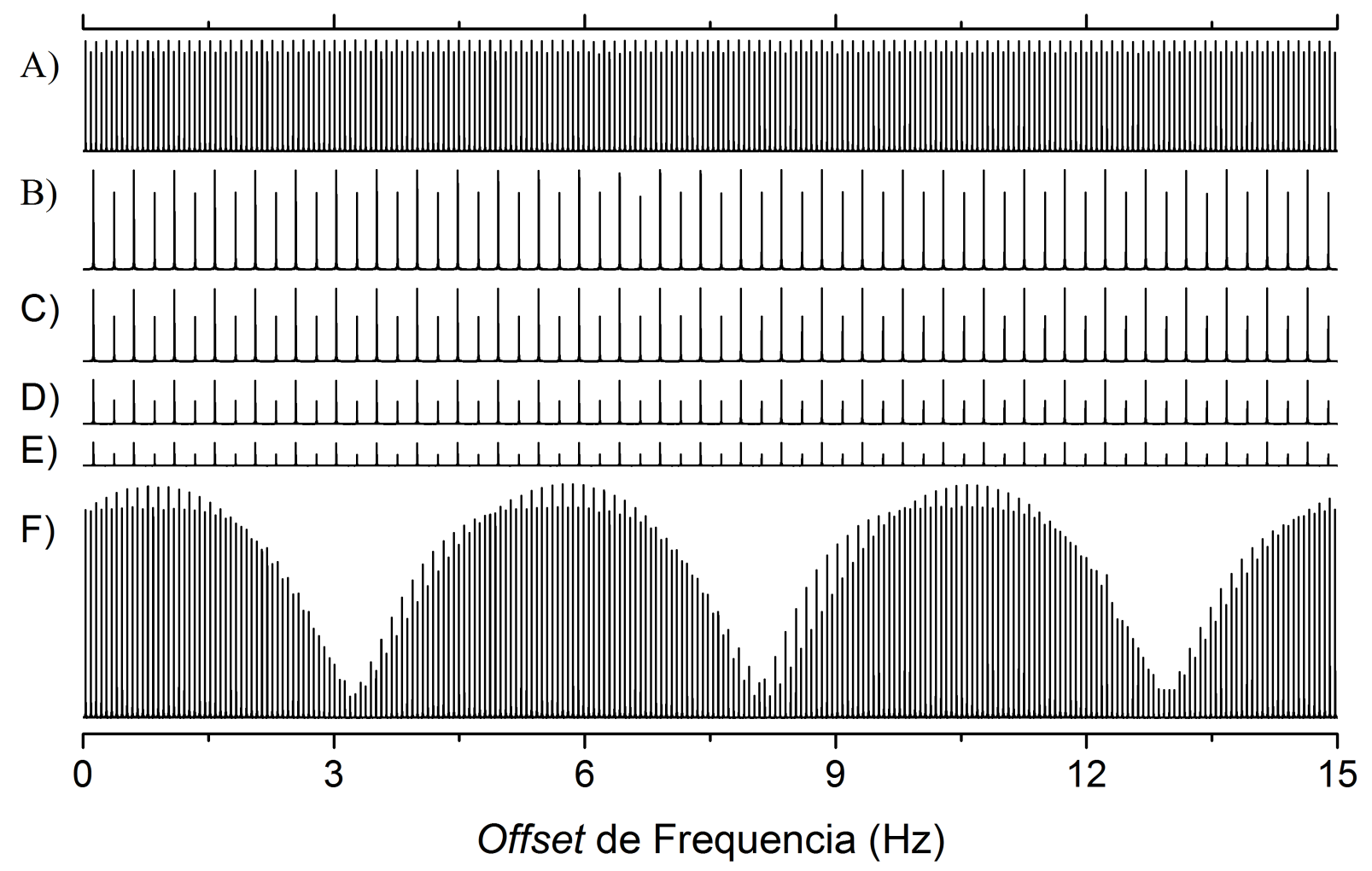

Figura 5.14- Comparação entre perfil de excitação das sequências SSFPdxdt com A sendo A) $\pi / 256$, B) $\pi / 128$, C) $\pi / 64$, D) $\pi / 32$, E) $\pi / 16$ e com a SSFP sem incremento de fase em F).

Fonte: Elaborada pelo autor.

interessante na obtenção de espectros de núcleos com baixa sensibilidade, tais como ${ }^{13} \mathrm{C},{ }^{15} \mathrm{~N}$ e ${ }^{31} P$, onde experimentos convencionais podem demorar horas ou até dias.

Nesta seção analisamos espectros adquiridos com técnicas convencionais, tais como zg e zgpg30 do TopSpin da Bruker, mostrando que espectros que levariam horas de aquisição podem ser obtidos com a mesma relação s/r em questão de minutos, as vantagens e desvantagem das sequências SSFPdx e SSFPdxdt serão discutidas.

\subsubsection{Carbono-13}

A Fig. 5.15 apresenta espectros de ${ }^{13} \mathrm{C}$ de $3,0 \mathrm{mg}$ de sacarose dissolvido em $500 \mu \mathrm{l}$ de $D_{2} O$, adquiridos com a sequência de pulso convencional (zgpg30 - Bruker) e com as sequências SSFPdx com 2, 4 e 8 blocos. A Tabela 5.2 apresenta os parâmetros de aquisição e os tempos necessários para cada aquisição.

O espectro de ${ }^{13} C$ padrão da Sacarose foi adquiridos com a sequência zgpg30 com 8192 médias, totalizando $6 \mathrm{~h} 46$ minutos de experimento. De modo a compararmos com as sequên- 
Tabela 5.2- Parâmetros utilizados nas sequências de pulso padrão (zgpg30), SSFP, SSFPdx-n2, SSFPdx-n4 e SSFPdx-n8.

\begin{tabular}{c||c|c|c|c|c}
\hline Seq. Pulso & zgpg30 & SSFP & SSFPdx-n2 & SSFPdx-n4 & SSFPdx-n8 \\
\hline \hline Tempo aquisição $($ at) & $1,3 \mathrm{~s}$ & $99,7 \mathrm{~ms}$ & $99,7 \mathrm{~ms}$ & $99,7 \mathrm{~ms}$ & $99,7 \mathrm{~ms}$ \\
\hline Tempo de reciclo $\left(d_{1}\right)$ & $1,0 \mathrm{~s}$ & $0,3 \mathrm{~ms}$ & $0,3 \mathrm{~ms}$ & $0,3 \mathrm{~ms}$ & $0,3 \mathrm{~ms}$ \\
\hline Ângulo pulso $(\theta)$ & $30^{\circ}$ & $90^{\circ}$ & $90^{\circ}$ & $90^{\circ}$ & $90^{\circ}$ \\
\hline Tempo total experimento & $6 \mathrm{~h} 46 \mathrm{~m}$ & $14 \mathrm{~m}$ & $14 \mathrm{~m}$ & $14 \mathrm{~m}$ & $14 \mathrm{~m}$ \\
\hline Razão s/r & 50 & 40 & 35 & 36 & 31 \\
\hline
\end{tabular}

Fonte: Elaborada pelo autor.

cias SSFPdx realizamos inicialmente as aquisições fixando o números de médias, assim os espectros SSFPdx apresentam 8192 médias em um tempo total de aquisição de 14 minutos. A comparação entre os espectros obtidos é apresentada na Fig. 5.15. Em A) o espectro adquirido com a sequência padrão (zgpg30) com 6h46m de aquisição. B) apresenta a SSFP sem incremento de fase adquirida em 14 minutos, com a presença de forte anomalias de fase e amplitude. Entre C) e E) apresentamos as sequências SSFPdx com 2, 4 e 8 blocos, onde se observa a melhora considerável na relação de amplitude dos picos, estabelecendo um perfil mais uniforme e reduzindo as anomalias de fase, com tempo total de aquisição de 14 minutos.

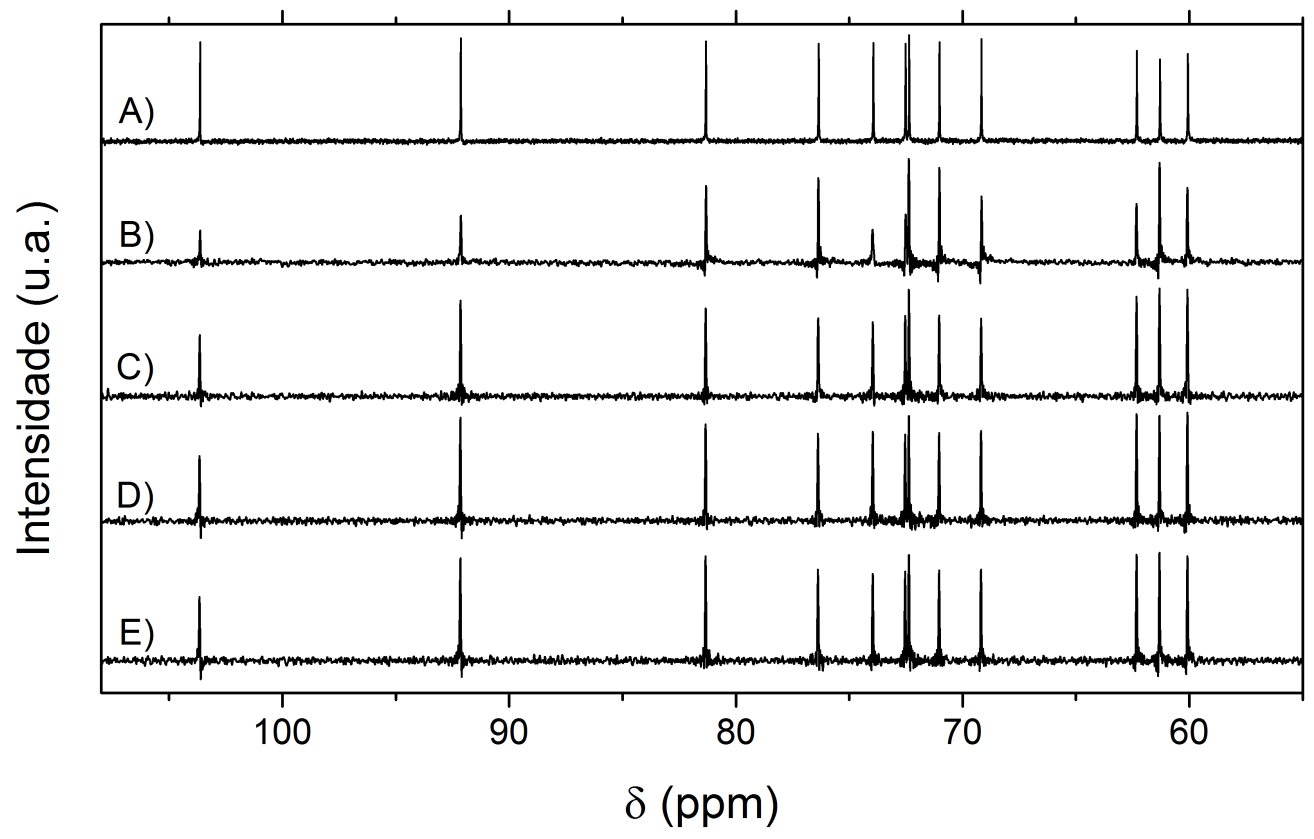

Figura 5.15- Comparação entre os espectros de ${ }^{13} C$ Sacarose adquiridos com as sequências A) padrão (zgpg30), B) SSFP, C) SSFPdx-n2, D) SSFPdx-n4 e E) SSFPdx-n8, todos com 8192 médias Fonte: Elaborada pelo autor.

A redução do tempo total de experimento de $6 \mathrm{~h} 46 \mathrm{~m}$ para apenas 14 minutos não vem sem custos. Na SSFP sem alternância de fases a presença das fortes anomalias de fase e amplitude 
são claras, além de muitas vezes a total supressão de picos espectrais com presença dos artefatos do truncamento e menor resolução. A SSFPdx consegue melhorar consideravelmente as anomalias espectrais observadas, porém a razão $\mathrm{s} / \mathrm{r}$ obtida para o mesmo número de médias é menor, uma vez que a soma dos sinais muda em cada bloco de aquisição, resultando em um valor médio da amplitude dos blocos. Evidentemente a razão s/r na SSFPdx pode ser melhorada com o aumento no número de scans total, mantendo ainda um tempo total de aquisição do sinal reduzido.

Vamos agora realizar uma comparação semelhante a anterior comparando a SSFPdxdt com aquisição padrão. Dessa vez utilizamos uma concentração maior de sacarose, com a solução de $105 \mathrm{mg}$ em $500 \mu \mathrm{l}$ de $D_{2} O$. A Fig. 5.16 apresenta espectros de ${ }^{13} \mathrm{C}$ dessa solução adquiridos em A) com padrão (zgpg30), B) com a SSFP sem alternância de fase e adquiridos com SSFPdxdt com incremento de fase em C) $\pi / 256$, D) $\pi / 64$ e E) $\pi / 16$, com 256 médias e $T_{p}=200 \mathrm{~ms}$.
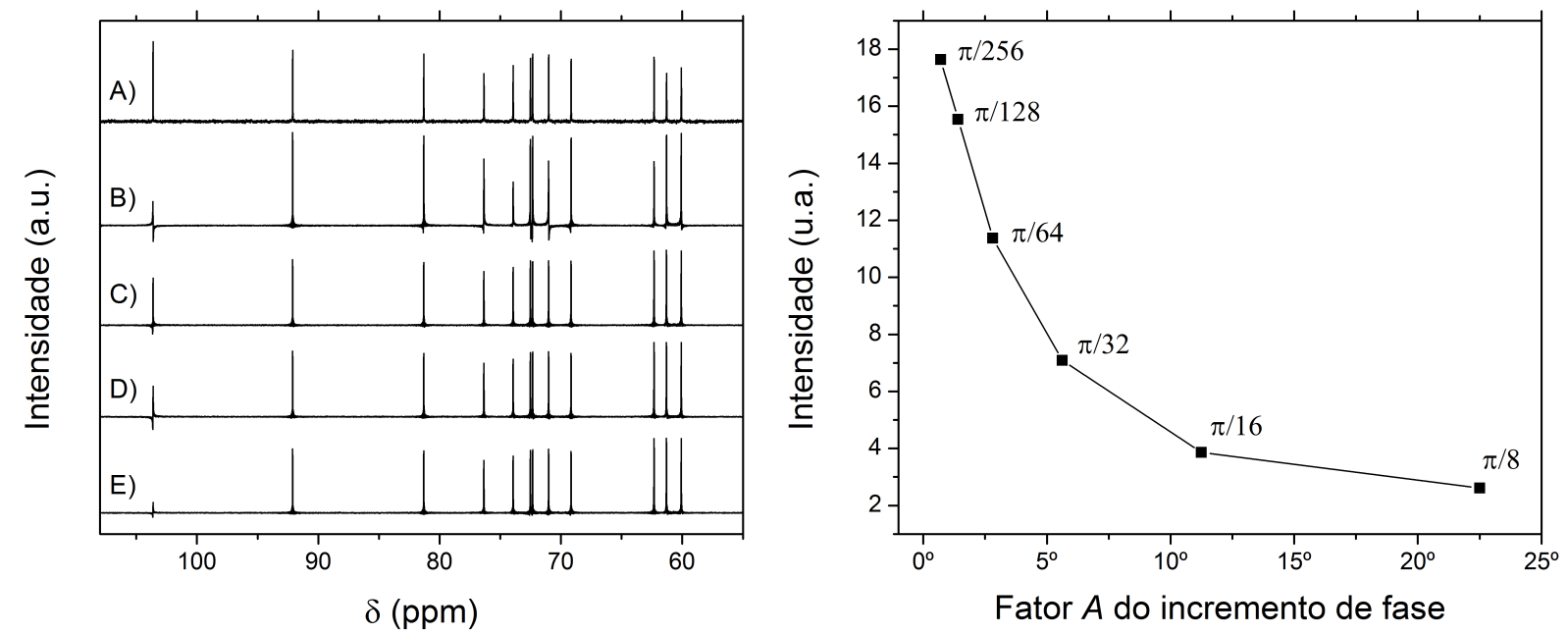

Figura 5.16- A esquerda comparação dos espectros de ${ }^{13} \mathrm{C}$ da sacarose adquiridos com as sequências $\mathrm{A}$ ) padrão (zgpg30), B) SSFP sem alternância de fase e adquiridos com SSFPdxdt com incremento de fase em C) $p i / 256$, D) $\pi / 64$ e E) $\pi / 16$, com 256 médias, $T_{p}=200$ ms. A direita intensidade do pico em 104 ppm em função do incremento de fase na SSFPdxdt.

Fonte: Elaborada pelo autor.

O interessante da Fig. 5.16 é analisar o comportamento das sequências SSFPdxdt com os diferentes incrementos de fase. Nota-se que o primeiro pico a esquerda, em 104 ppm, tem maior intensidade conforme menor o incremento de fase utilizado, isso está de acordo com o observado nos perfis de excitação da Fig. 5.14. Essa mudança de amplitude em relação aos demais picos do espectro aparenta ser devido aos tempos de relaxação $T_{1}$ e $T_{2}$ deles. 
Na Tabela 5.3 apresentamos os valores dos tempos de relaxação dos picos obtidos com as sequências de pulsos cpmg (cpmg) e inversão recuperação (t1irpg).

Tabela 5.3- Tempos de relaxação $T_{1}$ e $T_{2}$ para cada pico do espectro de ${ }^{13} C$ da sacarose, obtidos pelas técnicas cpmg e t1irpg

\begin{tabular}{c||c|c|c|c|c|c|c|c|c}
\hline$\delta(\mathrm{ppm})$ & 104 & 92 & 81 & 76 & 74 & 73 & 71 & 69 & 62 \\
\hline \hline$T_{1}(\mathrm{~s})$ & 6,0 & 0,44 & 0,44 & 0,45 & 0,45 & 0,46 & 0,46 & 0,47 & 0,30 \\
\hline$T_{2}(\mathrm{~s})$ & 5,6 & 0,17 & 0,26 & 0,32 & 0,26 & 0,45 & 0,33 & 0,38 & 0,30 \\
\hline$T_{1} / T_{2}$ & 1,1 & 2,6 & 1,7 & 1,4 & 1,7 & 1,0 & 1,4 & 1,2 & 1,0 \\
\hline
\end{tabular}

Fonte: Elaborada pelo autor.

Os resultados mostram que os tempos de relaxação do pico em 104 ppm é cerca de dez vezes superior aos demais picos do espectro, possuindo portanto um tempo para estabilização do estado estacionário mais elevado. Isso evidência que para picos com tempos de relaxação elevados a SSFPdxdt apresenta maior amplitude quanto menor for o incremento de fase utilizado. Uma vez que o incremento de fase defini a velocidade com que o perfil de excitação se desloca ao longo do eixo de frequências do espectro, quanto mais rápida for essa transição, mais saturado fica o sinal, não alcançando sua amplitude máxima durante o ciclo na SSFPdxdt, resultando em picos com baixa intensidade para tempos de relaxação mais longos.

Para analisar o efeito de sinais com tempos de relaxação muito longos na SSFPdxdt, selecionamos uma solução de etil benzeno $\left(C_{8} H_{10}\right)$ em clorofórmio deuterado $\left(C D C l_{3}\right)$. Iniciamos medindo os tempos de relaxação dos picos espectrais do ${ }^{13} C$ com as sequências cpmg e t1irpg, com os resultados subscrito nos picos do espectro da Fig. 5.17 A), com unidade de segundos.

O espectro na Fig. 5.17 A) foi adquirido com a sequência de pulsos zgig (inverse gated decoupling) para obtermos um espectro quantitativo com correta relação de amplitude entre os picos sem presença do efeito NOE (Nuclear Overhauser effect). Para isso foi necessário também utilizarmos o tempo de reciclo igual a $5 T_{1}$ da componente mais longa do espectro. A componente com maior valor de tempo de relaxação pertence ao clorofórmio, tripleto em 77 ppm, com $T_{1}=52 \mathrm{~s}$, de modo que a aquisição de 8 médias desse sinal levou cerca de 30 minutos.

Na Fig. 5.17 A) vemos a correta relação de amplitude entre os picos do espectro, mostrando que a proporção de clorofórmio é superior aos demais picos. Em B) e C) apresentamos espectros adquiridos com a SSFPdxdt com incremento de fase $\pi / 256$ e a SSFPdx-n8 respec- 

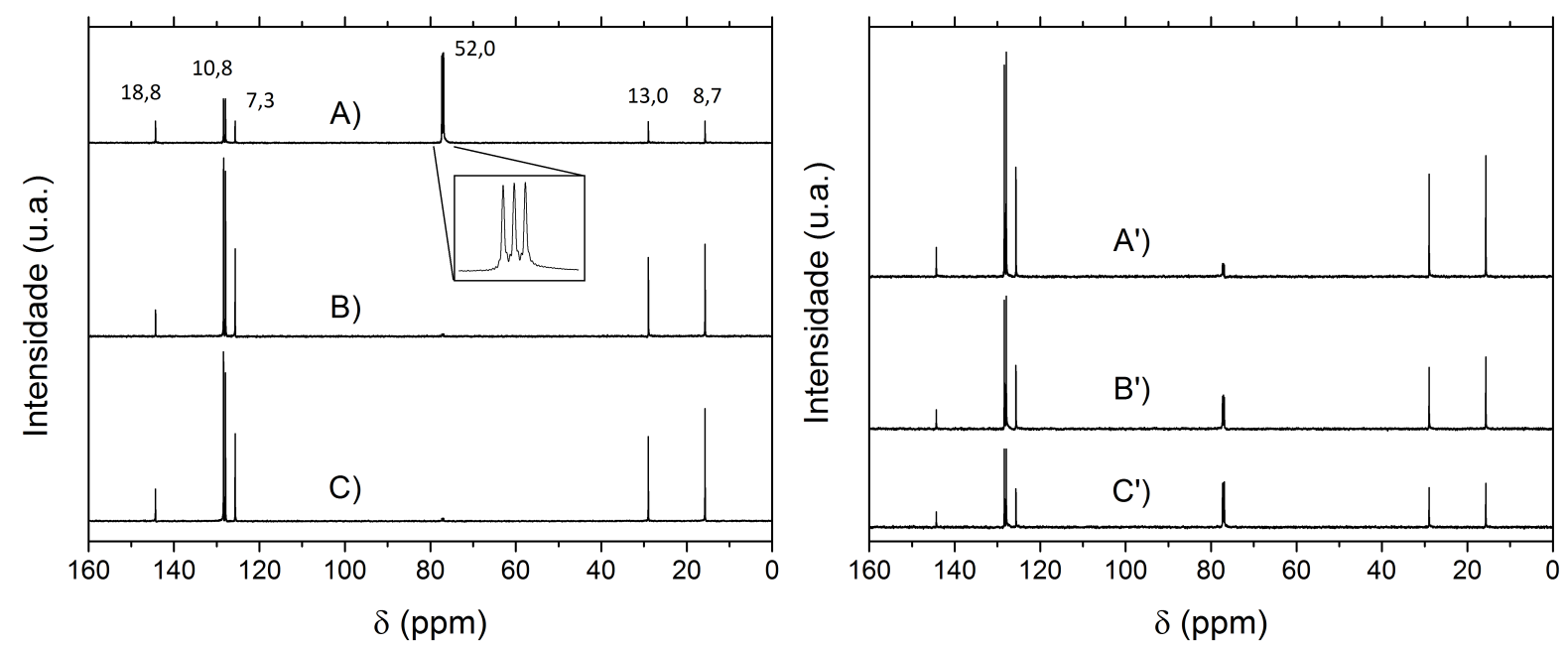

Figura 5.17- Espectros de ${ }^{13} \mathrm{C}$ do etil bezeno em clorofórmio com aquisição pela sequência A) zgig, B) SSFPdxdt $\pi / 256$, C) SSFPdxdt-n8. A direita espectros adquiridos com a SSFPdxdt $\pi / 256 \mathrm{com}$ pulsos de refocalização de $\left.\left.A^{\prime}\right) \theta=30^{\circ}, B^{\prime}\right) 10^{\circ}$ e $\left.C^{\prime}\right) 5^{\circ}$.

Fonte: Elaborada pelo autor.

tivamente. Em ambos os casos houve a quase total supressão dos picos do clorofórmio em 77 ppm. Apesar da transição lenta entre os estado estacionários os picos do clorofórmio foram suprimidos. Nesse caso, isso ocorre devido a razão $T_{1} / T_{2}$ do pico ser elevada. Quando $T_{1} / T_{2}$ é alto, pela equação 2.2.18 vimos que a amplitude da magnetização no estado estacionário torna-se muito pequena para pulsos de $90^{\circ}$. Nessas situações, como descrito na Fig. 2.5, pulso com ângulos de refocalização menor apresentam picos com maior amplitude.

O efeito da aplicação de pulsos com menor ângulo de refocalização na SSFPdxdt com incremento de fase $\pi / 256$ pode ser observado Fig. 5.17 com A') $\left.30^{\circ}, B^{\prime}\right) 10^{\circ}$ e $\left.C^{\prime}\right) 5^{\circ}$. Temos que a amplitude do clorofórmio cresce conforme menor o ângulo e um ângulo ótimo para a amplitude pode ser calculado conhecendo o valor da razão $T_{1} / T_{2}$. Deve-se levar em conta também o fato de que os picos com outros valores de razão $T_{1} / T_{2}$ ao longo do espectro iram sofrer alterações na amplitude com a mudança do ângulo dos pulsos. Isso pode ser observado com o decréscimo dos picos em torno de 130 e 20 ppm.

Mais resultados de espectros de ${ }^{13} \mathrm{C}$ adquiridos com a técnicas SSFPdx e SSFPdxdt são apresentadas na seção 6.1 com discussão conjunta da utilização do processamento com o KBDM. 


\subsubsection{Nitrogênio-15}

Núcleos de ${ }^{15} \mathrm{~N}$ apresentam sensibilidade 16 vezes menor que de ${ }^{13} C$, exigindo experimentos com duração que pode demorar dias tornando essas análise inviável em determinadas aplicações. Algumas estratégias para aquisição rápida de espectros de ${ }^{15} \mathrm{~N}$ são os custosos processos de enriquecimento de compostos e técnicas de transferência de polarização. A proposta da aplicação da SSFPdxdt na detecção de espectros de ${ }^{15} \mathrm{~N}$ vem como uma alternativa de fácil implementação e baixo custo para avaliações qualitativas rápidas de amostra de baixa sensibilidade.

A Fig. 5.18 apresenta espectros de ${ }^{15} \mathrm{~N}$ de uma solução de $200 \mu \mathrm{l}$ de anilina $\left(\mathrm{C}_{6} \mathrm{H}_{7} \mathrm{~N}\right)$, $200 \mu \mathrm{l}$ de trietilamina $\left(C_{6} H_{15} N\right)$ em $200 \mu$ l acetronitrila deuterada $\left(C D_{3} C N\right)$. Em A) temos o espectro padrão (zg30) com 2048 médias em um tempo total de aquisição de 3 horas e 30 minutos. B) apresenta o espectro adquirido com a SSFPdxdt $\pi / 256$ com tempo de aquisição de 30 minutos e $T_{p}=200 \mathrm{~ms}, 8192$ médias. Razão $\mathrm{s} / \mathrm{r}$ similar foi obtidas em ambos os casos, com um ganho de tempo na SSFPdxdt de 7 vezes.
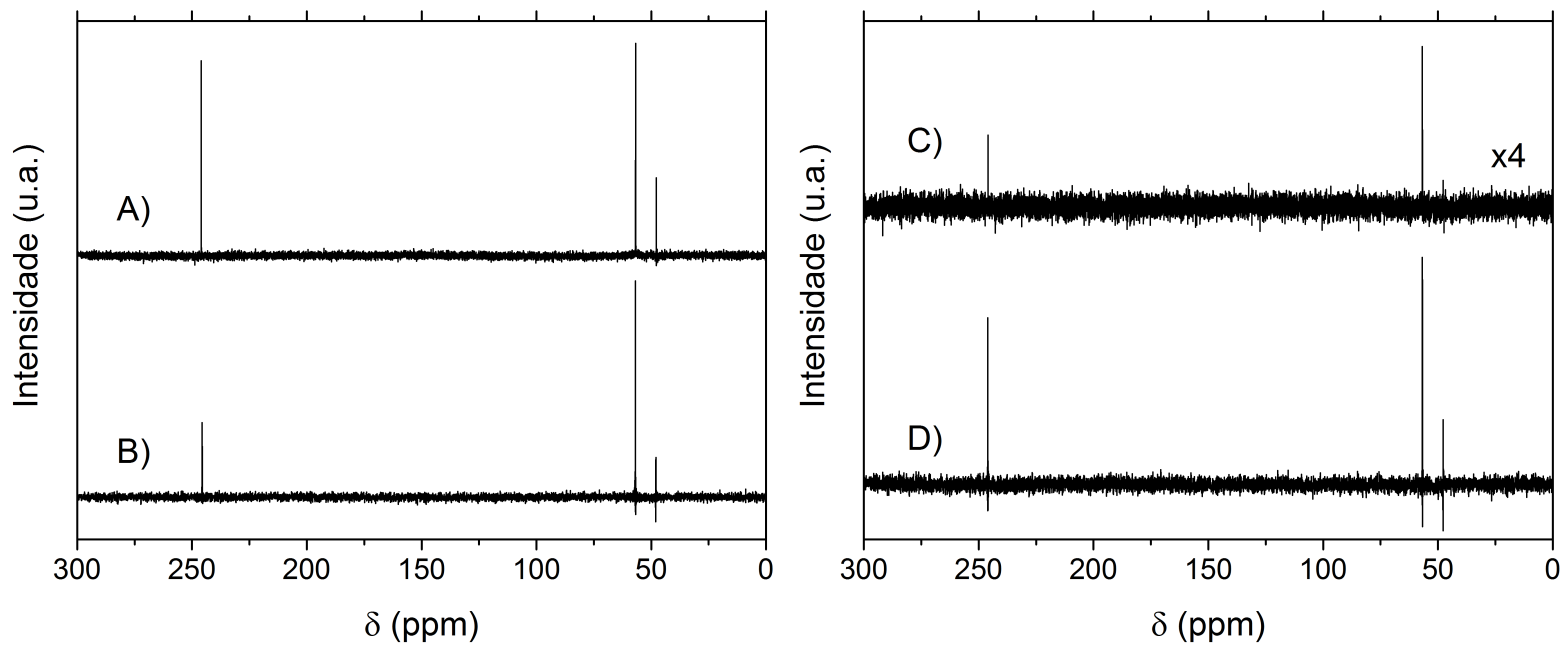

Figura 5.18- Espectros de ${ }^{15} \mathrm{~N}$ de anilina e trietilamina para as sequências A) padrão (zg30) com $3 \mathrm{~h}$ e 30 minutos de aquisição, B) SSFPdxdt $\pi / 256$ com 30 minutos de aquisição, C) padrão com 15 minutos aquisição e D) SSFPdxdt $\pi / 256$ com 15 minutos de aquisição. O espectro C) foi ampliado 4 vezes para melhor visualização.

Fonte: Elaborada pelo autor.

Na Fig. 5.18 C) e D) comparamos respectivamente a aquisição pela sequência padrão (zg30) e SSFPdxdt fixando tempo total de cada experimento em 15 minutos. Resultado mostra que pela sequência padrão, o espectro apresenta razão $s / r$ muito inferior não resolvendo todos os picos. 
Outro exemplo de aquisição ${ }^{15} \mathrm{~N}$ é apresentado na Fig. 5.19, com espectros de Formamida $\left(\mathrm{CH}_{3} \mathrm{NO}\right)$ com dimetilsulfóxido $D M S O$. Em A) temos o espectro adquirido com a sequência de pulsos padrão (zg30). Os demais espectros foram adquiridos com a sequência de pulso B) SSFP sem alternância de fase C) SSFPdx-n8 e de D) à H) com a SSFPdxdt $\pi / 256$ com processamento por Transformada de Fourier e KBDM.

O espectro padrão em A) foi adquirido com $t_{a q}=2 \mathrm{~s}$ e 256 médias. Em B) o espectro foi adquirido com a SSFP com $T_{p}=100 \mathrm{~ms}$, onde vemos que o pico em 112,5 ppm foi suprimido, além de termos grande perda de resolução com sobreposição do dubleto em 113 ppm. Em C) e D) temos respectivamente o espectro adquirido com a SSFPdx-n8 e a SSFPdxdt $\pi / 256$, ambos com $T_{p}=100 \mathrm{~ms}$, que apesar de evitarem a supressão do pico anterior, não apresentam resolução na definição dos dubletos devido o forte truncamento do sinal.

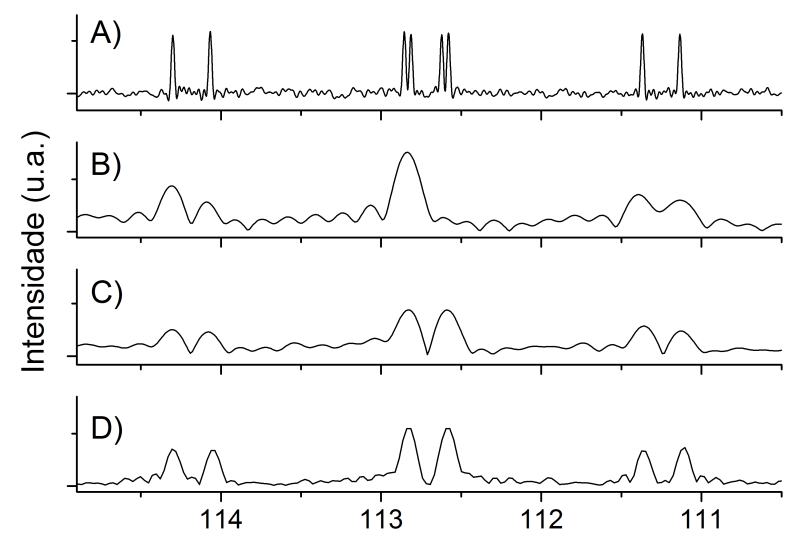

(ठ) ppm
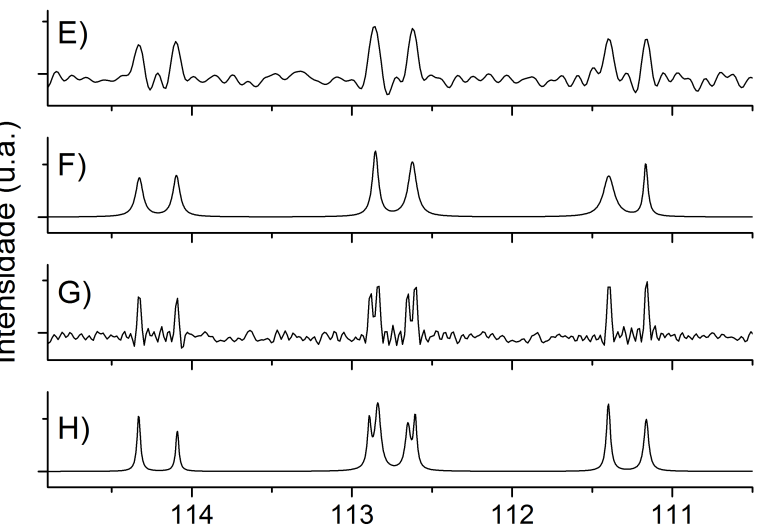

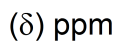

Figura 5.19- Espectros de ${ }^{15} \mathrm{~N}$ da Formamida $\left(\mathrm{CH}_{3} \mathrm{NO}\right)$ para as sequências: A) Padrão (zg), B) SSFP, C) SSFPdx-n8 e D) SSFPdxdt com $\pi / 256$ e $T_{p}=100 \mathrm{~ms}$. E) e F) apresenta o sinal SSFPdxdt adquirido com $T_{p}=200 \mathrm{~ms}$, processado respectivamente com a TF e KBDM. G) e H) sinal SSFPdxdt adquirido com $T_{p}=400 \mathrm{~ms}$, processado respectivamente com a TF e KBDM.

Fonte: Elaborada pelo autor.

Os espectros da Fig.5.19 E) à $\mathrm{H}$ ) foram adquiridos com a SSFPdxdt $\pi / 256$ com $T_{p}=$ $200 \mathrm{~ms}$ e $400 \mathrm{~ms}$, processados com a Transformada de Fourier e pelo KBDM. Evidentemente as técnicas SSFPdx e SSFPdxdt superaram a supressão espectral, porém um limitante dos métodos é a baixa resolução espectral devido o forte truncamento do sinais. Esses sinais truncados devem ser tratados com funções de apodização especiais ou por outros métodos especiais de processamento, como o FDM e KBDM. Na seção seguinte 5.5 discutimos a questão da resolução espectral obtida. 


\subsection{Resolução, tempo e razão sinal/ruído}

A relação entre os fatores: resolução, tempo experimental e razão sinal/ruído podem ser imaginadas na analogia do triângulo $(\triangle)$ onde cada uma representa um vértice. Ao escolher dois fatores ótimos, o 'custo' cairá sobre a terceira, que apresentará problemas. Por exemplo, para ter boa razão s/r com boa resolução, demandará tempo experimental. Se escolhermos ter boa resolução num pequeno tempo experimental, teremos então baixa razão s/r. A terceira possibilidade é buscar boa razão $\mathrm{s} / \mathrm{r}$ em curto tempo experimental, resultando em baixa resolução.

A técnica SSFP se encaixa no último caso, onde na busca por um curto tempo experimental com boa razão $s / r$, resultamos em sinais FID truncados e espectros com baixa resolução. Resolução é a capacidade de distinguir sinais com frequências muito próximas. A resolução pode ser limitada intrinsecamente pela sistema físico e/ou aparato dos detectores, mas também é frequentemente afetada pelos métodos numéricos utilizados no processamento dos dados. $\mathrm{Na}$ Transformada de Fourier discreta a resolução é limitada por $\delta \omega \approx \frac{1}{N \tau}$, onde $\mathrm{N}$ é o número de pontos e $\tau$ o espaçamento entre eles.

O tempo de aquisição dos sinais SSFP são da ordem de $m s$, de forma que devido ao longo tempo de relaxação dos núcleos - da ordem de segundos - os sinais adquiridos tipicamente apresentam pouco decaimento. Esse FID truncado é equivalente a multiplicarmos o sinal por uma função retangular, que na Transformada de Fourier leva a introdução de artefatos sinc $\left(\frac{\operatorname{sen}(x)}{x}\right)$ ao redor dos picos, Fig. 5.19. Esses artefatos sinc (batimentos) são gerados devido a limitação de resolução da DFT, e podem ser evitados aumentando os tempos de aquisição dos sinais ou então multiplicando o FID por uma função de apodização (LB). No caso da SSFP, em algumas situações a apodização consegue eliminar os batimentos, porém em espectros mais complexos, um elevado número de picos introduz sérias anomalias ao logo de todo espectro.

A resolução do espectro costuma ser melhorada utilizando o procedimento de zero-filling, que não resolvem o problema real, uma vez que isso apenas introduz resolução digital, com informação que já estava no sinal. O ganho de resolução obtido em outros métodos de processamento geralmente vêm da introdução de informação prévia a análise.

Nos métodos paramétricos tipo FDM e KBDM, é assumido que o sinal é composto de uma 
soma de exponencias oscilatórias amortecidas. Em princípio, o KBDM necessita de apenas de 2 pontos complexos por pico lorentziano para resolver um espectro, onde no caso da Fig. 5.19 A) com 8 picos, no mínimo deveríamos adquirir 16 pontos complexos. Isso funciona muito bem com sinais simulados e sem ruído, porém em sinais reais o comportamento da resolução espectral com a presença de ruído ainda precisa ser melhor analisada. ${ }^{104}$

Na Fig. 5.19 A) os dois dubletos na região central apresentam separação de 2,5 Hz. Em C) e D) usando $T_{p}=100 \mathrm{~ms}$, temos que a SSFPdx e SSFPdxdt não apresentam resolução na separação desses dubletos. A separação dos dubletos em $2,5 \mathrm{~Hz}$ corresponde a um batimento no eixo dos tempos com frequência de período 0,4 s. Uma vez que nossa aquisição durou apenas 0,1 segundos, a informação desse dubleto provavelmente foi perdida na aquisição, de modo que a Transformada de Fourier não obteve resolução. Os outros picos estão espaçados de $14,2 \mathrm{~Hz}$, que corresponde a frequência de período $0,07 \mathrm{~s}$; sendo completamente resolvidos no sinais SSFP.

Para avaliar se a perda de resolução foi devido a ausência de um período do batimento na aquisição, a Fig. $5.19 \mathrm{E}$ ) apresenta o sinal SSFPdxdt adquirido com $T_{p}=0,2 \mathrm{~s}$ processado com a Transformada de Fourier, e F) processado o mesmo sinal com o KBDM, onde ainda não há resolução dos dubletos. Na Fig. 5.19 G) o sinal SSFPdxdt foi adquirido com $T_{p}=0,4$ $\mathrm{s}$, completando a aquisição de um período do batimento, e vemos que os dubletos se tornam visíveis tanto na Transforma de Fourier em G) quando no processamento pelo KBDM em H).

Esses resultados mostram que o processamento com o KBDM 'limpou' o espectro, removendo ruído e melhorando a linha de base, porém não se apresentou mais eficiente que a Transformada de Fourier com função de apodização e zero-filling na obtenção de resolução espectral além do limite de resolução imposto na DFT. 


\section{Capítulo 6}

\section{Diagonalização Filtrada e Inversa de Laplace}

Without computers, no modern NMR.

Richard R. Ernst

As técnicas de processamento digital de sinais tem importante papel na RMN moderna, sendo indispensáveis em inúmeras aplicações, como na obtenção dos espectros de frequências pela Transformada de Fourier, deconvolução de picos, eliminação de sinais espúrios ou solvente, aumento de resolução ${ }^{105}$, ou ainda separação das componentes de decaimentos exponenciais pela Transformada Inversa de Laplace. ${ }^{106}$

Neste capítulo descrevemos os trabalhos realizados com o Método da Diagonalização Filtrada (FDM) em sua versão denominada Krylov Basis Diagonalization Method (KBDM) e com a Transfomada Inversa de Laplace (ILT). Iniciamos apresentando o desenvolvimento do KBDM para análise de sinais SSFP em alta resolução a fim de obter melhor qualidade espectral do sinais adquiridos, posteriormente apresentamos a versão modificada do algorítimo KBDM como uma via alternativa a Transformada Inversa de Laplace na construção de diagramas de distribuição de tempos de relaxação $T_{2}$. Esses trabalhos foram realizados em parceria com o Prof. Dr. Cláudio José Magon, do Instituto de Física de São Carlos da USP. 


\subsection{KBDM para supressão das anomalias na SSFP}

A descrição matemática do Método da Diagonalização Filtrada pode ser encontrada na dissertação de mestrado do autor ${ }^{90}$ assim como os detalhes da versão KBDM no artigo ${ }^{51}$, sendo portanto omitidas nesta tese.

Os dois principais fatores para medir a qualidade de um espectro são a resolução e sensibilidade do mesmo. Resolução se refere a capacidade de distinguir componentes de frequências próximas, e sensibilidade é a capacidade de distinguir sinais dos ruídos, tipicamente pelo parâmetro da razão $s / r$.

O processamento de sinais SSFP com a Transformada de Fourier resulta em espectros com fortes batimentos e anomalias de fase e amplitude. Os batimentos laterais são causados devido ao forte truncamento dos sinais SSFP e as anomalias de fase e amplitude devido a presença da componente eco, como discutido no capítulo 5 . Os batimentos podem ser facilmente suprimidos através da utilização de funções de apodização especiais como por exemplo a função Traf. ${ }^{51}$ Já as anomalias de fase e amplitude são geradas pela presença da componente eco nos sinais, de modo que inicialmente o processamento com métodos paramétricos foi proposto com o intuito de ao mesmo tempo remover os batimentos laterais e deconvoluir a componente eco dos sinais SSFP, para obter espectros com melhor qualidade.

No artigo ${ }^{51}$ apresentamos essa proposta processando sinais de ${ }^{13} \mathrm{C}$ de Brucina dissolvida em DMSO- $d_{6}$. Foram realizadas aquisição SSFP (sem alternância de fase), processadas pela KBDM e pela Transf. de Fourier, para comparação com os espectros com aquisição convencional (zgpg30).

A Fig. 6.1 apresenta a comparação entre o sinal FID de ${ }^{13} \mathrm{C}$ Brucina adquirido em A) com a sequência de pulsos convencional com tempo de aquisição $1,3 \mathrm{~s}$ e tempo de reciclo $t_{r}=1 \mathrm{~s}$. Fig. $6.1 \mathrm{~B}$ ) sinal adquirido com a SSFP com $100 \mathrm{~ms}$ de aquisição, $t_{r}=10 \mu \mathrm{s}$, resultando em 1242 pontos complexos. O FID SSFP apresenta as características já discutidas na seção 5.1 prevalecendo seu formato truncado com componentes decrescentes (FID) e crescentes (eco).

O processamento pelo KBDM gera um lista (line list) com os parâmetros $\left\{f_{k}, d_{k}, \theta_{k}, \gamma_{k}\right\}$ sendo a frequência, amplitude, fase e largura de cada componente $k$ espectral. Através dessa lista de parâmetros podemos separar as componentes ecos do sinal, pois essas são caracte- 


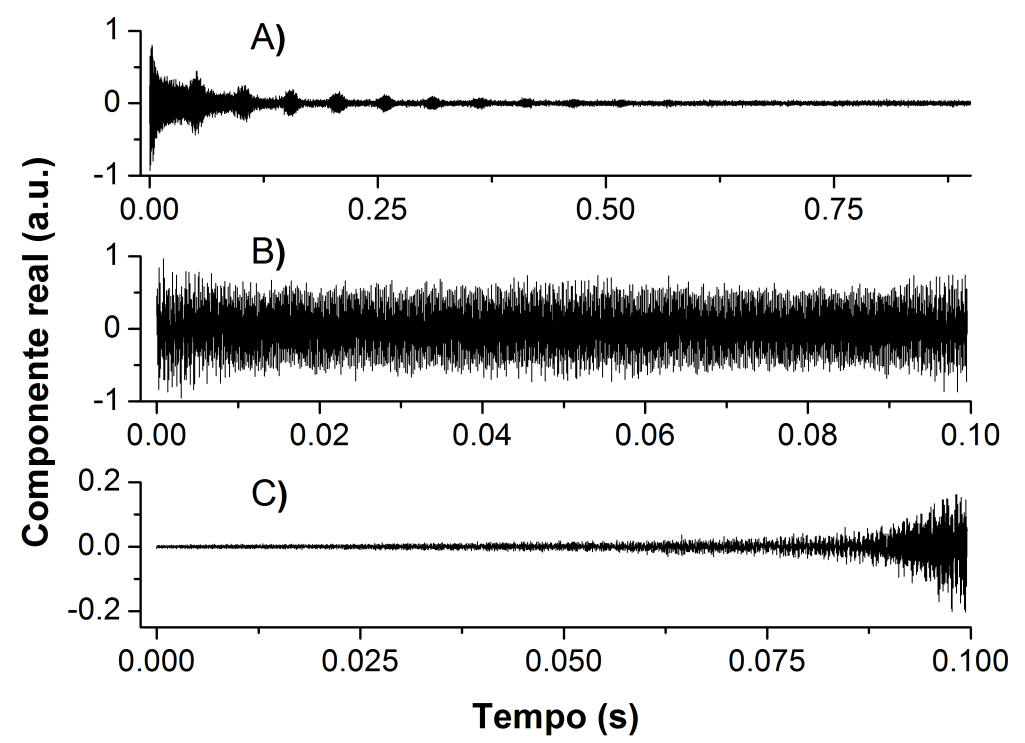

Figura 6.1- Componente real do FID obtido com a sequência de pulsos A) convencional (zgpg30) e B) SSFP com 100 ms de aquisição. Em C) apresentamos a componente eco retirada do sinal SSFP através do processamento com o KBDM.

Fonte: Elaborada pelo autor.

rizadas por apresentar exponenciais crescentes, ou seja $\gamma_{k}$ negativo. Essa deconvolução da componente eco é apresentada na Fig. 6.1 C).

A Fig. 6.2 apresenta o espectro ${ }^{13} \mathrm{C}$ da Brucina obtido pela Transformada de Fourier do FID em Fig. 6.1 A). Além da visualização do espectro total entre 0 e 220 ppm, apresentamos duas regiões com forte densidade de picos expandidas. A primeira centrada em 30 ppm apresenta o pico do solvente DMSO- $d_{6}$ e a segunda entre 40 e 65 ppm uma região de alta densidade de picos da Brucina. Esse espectro padrão foi adquirido para ser base de comparação com os sinais adquiridos com a sequências de pulsos SSFP utilizando 100 ms, 50 ms e 25 ms no tempo de aquisição, e posteriormente processados com a Transformada de Fourier e com o KBDM.

Na Fig. 6.3 são apresentados os espectros adquiridos com a SSFP com tempo de aquisição de 100 ms, 50 ms e 25 ms processados com a Transformada de Fourier (à esquerda) e pelo KBDM (à direita) nas duas regiões de alta concentração de picos. A Transformada de Fourier foi utilizada com uma função de apodização Traf (10) e zero-filling de 32768 pontos. Na coluna à esquerda vemos que os espectros processados com a TF com função Traf removeu parte das anomalias de truncamento, porém com baixa resolução e anomalias na amplitude.

Os espectros Fig. 6.3 a'), b') e c') apresentam os mesmos sinais adquiridos respectivamente 


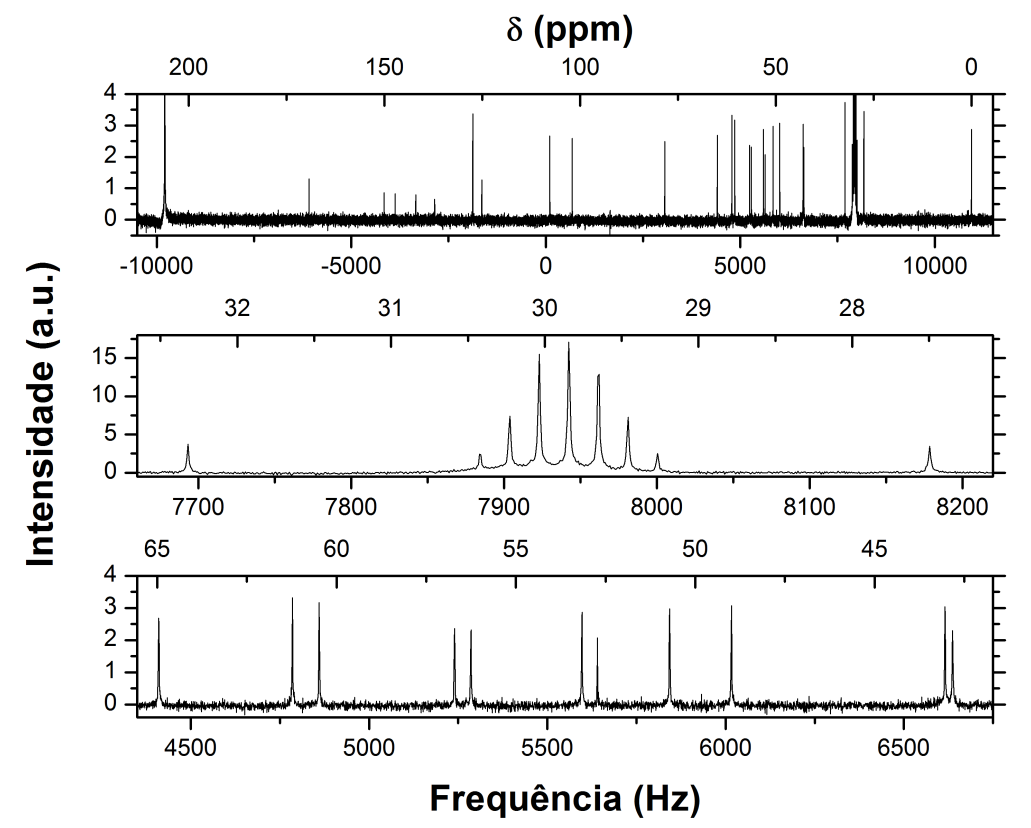

Figura 6.2- Componente absortiva do espectro de Fourier de ${ }^{13} \mathrm{C}$ da Brucina/DMSO- $d_{6}$ adquirida com a sequência de pulsos convencional (zgpg30) e tempo total de experimento de 90 minutos.

Fonte: Elaborada pelo autor.

com a SSFP de $100 \mathrm{~ms}, 50 \mathrm{~ms}, 25 \mathrm{~ms}$ e processados com o KBDM. Em geral, os espectros KBDM reproduziram muito bem o espectro apresentado na Fig. 6.2, com resolução suficiente para resolver os 23 carbonos da Brucina. Até mesmo o par de picos em 43 ppm foi resolvido. Entretanto, a intensidade dos picos não foi corretamente reproduzida, devido a natureza da interação FID e eco nos sinais SSFP.
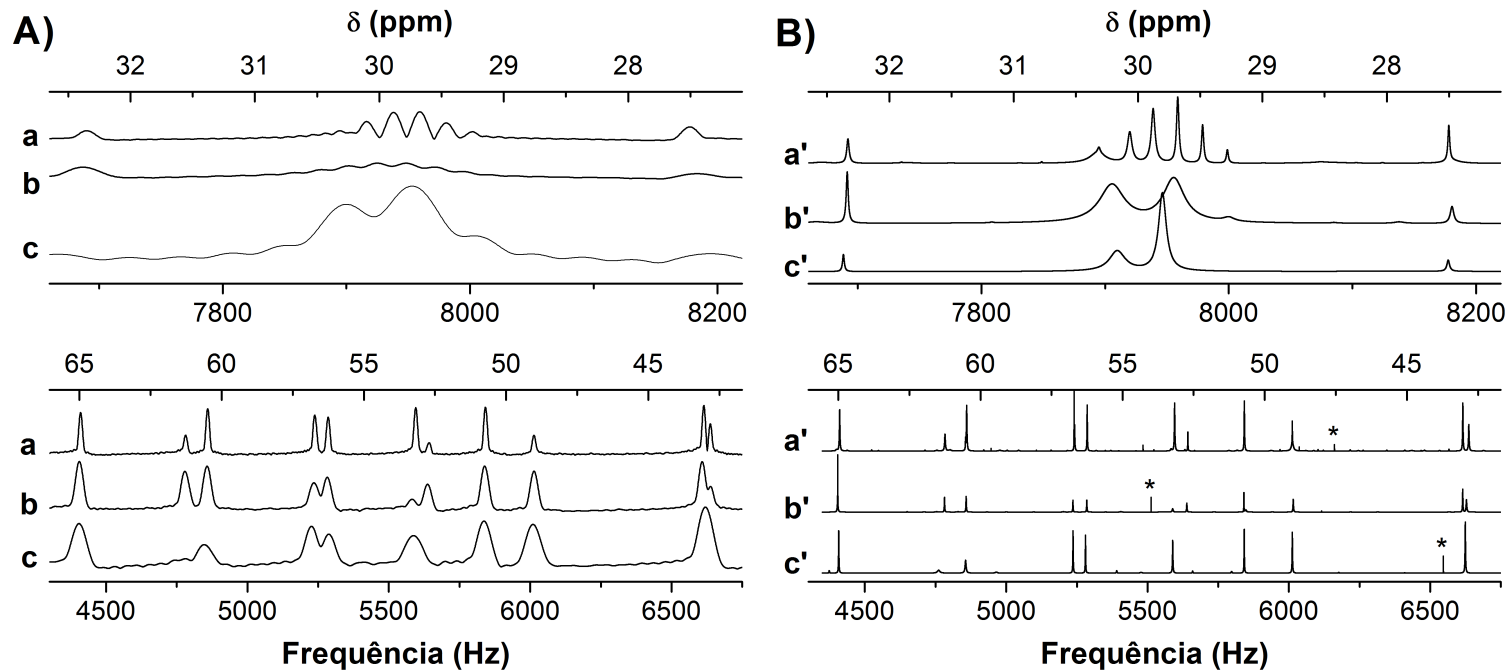

Figura 6.3- Espectros de ${ }^{13} C$ Brucina adquiridos com SSFP com tempos de aquisição de a) $100 \mathrm{~ms}$, b) $50 \mathrm{~ms}$ e c) $25 \mathrm{~ms}$. Em A) apresentamos o espectro gerado pelo processamento com a Transformada de Fourier com função Traf expandindo duas regiões do espectro. Em B) comparamos os espectros gerados pelo processamento do mesmo sinal SSFP com o método KBDM.

Fonte: Elaborada pelo autor. 
Como esperado, o processamento pelo KBDM removeu os batimentos e anomalias de fase. Boa resolução foi encontrada para as aquisições SSFP com 100 ms e 50 ms, enquanto 25 ms não resolveu uma série de picos com deslocamentos químicos próximos ao longo do espectro. Na expansão a')-c') do solvente na Fig. 6.3 nota-se que foram resolvidos 6 picos, ao invés dos 7 determinados na FT da aquisição padrão. Isso mostra que o KBDM obteve resolução superior a FT, porém não foi capaz de promover completa resolução nessa situação. Os asteriscos $(*)$ marcados em Fig. 6.3 indicam picos espúrios do processamento pelo KBDM, pois ao reprocessar o sinal com pequena variação nos parâmetros de entrada, esses picos não são reproduzidos.

Por fim, esses resultados mostram a eficiência do KBDM em processar sinais de ${ }^{13} \mathrm{C}$ SSFP. Esses resultados e mais detalhes são descritos com detalhes no artigo. ${ }^{51}$

\section{SSFP com alternância de fase}

Na primeira etapa desse estudo vimos que o processamento com o KBDM consegue deconvoluir a componente eco nos sinais SSFP, entretanto, isso não garantiu a completa eliminação dos efeitos adversos observados na SSFP. A segunda estratégia elaborada para esse problema é suprimir a componente eco durante a aquisição dos sinais SSFP e então processa-lo com o KBDM.

Os métodos desenvolvido para supressão das componentes ecos na SSFP foram descritos no capítulo 5 e são baseados em sequências SSFP com esquemas de alternância ou incremento de fase. Assim, nesta seção apresentaremos sinais adquiridos com as sequências SSFPdx e SSFPdxdt e comparamos seu processamento com o KBDM e a Transformada de Fourier.

A Fig. 6.4 apresenta a comparação dos espectros de ${ }^{13} \mathrm{C}$ da Brucina/DMSO- $d_{6}$ adquirida com a SSFP sem alternância de fase, processado com a Transformada de Fourier e com o KBDM. Nesta situação, a interação destrutiva entre as componentes FID e eco resultam na supressão na região central do espectro mostrado, de modo que mesmo com a deconvolução pelo processamento com o KBDM, o espectro resultante não apresenta todos os picos. Esse resultado evidência a necessidade de se estabelecer métodos que suprimam o sinal eco durante a aquisição do sinal SSFP. 


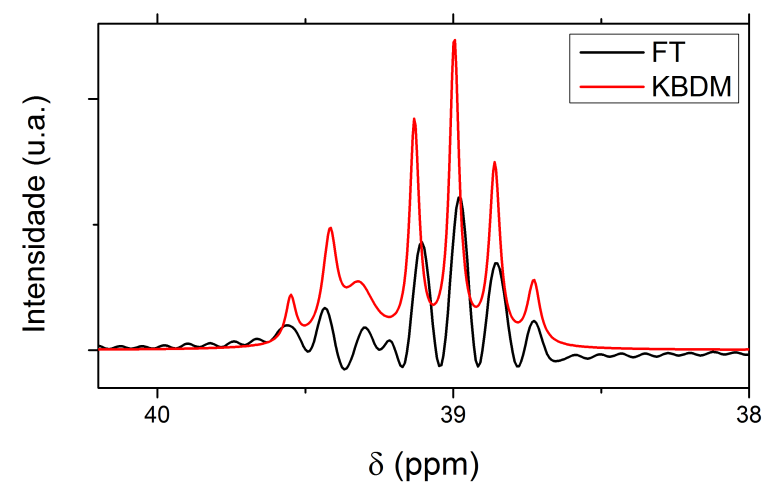

Figura 6.4- Sinais ${ }^{13} C$ SSFP sem alternância de fase da o solvente DMSO- $d_{6}$, processados com a Transformada de Fourier e com o KBDM.

Fonte: Elaborada pelo autor.

Na Fig. 6.5 apresentamos espectros $d e{ }^{13} C$ da Brucina/DMSO- $d_{6}$ adquirida com a SSFPdx com 8 blocos de aquisição, processados com a Transformada de Fourier e com o KBDM. Temos agora que em ambos os processamentos, o espectro se apresenta completo, uma vez que a interação destrutiva entre FID e eco foi resolvida no processo de aquisição dos sinais SSFP. Vale destacar ainda que com o KBDM anomalias de fase e amplitude são reduzidas, além da eliminação de ruídos da linha de base.
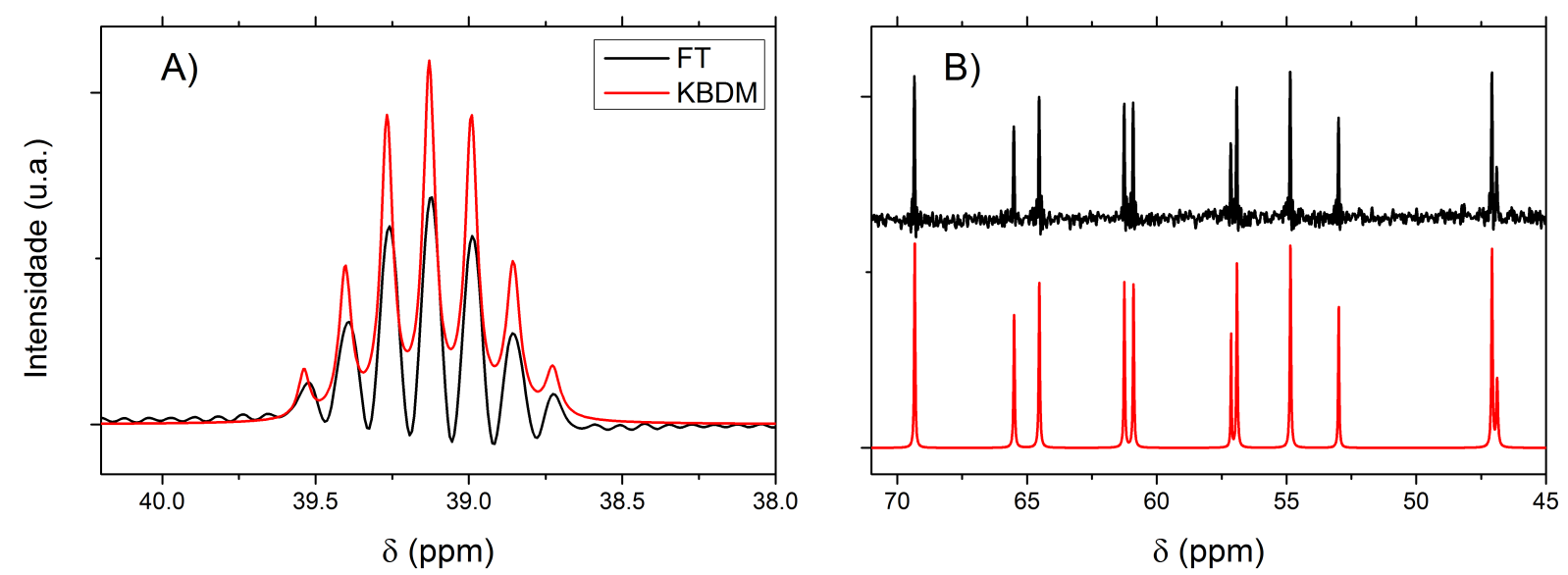

Figura 6.5- Regiões do espectros de ${ }^{13} C$ SSFPdx com 8 blocos de aquisição da amostra Brucina/DMSO- $d_{6}$, processados com a Transformada de Fourier e com o KBDM.

Fonte: Elaborada pelo autor.

A segunda técnica aplicada foi a SSFPdxdt, com incremento não-linear de fase. Na Fig. 6.6 apresentamos regiões do espectro da Brucina/DMSO- $d_{6}$, adquirido agora com $T_{p}=200 \mathrm{~ms}$ e com incremento de fase $\pi / 256$. Tanto a Transformada de Fourier quando o KBDM resolvem bem esse espectro, com a vantagem no KBDM de melhor ajuste de fase dos picos e 'limpar' os pequenos ruídos na linha de base do espectro. 

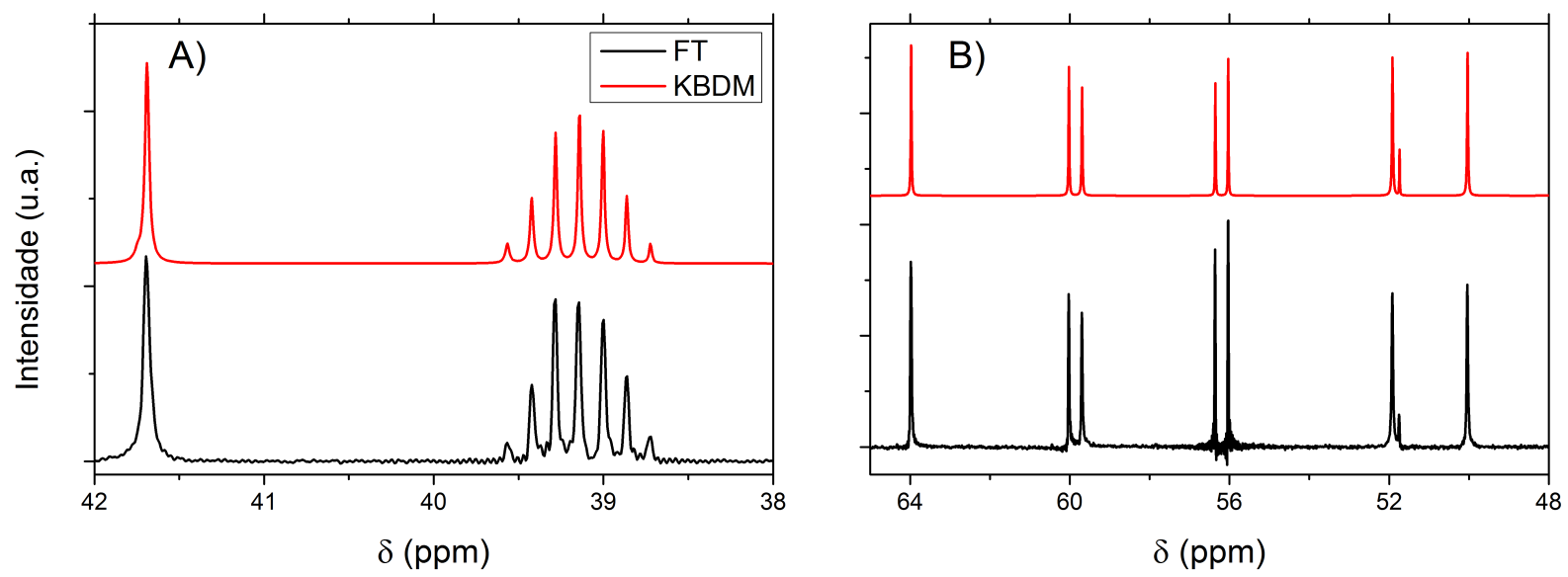

Figura 6.6- Regiões do espectros de ${ }^{13} C$ SSFPdxdt com incremento $\pi / 256$ da amostra Brucina/DMSO- $d_{6}$ com $T_{p}=200 \mathrm{~ms}$, processados com a Transformada de Fourier e com o KBDM.

Fonte: Elaborada pelo autor.

Desse modo, unindo as aquisições com as sequências SSFPdx-n8 ou SSFPdxdt com o processamento do sinal através do KBDM ou FDM, a supressão espectral é evitada pelo novo esquema de aquisição, e os batimentos sinc e anomalias de fase observados na Transformada de Fourier são corrigidas pelos KBDM, além de melhorar a razão $s / r$ dos espectros.

\subsection{Distribuição de tempos de relaxação}

Medidas de RMN dos tempos de relaxação $\left(T_{1}\right.$ e $\left.T_{2}\right)$ e coeficiente de difusão (D) são ferramentas poderosas para estudo dos líquidos localizados no interior de meios porosos. Nesse contexto, a RMN vem sendo intensivamente utilizada em estudos de ciência básica e aplicada, como em áreas da engenharia e química, na indústria de alimentos (Food Analysis), ciência dos materiais e no setor petrolífero (Oil Science). ${ }^{84}$

Um objetivo comum na análise experimental de dados em experimentos de baixa resolução é a determinação dos tempos de relaxação ou de coeficiente de difusão por métodos de fitting exponencial, que nos casos de sinais ruidosos, consiste em um problema matemático mal-posto, não possuindo solução única. ${ }^{89,107}$

Em situações com uma ou duas componentes é comum a determinação através de métodos de fitting mono ou bi-exponencial, porém em sistemas mais complexos é necessária a utilização de métodos numéricos da Transformada Inversa de Laplace, determinando assim uma distribuição de tempos de relaxação. A Inversa de Laplace é principalmente utilizada na determinação 
da distribuição de tempos de relaxação $T_{2}$ de experimentos CPMG, que através da utilização de métodos de regularização, vínculos são introduzidos forçando uma solução para um determinado parâmetro $\alpha .{ }^{107}$ A Fig. 6.7 apresenta um esquema do emprego da Transformada Inversa de Laplace em um sinal CPMG composto por uma soma de decaimentos exponenciais.
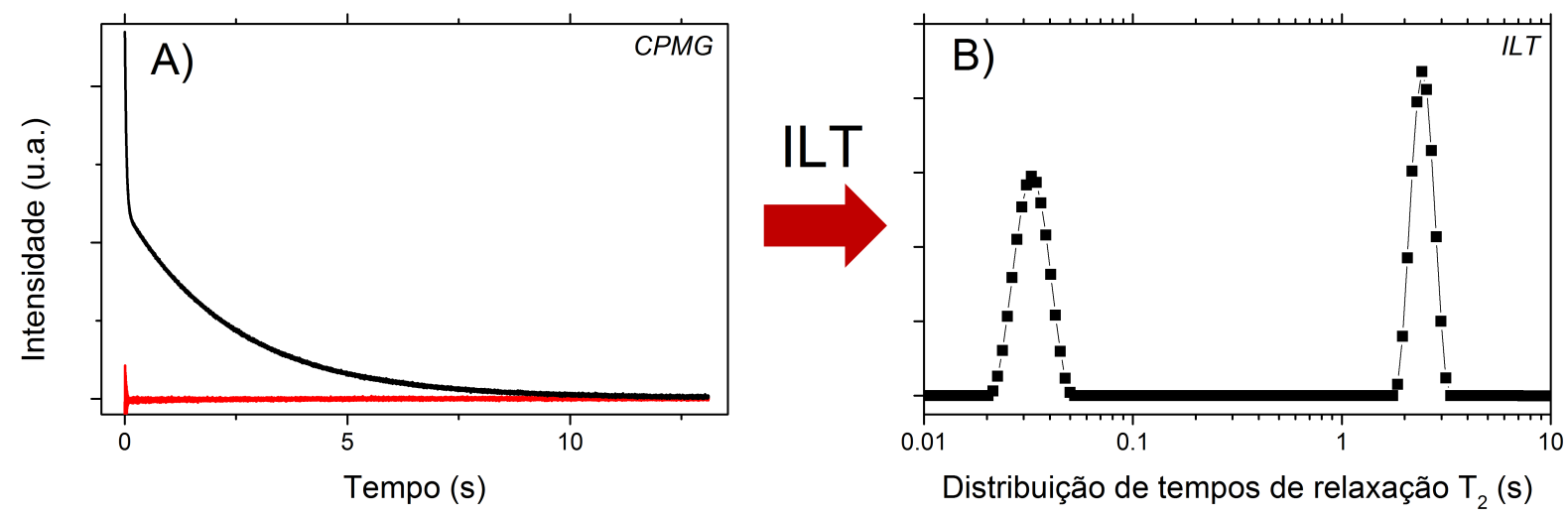

Figura 6.7- Um sinal composto pela soma de decaimentos exponenciais é um problema matemático malposto, pois mais de uma solução é possível. A Transformada Inversa de Laplace em conjunto com métodos de regularização, como o SVD e Tikhonov, forçam uma solução de acordo com um parâmetro $\alpha$ escolhido, resultando na distribuição de tempos como em B).

Fonte: Elaborada pelo autor.

Um ponto crítico comum na maioria dos métodos de regularização da inversão de Laplace é a resolução espectral obtida. Por exemplo, dado um pico único na distribuição, a largura de linha representa a descrição física do experimento ou é uma característica da regularização realizada? Outra pergunta relacionada é: o quão distante dois picos devem estar de modo a serem bem resolvidos? Essas questões são exploradas em diversos artigos recentes, que introduzem diversas formas de regularização ${ }^{107}$, buscam calcular a otimização do parâmetro $\alpha$ e mostram que a resolução é fortemente dependente da razão $s / r$, dentre outros parâmetros experimentais. Os métodos de regularização utilizados nesta tese são a regularização de Tikhonov ${ }^{91}$ e Decomposição de Valores Singulares (Singular Values Decomposition - SVD). ${ }^{89}$

Nesta seção, apresentamos o desenvolvimento de um método alternativo a Transformada Inversa de Laplace, baseada no Método da Diagonalização Filtrada, focando em sua utilização na determinação da distribuição de tempos de relaxação $T_{2}$ oriundos de experimentos CPMG. Resultados com soluções aquosas de $\mathrm{CuSO}_{4}$, óleos, alimentos, cerâmicas e rochas mostram que é possível obter resultados compatíveis aos métodos da Transforma Inversa de Laplace. Esses estudos foram realizados em conjunto com o Prof. Dr. Cláudio J. Magon, e o aluno de 
doutorado E.T. Montrazi orientado do Prof. Dr. Tito J. Bonagamba do Instituto de Física de São Carlos, USP.

De modo a avaliar o método proposto, experimentos CPMG foram processados e extensivamente comparado com o algoritmo mundialmente popularizado de Inversão de Laplace non-negative least-squares (NNLS) ${ }^{89}$, com regularização de Tikhonov e SVD. Na primeira seção apresentamos o algorítimo da ILT-NNLS implementado em Matlab e na seção seguinte introduzimos a análise exponencial com o KBDM e comparação dos resultados.

\subsubsection{Transformada Inversa de Laplace}

Historicamente o primeiro método numérico que resolveu Transformada Inversa de Laplace satisfatoriamente, foi o algoritmo CONTIN, no começo da década de $80 .{ }^{10}$ Posteriormente, diversos algoritmos e métodos de regularização foram propostos, entre os mais famosos podemos citar o non-negative least-squares (NNLS) e o método de Butler-Reeds-Dawson (BRD), com a introdução de métodos rápidos de processamento na Laplace bi-dimensional. ${ }^{82,83}$

Transformada Inversa de Laplace são métodos numéricos muito utilizados na determinação da distribuição de tempos de relaxação ou coeficiente de difusão na RMN. ${ }^{89,107}$ Um típico experimento de relaxação resulta em sinais que decaem no tempo, como por exemplo, sinais de CPMG de líquidos geram um decaimento exponencial, onde $c$ é o sinal experimental, $t$ é período de tempo do sinal adquirido e $T_{2}$ a constante de relaxação. Para uma amostra composta por vários tempos de relaxação

$$
c(t)=\sum_{k} c_{k}(0) \exp \left(-t / T_{2, k}\right)
$$

onde $k$ denota o número de componentes presente na amostra e $T_{2, k}$ a constante $T_{2}$ da $k$-ésima componente. Assumindo a existência de uma distribuição contínua de tempos de relaxação, podemos escrever o sinal como sendo a integral

$$
c(t)=\int g\left(T_{2}\right) \exp \left(-t / T_{2}\right) d T_{2}
$$

onde a função $g\left(T_{2}\right)$ representa a distribuição dos tempos $T_{2}$, fornecendo as amplitudes de 
cada componente infinitesimal $d T_{2}$. O propósito da Transformada Inversa de Laplace é obter a distribuição de amplitudes $g\left(T_{2}\right)$, a partir do dado experimental $c(t)$.

Para um sinal CPMG experimental, Fig. 6.7, um conjunto discreto de dados $n$ é adquirido no intervalo de tempo $t$, com a presença do ruído experimental $\left(\epsilon_{n}\right)$

$$
c(t)=\sum_{j=1}^{m} g\left(T_{2, j}\right) K\left(t_{n}, T_{2, j}\right)+\epsilon_{n}
$$

onde $\mathrm{K}$ é o núcleo (kernel) dessa somatória. Para os estudos aqui apresentados definimos nosso kernel como sendo composto por exponenciais decrescente. No caso de decaimentos exponenciais

$$
K=\left(\begin{array}{cccc}
e^{-t_{1} / T_{1}} & e^{-t_{1} / T_{2}} & \cdots & e^{-t_{1} / T_{m}} \\
e^{-t_{2} / T_{1}} & e^{-t_{2} / T_{2}} & \cdots & e^{-t_{2} / T_{m}} \\
\vdots & & \ddots & \vdots \\
e^{-t_{n} / T_{1}} & e^{-t_{n} / T_{2}} & \cdots & e^{-t_{n} / T_{m}}
\end{array}\right)
$$

com dimensão $n \times m$ onde $n$ é a dimensão do vetor de dados CPMG e $m$ a dimensão escolhida para o espectro de distribuição $T_{2}$ resultante.

A inversão desse problema é obtida através da minimização dos erros quadráticos, dada por

$$
\chi^{2}=\|c(t)-F(t)\|^{2}
$$

onde $F(t)$ é a função que melhor descreve os dados experimentais. Esse problema de minimização não é trivial, pois se trata de um problema matemático mal-posto, admitindo mais de uma solução.

\section{Regularização}

Os métodos de regularização são baseados na ideia de forçar uma solução da inversão estabelecendo pressupostos da solução esperada. Assim, em experimentos em meios porosos, fisicamente esperamos obter uma distribuição contínua de distribuição de tempos de relaxação, sem a existência de picos delta, pontos descontínuos ou valores negativos na distribuição. ${ }^{89}$

Essa ideia de distribuição contínua de tempos pode ser imposta com a regularização de 
Tikhonov ${ }^{89,91}$, minimizando a função

$$
\chi^{2}=\left\{\|c(t)-F(t)\|^{2}+\alpha\|\vec{g}\|^{2}\right\}
$$

onde o termo acrescentado representa a penalização imposta com vínculo de intensidade $\alpha$. Essa penalização pode ser construída de diversas formas, estabelecendo uma ligação entre as componentes $g_{i}$ da função.

Além da suavização da distribuição com o termo $\alpha$, também impomos a determinação de componentes não-negativas e valores com amplitude nula nas bordas do espectro de distribuição $T_{2}$. Assim as distribuições obtidas se tornam mais estáveis e contínuas e o termo $\alpha$ é responsável pela suavização da solução. A escolha do termo de regularização $\alpha$ é subjetiva e diversos métodos se propõe a sua otimização, todas com ótimo ajuste de fitting do decaimento.

Para fins de comparação com método do KBDM, foi desenvolvido um pacote no Matlab - Apêndice 8 - que resolve a Transformada Inversa de Laplace pelo método mundialmente utilizado non-negative least-squares (ILT-NNLS) com regularização de Tikhonov e Decomposição de Valores Singulares (SVD). O algoritmo foi desenvolvido seguindo de perto o descrito na referência. ${ }^{89}$

\subsubsection{Análise exponencial via KBDM}

O Método da Diagonalização Filtrada é um método numérico introduzido em 1995 na literatura por Wall e Neuhauser ${ }^{108}$, onde mostraram que com apenas a função de correlação temporal $c(t)$ é possível calcular os autovalores e autovetores de uma pequena faixa de energia de um sistema quântico de grande dimensão. Essa formulação do método é extremamente adequada para a análise espectral de sinais temporais, entretanto sua implementação numérica inicial foi muito ineficiente e permaneceu apenas como uma curiosidade interessante.

Em 1998 Mandelshtam estendeu o método utilizando um filtro retangular ${ }^{109}$, onde finalmente conseguiu-se convergência, velocidade e confiabilidade e introduziu-se o nome Filter Diagonalization Method (FDM). O FDM passou a ser explorado em diversas áreas da Física 
e Química onde a inversão harmônica é necessária. Em particular, o FDM começou a ser utilizado para RMN passando a ser uma das mais interessantes aplicações do método. Uma excelente descrição do algoritmo do FDM foi detalhada num artigo de revisão por Mandelshtam em $2001^{110}$, onde cita os principais trabalhos desenvolvidos na época e da ênfase às aplicações do FDM na área da Ressonância Magnética Nuclear.

Uma versão reduzida do FDM é o KBDM (Krylov Basis Diagonalization Method). ${ }^{51}$ Em essência ambos os métodos resultam nos mesmos resultados, entretanto o FDM possui uma série de parâmetros que devem ser ajustados por um espectroscopista especializado. Visando retirar essa dependência do operador, nosso grupo vem desenvolvendo trabalhos também com o KBDM, que não possui esses parâmetros de processamento, mas é um Black Box, retornando o mesmo resultado independente do operador. A desvantagem do KBDM se estabelece para sinais muito longos, onde o tempo de execução da rotina pode tornar a análise inviável.

Os primeiros estudos no Brasil com o FDM foram realizados pelo Prof. Dr. Cláudio J. Magon, do Instituto de Física de São Carlos - USP, sendo inicialmente utilizado para análises de sinais na Ressonância Paramagnética Eletrônica EPR. Posteriormente diversas versões foram implementadas para análises de sinais espectrais de RMN em alta resolução, como a método FDM-Multi Scale, com regularização de Tikhonov (FDM2K) e Decomposição Valores Singulares (SVD). ${ }^{90}$

Dentro desse contexto, surgiu a ideia da utilização do FDM e/ou do KBDM para análise de sinais de CPMG de RMN e baixa resolução. O KBDM é um método numérico que extrai os parâmetros de um sinal temporal composto por somatórias de exponenciais oscilatórias amortecidas

$$
c_{n}=c\left(t_{n}\right)=\sum_{k=0}^{K-1} d_{k} e^{i n \omega_{k} \tau}+\epsilon_{n}
$$

onde $t_{n}=n \tau$, com $n=0,1, \ldots, N-1$, com amplitudes complexas $d_{k}=\left|d_{k}\right| e^{i \theta_{k}}$ e frequências complexas $\omega_{k}=2 \pi f_{k}+i \gamma_{k}$, onde $\theta_{k}$ são as fases $\gamma_{k}$ as constante de amortecimento (com $\left.T_{2, k}=1 / \gamma_{k}\right), \epsilon_{n}$ é o ruído experimental complexo e $\tau$ o tempo entre pontos.

Esses parâmetros $\left\{\left|d_{k}\right|, \theta_{k}, f_{k}, \gamma_{k}\right\}$ carregam a informação física do sinal estudado e sua avaliação a partir dos dados experimentais é um problema matemático fundamental conhecido como Inversão Harmônica (Harmonic Inversion Problem - HIP). Esse problema é não linear e 
pode ser convertido num problema de autovalores generalizado (General Eigenvalue Problem - GEP) por pura álgebra linear. ${ }^{90}$

$$
U^{(1)} B_{k}=\mu_{k} U^{(0)} B_{k}
$$

Resolvida essa equação de autovalores generalizada, a lista de parâmetros $\left\{\left|d_{k}\right|, f_{k}, \theta_{k}, \gamma_{k}\right\}$ é determinada, onde os autovalores $\left\{\mu_{k}\right\}$ dessa equação fornecem as frequências e constantes de amortecimento $\left(2 \pi f_{k}+i \gamma_{k}\right)$ e os autovetores $\left\{B_{k}\right\}$ as amplitudes e fase do sinal $\left|d_{k}\right| e^{i \theta_{k}}$, através das relações

$$
\left\{\begin{array}{l}
\mu_{k}=e^{i \tau \omega_{k}} \\
\sqrt{d_{k}}=B_{k}^{T} C
\end{array}\right.
$$

onde $B_{i}^{T} U^{0} B_{j}=\delta_{i j}$ e o vetor $C=\left(c_{0}, c_{1}, \cdots, c_{K-1}\right)^{T}$.

A construção das matrizes $U^{(0)}$ e $U^{(1)}$ são feitas diretamente com os dados do sinal experimental $c(t)$ e dependem do método a ser utilizado. No KBDM as matrizes são construídas da forma $\left[U^{p}\right]_{i, j}=c_{i+j+p}$, com dimensão $m \times m$ onde $m=n / 2$.

$$
U^{0}=\left(\begin{array}{cccc}
c_{0} & c_{1} & \cdots & c_{m-1} \\
c_{1} & c_{2} & \cdots & c_{m} \\
\vdots & \vdots & \ddots & \vdots \\
c_{m-1} & c_{m} & \cdots & c_{2 m-2}
\end{array}\right) ; U^{1}=\left(\begin{array}{cccc}
c_{1} & c_{2} & \cdots & c_{m} \\
c_{2} & c_{3} & \cdots & c_{m+1} \\
\vdots & \vdots & \ddots & \vdots \\
c_{m} & c_{m+1} & \cdots & c_{2 m-1}
\end{array}\right)
$$

Os sinais adquiridos com a sequência de pulsos CPMG resultam numa somatória de decaimentos exponenciais de frequência nula. A análise das exponenciais presentes nesse decaimento através do KBDM gera uma lista de parâmetros com diversos valores discretos de $\left|d_{k}\right|, f_{k}, \theta_{k}$, $\gamma_{k}$, onde o tempo de relaxação $T_{2}$, são dados por

$$
T_{2, k}=\frac{1}{\pi \gamma_{k}}
$$

com suas respectivas amplitudes $d_{k}$. Esse procedimento retorna uma distribuição discreta de tempos de relaxação, como apresentado na Fig. 6.11. De modo a gerarmos a distribuição de tempos $T_{2}$, implementamos o KBDM com o método de pseudo-noise averaging, que intro- 
duz um parâmetro de ruído branco denominado (PNA), calculado como uma porcentagem, tipicamente $2 \%$, do RMS (root mean square) do ruído do sinal CPMG.

Através da introdução do parâmetro PNA no sinal CPMG original e realização de um número suficiente de médias computacionais, o KBDM nos retorna uma distribuição comportada de tempos de relaxação $T_{2}$, como explicado no esquema Fig. 6.11.
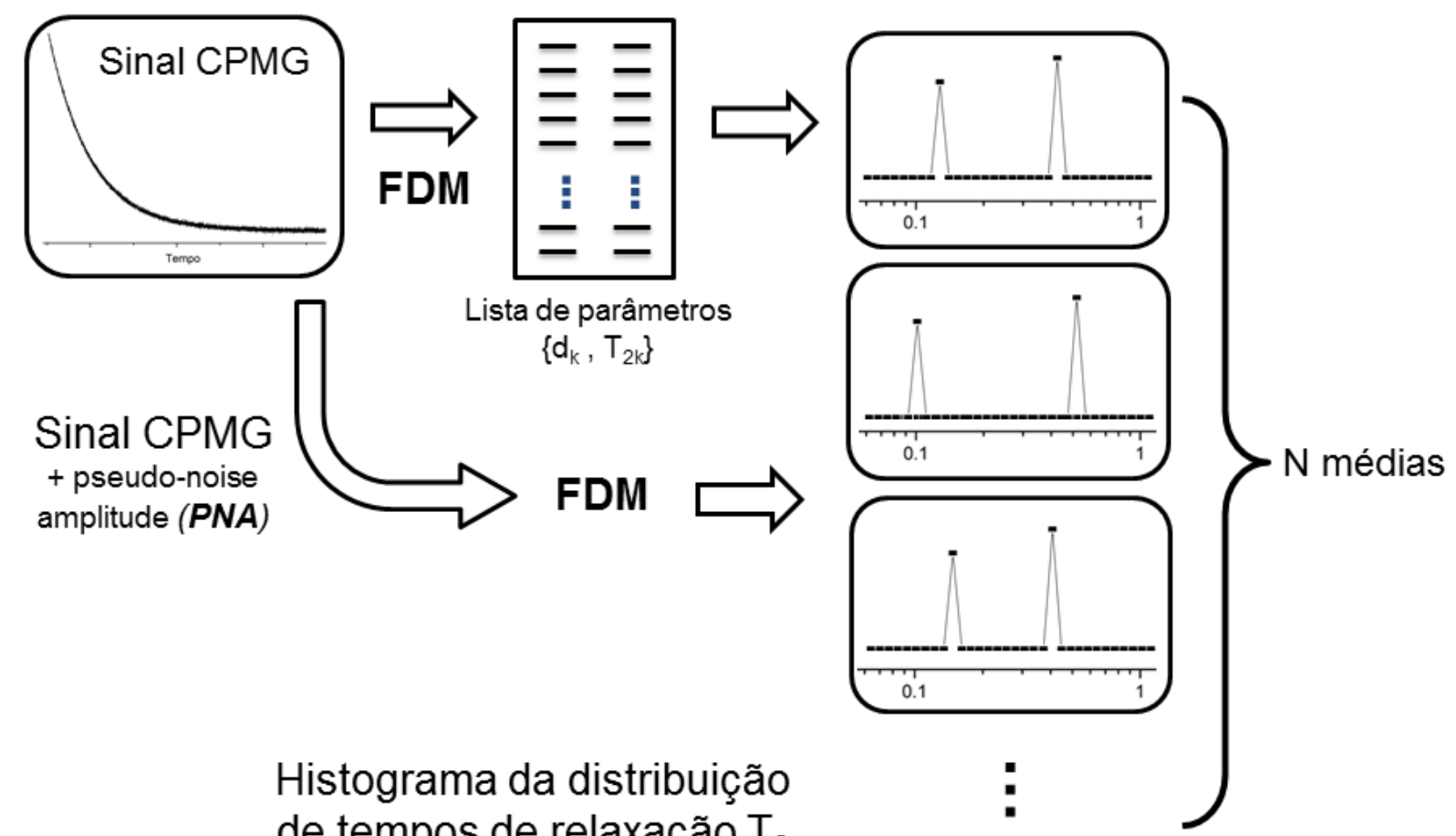
de tempos de relaxação $T_{2}$
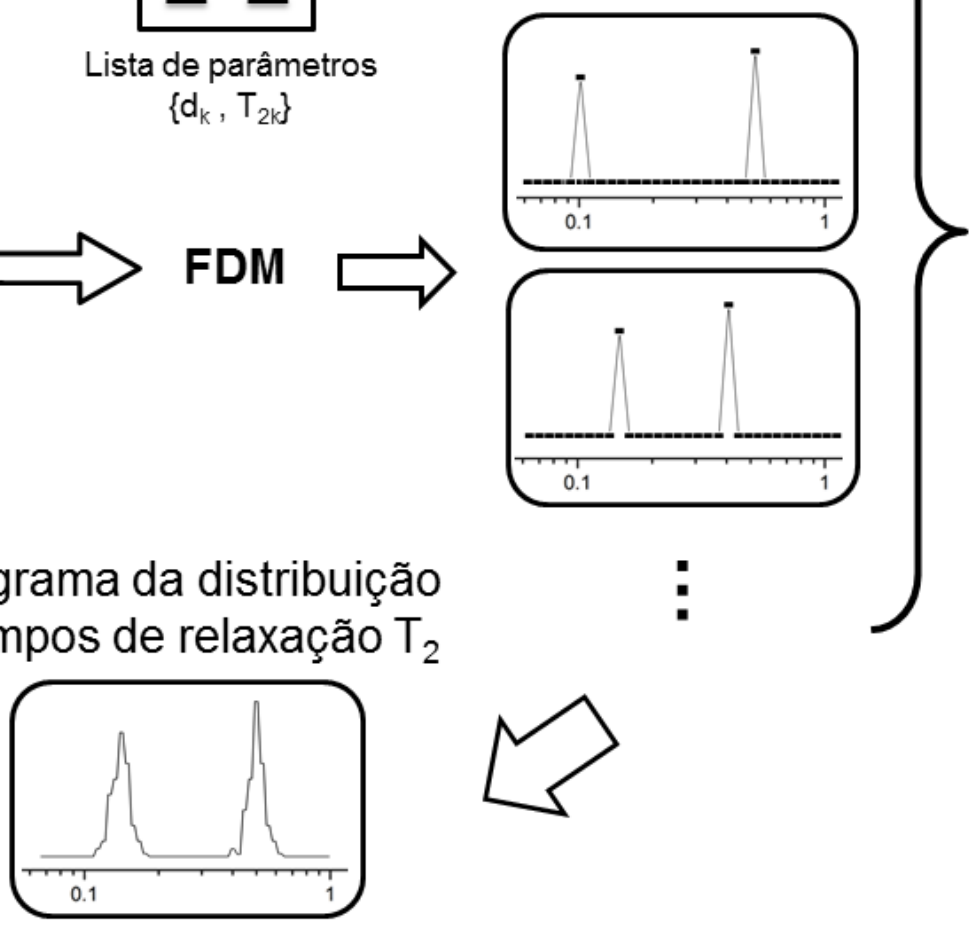

Figura 6.8- Esquema do método KBDM para obtenção de distribuição de tempos de relaxação. Através da introdução do ruído branco (PNA) no sinal CPMG, é realizada média sobre os cálculos do KBDM, resultando na distribuição das possíveis soluções do sistema, ou seja, num histograma da distribuição dos tempos de relaxação $T_{2}$.

Fonte: Elaborada pelo autor.

Para efetiva demonstração da funcionalidade dessa versão do KBDM como método para obtenção de curvas de distribuição $T_{2}$ analisamos uma série de amostras bem conhecidas, e os resultados são comparados com as distribuições obtidas com método mundialmente estabelecido da Inversão de Laplace com regularização de Tikhonov e SVD. ${ }^{89}$ 


\section{Análise exponencial}

O primeiro estudo realizado foi da análise da influência dos diferentes parâmetros experimentais e computacionais na determinação dos histogramas de tempos de relaxação $T_{2}$. Para isso foram utilizadas as medidas realizadas com a cerâmica de alumina, pois é uma cerâmica bem conhecidas e caracterizadas por intrusão de mercúrio e raio-x. Foi analisado o comportamento perante o número de médias experimentais, número de interações do código e parâmetro de regularização.

Iniciamos apresentando uma visualização da lista de parâmetros (line list) calculadas pelo KBDM a partir de um sinal CPMG da cerâmica de alumina. A Fig. 6.9 apresenta a amplitude das componentes $\left|d_{k}\right|$ em função da frequência $f_{k}$. Sabemos que o sinal CPMG é composto por uma somatória de decaimento exponenciais de frequência nula, logo essa visualização da lista de parâmetros obtidas torna evidente as componentes espúrias e as principais do ajuste.

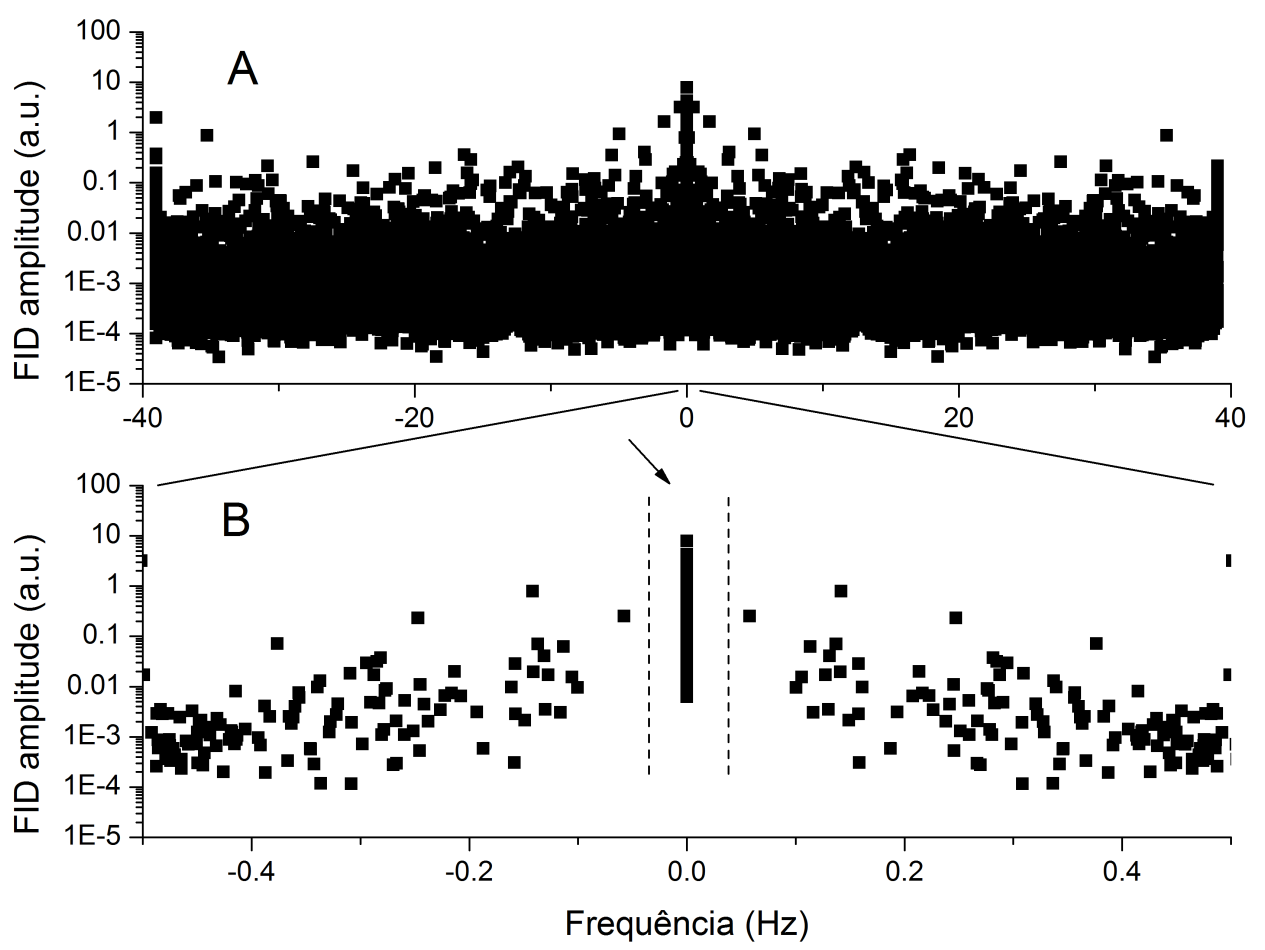

Figura 6.9- Visualização da lista de parâmetros obtida na análise espectral com o KBDM do sinal CPMG da cerâmica de alumina. Em A) temos a visualização completa da faixa de frequência e em B) a região próximo de zero expandida. Uma vez que estamos parametrizando um sinal composto por uma soma de exponenciais com frequência nula, apenas as componentes selecionadas em B) já retornam um ótimo ajuste do sinal experimental. Os demais pontos são gerados no processo de fitting devido o ruído presente no sinal. O processamento KBDM foi realizado com PNA 2\% e 100 médias computacionais.

Fonte: Elaborada pelo autor. 
Apenas os picos na posição de frequência nula representam componentes reais do sinal CPMG, sendo que os demais pontos surgem devido ao ruído experimental. Isso é confirmado com a reconstrução do sinal CPMG a partir apenas das componentes selecionadas, que geram um sinal reconstruído com ajuste excelente ao sinal experimental.

O próximo passo é apresentar a influência do ruído experimental presente no sinal CPMG no processamento pelo KBDM. Para isso, foram adquiridos os sinais com 2, 8, 32 e 128 médias da cerâmica de alumina. A distribuição de $T_{2}$ obtida com o KBDM é apresentada na Fig. 6.10 (esquerda), onde foram realizadas 100 médias computacionais e utilizado PNA de $2 \%$. A direita é apresentada as respectivas distribuições de Laplace obtidas com $\alpha=10$ e 100 pontos.
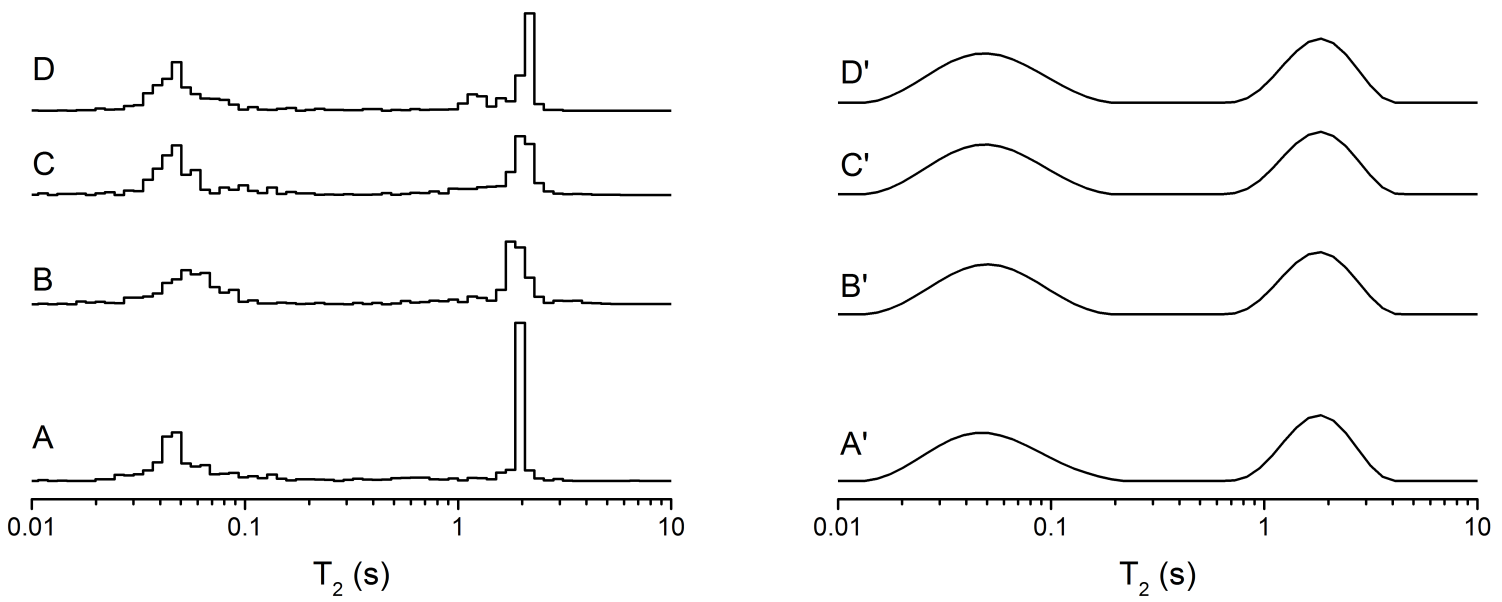

Figura 6.10- Comparação da distribuição de $T_{2}$ através do método do KBDM (esquerda) e do método da Inversão de Laplace (direita) em função do número de médias experimentais do sinal CPMG das cerâmicas de alumina, com A) 2, B) 8, C) 32 e D) 128 médias. As distribuições por KBDM foram calculadas com 100 médias computacionais e PNA de $2 \%$. As distribuições de Laplace foram calculadas com $\alpha=10$ e 100 pontos.

Fonte: Elaborada pelo autor.

Os sinais obtidos com apenas duas médias experimentais foram suficientes na determinação das distribuições e a influência do número de médias apresenta-se como mínima, onde apenas nota-se que tempos de relaxação menores sofrem uma perda de definição conforme menor a relação $s / r$ do sinal.

O número de médias computacionais, ou interações, do processamento com o KBDM também altera o resultado obtido. A Fig. 6.11 apresenta as distribuições obtidas com 4, 16, 64 e 256 médias computacional. Como esperado, aumentando-se o número de médias a distribuição dos picos se torna mais contínua. Diversos testes foram realizados para mostrar 
que na faixa de trabalho de 100 médias, distribuição com resolução satisfatórias são obtidas.

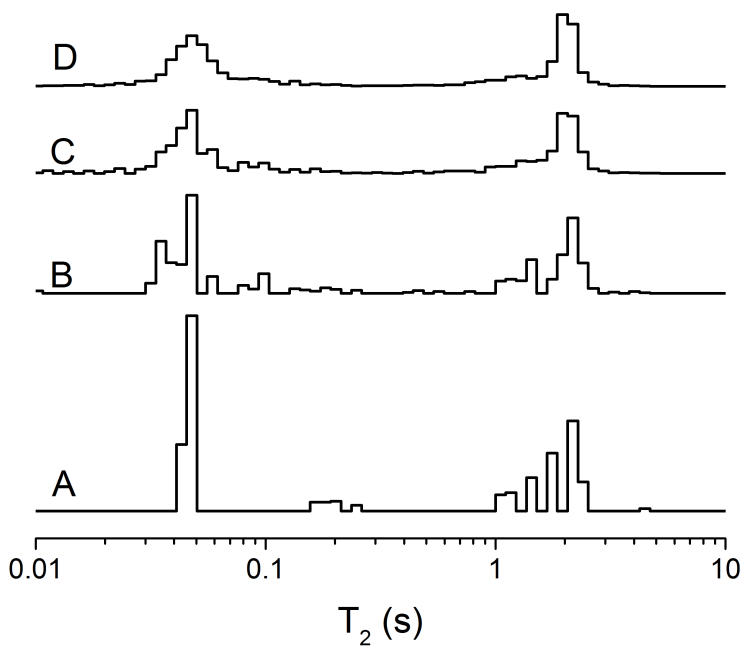

Figura 6.11- Comparação das distribuições $T_{2}$ obtidas pelo KBDM, em função do número de médias computacionais realizada, A) 4, B) 16, C) 64 e D) 256. Todas as distribuições foram calculadas com PNA $=2 \%$ e com o a sinal experimental obtido com 32 scans da cerâmica de alumina.

Fonte: Elaborada pelo autor.

Por fim, a Fig. 6.12 mostra que a influência do valor utilizado na regularização (PNA) na determinação das distribuições apresenta-se com característica semelhante ao parâmetro $\alpha$ da Inversão de Laplace. Nota-se na Fig. 6.12 A) que para PNA nulo, o resultado obtido corresponde a uma distribuição discreta de $T_{2}$, com forte vínculo com o resultado obtido pela Laplace com valores baixo de $\alpha=10^{-3}$. Conforme maior a porcentagem do parâmetro PNA, a distribuição começa a dispersar pelo espectro, assim como observado em análises de Inversão de Laplace com $\alpha$ maiores.

A comparação apresentada na Fig. 6.12 mostra que o comportamento geral dos dois métodos é semelhante, onde parâmetros de regularização baixos resultam em componentes semelhantes, e para parâmetros altos as distribuições tornam-se muito largas. Desse modo, a escolha dos parâmetros de regularização PNA e $\alpha$ devem ser sempre otimizados, porém ainda dependem da intuição física do experimento realizado.

\section{Resolução}

Outro aspecto importante a ser explorado é a resolução dos picos das distribuições. Para estudar essa característica sinais CPMG dos phantons construídos com água dopada com $\mathrm{CuSO}_{4}(\mathrm{~S} 1-\mathrm{S} 12)$ foram adquiridas, colocando as amostras duas a duas no magneto (S1+S12), 

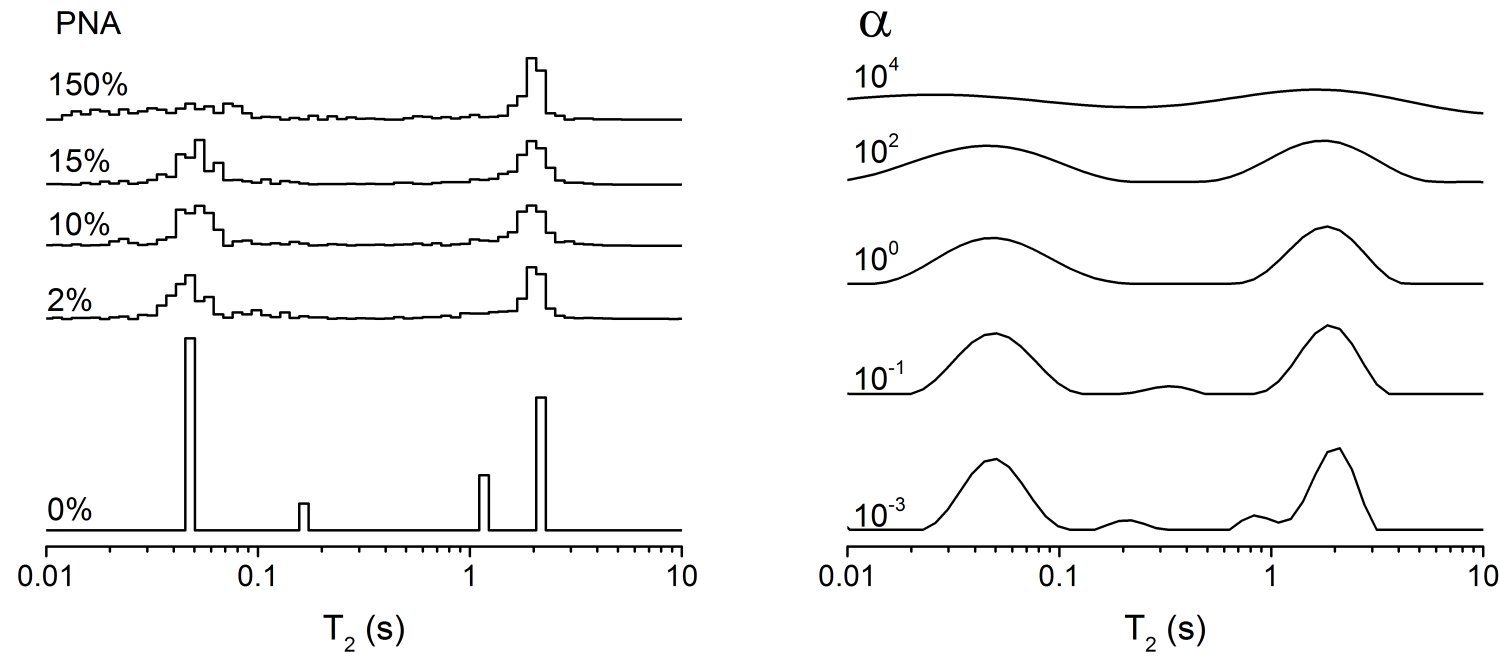

Figura 6.12- Comparação da distribuição de $T_{2}$ pelo método do KBDM (esquerda) e Inversão de Laplace (direita) calculada em função do parâmetro de regularização (PNA) no caso do FDM com 0 , $2 \%, 10 \%, 15 \%$ e E) $150 \%$. Para a Inversão de Laplace foi calculado em função do parâmetro de regularização $\alpha A^{\prime}$ ) 0,001, B') 0,1, C') 1, D') 100 e E') 10.000, todos com 200 pontos.

Fonte: Elaborada pelo autor.

$(\mathrm{S} 2+\mathrm{S} 12)$ até $(\mathrm{S} 11+\mathrm{S} 12)$. A Fig. 6.13 apresenta uma comparação da distribuição obtida com a inversão de Laplace e pelo KBDM.

Na Fig. $6.13 \mathrm{~A}$ ) é apresentada a comparação da distribuição de tempos de relaxação $T_{2}$ obtidos com a Inversa de Laplace (preto) e pelo KBDM (vermelho). Uma vez que os sinais CPMG foram adquiridos com as soluções aquosas em conjunto duas a duas, a primeira curva na parte de baixo foi obtida do conjunto $(\mathrm{S} 1+\mathrm{S} 12)$ com constantes de tempos $T_{2, S 1}=0,015$ s e $T_{2, S 12}=2,42 \mathrm{~s}$, a segunda curva acima é o conjunto $(\mathrm{S} 2+\mathrm{S} 12)$, e assim continuamente, até a curva superior do gráfico formada pelo conjunto $(\mathrm{S} 11+\mathrm{S} 12)$ que apresenta os dois picos muito próximos em $T_{2, S 11}=2,1 \mathrm{~s}$ e $T_{2, S 12}=2,42 \mathrm{~s}$.

Sinais CPMG dessas mesmas soluções foram adquiridas e analisadas separadamente e o fitting mono-exponencial realizado. Os valores de $T_{2}$ obtidos são apresentados na Tabela 6.1.

Tabela 6.1- Tempos de relaxação $T_{2}$ de cada solução.

\begin{tabular}{ccccccccccccc}
\hline Solução & $S_{1}$ & $S_{2}$ & $S_{3}$ & $S_{4}$ & $S_{5}$ & $S_{6}$ & $S_{7}$ & $S_{8}$ & $S_{9}$ & $S_{10}$ & $S_{11}$ & $S_{12}$ \\
\hline$T_{2}(\mathrm{~s})$ & 0,015 & 0,032 & 0,061 & 0,126 & 0,24 & 0,44 & 0,74 & 1,0 & 1,16 & 1,62 & 2,10 & 2,42 \\
\hline
\end{tabular}
Fonte: Elaborada pelo autor.

Note que na Fig. 6.13 A) as distribuições obtidas pelo KBDM apresentam picos mais estreitos, centrados na distribuição obtida pela Inversa de Laplace. Para os três conjuntos com 
picos mais próximos (S9+S12), $(\mathrm{S} 10+\mathrm{S} 12)$ e $(\mathrm{S} 11+\mathrm{S} 12)$, os picos apresentam-se sobrepostos na Inversa de Laplace, onde foi utilizado $\alpha=0,1$. Na Fig. 6.13 B) apresentamos novamente o conjunto $(\mathrm{S} 9+\mathrm{S} 12),(\mathrm{S} 10+\mathrm{S} 12)$ e $(\mathrm{S} 11+\mathrm{S} 12)$, porém agora com a Inversa de Laplace processada com $\alpha$ reduzido $(0,0001)$ necessário para separar os picos (preto). Dependendo do $\alpha$ utilizado e da região escolhida a solução da inversa de Laplace é parcialmente estável, retornando picos dentro da região previamente obtida $\operatorname{com} \alpha$ maior.
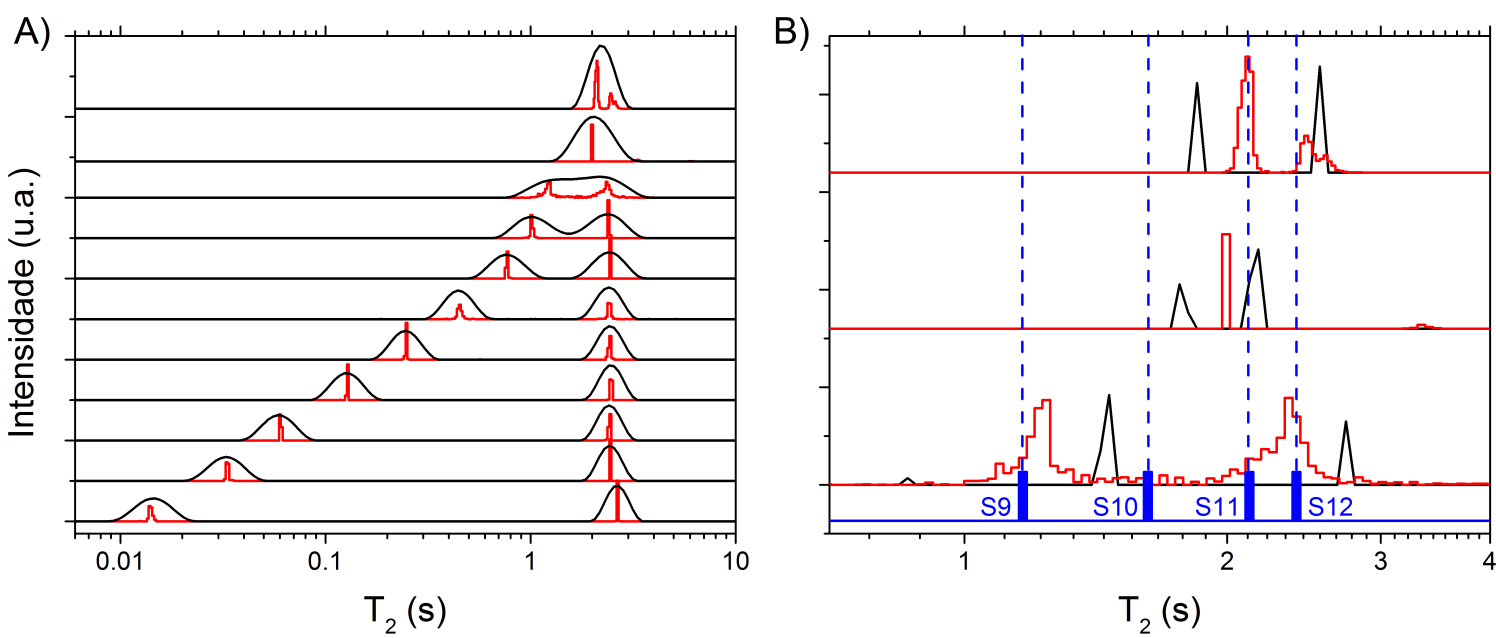

Figura 6.13- A) Comparação da distribuição de $T_{2}$ pelo método do KBDM (vermelho) e Inversão de Laplace (preto) para os sinais adquiridos duas a duas, das soluções aquosas de $C u S O_{4}$, de baixo pra cima $(\mathrm{S} 1+\mathrm{S} 12),(\mathrm{S} 2+\mathrm{S} 12)$ até $(\mathrm{S} 11+\mathrm{S} 12)$. As Inversões da Laplace foram realizadas com $\alpha=$ 0,1 . Em B) é apresentada em azul os valores de $T_{2}$ de cada solução $S_{9}$ até $S_{12}$, comparados com os valores obtidos pelo KBDM (vermelho) e pela Inversão de Laplace (preto) com $\alpha=$ 0,0001 . Com $\alpha$ reduzido é possível a presença de dois picos, porém com pouca precisão.

Fonte: Elaborada pelo autor.

Na Fig. 6.13 B) os valores de fitting mono-exponencial das soluções S9, S10, S11 e S12 são apresentados em cor azul. A comparação do KBDM (vermelho) e da Inversa de Laplace (preto) com os valores esperados (azul) mostra que o KBDM resolveu os picos do conjunto $(\mathrm{S} 9+\mathrm{S} 12)$ e $(\mathrm{S} 11+\mathrm{S} 12)$ com maior precisão, porém no caso $(\mathrm{S} 10+\mathrm{S} 12)$ houve um colapso em um único pico, centrado no valor médio de S10 e S12. A Inversa de Laplace nas três situações determina a presença de duas componentes, porém a posição central dos picos se apresentam altamente instável, mudando de posição de acordo com o $\alpha$ e região da solução estabelecida. A razão para esse comportamento da Laplace e do KBDM em sistemas com picos tão próximos advém da intrínseca limitação das técnicas perante sinais ruidosos, sendo que o sistema não tem resolução suficiente nessa faixa. 


\section{Aplicações}

Nesta seção apresentamos resultados obtidos da distribuição de tempos de relaxação de sinais CPMG adquiridos de grãos, óleos e rochas porosas. O resultado do KBDM é comparado com a Transformada Inversa de Laplace utilizando o método NNLS-ILT (non-negative least squares inverse Laplace transform) usando regularização de Tikhonov e SVD. ${ }^{89}$

A Fig. $6.14 \mathrm{~A}$ ) apresenta a distribuição de $T_{2}$ para um composto de amendoim umedecido com água, obtidos com o KBDM (vermelho) e a Inversa de Laplace com $\alpha=10$ (azul) e $\alpha=0,1$ (preto). Nesse sistema, sabemos que três tempos de relaxação $T_{2}$ são esperados, sendo a componente da água a mais longa, em 2 segundos, e os dois tempos em torno de 0,1 segundos característicos do óleo do amendoim. A Inverda de Laplace com $\alpha$ pequeno resultou na distribuição dos tempos com os dois picos em torno de $0,1 \mathrm{~s}$ melhor definidos, porém introduziu picos espúrios em tempos de relaxação abaixo de $10^{-2} \mathrm{~s}$. O resultado do KBDM foi conciso nesse caso, apresentando-se como uma ferramenta útil no auxilio na determinação das tempos de relaxação.

Em Fig. 6.14 B), apresentamos a distribuição dos tempos de relaxação de óleo lubrificante. Temos que para $\alpha=0,1$ (preto) a distribuição apresenta quatro picos de $T_{2}$, e para $\alpha=1$ (azul) apenas três. A situação de $\alpha=1$ concorda bem com o resultado obtido pelo KBDM. Por se tratar de um problema mal-posto, com mais de uma solução possível, esse resultado mostra que o KBDM pode ser uma ferramenta útil para corroborar uma melhor solução do sistema.

Por fim, na Fig. 6.14 C) apresentamos a comparação dos tempos de relaxação obtidos para as rochas porosas Sillurian, Indiana e Combfield. Os sinais CPMG foram adquiridos em $2 \mathrm{MHz}$ com tempo ao eco de $300 \mu$ s e 32 médias. Dois valores de $\alpha$ são apresentados e os resultados mostram que o KBDM gera distribuição de tempos de relaxação satisfatórios, mesmo em situação complexas como no caso das rochas porosas.

Os resultados apresentados mostram que o método proposto para análise exponencial pelo KBDM é eficiente na determinação da distribuição de tempos de relaxação $T_{2}$ a partir de sinais CPMG. Foram analisadas diversos sistemas, incluindo soluções aquosa com $\mathrm{CuSO}_{4} .5 \mathrm{H}_{2} \mathrm{O}$, sementes, óleos, cerâmicas e rochas porosas Sillurian, Indiana e Combfield. Em todos os casos, 

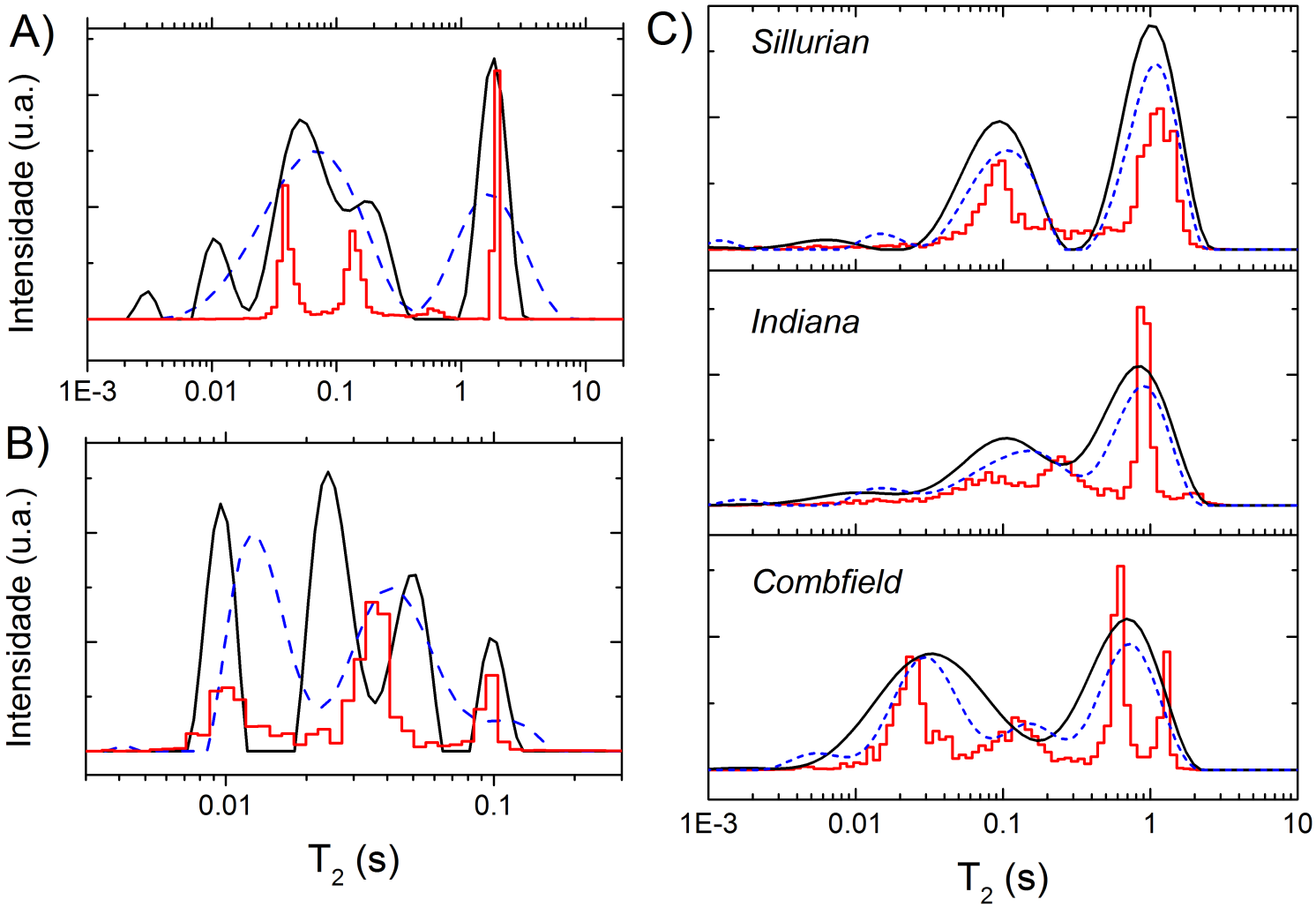

Figura 6.14- Distribuição de tempos $T_{2}$ pela ILT e KBDM para A) amendoim umidecido, B) Óleo lubrificante e C) Rochas porosas Sillurian, Indiana e Combfield. Em vermelho sinais gerados pelo KBDM, azul Laplace com $\alpha$ maior e preto $\alpha$ menor.

Fonte: Elaborada pelo autor.

os resultados mostram que as informações extraídas pelo método do KBDM são compatíveis com os obtidos pela Inversão de Laplace baseada no método mundialmente utilizado NNLS-ILT com regularização de Tikhonov e SVD. 



\section{Capítulo 7}

\section{Conclusão}

A principal conclusão desse trabalho é que as sequências de pulsos tipo Steady State Free Precession com alternância e incremento de fase, tem grande potencial de aplicação em RMN tanto em alta quanto em baixa resolução.

Em alta resolução concluímos que a SSFPdx e SSFPdxdt, respectivamente com incremento linear e quadrático no ciclo de fases, removem as anomalias de fase e amplitude observadas nos espectros SSFP, reduzindo em cerca de uma ordem de grandeza o tempo total de aquisição dos sinais quando comparado com as técnicas convencionais. Os resultados mais significativos foram obtidos com a proposta da SSFPdxdt que além de remover as anomalias espectrais, reduz o tempo total de aquisição da SSFP com múltiplas aquisições, ao não necessitar esperar a formação de novos estados estacionários para promediar os sinais.

Em baixa resolução, concluímos que alternâncias de fase na Continuous Wave Free Precession remove a dependência da sequência com o offset de frequência e com o tempo entre pulsos. A CP-CWFPx-x apresenta a maior região dinâmica e sua implementação com ângulo de refocalização pequeno possibilita a estimativa rápida dos tempos de relaxação longitudinal em uma única aquisição. Concluímos também que a $C W F P-T_{1}$ é robusta e rápida e se apresenta como forte candidata para análise rápida de rotinas no setor industrial em sistemas com forte dependência de $T_{1}$.

No estágio de pesquisa no exterior no grupo do Prof. Bernhard Bluemich, concluímos que as sequências Driven-Equilibrium-CPMG e Split $180^{\circ}$ são técnicas promissoras para utilização em magnetos compactos, resultando na estimativa rápida dos mapas de correlação $T_{1}-T_{2}$ e 
na determinação dos tempos de relaxação.

Por fim, concluímos que o método de processamento de dados Krylov Basis Diagonalization Method é eficiente na análise dos sinais truncados da SSFP, possibilitando a deconvolução da componente eco, remoção dos ruídos na linha de base e aumentando a resolução da Transformada de Fourier em algumas situações. A implementação do KBDM para obtenção da distribuição de tempos de relaxação da CPMG ou CWFP se mostrou robusta e eficiente, servindo como via alternativa a Transformada Inversa de Laplace. 


\section{Capítulo 8}

\section{Perspectivas}

As sequências de pulsos no estado estacionário em alta e baixa resolução se apresentam principalmente como vias alternativas rápidas aos métodos padrões na determinação de espectros de frequências e na determinação dos tempos de relaxação.

Rapidez nas análises é um fator importante em estudos que envolvam processos dinâmicos, tais como monitoramento de reações químicas e processos, como no controle de qualidade ou rotina no setor industrial. Dividimos as perspectivas futuras em duas categorias, baixa e alta resolução.

\section{Baixa resolução}

Em baixa resolução, vale citar as possibilidades das aplicações da CWFP, DECPMG e Split $180^{\circ}$ no setor industrial, através da utilização de magnetos compactos em sistemas magnéticos unilaterais ou com alta homogeneidade de campo.

A CWFP com incremento de fase foi previamente analisada em simulações que sugerem aumento na razão $\mathrm{s} / \mathrm{r}$ em sistemas de baixa homogeneidade de campo magnético. Além disso, a exploração das CWFP em sistemas multi-exponenciais pode resultar em métodos mais rápidos e robustos. Outra possibilidade é desenvolver a CWFP e CWFP- $T_{1}$ bi-dimensional para aquisição de mapas de correlação $T_{1}-T_{2}$.

Sobre os métodos de processamento de dados um desenvolvimento interessante seria o método KBDM-2D na análise de experimentos bi-dimensionais. Através de uma versão do 
KBDM-2D obteríamos uma via alternativa no processamento da Transformada Inversa de Laplace bi-dimensional.

\section{Alta resolução}

Em alta resolução as principais perspectivas do métodos apresentados são o emprego das técnicas na análise de compostos biológicos de baixa sensibilidade. Além disso, o desenvolvimento do método para determinação dos tempos de relaxação dos espectros com uma versão da CWFP em alta resolução, ver apêndice A, teria grande utilidade no estudo de conformação e mobilidade de núcleos de baixa sensibilidade.

Vale citar também a possibilidade do emprego das SSFP com incremento não-linear de fase em MRI. Nossos resultados sugerem que a SSFP com incremento não-linear apresentaria a mesma qualidade de imagem das técnicas com múltiplas aquisições SSFP e possivelmente com a vantagem de redução do tempo de aquisição total. 


\section{Referências}

1 RABI, I.; ZACHARIAS, J.; MILLMAN; KUSCH, P. A new method of measuring nuclear magnetic moment. Physical Review, v. 53, p. 318-318, 1938. doi: 10.1103/PhysRev.53.318.

2 PURCELL, E.; TORREY, H.; POUND, R. Resonance absorption by nuclear magnetic moments in a solid. Physical Review, v. 69, p. 37-38, 1946. doi: 10.1103/PhysRev.69.37.

3 BLOCH, F.; HANSEN, W. W.; PACKARD, M. The nuclear induction experiment. Physical Review, v. 70, p. 474-485, 1946. doi: 10.1103/PhysRev.70.474.

4 BLOCH, F. Nuclear induction. Physical Review, v. 70, p. 460-474, 1946. doi: 10.1103/PhysRev.70.460.

5 ARNOLD, J.; DHARMATTI, S.; PACKARD, M. Chemical effects on nuclear induction signals from organic compounds. Journal of Chemical Physics, v. 19, n. 4, p. 507-507, 1951. doi: $10.1063 / 1.1748264$.

6 BIBLE, R. H. NMR Instrumentation since the Varian A-60. Applied Spectroscopy, v. 24, n. 3, p. 326-332, 1970. doi: 10.1366/000370270774371705.

7 FREEMAN, R. The Fourier transform revolution in NMR spectroscopy. Analytical Chemistry, v. 65, p. 743-753, 1993. doi: 10.1021/ac00065a003.

8 COOLEY, J. W.; TUKEY, J. W. An algorithm for the machine calculation of complex Fourier series. Mathematics of Computation, v. 19, p. 297-301, 1965. doi: 10.1090/S00255718-1965-0178586-1.

9 BUTLER, J. P.; REEDS, J. A.; DAWSON, S. V. Estimating solutions of first kind integral equations with nonnegative constraints and optimal smoothing. Journal on Numerical Analysis, v. 18, p. 381-397, 1981. doi: 10.1137/0718025.

10 PROVENCHER, S. W. CONTIN: a general purpose constrained regularization program for inverting noisy linear algebraic and integral equations. Computer Physics Communications, v. 27, n. 3, p. 229-242, 1982. doi: 10.1016/0010-4655(82)90174-6. 
11 ERNST, R. R.; ANDERSON, W. A. Application of Fourier transform spectroscopy to magnetic resonance. Review of Scientific Instruments, v. 37, n. 1, p. 93-102, 1966. doi: 10.1063/1.1719961.

12 FREEMAN, R.; MORRIS, G. A. The Varian story. Journal of Magnetic Resonance, v. 250, p. $80-84,2015$. doi: 10.1016/j.jmr.2014.12.001.

13 ERNST, R. R. Ernst, richard r.: The success story of fourier transformation in NMR. Encyclopedia of Magnetic Resonance, 2007. doi: 10.1002/9780470034590.emrhp0051.

14 ABRAGAM, A. The principles of nuclear magnetism. Clarendon: Oxford University Press, 1961.

15 LEVITT, M. H. Spin dynamics basis of nuclear magnetic resonance. Chichester: John Wiley \& Sons, Ltd, 2008.

16 JACOBSEN, N. E. NMR spectroscopy explained. New Jersey: John Wiley \& Sons, Ltd, 2007.

17 CALLAGHAN, P. T. Principles of nuclear magnetic resonance microscopy. Oxford: Clarendon Press, 1991.

18 KEELER, J. Understanding NMR spectroscopy. Cambridge: John Wiley \& Sons, Ltd, 2002.

19 HAHN, E. L. Spin Echoes. Physical Review, v. 80, p. 580-594, 1950. doi: 10.1103/PhysRev.80.580.

20 CARR, H. Y.; PURCELL, E. M. Effects of diffusion on free precession in nuclear magnetic resonance experiments. Physical Review, v. 94, 1954. doi: 10.1103/PhysRev.94.630.

21 MEIBOOM, S.; GILL, D. Modified spin-echo method for measuring nuclear relaxation times. Review of Scientific Instruments, v. 29, 1958. doi: 10.1063/1.1716296.

$22 \mathrm{KIM}, \mathrm{M}$.; LEE, S. Spin echoes after arbitrary N pulses. Journal of Magnetic Resonance, v. 125, n. 1 , p. $114-119$, 1997. doi: 10.1006/jmre.1996.1077.

23 HENNIG, J. Echoes - how to generate, recognize, use or avoid them in mr-imaging sequences. part I: fundamental and not so fundamental properties of spin echoes. Concepts in Magnetic Resonance, v. 3, n. 3, p. 125-143, 1991. doi: 10.1002/cmr.1820030302.

24 HENNIG, J. Echoes - how to generate, recognize, use or avoid them in MR-imaging sequences. part II: echoes in imaging sequences. Concepts in Magnetic Resonance, v. 3, n. 4, p. 179-192, 1991. doi: 10.1002/cmr.1820030402. 
25 BRADFORD, R.; CLAY, C.; STRICK, E. A steady-state transient technique in nuclear induction. Physical Review, v. 84, p. 157-158, 1951. doi: 10.1103/PhysRev.84.157.

26 CARR, H. Y. Steady-state free precession in nuclear magnetic resonance. Physical Review, v. 112, p. 1693-1701, 1958. doi: 10.1103/PhysRev.112.1693.

27 FREEMAN, R.; HILL, H. Phase and intensity anomalies in fourier transform NMR. Journal of Magnetic Resonance, v. 4, n. 3, p. 366 - 383, 1971. doi: 10.1016/0022-2364(71)90047-3.

28 SCHWENK, A. NMR pulse technique with high sensitivity for slowly relaxing systems. Journal of Magnetic Resonance, v. 5, n. 3, p. 376 - 389, 1971. doi: 10.1016/00222364(71)90088-6.

29 SCHWENK, A. Steady-state techniques for low sensitivity and slowly relaxing nuclei. Encyclopedia of Magnetic Resonance, v. 8, n. 1, p. 4719-4727, 2007. doi: 10.1002/9780470034590.emrstm0531.

30 BECKER, E. D.; FERETTI, J. A.; FARRAR, T. C. Driven equilibrium fourier transform spectroscopy: A new method for nuclear magnetic resonance signal enhancement. Journal of the American Chemical Society, v. 91, n. 27, p. 7784-7785, 1969. doi: 10.1021/ja50001a068.

31 FREEMAN, R.; MORRIS, G. A. The 'DANTE' experiment. Journal of Magnetic Resonance, v. 213, n. 2, p. $244-246$, 2011. doi: 10.1016/j.jmr.2011.08.020.

32 PATZ, S. Some factors that influence the steady state in steady-state free precession. Magnetic Resonance Imaging, v. 6, n. 4, p. 405 - 413, 1988. doi: 10.1016/0730-725X(88)90477-8.

33 HARGREAVES, B. Rapid gradient-echo imaging. Journal of Magnetic Resonance Imaging, v. 36, n. 6, p. 1300-1313, 2012. doi: 10.1002/jmri.23742.

34 MONARETTO, T.; ANDRADE, F. D.; MORAES, T. B.; SOUZA, A. A.; DEAZEVEDO, E.; COLNAGO, L. On resonance phase alternated CWFP sequences for rapid and simultaneous measurement of relaxation times. Journal of Magnetic Resonance, v. 259, p. $174-178,2015$. doi: $10.1016 /$ j.jmr.2015.08.013.

35 MITCHELL, J.; HURLIMANN, M.; FORDHAM, E. A rapid measurement of $T_{1} / T_{2}$ : the DECPMG sequence. Journal of Magnetic Resonance, v. 200, n. 2, p. 198 - 206, 2009. doi: 10.1016/j.jmr.2009.07.002.

36 FREED, D.; SCHEVEN, U.; HURLIMANN, M. Split $180^{\circ}$ sequences. Diffusion-Fundamentals: basic principles of diffusion theory, experiment and application, v. 10, p. 19.1-19.3, 2009. Disponível em: <https://www.unileipzig.de/diff/pdf/volume10/difffund102820092919.pdf>. Acesso em 18 mar. 2016. 
37 AZEREDO, R.; COLNAGO, L.; ENGELSBERG, M. Quantitative analysis using steadystate free precession nuclear magnetic resonance. Analytical Chemistry, v. 72, n. 11, p. 2401-2405, 2000. doi: 10.1021/ac991258e.

38 AZEREDO, R.; COLNAGO, L.; SOUZA, A.; ENGELSBERG, M. Continuous wave free precession: practical analytical tool for low-resolution nuclear magnetic resonance measurements. Analytica Chimica Acta, v. 478, n. 2, p. 313 - 320, 2003. doi: 10.1016/S0003-2670(02)015143.

39 VENÂNCIO, T.; ENGELSBERG, M.; AZEREDO, R. B.; ALEM, N. E.; COLNAGO, L. A. Fast and simultaneous measurement of longitudinal and transverse NMR relaxation times in a single continuous wave free precession experiment. Journal of Magnetic Resonance, v. 173, n. 1, p. $34-39$, 2005. doi: 10.1016/j.jmr.2004.11.016.

40 KRONENBITTER, J.; SCHWENK, A. A new technique for measuring the relaxation times $T_{1}$ and $T_{2}$ and the equilibrium magnetization $M_{0}$ of slowly relaxing systems with weak NMR signals. Journal of Magnetic Resonance, v. 25, n. 1, p. 147 - 165, 1977. doi: 10.1016/00222364(77)90127-5.

41 VEnÂNCIO, T.; ENGElSBERG, M.; AZEREDO, R. B.; COlnAGO, L. A. Thermal diffusivity and nuclear spin relaxation: a continuous wave free precession NMR study. Journal of Magnetic Resonance, v. 181, n. 1, p. 29 - 34, 2006. doi: 10.1016/j.jmr.2006.03.011.

42 CAROSIO, M. G.; BeRnARDES, D. F.; ANDRADE, F. D.; MORAES, T. B.; TOSIN, G.; COLNAGO, L. A. Measuring thermal properties of oilseeds using time domain nuclear magnetic resonance spectroscopy. Journal of Food Engineering, v. 173, p. 143 - 149, 2016. doi: 10.1016/j.jfoodeng.2015.11.004.

43 CORRÊA, C. C.; FORATO, L. A.; COLNAGO, L. A. High-throughput non-destructive nuclear magnetic resonance method to measure intramuscular fat content in beef. Analytical and Bioanalytical Chemistry, v. 393, n. 4, p. 1357-1360, 2008. doi: 10.1007/s00216-0082526-6.

44 DE ANDRADE, F. D.; NETTO, A. M.; COLNAGO, L. A. Qualitative analysis by online nuclear magnetic resonance using Carr-Purcell-Meiboom-Gill sequence with low refocusing flip angles. Talanta, v. 84, n. 1, p. 84 - 88, 2011. doi: 10.1016/j.talanta.2010.12.033.

45 COLNAGO, L. A.; ANDRADE, F. D.; SOUZA, A. A.; AZEREDO, R. B. V.; LIMA, A. A.; CERIONI, L. M.; OSAN, T. M.; PUSIOL, D. J. Why is inline NMR rarely used as industrial sensor? challenges and opportunities. Chemical Engineering and Technology, v. 37, n. 2, p. 191-203, 2014. doi: 10.1002/ceat.201300380.

46 COlnAGO, L. A.; AZEREDO, R. B. V.; NeTTO, A.; ANDRADE, F. D.; VENÂNCIO, T. Rapid analyses of oil and fat content in agri-food products using continuous wave free precession time domain NMR. Magnetic Resonance in Chemistry, v. 49, p. S113-S120, 2011. doi: $10.1002 / \mathrm{mrc} .2841$. 
47 DE ANDRADE, F. D.; NETTO, A. M.; COLNAGO, L. A. Use of Carr-Purcell pulse sequence with low refocusing flip angle to measure $T_{1}$ and $T_{2}$ in a single experiment. Journal of Magnetic Resonance, v. 214, p. 184 - 188, 2012. doi: 10.1016/j.jmr.2011.11.004.

48 SANTOS, P. M. D.; SOUZA, A. A. D.; COLNAGO, L. A. Supressão das anomalias de fase e batimentos laterais em espectros de RMN ${ }^{13} \mathrm{C}$ obtidos com a sequências de precessão livre no estado estacionário. Química Nova, v. 33, p. 954 - 956, 2010. doi: 10.1590/S010040422010000400034.

49 SANTOS, P. M.; SOUZA, A. A.; COLNAGO, L. A. Fast acquisition of ${ }^{13} C$ NMR spectra using the steady-state free precession sequence. Applied Magnetic Resonance, v. 40, n. 3, p. 331-338, 2011. doi: 10.1007/s00723-011-0209-5.

50 NUNES, L. M.; MORAES, T. B.; BARBOSA, L. L.; MAZO, L. H.; COLNAGO, L. A. Monitoring electrochemical reactions in situ using steady-state free precession ${ }^{13} C$ NMR spectroscopy. Analytica Chimica Acta, v. 850, p. 1 - 5, 2014. doi: 10.1016/j.aca.2014.05.022.

51 MORAES, T. B.; SANTOS, P. M.; MAGON, C. J.; COLNAGO, L. A. Suppression of spectral anomalies in SSFP-NMR signal by the Krylov Basis Diagonalization Method. Journal of Magnetic Resonance, v. 243, p. 74 - 80, 2014. doi: 10.1016/j.jmr.2014.03.009.

52 DUARTE, C. J.; COLNAGO, L. A.; AZEREDO, R. B. V.; VENÂNCIO, T. Solvent suppression in high-resolution ${ }^{1} H$ NMR spectroscopy using conventional and phase alternated continuous wave free precession. Applied Magnetic Resonance, v. 44, n. 11, p. 1265-1280, 2013. doi: 10.1007/s00723-013-0482-6.

53 DUARTE, C. Precessão livre em onda contínua (CWFP) como ferramenta para supressão do sinal de solvente em RMN de alta resolução. 2011. 84 p. Dissertação (Mestrado em Química Orgânica) - Departamento de Química, Universidade Federal de São Carlos, São Carlos, 2011.

54 RUDAKOV, T. N.; MIKHALTSEVICH, V. T.; SELCHIKHIN, O. P. The use of multi pulse nuclear quadrupole resonance techniques for the detection of explosives containing RDX. Journal of Physics D Applied Physics, v. 30, n. 9, p. 1377, 1997. doi: 10.1088/0022$3727 / 30 / 9 / 013$.

55 BANGERTER, N. K.; HARGREAVES, B. A.; VASANAWALA, S. S.; PAULY, J. M.; GOLD, G. E.; NISHIMURA, D. G. Analysis of multiple-acquisition SSFP. Magnetic Resonance in Medicine, v. 51, n. 5, p. 1038-1047, 2004. doi: 10.1002/mrm.20052.

56 NAYAK, K. S.; LEE, H.-L.; HARGREAVES, B. A.; HU, B. S. Wideband SSFP: alternating repetition time balanced steady state free precession with increased band spacing. Magnetic Resonance in Medicine, v. 58, n. 5, p. 931-938, 2007. doi: 10.1002/mrm.21296.

57 WEBB, A. Increasing the sensitivity of magnetic resonance spectroscopy and imaging. Analytical Chemistry, v. 84, n. 1, p. 9-16, 2012. doi: 10.1021/ac201500v. 
58 ZALESSKIY, S. S.; DANIELI, E.; BLUEMICH, B.; ANANIKOV, V. P. Miniaturization of NMR systems: desktop spectrometers, microcoil spectroscopy, and "NMR on a chip" for chemistry, biochemistry, and industry. Chemical Reviews, v. 114, n. 11, p. 5641-5694, 2014. doi: $10.1021 / \mathrm{cr} 400063 \mathrm{~g}$.

59 RUDAKOV, T. Modification of SSFP technique for the effective detection of NQR signals. Physics Letters A, v. 358, n. 4, p. 322 - 326, 2006. doi: 10.1016/j.physleta.2006.05.024.

60 FREED, D.; HURLIMANN, M.; SCHEVEN, U. The equivalence between off-resonance and on-resonance pulse sequences and its application to steady-state free precession with diffusion in inhomogeneous fields. Journal of Magnetic Resonance, v. 162, n. 2, p. 328 - 335, 2003. doi: 10.1016/S1090-7807(03)00041-7.

61 CASANOVA, F.; PERLO, J.; BLUEMICH, B. Single sided NMR. Berlin: Springer, 2010.

62 PERLO, J. F. Single-sided NMR tomography. 2006. 155 p. Thesis (Doktors der Naturwissenschaften) - Institut für Technische und Makromolekulare Chemie - RWTH Aachen University, Aachen, Germany, 2006.

63 HURLIMANN, M.; GRIFFIN, D. Spin dynamics of carr-purcell-meiboom-gill-like sequences in grossly inhomogeneous $B_{0}$ and $B_{1}$ fields and application to NMR well logging. Journal of Magnetic Resonance, v. 143, n. 1, p. 120 - 135, 2000. doi: 10.1006/jmre.1999.1967.

64 HURLIMANN, M. D. Diffusion and relaxation effects in general stray field NMR experiments. Journal of Magnetic Resonance, v. 148, n. 2, p. 367-378, 2001. doi: 10.1006/jmre.2000.2263.

65 KOROLEVA, V. D.; MANDAL, S.; SONG, Y. Q.; HURLIMANN, M. D. Broadband CPMG sequence with short composite refocusing pulses. Journal of Magnetic Resonance, v. 230, p. 64 - 75, 2013. doi: 10.1016/j.jmr.2013.01.006.

66 MORAES, T. B.; COLNAGO, L. A. Simulação de sinais de RMN através das equações de bloch. Química Nova, v. 37, p. 1410 - 1416, 2014. doi: 10.5935/0100-4042.20140210.

67 RUDAKOV, T. Some aspects of spin-locking effect in nitrogen-14 quadrupolar spinsystem. Chemical Physics Letters, v. 443, n. 4-6, p. 269 - 273, 2007. doi: 10.1016/j.cplett.2007.06.094.

68 RUDAKOV, T.; HAYES, P.; FLEXMAN, J. Optimised NQR pulse technique for the effective detection of heroin base. Solid State Nuclear Magnetic Resonance, v. 33, n. 3, p. 31 - 35, 2008. doi: 10.1016/j.ssnmr.2008.02.001.

69 RUDAKOV, T. N. Some aspects of the effective detection of ammonium nitrate-based explosives by pulsed nqr method. Applied Magnetic Resonance, v. 43, n. 4, p. 557-566, 2012. doi: $10.1007 /$ s00723-012-0330-0. 
70 DEONI, S. C.; WARD, H. A.; PETERS, T. M.; RUTT, B. K. Rapid $T_{2}$ estimation with phase-cycled variable nutation steady-state free precession. Magnetic Resonance in Medicine, v. 52, n. 2, p. 435-439, 2004. doi: 10.1002/mrm.20159.

71 BALTISBERGER, J. H.; WALDER, B. J.; KEELER, E. G.; KASEMAN, D. C.; SANDERS, K. J.; GRANDINETTI, P. J. Communication: phase incremented echo train acquisition in NMR spectroscopy. Journal of Chemical Physics, v. 136, n. 21, 2012. doi: 10.1063/1.4728105.

72 WALDER, B. J.; DEY, K. K.; KASEMAN, D. C.; BALTISBERGER, J. H.; GRANDINETTI, $P$. J. Sideband separation experiments in NMR with phase incremented echo train acquisition. Journal of Chemical Physics, v. 138, n. 17, 2013. doi: 10.1063/1.4803142.

73 SHKARIN, P.; SPENCER, R. G. S. Direct simulation of spin echoes by summation of isochromats. Concepts in Magnetic Resonance, v. 8, n. 4, p. 253-268, 1996. doi: 10.1002/(SICI)1099-0534(1996)8:4<253::AID-CMR2>3.0.CO;2-Y.

74 ZHANG, S.; GORENSTEIN, D. Theory of frequency shifted excitation by phase incremented pulses in nuclear magnetic resonance. Journal of Chemical Physics, v. 105, n. 14, p. 5659-5664, 1996. doi: 10.1063/1.472411.

75 ZHANG, S. Phase incremented pulses in NMR with applications. Annual Reports on NMR Spectroscopy, v. 53, p. 1 - 66, 2004. doi: 10.1016/S0066-4103(04)53001-6.

76 FREED, D. E.; SCHEVEN, U. M.; ZIELINSKI, L. J.; SEN, P. N.; HURLIMANN, M. D. Steady-state free precession experiments and exact treatment of diffusion in a uniform gradient. Journal of Chemical Physics, v. 115, n. 9, p. 4249-4258, 2001. doi: 10.1063/1.1389859.

77 HURLIMANN, M. D. Carr-purcell sequences with composite pulses. Journal of Magnetic Resonance, v. 152, p. 109-123, 2001. doi: 10.1006/jmre.2001.2370.

78 SCHLUMBERGER-TECHNOLOGY-CORPORATION. Denise Freed; Martin D. Hürlimann; Ulrich Scheven. Nuclear magnetic resonance logging based on steady state free precession. US6580272 B2, 6 Nov. 2001, 17 June 2003.

79 SCHWENK, A. Steady-state techniques for low sensitivity and slowly relaxing nuclei. Progress in Nuclear Magnetic Resonance Spectroscopy, v. 17, p. 69 - 140, 1985. doi: 10.1002/9780470034590.emrstm0531.

80 FREED, D. E. The universality of physics: From magnetic flux and incommensurability to NMR and oil wells. Princeton: Plenum Publishers, 2001.

81 HURLIMANN, M. Efficient measurement of $T_{1} / T_{2}$ ratio in porous media. Talk presented at 18th ISMAR Meeting, Rio de Janeiro, 2013. 
82 VENKATARAMANAN, L.; SONG, Y. Q.; HURLIMANN, M. D. Solving fredholm integrals of the first kind with tensor product structure in 2 and 2.5 dimensions. Signal Processing, IEEE Transactions on, v. 50, n. 5, p. 1017-1026, 2002. doi: 10.1109/78.995059.

83 SONG, Y.; VENKATARAMANAN, L.; HURLIMANN, M.; FLAUM, M.; FRULLA, P.; STRALEY, C. $T_{1}-T_{2}$ correlation spectra obtained using a fast two-dimensional laplace inversion. Journal of Magnetic Resonance, v. 154, n. 2, p. 261 - 268, 2002. doi: 10.1006/jmre.2001.2474.

84 SONG, Y. Magnetic resonance of porous media (MRPM): a perspective. Journal of Magnetic Resonance, v. 229, p. 12 - 24, 2013. doi: 10.1016/j.jmr.2012.11.010. Frontiers of In Vivo and Materials MRI Research.

85 FREED, D.; HURLIMANN, M.; SCHEVEN, U.; TERNEAUD, O.; VENKATARAMANAN, L. Nuclear magnetic resonance methods for extracting information about a fluid in a rock. WO2002008789 A3, 20 July 2001, 18 Apr. 2002.

86 DENG, F.; XIAO, L.; LIAO, G.; ZONG, F.; CHEN, W. A new approach of two-dimensional the NMR relaxation measurement in flowing fluid. Applied Magnetic Resonance, v. 45, n. 2, p. 179-192, 2014. doi: 10.1007/s00723-014-0513-y.

87 DENG, F.; XIAO, L.; CHEN, W.; LIU, H.; LIAO, G.; WANG, M.; XIE, Q. Rapid determination of fluid viscosity using low-field two-dimensional NMR. Journal of Magnetic Resonance, v. 247 , p. $1-8,2014$. doi: $10.1016 /$ j.jmr.2014.08.003.

88 MONTRAZI, E. T. Estudo de cerâmicas porosas de alumina através da medida de tempos de relaxação via ressonância magnética nuclear. 2012. 164 p. Dissertação (Mestrado em Física) - Instituto de Física de São Carlos, Universidade de São Paulo, São Carlos, 2012.

89 BORGIA, G.; BROWN, R.; FANTAZZINI, P. Uniform-penalty inversion of multiexponential decay data. Journal of Magnetic Resonance, v. 132, n. 1, p. 65 - 77, 1998. doi: 10.1006/jmre.1998.1387.

90 MORAES, T. B. O método da diagonalização filtrada e suas aplicações para a ressonância magnética. 2011. 100 p. Dissertação (Mestrado em Física) - Instituto de Física de São Carlos, Universidade de São Paulo, São Carlos, 2011.

91 TIKHONOV, A. N. On the regularization of ill-posed problems (russian). Soviet Math Dokl CCCP, Doklady Akademii Nauk SSSR, v. 4, n. 1, p. 49-52, 1963. doi: MR0162378(285577).

92 MORAeS, T. B.; MONARETtO, T.; COlnAGO, L. A. Rapid and simple determination of $T_{1}$ relaxation times in time-domain NMR by continuous wave free precession sequence. Journal of Magnetic Resonance, v. 270, p. 1 - 6, 2016. doi: http://dx.doi.org/10.1016/j.jmr.2016.06.019. 
93 MONARETTO, T. Desenvolvimento e aplicações de sequências de pulsos CWFP com alternância de fase em rmn no domínio do tempo. 2015. 77 p. Dissertação (Mestrado em Físico-Química) - Instituto de Química de São Carlos, Universidade de São Paulo, São Carlos, 2015.

94 SOUZA, A. Estudos de propriedades petrofísicas de rochas sedimentares por ressonância magnetica nuclear. 2012. 236 p. Tese (Doutorado em Ciências e Engenharia de Materiais) Instituto de Física de São Carlos, Universidade de São Paulo, São Carlos, 2012.

95 LOOK, D. C.; LOCKER, D. R. Time saving in measurement of NMR and EPR relaxation times. Review of Scientific Instruments, v. 41, n. 2, p. 250-251, 1970. doi: 10.1063/1.1684482.

96 KAPTEIN, R.; DIJKSTRA, K.; TARR, C. A single-scan fourier transform method for measuring spin-lattice relaxation times. Journal of Magnetic Resonance, v. 24, n. 2, p. 295 300, 1976. doi: 10.1016/0022-2364(76)90039-1.

97 KINGSLEY, P. B. Methods of measuring spin-lattice $\left(T_{1}\right)$ relaxation times: an annotated bibliography. Concepts in Magnetic Resonance, v. 11, n. 4, p. 243-276, 1999. doi: 10.1002/(SICI)1099-0534(1999)11:4<243::AID-CMR5>3.0.CO;2-C.

98 MOREIRA, L.; FERRARI, A.; REIS, R.; MORAES, T.; COLNAGO, L.A. PEREIRA, F. Prediction of beef color using time-domain nuclear magnetic resonance relaxometry data and multivariate analyses. Analytical and Bioanalytical Chemistry, 2016. Submitted.

99 DANIELI, E.; PERLO, J.; BLUEMICH, B.; CASANOVA, F. Small magnets for portable NMR spectrometers. Angewandte Chemie International Edition, v. 49, n. 24, p. 4133-4135, 2010. doi: 10.1002/anie.201000221.

100 DANIELI, E.; PERLO, J.; BLUEMICH, B.; CASANOVA, F. Highly stable and finely tuned magnetic fields generated by permanent magnet assemblies. Physical Review Letters, v. 110 , p. 180801,2013 . doi: 10.1103/PhysRevLett.110.180801.

101 DANIELI, E.; PERLO, J.; DUCHATEAU, A. L. L.; VERZIJL, G. K. M.; LITVINOV, V. M.; BLUEMICH, B.; CASANOVA, F. On-line monitoring of chemical reactions by using bench-top nuclear magnetic resonance spectroscopy. ChemPhysChem, v. 15, n. 14, p. 3060-3066, 2014. doi: 10.1002/cphc.201402049.

102 PERLO, J.; CASANOVA, F.; BLUEMICH, B. Profiles with microscopic resolution by single-sided NMR. Journal of Magnetic Resonance, v. 176, n. 1, p. 64 - 70, 2005. doi: 10.1016/j.jmr.2005.05.017.

103 PERLO, J.; CASANOVA, F.; BLUEMICH, B. Single-sided sensor for high-resolution NMR spectroscopy. Journal of Magnetic Resonance, v. 180, n. 2, p. $274-279$, 2006. doi: 10.1016/j.jmr.2006.03.004. 
104 CHEN, J. Nonlinear methods for high resolution spectral analysis and their applications in nuclear magnetic resonance experiments. 2002. $241 \mathrm{p}$. Thesis (Doctor of Philosophy in Chemistry) - University of California, Irvine, 2002.

105 JIRU, F. Introduction to post-processing techniques. European Journal of Radiology, v. 67, n. 2, p. $202-217$, 2008. doi: 10.1016/j.ejrad.2008.03.005. Clinical 1H MR Spectroscopy.

106 ISTRATOV, A. A.; VYVENKO, O. F. Exponential analysis in physical phenomena. Review of Scientific Instruments, v. 70, n. 2, p. 1233-1257, 1999. doi: 10.1063/1.1149581.

107 SONG, Y. Q. Resolution and uncertainty of laplace inversion spectrum. Magnetic Resonance Imaging, v. 25, n. 4, p. 445 - 448, 2007. doi: 10.1016/j.mri.2006.11.023.

108 WALL, M. R.; NEUHAUSER, D. Extraction, through filterdiagonalization, of general quantum eigenvalues or classical normal mode frequencies from a small number of residues or a short-time segment of a signal. I. theory and application to a quantum-dynamics model. Journal of Chemical Physics, v. 102, n. 20, p. 8011-8022, 1995. doi: 10.1063/1.468999.

109 MANDELSHTAM, V. A. Harmonic inversion of time cross-correlation functions: The optimal way to perform quantum or semiclassical dynamics calculations. Journal of Chemical Physics, v. 108, n. 24, p. 9999-10007, 1998. doi: 10.1063/1.476498.

110 MANDELSHTAM, V. A. FDM: the filter diagonalization method for data processing in NMR experiments. Progress in Nuclear Magnetic Resonance Spectroscopy, v. 38, n. 2, p. 159-196, 2001. doi: 10.1016/s0079-6565(00)00032-7. 


\section{Apêndices}

\section{Apêndice A: SSFP para determinação dos tempos de relaxação}

Testes iniciais foram realizados para a ideia de obter os tempos de relaxação em espectros de alta resolução utilizando uma sequência análoga a CWFP empregada em baixa resolução, aqui denominada CWFP-HR (high resolution).

Vimos na seção 2.3 que $T^{*}$ é o tempo característico da estabilização do estado estacionário, descrito pela equação 2.3.17. A técnica em alta resolução seria baseada na determinação dos parâmetros $T^{*},\left|M_{s s}\right|$ e $M_{0}$ de cada pico espectral, e logo obter os tempos de relaxação $T_{1}$ e $T_{2}$ através das equações

$$
T_{1}=\frac{T^{*} / 2}{\left|M_{s s}\right| / M_{0}} \quad ; \quad T_{2}=\frac{T^{*} / 2}{1-\left|M_{s s}\right| / M_{0}}
$$

Para executar tal proposta, implementamos uma sequência de pulsos no Espectrômetro Bruker AVANCE III, com aquisição continua após a aplicação de cada pulso, emparelhando os FID um a frente do outro, semelhante ao apresentando na Fig. A.1. Realizando a Transformada de Fourier de cada um desses sinais, podemos acompanhar a evolução de cada pico espectral ao estado estacionário.

A Fig. A.1 apresenta um esquema para um espectro com dois picos, onde vemos o decaimentos dos picos até estabilização do estado estacionário. Em Fig. A.1 B) apresentamos a Transforma de Fourier de cada período de aquisição, através do qual podemos obter os parâmetros $T_{\{1,2\}}^{*},\left|M_{s s\{1,2\}}\right|$ e $M_{0\{1,2\}}$ de cada pico do espectro. 


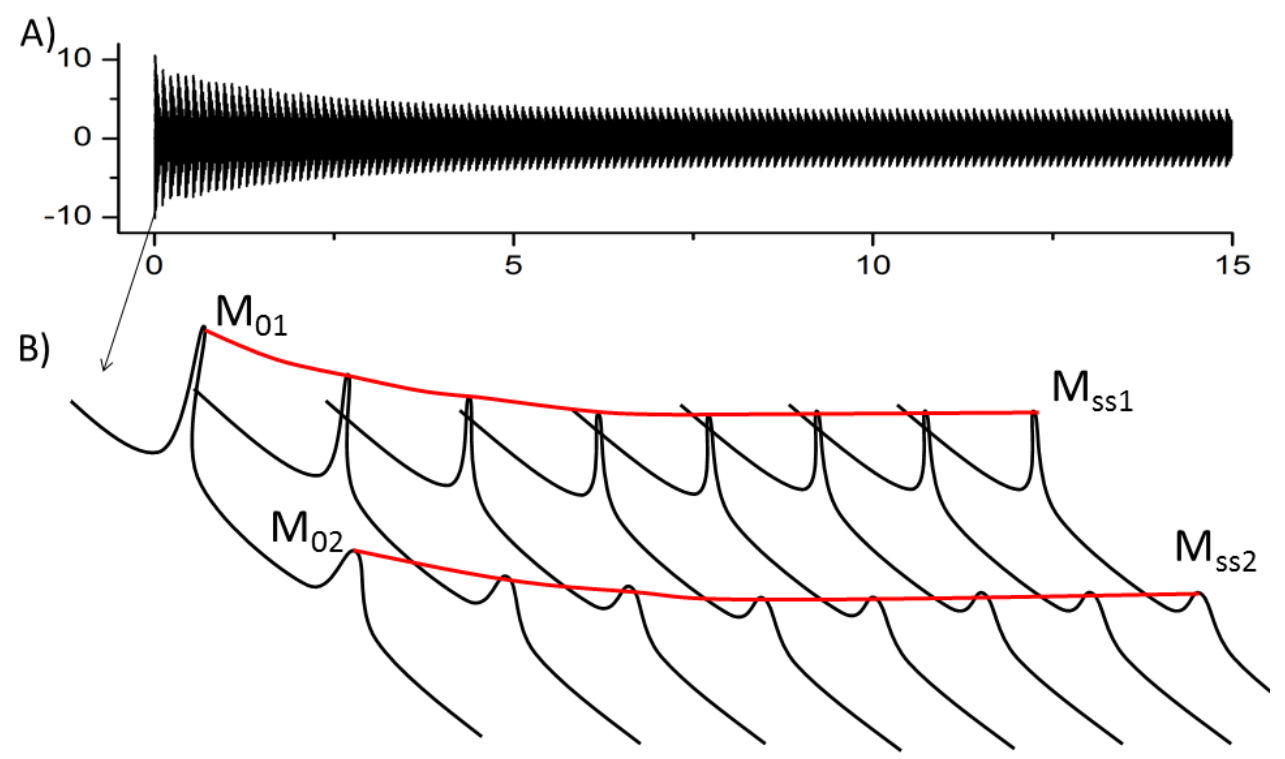

Figura A.1- Esquema da SSFP em alta resolução para determinação rápida dos tempos de relaxação $T_{1}$ e $T_{2}$. Em A) temos a aquisição de todo o comportamento da magnetização até a estabilização do estado estacionário. B) apresenta as Trans. de Fourier de cada período da aquisição, onde através dos tempos de decaimento dos picos e das amplitudes $M_{0}$ e $M_{s s}$ é possível se determinar os tempos de relaxação.

Fonte: Elaborada pelo autor.

Vale resaltar que apenas os picos em posição de frequência $\Omega T_{p}=(2 n+1) \pi$ irão decair ao estado estacionário de acordo com a equação 2.3.17 na CWFP. Isso se resolve utilizando a CWFP com alternância de fase $\pi$ e centralizando o pico em estudo na frequência de offset.

Assim, como primeiro estudo, realizamos experimentos de ${ }^{1} H$ utilizando a CWFP com alternância de fase para uma solução de $20 \mu \mathrm{l}$ de $\mathrm{H}_{2} \mathrm{O}$ e $20 \mu \mathrm{l}$ de acetona em $500 \mu \mathrm{l}$ de $D_{2} O$. Iniciamos realizando a determinação dos tempos de relaxação do sistema $T_{1}$ e $T_{2}$ através das técnicas padrões CPMG (cpmg) e Inversão Recuperação (t1ir). Fig. A.2 apresenta os decaimentos obtidos com a sequência Inversão recuperação a CPMG.

A aquisição CWFP-HR é apresentada na Fig. A.3, mostrando os decaimentos dos picos da $\mathrm{H}_{2} \mathrm{O}$ e da acetona até a estabilização no estado estacionário, com $T_{p}=100 \mathrm{~ms}, 256$ pulsos e 1 média. Na parte inicial dos decaimentos se observa o período de oscilação observados na CWFP, e logo o estado quasi-estacionário, que decai ao estado estacionário, mantendo as amplitudes $M_{0\{1,2\}}$ enquanto a sequência de pulsos continuar sendo aplicada.

A Tabela A.1 apresenta os valores dos tempos de relaxação obtidos com as técnicas CPMG, Inversão Recuperação e pela CWFP-HR com alternância de fase.

Apesar dos valores de $T_{2}$ obtido estar abaixo do medido pela técnica CPMG, eles estão na 

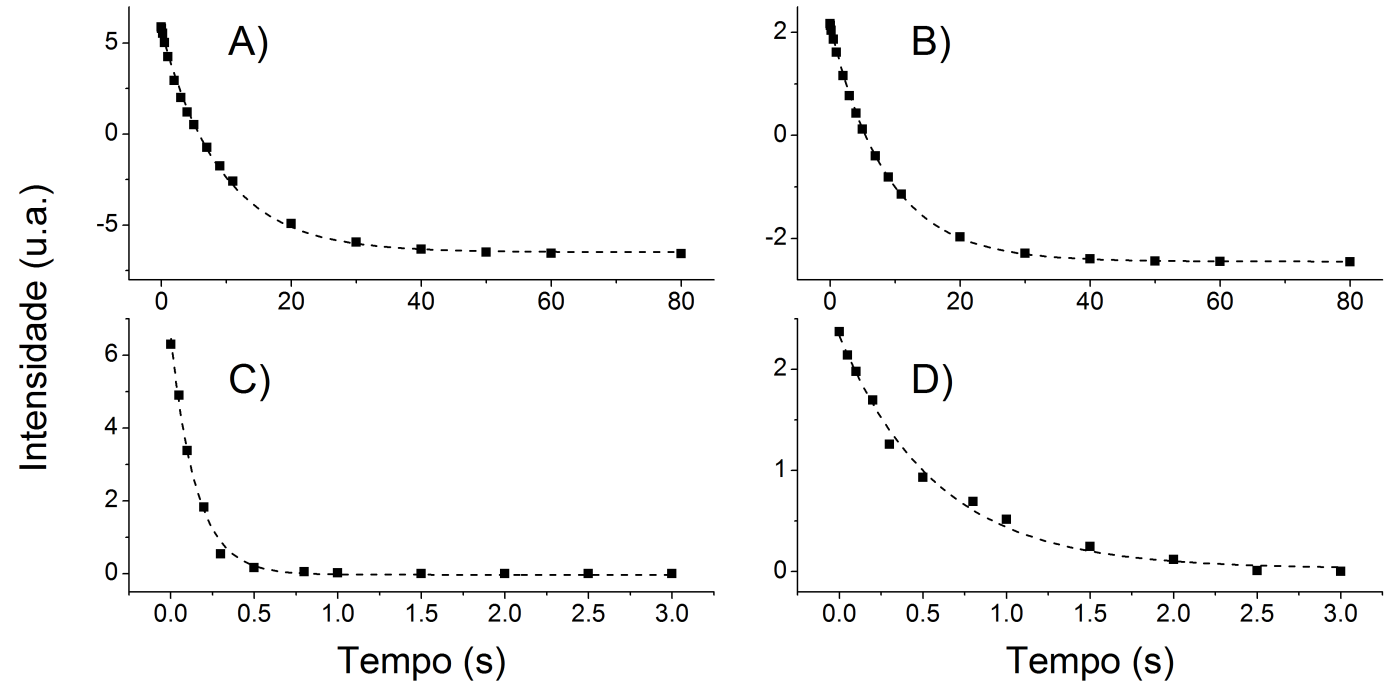

Figura A.2- A) apresenta o sinal adquirido com a sequência Inversão Recuperação para o pico da água e B) para o pico da acetona. C) e D) apresentam respectivamente os sinais adquiridos com a sequência CPMG para o pico da água e da acetona no espectrômetro Bruker $600 \mathrm{MHz}$.

Fonte: Elaborada pelo autor.
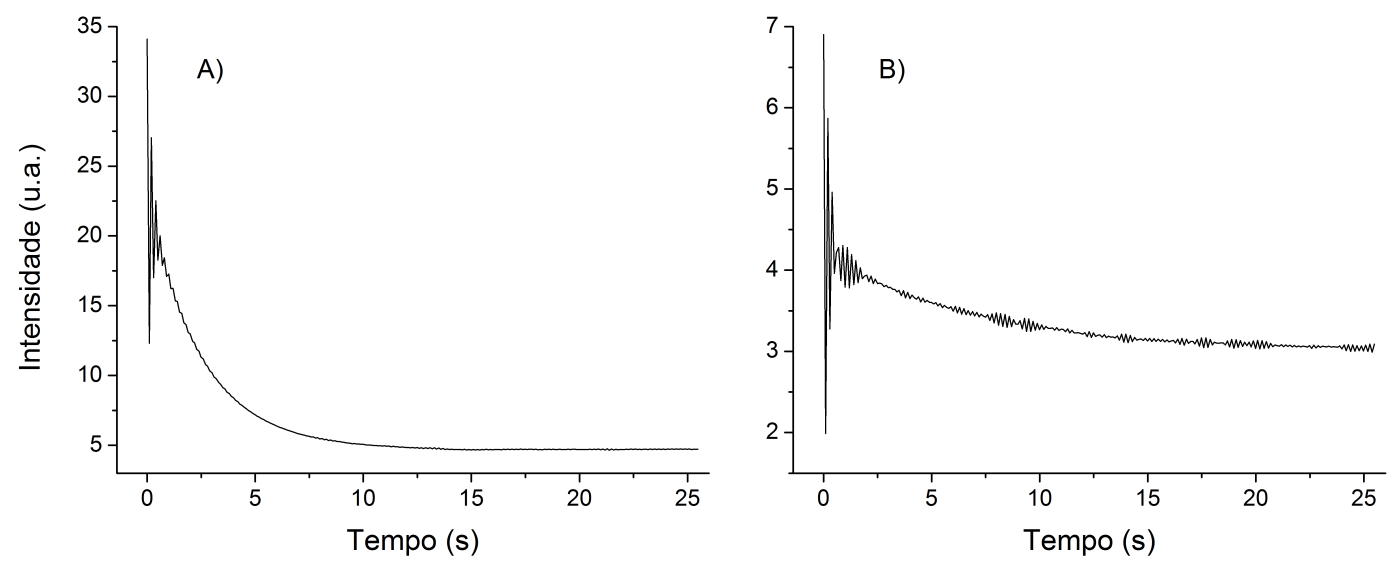

Figura A.3- Decaimento do sinal CWFPx-x em alta resolução $(600 \mathrm{MHz})$ para amostra de $\mathrm{H}_{2} \mathrm{O}$ e acetona até estabilização no estado estacionário do pico da A) água e em B) acetona.

Fonte: Elaborada pelo autor.

Tabela A.1- Comparação tempos de relaxação obtidas com sequências CPMG, Inv. Rec. e CWFP-HR.

\begin{tabular}{c||c|c|c|c}
\hline Seq. Pulso & Inv. Rec. $\left(T_{1}\right)$ & CPMG $\left(T_{2}\right)$ & CWFP-HR $\left(T_{1}\right)$ & CWFP-HR $\left(T_{2}\right)$ \\
\hline \hline $\mathrm{H}_{2} \mathrm{O}$ & $7,7 \mathrm{~s}$ & $2,2 \mathrm{~s}$ & $7,9 \mathrm{~s}$ & $1,4 \mathrm{~s}$ \\
\hline Acetona & $7.4 \mathrm{~s}$ & $7,1 \mathrm{~s}$ & $7,5 \mathrm{~s}$ & $4,5 \mathrm{~s}$ \\
\hline
\end{tabular}

Fonte: Elaborada pelo autor.

mesma ordem de magnitude. No momento estão sendo realizados experimentos para melhorar o calculo de $T_{2}$. Além disso, estamos implementando a SSFP com incremento de fase que possibilitará a determinação de todos os tempos de relaxação num único experimento, sem necessidade de deslocar o offset para cada sinal. 


\section{Apêndice C: Sequências de pulsos SSFP}

Neste apêndice apresentamos as sequências de pulsos SSFP com incremento linear de fase e incremento não-linear de fase: SSFPdx-n4 e SSFPdxdt com $A=\pi / 64$. Os códigos estão na linguagem do software TopSpin 3.2 do espectrômetro Bruker AVANCE III, 600 MHz. Estes códigos são livre para utilização, porém se for utiliza-los para fins acadêmicos ou profissionais, por favor cite esta referência.

A Fig. C.5 apresenta a sequência SSFPdx com incremento linear de fase, com quatro blocos de aquisição do sinal. Os blocos de fase da SSFPdx-n4 utilizam incremento 0, $\pi / 2, \pi$ e $3 \pi / 2$, correspondendo a soma dos perfis A), C), E) e H) da Fig. 5.8. $L_{0}$ é o número de dummy scans antes de cada bloco e $L_{1}$ o número de scans.

A desvantagem de inserir muitos blocos de fases nas SSFPdx é a necessidade de esperar a estabilização de cada estado estacionário para realizar o próximo bloco de aquisição. No caso das sequências SSFPdxdt com incremento não-linear de fase, temos apenas um blocos de dummy scans inicial e as múltiplas aquisições são desnecessárias, simplificando e acelerando os experimentos.

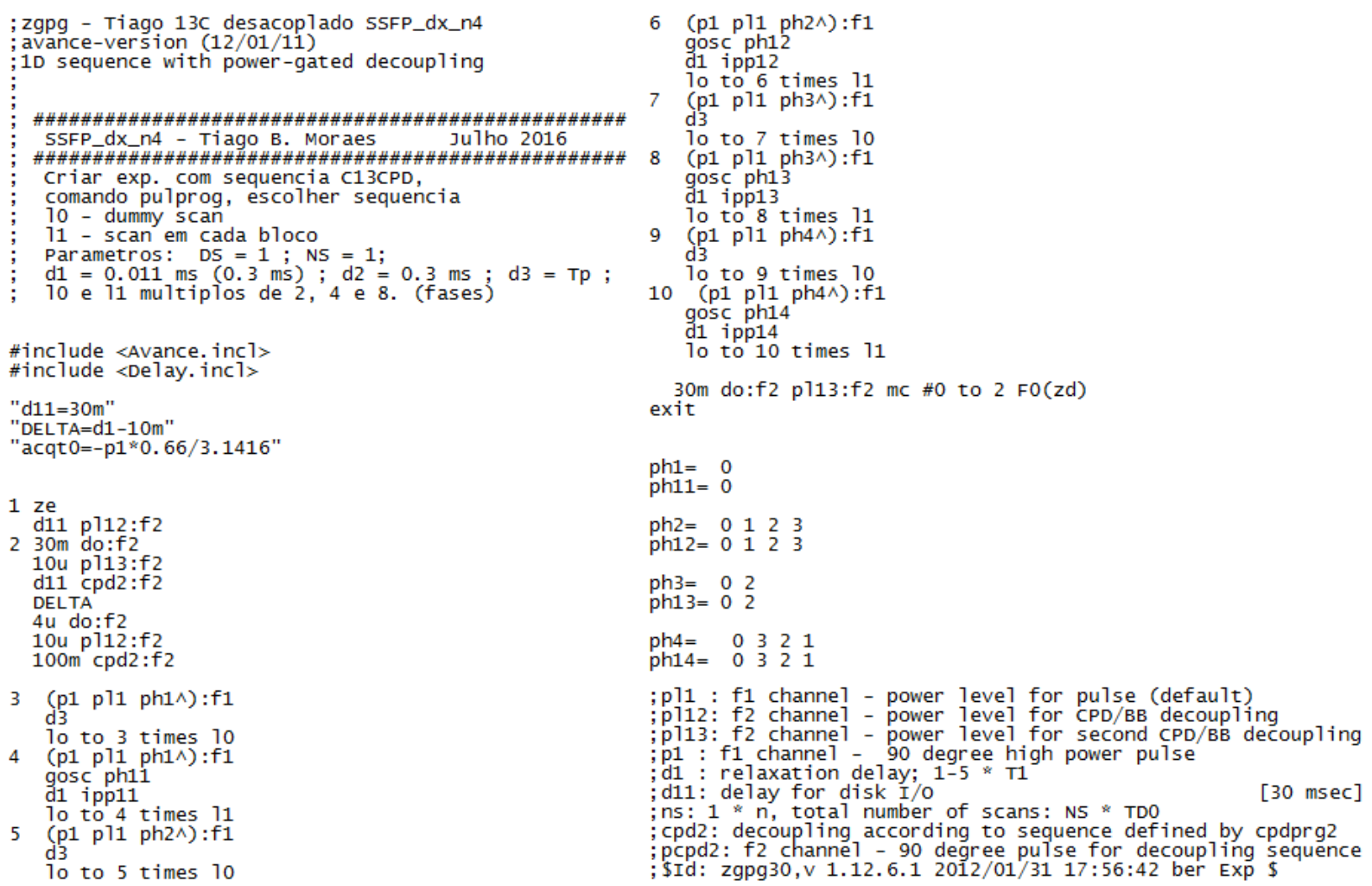

Figura C.5- Sequência de pulsos SSFPdx-n4 com incremento linear de fase, com quatro blocos de aquisição. Fonte: Elaborada pelo autor. 
A Fig. C.6 apresenta a sequência SSFPdxdt com incremento não-linear de fase, utilizando $A=\pi / 64$. Na sequência de pulsos SSFPdxdt o parâmetro $L_{0}$ é o número de dummy scans no transiente inicial da magnetização, que após esse período estabelece o estado estacionário com perfil de excitação modulado no tempo. O número de scans realizados na sequência é definido por $L_{1}$, onde ambos devem ser um múltiplo do divisor do fator $\mathrm{A}, L_{0,1}=n \operatorname{div}(A)$. Garantindo essas condições para $L_{0}$ e $L_{1}$ um perfil de excitação uniforme será obtido. A diferença nas sequências SSFPdxdt com outros valores de A está apenas na tabela de fases.

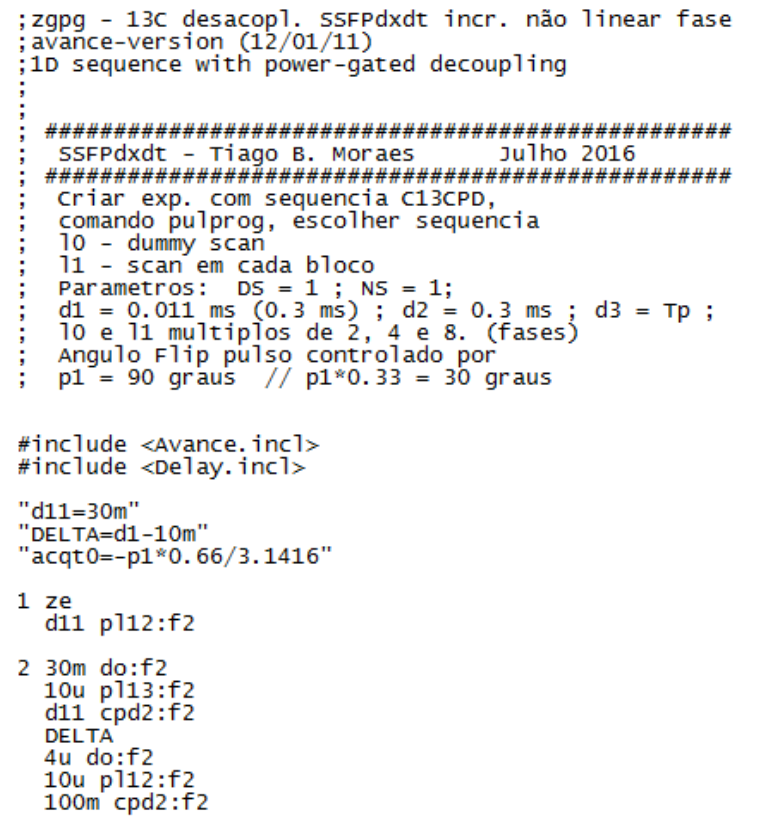

Figura C.6- Sequência de pulsos SSFPdxdt com incremento não linear de fase, utilizando $A=\pi / 64$. Fonte: Elaborada pelo autor.

As demais sequências SSFPdx-n2, SSFPdx-n8, SSFPdxdt são similares as apresentadas acima e podem ser obtidas diretamente com o autor. Dúvidas, sugestões ou versões atualizadas dos códigos podem ser obtidas pelo contato - tiagobuemoraes@gmail.com. 